\title{
AN ANALYSIS OF TRADITIONAL ECOLOGICAL KNOWLEDGE'S STATUS AND ITS CONSERVATION OPTIONS
}

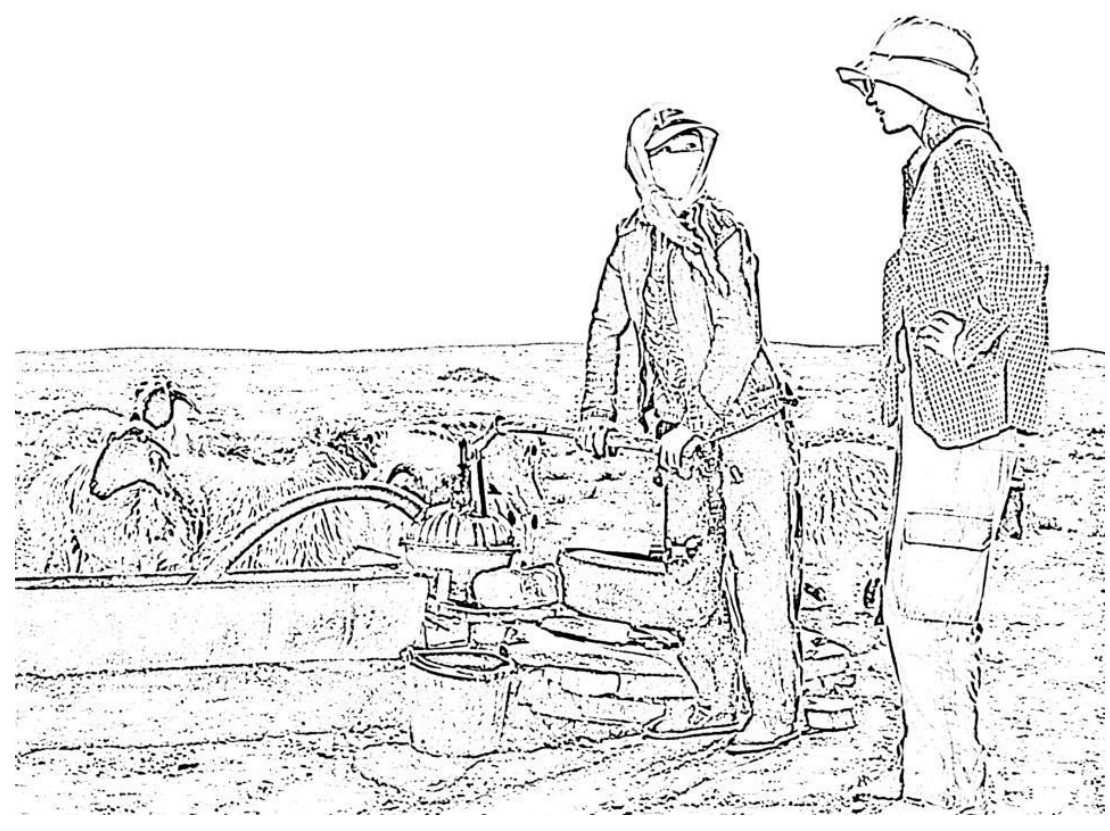

By

\section{Ruifei Tang}

\author{
A thesis
}

submitted to the Victoria University of Wellington in fulfilment of the requirements for the degree of

Doctor of Philosophy

in Environmental Studies

Victoria University of Wellington 

The value of Traditional Ecological Knowledge (TEK) has been recognised and discussed widely in the literature and in public media over the last six decades. Over the same time period, the declining trend in TEK has been frequently reported across multiple case studies in every major region of the globe. As a result, a great number of international agreements (e.g. the Convention on Biological Diversity and the Declaration on the Rights of Indigenous Peoples) have been established in attempt to protect indigenous rights. However, in many cases these agreements have not transformed into concrete TEK conservation actions at the ground level.

Several key literature gaps are identified through this research: the lack of a review of 1) global patterns in the status of TEK; 2) threats to TEK; 3) TEK conservation options; 4) the lack of standard classification systems of TEK threats and conservation actions; 5) the status of TEK and conservation of TEK in Inner Mongolia China is largely unknown to the outside world; and 6) the limited understanding of the role of government policy on TEK trajectories in Inner Mongolia. To develop TEK threat and conservation action typologies is one major target of this research. This is because a standard classification system provides a common language for practitioners to identify problems and solutions, and to communicate across projects. More importantly, it provides a tool for identifying global patterns of TEK and creating a global network and common databases for TEK monitoring, thus to inform conservation actions. However, such a system is missing from the current literature.

Part one of this research applied a mixture of quantitative and qualitative methods, including a global survey $(\mathrm{n}=137)$, its follow-up interviews $(\mathrm{n}=46)$, and a comprehensive literature review $(n=152)$. The qualitative data analysis produced 1 ) a typology of TEK threats; 2) an analysis of the complex causal web among all TEK threats; 3) a typology of conservation actions; 4) the triggers and barriers to TEK conservation success; and 5) three design principles for effective TEK conservation. The quantitative data analysis explored 1) the responses' geographical distribution; 2) the overall trend of TEK change; 
and 3) the significance of different categories of TEK threats and conservation options.

Part two of this research is a case study in Inner Mongolia with two critical outputs: 1) an examination of the impact of Chinese government policies on traditional resource management institutions over time; and 2) an application of the TEK typologies to analyse the current status of TEK and possible interventions in Inner Mongolia. The study applied three qualitative research methods: semi-structured interviews ( $n=91)$, group discussion ( $\mathrm{n}=5$ with 64 participants), and participant observation (eight weeks). The study found that in general government policies (from the1950s - 2007) had negatively affected Mongolian TEK and almost destroyed traditional institutions. However, the new Cooperative Law (2007) may positively impact local TEK by supporting community cooperative organisations and their activities of revitalising traditional herding practices, collective working relationships and traditional leadership. Through applying the typologies to the case study, this research provides a model of how to identify TEK threats and design conservation actions in real-world situations.

Three core messages emerged from this research: 1) dealing with TEK threats and applying TEK conservation have to take a social-ecological system approach, in which social, political, cultural, economic and ecological aspects are all embedded;2) the design and implementation of TEK conservation have to be cross-scale arrangements, in which the institutions from the local level up to the national and international levels are accommodated; and 3) power relations are at the core of achieving sustainable resource management and effective community empowerment. The cooperation between different levels of organisations and among different groups at the same levels requires appropriate power sharing. In a word, TEK is a complex, dynamic and systematic issue, TEK threats take place at multiple levels simultaneously. Therefore, to deal with TEK threats needs a complex, adaptive and systematic approach with holistic worldviews as the intellectual foundation. 


\section{Keywords}

traditional ecological knowledge (TEK), TEK threats, TEK conservation, indigenous peoples, indigenous rights, traditional institutions, institutional linkages, socialecological systems, resource governance decentralisation, power sharing, power devolution, Inner Mongolia, China. 


\section{Acknowledgements}

My greatest THANKS go to my primary supervisor, Dr. Michael Gavin. Your broad knowledge and experience, enthusiasm and wisdom have constantly inspired and guided me to complete this three-year academic journey. I also deeply appreciate your encouragement when I was confused and depressed. I learned enormously from you and enjoyed this learning process. Prof. Phillip Morrison, thank you for taking the role as my secondary supervisor and providing your administrative support to my study since Michael went to work overseas.

From the bottom of my heart, I want to thank all participants in the global survey and in the Inner Mongolia case study. Without your amazing contributions, my research would not have been possible. Thank you all for generously sharing your knowledge, experience and wisdom with me. Special thanks go to Wuritu and Wenduri - my hosts in Bayin Wenduri village in Inner Mongolia. You impressed me with your open arms and sincere generosity.

Dear Dave, Christie, Catherine, Chris Bean, Chris Todd and Joe, thank you so much for taking your time and energy to proofread my thesis and improve the final product of my research.

I am grateful to the sponsors of my PhD study. It was fully funded by the Victoria University PhD Scholarship between 2009 and 2012. This generous funding enabled me to carry out decent research without financial worries. The Royal Society of New Zealand (2010) and the Victoria University Faculty of Science (2011) funded me for presenting my research at multiple international conferences. These conference opportunities were extremely valuable to my research and to my academic merit.

Last and most importantly, I want to thank my husband Erich Eder. You have been my colleague whom I discussed numerous questions and difficulties with, my primary 
proof-reader who read through all chapters not only once (voluntarily or coerced by your wife), my lovely companion for conferences and field work, and my mental supporter when I was depressed and tired. I would also like to thank our baby-to-be. Bo Xun, you have given mum extraordinary energy for completing this thesis! It would be much harder without the expectation of your arrival! 


\section{List of Abbreviations}

CBD: Convention of Biological Diversity

IPR: Intellectual Property Rights

IIED: International Institute of Environment and Development

ILO: International Labour Organisation

IUCN: International Union for Conservation of Nature

IUCN-CMP: International Union for Conservation of Nature - Conservation Measure Partnership

NGO: Non-Governmental Organisation

SES: Social-Ecological System

SPFII: Secretariat of the Permanent Forum on Indigenous Issues

TEK: Traditional Ecological Knowledge

UN: United Nations

UNDP: United Nations Development Programmes

UNCTAD: United Nations Conference on Trade and Development

UNESCO: United Nations Educational, Scientific and Cultural Organisation

UNEP: United Nations Environment Programmes

WIPO: World Intellectual Property Organisation

WHO: World Health Organisation 


\section{List of Figures}

Figure 1.1 An analytical model of the traditional ecological knowledge system 11

Figure 1.2 Applicability of TEK to conservation biology and ecology. 20

Figure 1.3 Decentralisation and devolution in resource and environmental management 38

Figure 1.4 The core subsystems in a framework for analysing SES 39

Figure 2.1 The locations of two target communities in Inner Mongolia, China 60

Figure 2.2 An interview conducted in a household in Bayin gacha 62

Figure 2.3 An interview conducted in the field in Bayin gacha 63

Figure 2.4 A group discussion conducted in a local household 64

Figure 2.5 Participant observation conducted during a community gathering with seven families 65

Figure 3.1 A model of a TEK conservation project 78

Figure 3.2 Map of the survey responses' geographic distribution 81

Figure 3.3 Geographical distribution of survey responses .81

Figure 3.4 Geographical pattern found in the literature .82

Figure 3.5 The overall trend of TEK change - Results of the survey .84

Figure 3.6 The overall trend of TEK change -Results of the literature .85

Figure 3.7 Ratio of direct threats to TEK - Results from the survey 104

Figure 3.8 Ratio of direct threats to TEK - Results from literature review 105

Figure 3.9 Ratio of various categories of underlying threats to TEK

- Results from the survey

Figure 3.10 Ratio of various categories of underlying threats to TEK

- Results from literature 110

Figure 3.11 A model of the causality driving TEK change 113

Figure 3.12 An example of the threat pathways of Loss of pathways of TEK transmission 116

Figure 3.13 An example of the threat pathways of Change of traditional livelihood practices 
Figure 3.14 An example of the threat pathways of Change of traditional religion and beliefs

Figure 3.15 An example of the threat pathways of Change of environment and natural resources 123

Figure 3.16 An example of the threat pathways of Loss of traditional rights 126

Figure 3.17 An example of the threat pathways of Loss of traditional institutions .... 129

Figure 5.1 The pasture in Bayin gacha ………..................................................................... 213

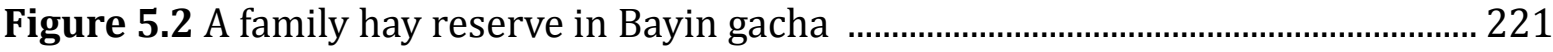

Figure 5.3 The vertical interactions among institutional scales and horizontal collaboration between grassroots level organisations in this case study 245

Figure 5.4 Decentralisation and devolution in resource and environmental management

Figure 6.1 The causal web of TEK threats in Bayin case study 264 


\section{List of Tables}

Table 3.1 Typology of Direct Threats to Traditional Ecological Knowledge .87

Table 3.2 The Typology of Underlying Threats to Traditional Ecological

Knowledge

Table 4.1 Summary of Major International Instruments that Recognise Indigenous

Peoples' Right to Protect their Traditional Knowledge 140

Table 4.2 Typology of TEK Conservation Actions 144

Table 4.3 What has been studied and documented regarding TEK

- Result from the survey

Table 4.4 Commonly Listed TEK Threats and Conservation Options by Geo-regions 180

Table 4.5 Direct TEK Threats and Potential Corresponding Conservation Actions .... 192

Table 4.6 Underlying TEK Threats and Potential Corresponding Conservation

Actions 200

Table 6.1 A Summary of TEK Threats, Conservation Actions and their Links in the Bayin Case Study 270

Table 6.2 The Gap between the Theory and the Practice of Decentralisation 273

Table 6.3 A Summary of TEK Threats, Conservation Actions and Recommendations for Further Conservation Actions in the Bayin Case Study 279 


\section{Preface}

In 1999, when I was working in a snow-mountain area in the southwest of China, I was fascinated by the local indigenous culture and knowledge. Local Tibetan and Naxi herders showed their comprehensive knowledge about their environment and climate, which shocked me somehow as I was there as an "expert" on habitat conservation and environmental education. Through working with local communities, I learned their deep understanding of, connection with, and love for their land. I was also impressed by their enthusiasm in maintaining their traditional culture and practices. Unfortunately, at the time, our organisational focus was habitat conservation for the snub-nosed monkey, and traditional culture conservation was beyond our scope. Even now, I still remember the disappointment in the eyes of Zhongtai (our local guide in mountain research) when we turned down his proposal for a folksong and folk story preservation programme.

After more than a decade of working, studying and researching in the fields of biodiversity conservation, community-based resource management and environmental education, I gradually developed a strong research interest in indigenous knowledge and culture, and realised that I missed a great opportunity in 1999. This regret has motivated my current research. 


\section{Table of Contents}

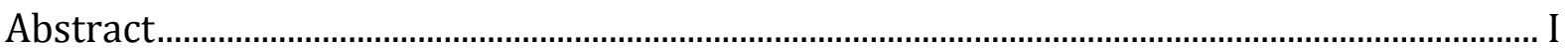

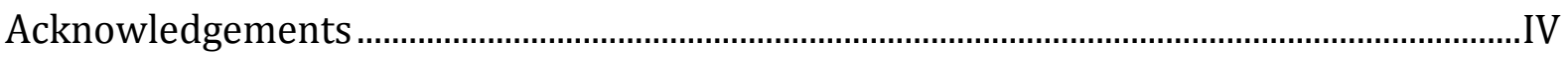

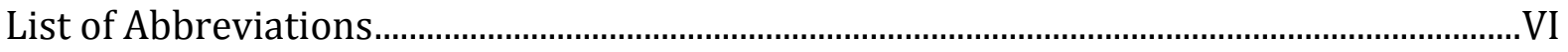

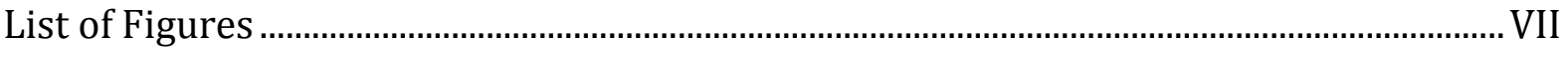

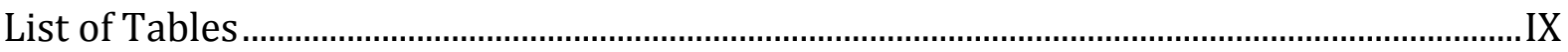

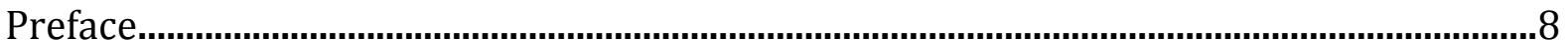

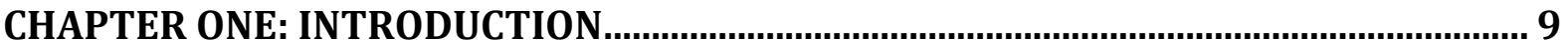

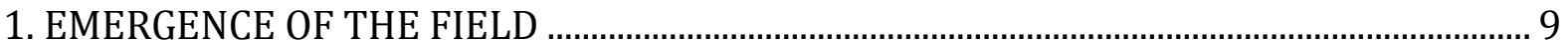

2. THE CONCEPT OF TRADITIONAL ECOLOGICAL KNOWLEDGE ...........................................10

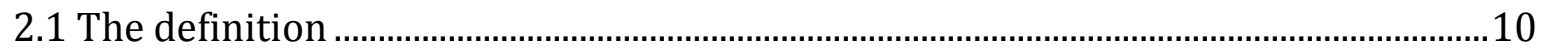

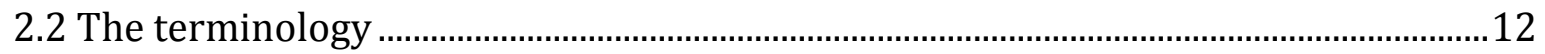

3. THE SIGNIFICANCE OF TRADITIONAL ECOLOGICAL KNOWLEDGE .................................16

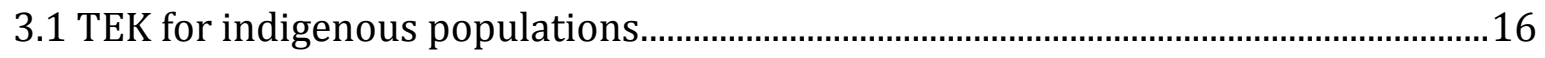

3.1.1 Sustaining indigenous livelihood and well-being ........................................................ 16

3.1.2 Sustaining indigenous cultural identity and significance .........................................16

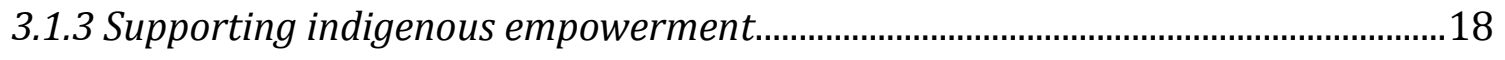

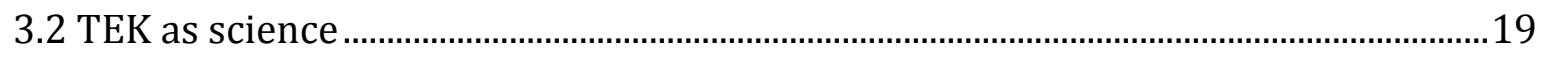

3.3 TEK for environmental conservation and resource management ..............................22

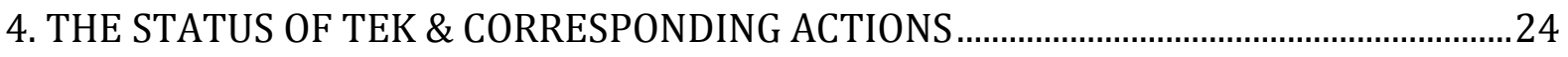

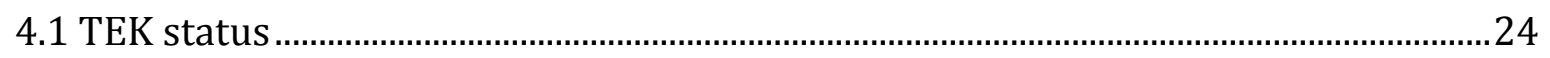

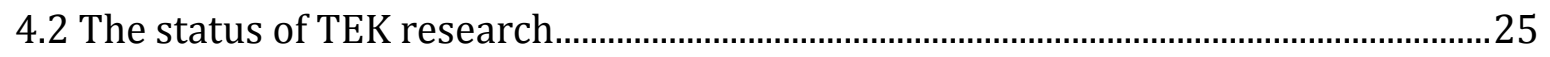

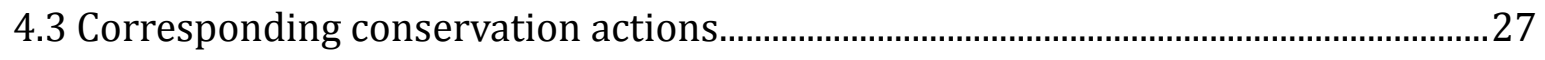

4.4 Controversies regarding TEK and TEK conservation.....................................................28 
4.4.2 Debate over TEK conservation options......................................................................29

4.5 The need for a standard classification system for TEK ….............................................30

4.6 The TEK research gap in Inner Mongolia, China ...................................................................

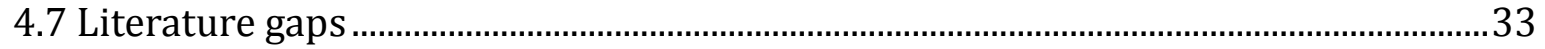

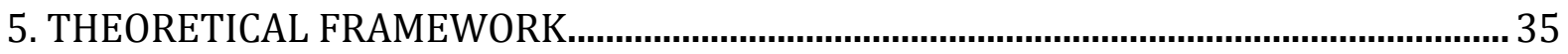

5.1 Managing the commons with good resource governance .................................................35

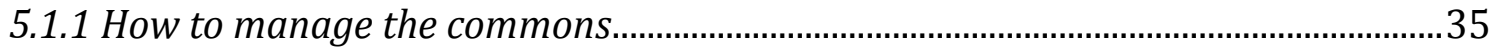

5.1.2 How to achieve good resource governance …...................................................................

5.2 Social-ecological systems and institutional linkages .....................................................

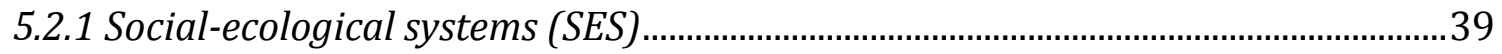

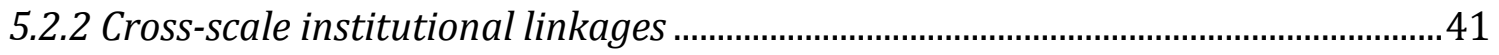

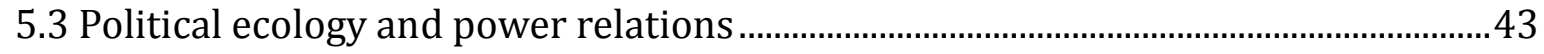

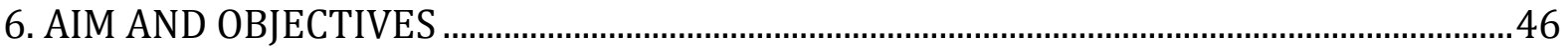

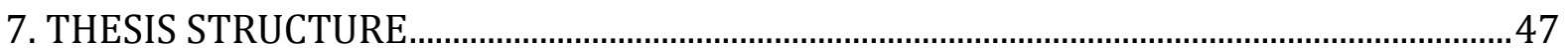

CHAPTER TWO: METHODOLOGY

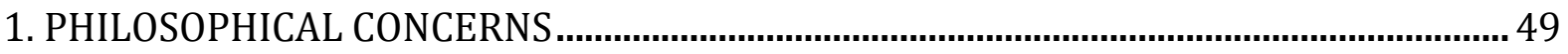

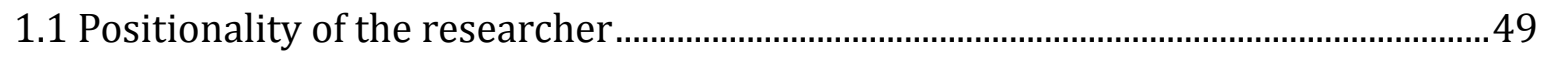

1.2 Cross-cultural research and power dynamics.............................................................. 51

2. ETHICAL CONCERNS AND ETHICAL APPROVAL ……......................................................... 52

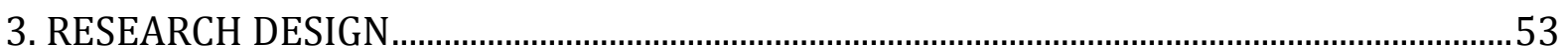

3.1 Part one - Global survey and literature review .............................................................53

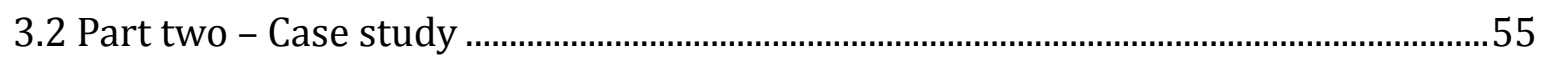

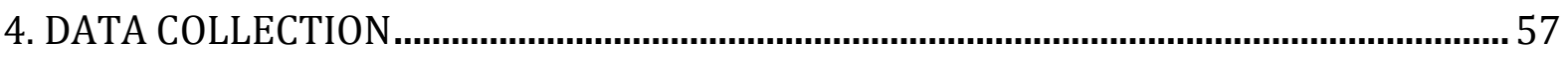

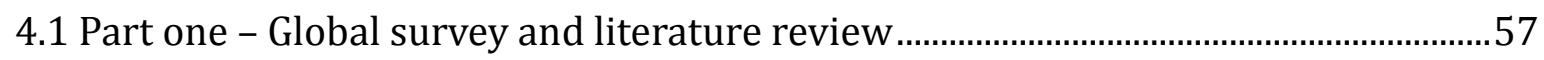




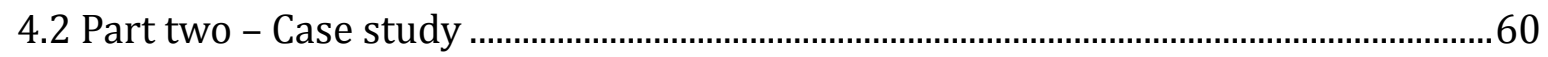

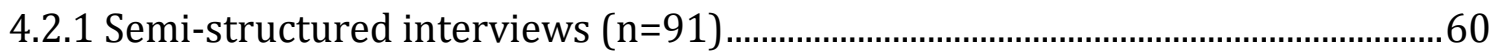

4.2.2 Focus group discussions $(n=5,64$ participants in total) ......................................64

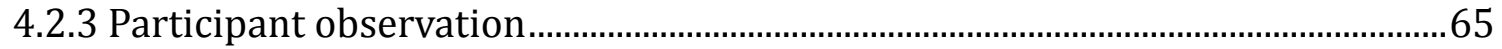

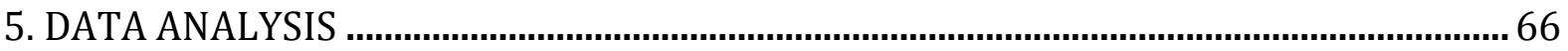

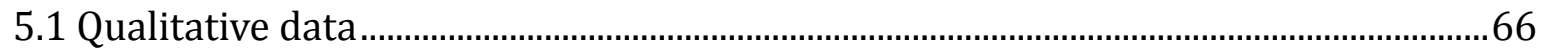

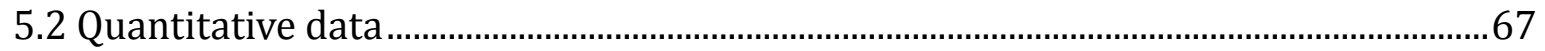

6. LIMITATIONS

CHAPTER THREE: TEK THREATS

1. INTRODUCTION

1.1 Why this study?

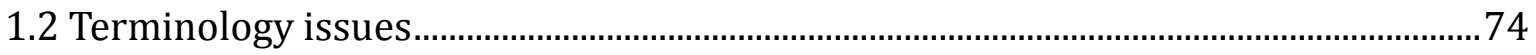

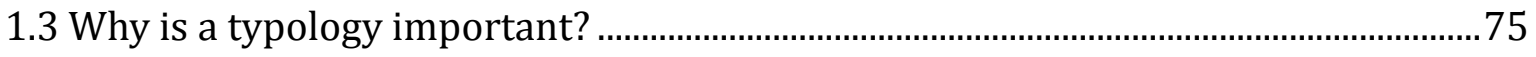

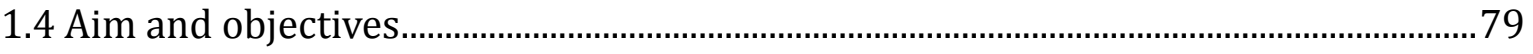

2. RESULTS

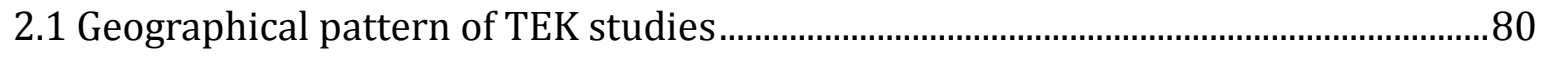

2.1.1 Geographical distribution of survey responses .....................................................80

2.1.2 Geographical pattern found in the literature ......................................................... 82

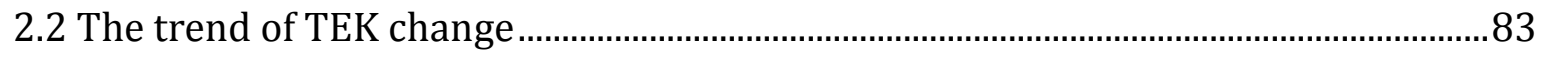

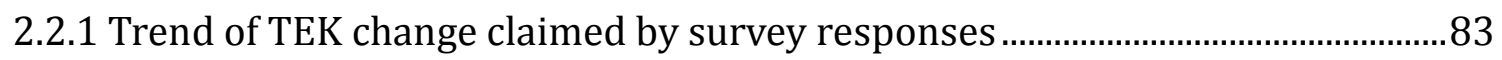

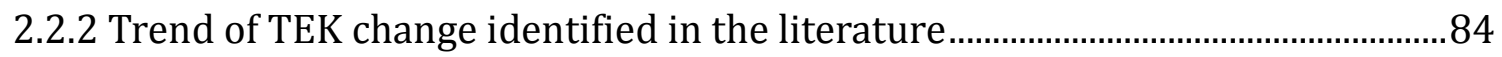

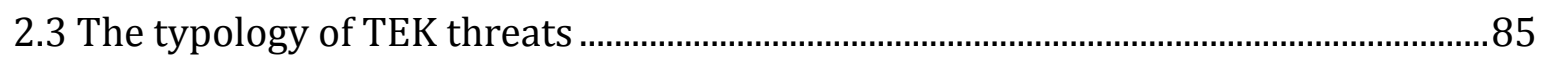

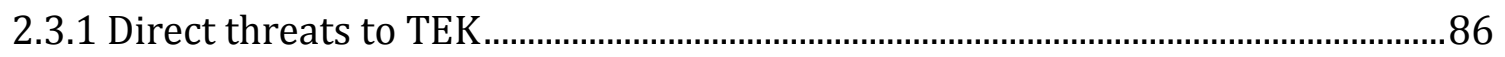

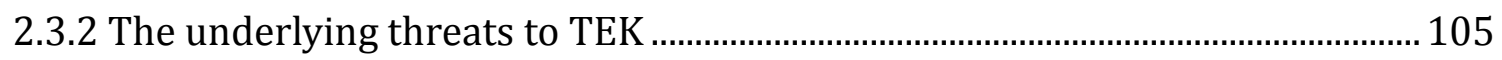

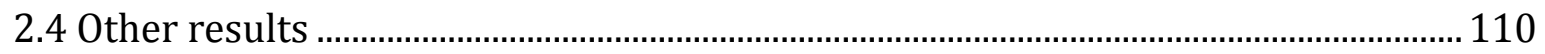


3.1 The complex system of inter-related TEK threats

3.1.1 Threat pathways of loss of pathways of TEK transmission

3.1.2 Threat pathway of change of traditional livelihood practices

3.1.3 Threat pathways of change of traditional religion and beliefs.

3.1.4 Threat pathways of change of environment and natural resources

3.1.5 Threat pathways of loss of traditional rights

3.1.6 Threat pathways of loss of traditional institutions

3.2 Emerging themes from the TEK threat typology. 130

3.3 Power relations and TEK threats 131

3.3 The potential user groups of the typology 134

CHAPTER FOUR: TEK CONSERVATION ACTIONS.

1. INTRODUCTION 138

1.1 The international framework for TEK conservation 138

1.2 Why is this study important? 141

1.3 Aim and objectives 142

2. RESULTS 142

2.1 The Typology of TEK conservation actions... 143

2.2 The conservation options. 148

2.2.1 Action One - Capacity building 148

2.2.2 Action Two - Community-based TEK conservation activities 157

2.2.3 Action Three - Education and awareness. 163

2.2.4 Action Four - Policy and legislative support 168

2.2.5 Action Five - Research and documentation 175

2.3 TEK threats and conservation actions defined by geo-regions 179 
2.4.1 What has been successful and where?

2.4.2 Key reasoning for "successful"/Triggers to success perceived by the survey respondents

2.4.3 Reasoning for failure/Barriers to success perceived by the survey respondents

2.4.4 Reasoning for "hard to say"/other issues. 187

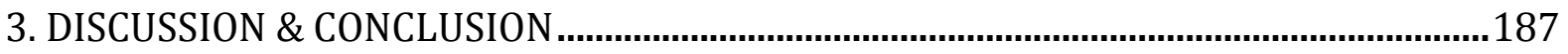

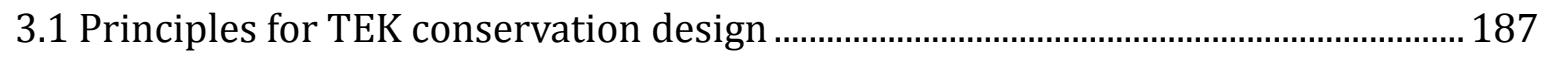

3.2 How to use TEK typology for conservation project design?...................................... 191

3.1.1 Direct threats and potential corresponding actions .............................................. 192

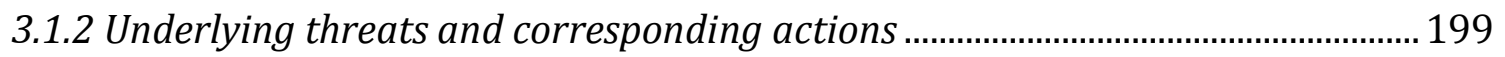

3.3 Limitation \& Recommendations for future research ................................................. 201

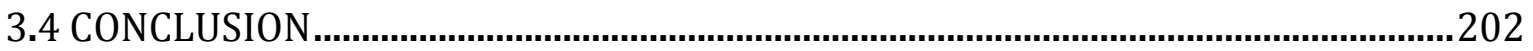

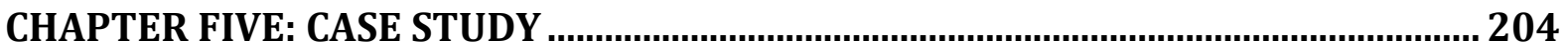

1. INTRODUCTION

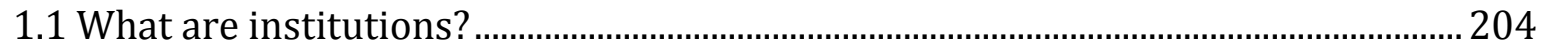

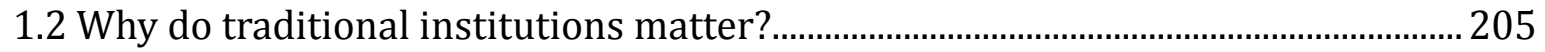

1.3 Why study institutional dynamics in Inner Mongolia, China? ................................... 206

1.4 The Cooperative movement and the Cooperative Organisation Law.......................... 207

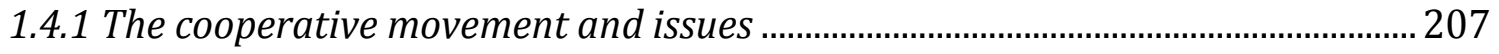

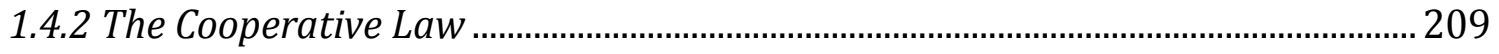

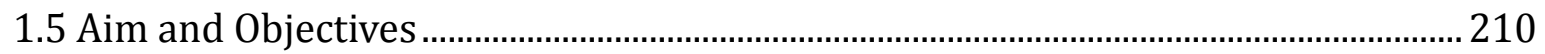

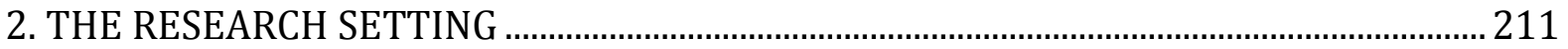

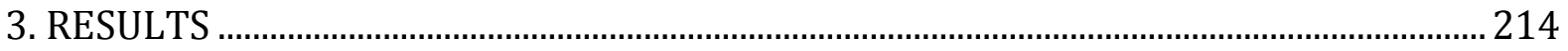


3.1 The governmental policies and their impacts on community institutions and traditional practices

3.1.1 The 1960-1970s, the Grand Commune Era ............................................................ 215

3.1.2 The 1980s-1990s, Settlement and Privatisation Era …....................................... 218

3.1.32000 onward, the Western Development \& Conservation Era................................ 222

3.2 The Cooperative Law implementation at the local level.......................................... 227

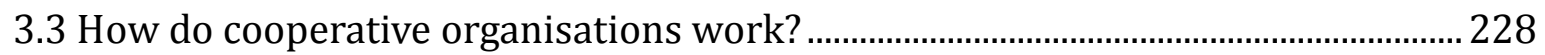

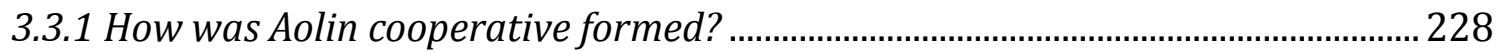

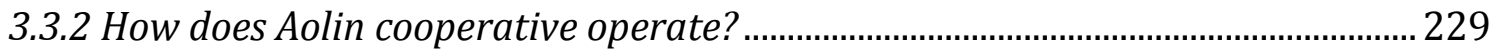

3.3.3 A comparable cooperative from Hu Meng …......................................................... 231

3.4 Perceived benefits and difficulties of cooperative activities ....................................... 234

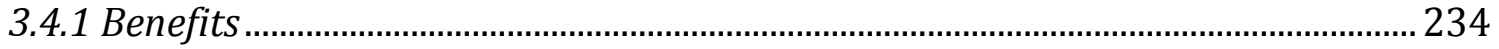

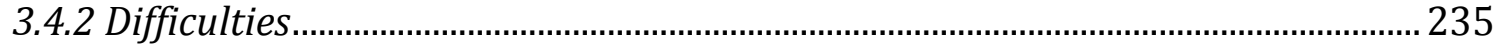

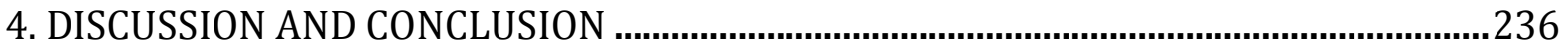

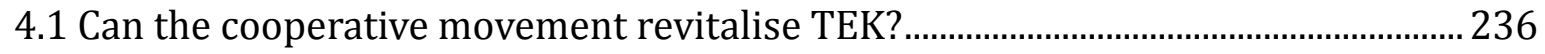

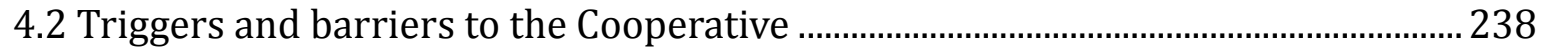

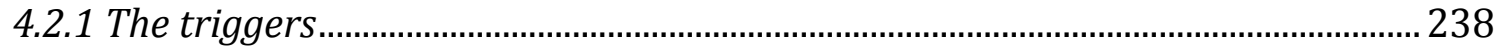

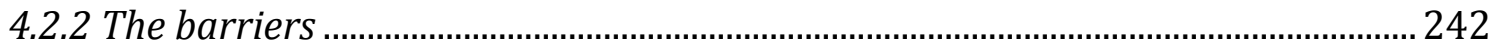

4.3 The institutional linkages and better governance ....................................................... 245

4.4 The limitation of this research \& Possibilities for future research ............................. 248

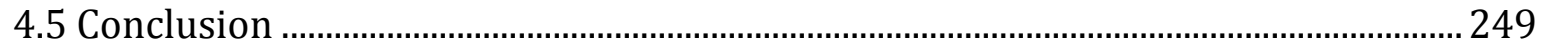

CHAPTER SIX: TYPOLOGY APPLICATION

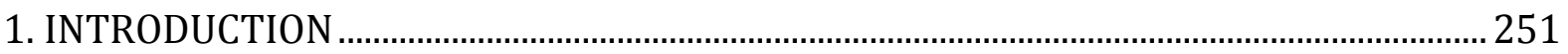

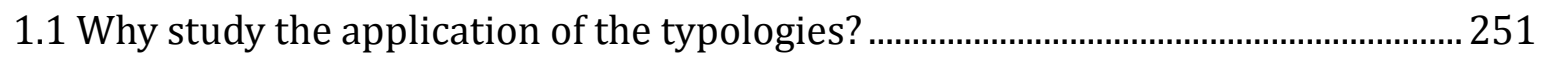

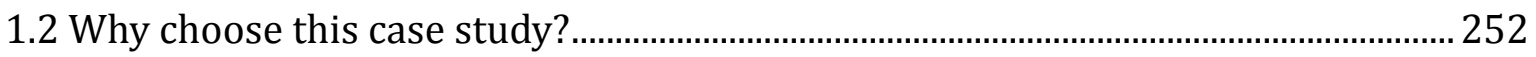


2.1 The change of traditional communal nomadic pastoralism 254

2.1.1 A brief review of the issue 254

2.1.2 TEK threat abalysis 255

2.2 The loss of land or land use caused by mining activities 256

2.2.1 A brief review of the issue 256

2.2.2 TEK threas analysis. 257

2.3 The weak social cohesion when dealing with stress 259

2.3.1 A brief reviw of the issue 259

2.3.2 TEK threat analysis 260

2.4 The grassland degradation and increased cost of production 260

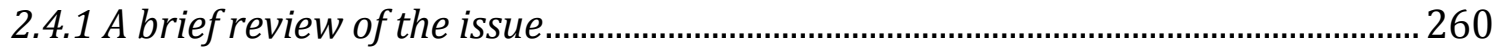

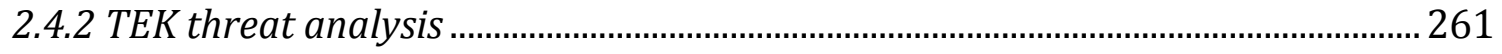

2.5 The absence of younger generations from the community caused by the formal education

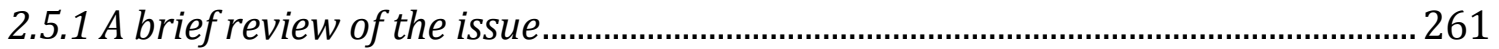

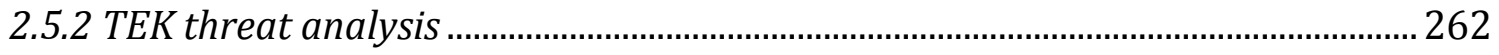

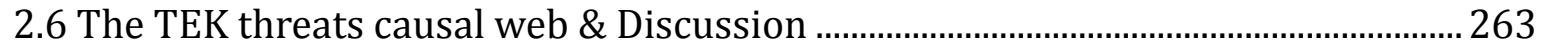

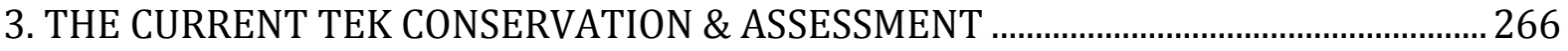

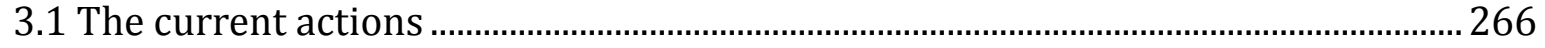

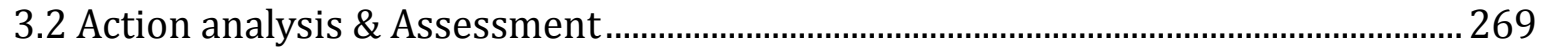

4. RECOMMENDATIONS FOR FURTHER CONSERVATION ACTIONS ……........................... 274

5. CONCLUSION 


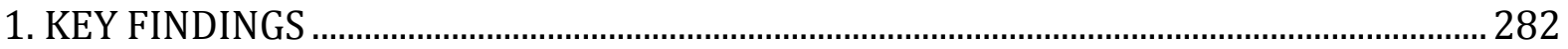

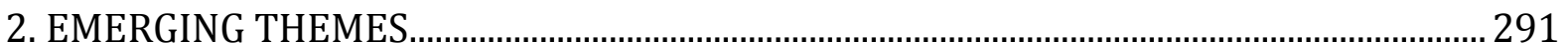

2.1 Applying TEK typologies in a social-ecological system (SES)................................... 291

2.2. Institutional linkages in TEK conservation..................................................................... 292

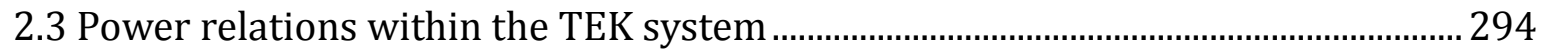

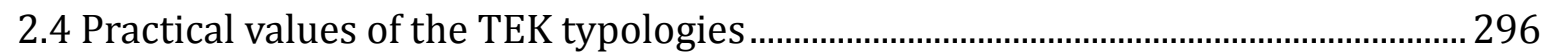

3. LIMITATIONS AND SUGGESTIONS FOR FUTURE RESEARCH ............................................ 297

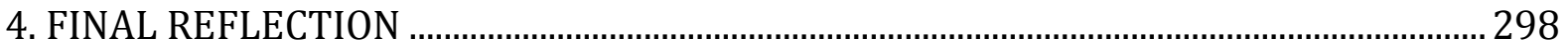

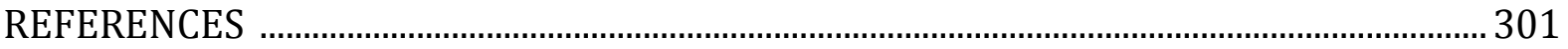

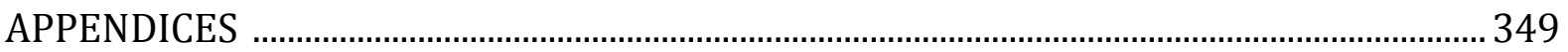




\section{CHAPTER ONE}

\section{INTRODUCTION}

This thesis focuses on exploring patterns in the status of traditional ecological knowledge (TEK), the threats it faces and the options for its conservation. Chapter One will introduce the concept and basic attributes of TEK, the significance of TEK from three perspectives (i.e. TEK for indigenous populations, TEK as science, and TEK for environmental conservation and resource management), the status of TEK research, the theoretical framework of the thesis and its aims, objectives and structure. After describing the research methodology in Chapter Two, the thesis will discuss TEK threats and conservation actions, and present TEK classification systems (TEK typologies) in Chapters Three and Four. A case study examining the impact of government policies on traditional resource institutions in Inner Mongolia will be presented in Chapter Five; and Chapter Six will apply the classification systems of Chapters Three and Four to the Inner Mongolia case study.

\section{EMERGENCE OF THE FIELD}

Traditional ecological knowledge (TEK) has likely existed as long as human society. However, the academic concept and recognition of such knowledge have only been developed in the last five to six decades. The earliest systematic academic studies of TEK were carried out by a group of anthropologists in the 1950s. The research interest in TEK was at first largely held by ethnobiologists and ethnoecologists, who focused on species identification and classification, and indigenous people's understanding of ecological processes and their relationship with the environment (Berkes, 2008). The significance of TEK has been increasingly addressed since the early 1980s, along with the development of biological diversity conservation, sustainable resource management and indigenous rights protection. The value of TEK has also been promoted in various fields, including conservation biology, resource management, environmental studies, development studies and political ecology. 


\section{THE CONCEPT OF TRADITIONAL ECOLOGICAL KNOWLEDGE}

\subsection{The definition}

The academic term of "traditional ecological knowledge" (TEK) was originally defined in the 1970s, and it has only become widely used since the 1980s. There is no universally accepted definition of TEK, but a widely quoted definition was developed by Berkes and his colleagues (2008:7):

[TEK is] a cumulative body of knowledge, practice, and belief, evolving by adaptive processes and handed down through generations by cultural transmissions, about the relationship of living beings (including humans) with one another and with their environment.

This definition covers several key attributes of TEK: 1) TEK consists of biophysical observation, skills and technologies of resource use and management, social relationships and cultural worldviews; 2) it is long standing and transferred from one generation to the next; 3 ) within the processes of transmission, the body of knowledge is usually adapted and amended by new observations and practices; and 4) TEK represents an entity of local knowledge and practices of a specific group, in a specific location (Battiste and Henderson, 2000; Berkes et al., 2000; Fernandez-Gimenez, 2000).

Berkes (2008) points out that TEK is a complex system encompassing knowledge, practice and belief, in which four distinct levels are embedded (see Figure 1.1). 


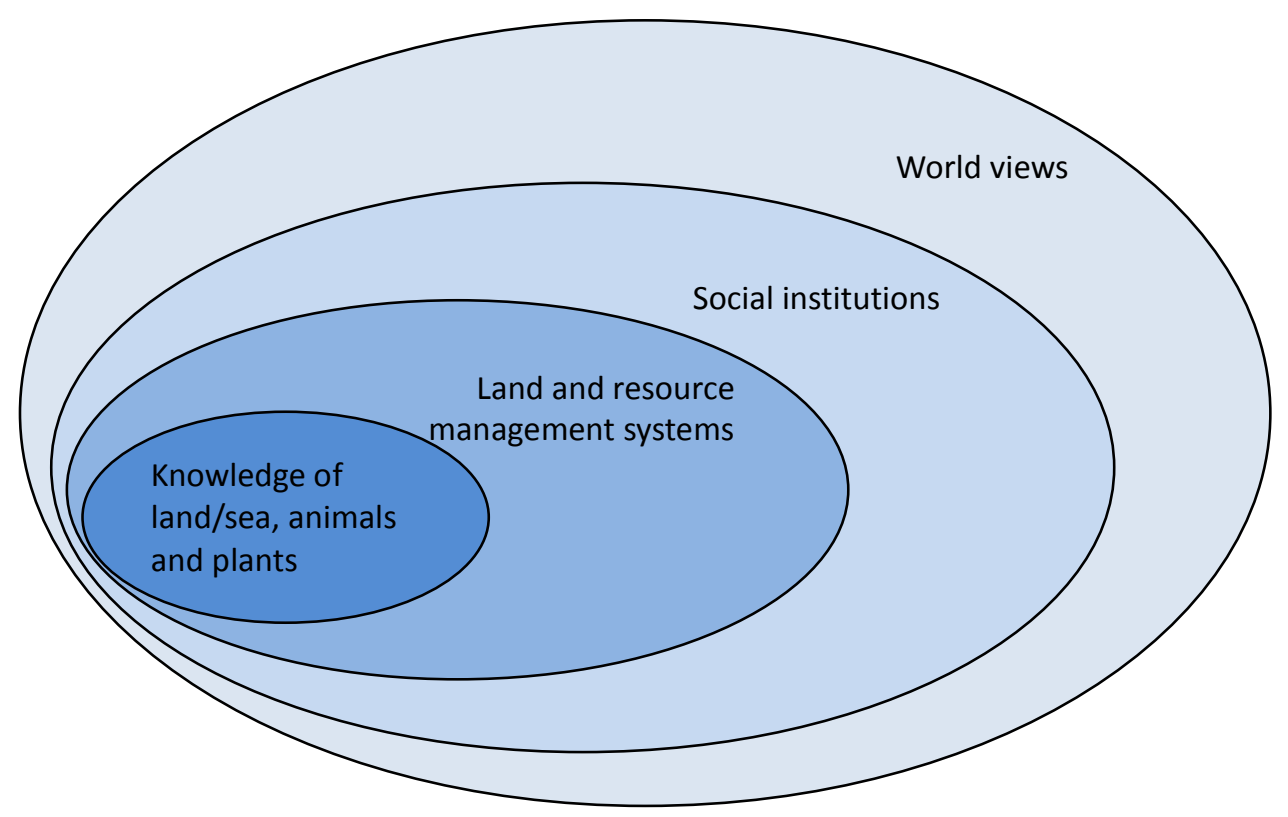

Figure 1.1 An analytical model of the traditional ecological knowledge system. Modified from Berkes, 2008:17

As Figure 1.1 shows, at the central level of the TEK system is the local knowledge of plants, animals, landscapes or seascape and climate. This level of knowledge is often interpreted by Western researchers as species identification and taxonomy, life history, distributions, and behaviours. The second level encompasses a resource use and management system, in which local knowledge is applied, and an appropriate set of practices, tools, and techniques evolves locally (Berkes, 2008). This level of TEK requires the understanding of ecosystem processes and functional ecological relationships. To sustain a traditional resource management system, people have to be socially organised for coordination, cooperation, and rulemaking to provide social restraints and rule enforcement. This requires an adequate social system, which is represented as the third level in the diagram. This social system usually includes a social organisation, a set of rules in use, and codes of social relationships (Berkes, 2008; Houde, 2007). In a wider domain, world views and religious beliefs shape environmental perception and give meaning to observations of the environment, and this forms the fourth level of TEK: cultural expressions and world views (Berkes, 2008). Distinguishing between these 
levels facilitates the understanding of TEK. However, it is important to note that TEK is a holistic, dynamic and adaptive system, in which feedback and interactions among the four levels are constantly operating, and TEK, as a whole, is constantly changing with shifts in local cultural and environmental conditions (Berkes et al., 2000).

The dynamic and adaptive nature of TEK brings up a question to the focus of TEK conservation. How, if at all, can such a dynamic concept be conserved? The TEK conservation I discuss in this thesis contains a broad spectrum, from the actions directly targeting the conservation of single or multiple aspects of TEK (e.g. traditional lifeways programmes; customary education), to the actions targeting underlying causes of TEK degradation and enabling TEK holders (e.g. indigenous capacity building; policy and legislative support). Adequate TEK conservation is not preserving TEK as specimen; rather, it recognises and promotes the dynamic nature of TEK. In other words, it recognises the need of letting TEK holders to decide how TEK should be used and conserved.

In addition to TEK being dynamic and complex, TEK systems are also very diverse both across the globe and even within communities. For example, there are over 6,900 ethnolinguistic groups on the planet, each with its own version of TEK (Maffi, 2001, 2005a). Within each of these groups, diversity also exists among different sectors based on gender, age, social role in the community, etc. (Reyes-Garcia et al., 2007b). In this thesis, because the focus is the global patterns of TEK, the term TEK is used in its general sense that accommodates the common characteristics of all TEK systems. However, this does not undermine the variety and diversity of individual TEK system from different locations and different cultures.

\subsection{The terminology}

TEK is an academic term that was developed on the basis of the Western scientific conception. As some scholars criticise, the phrase "ecological knowledge" may reflect a reductionist approach, which can be viewed as extracting ecological aspects of the 
knowledge and practices from its holistic entity (e.g. Berkes, 2008; Houde, 2007). However, to knowledge holders, usually the indigenous community, TEK is a part of their daily life and overall culture and beliefs, and it can be impossible to separate it from the other components of their traditional system of knowledge, practice, culture and belief (Inglis, 1993). As Berkes (2008) argues, if ecology is narrowly defined as a branch of biology in the sense of Western science ${ }^{1}$, which focuses on interrelationships in the biophysical environment (Friederich, 1958), then traditional ecological knowledge is an oxymoron. However, under a broader definition of ecology, as the study of the relationships of living beings with one another and with their environment (LeviStrauss, 1962), the term becomes tenable.

The phrase "traditional" can also be problematic. In the dictionary sense, "traditional" refers to cultural continuity transmitted in the form of social attitudes, beliefs, principles and conventions of behaviour and practice derived from historical experience (Ellen et al., 2000; Nakashima, 1998). Hunn (1993a:13) further explains “...Traditions are the products of generations of intelligent reflection tested in the rigorous laboratory of survival. That they have endured is proof to their power". However, as Warren (1995:13) points out, "traditional" also signified the 19th century's attitudes of simple, primitive and static. Often, "traditional" is considered as contradictory to change. Another problem with the term "traditional" is the difficulty of defining how much and what kind of change would affect the labelling of a practice as traditional. Some definitions and explanations address the time frame as "historical experience" (Ellen et al., 2000), "products of generations" (Hunn, 1993a) or "handed down through generations" (Berkes, 2008). However, many other definitions of "traditional" do not specify a time frame.

Some scholars, therefore, have developed different terms to illustrate TEK, such as "traditional environmental knowledge" (also TEK), "indigenous knowledge" (IK), "native

\footnotetext{
${ }^{1}$ Western science (or modern science) is a body of empirical, theoretical and practical knowledge about the natural world, produced by researchers making use of scientific methods, which emphasises the observation, explanation, and prediction of real-world phenomena by experiment (Linberg, 2007).
} 
knowledge" (NK) and "traditional knowledge" (TK). However, for each term, problems of accuracy and representativeness also exist. For example, traditional "environmental" knowledge may cover wider domains than merely "ecological" knowledge, but also has the tendency to extract environmental aspects of the knowledge-practice-culture-belief system from its wholeness. "Traditional knowledge", on the other hand, has been criticised (Berkes, 2008) as being too broad and vague, and does not highlight the ecological significance of the traditional knowledge system.

In recent years, "indigenous knowledge" has been increasingly used in academic literature and international policies along with the development of the indigenous rights movement (e.g. Ellen et al., 2000; Sillitoe, 2002; Warren, 1995). The United Nations Environment Programme (UNEP, 2005. http://www.unep.org/ik) defines indigenous knowledge as "the knowledge that an indigenous community accumulates over generations of living in a particular environment. This definition encompasses all forms of knowledge - technologies, know-how skills, practices and beliefs - that enable the community to achieve stable livelihoods in their environment". This definition illustrates the great similarity between indigenous knowledge and traditional ecological knowledge. For example, both concepts address the relationship between a community and its environment, both identify the multiple layers of TEK (i.e. knowledge, skills, management practices and beliefs), and they both highlight the trans-generational character of TEK.

However, as some scholars criticise (e.g. Agrawal, 1995; Berkes, 2008), the term "indigenous" implies a restriction to only the groups that identified as indigenous. According to the conventional definition in anthropology, "indigenous" refers to specific groups of people with ancestral territory, collective cultural configuration, and historical location in relation to the expansion of Europe (Trevor, 1998). The term "indigenous knowledge" then may not represent the recent development and adaptation of the system (Trevor, 1998). As Sundar (2002) points out, indigenous knowledge may eliminate the part of the focal traditional knowledge system that was contributed by non-indigenous groups. The term "native knowledge" faces the same problem as "native" 
is usually a substitution for "indigenous". Despite this academic debate, my research primarily focuses on the knowledge systems held by indigenous populations. This is because indigenous populations are commonly recognised as the main holder of TEK systems (Berkes, 2008; Inglis, 1993), and their societies and TEK are facing constant and various challenges and threats. Therefore, the conservation action typology developed from this research contains a significant number of options concerning the protection of indigenous rights and indigenous empowerment as mechanisms for TEK conservation.

Many indigenous peoples, however, do not like any of these terms. For instance, in Northern Canada, aboriginal people often refer to such knowledge as "knowledge of the land" (Berkes, 2008). In Mongolian culture, the concept of a knowledge system separate from the traditional culture does not exist. The concept closest to TEK in Mongolian culture is the "observation and understanding of nature" (Mu, pers. comm., 2010).

This thesis applies "traditional ecological knowledge" (TEK) as the key term with the definition from Berkes (2008). As Figure 1.1 shows, the concept of TEK encompasses local knowledge, practices, institutions and worldviews as a holistic entity. The study of TEK then does not merely focus on the ecological aspect of a focal knowledge system; rather, it treats TEK as a multi-level, complex system. Another reason for choosing TEK is the existing use of the term. The use of TEK has been officially established through the work of the International Union for Conservation of Nature (IUCN), which named one of its working groups as the Traditional Ecological Knowledge Working Group in 1993 (Williams and Baines, 1993). To date, much research and literature have widely used the concept and the term of TEK. It is practical to use the same term for searching through literature and conducting analysis. However, during the research and literature review process, supplementary key words, including indigenous knowledge, traditional knowledge, and traditional environmental knowledge, were applied to avoid potential gaps (see more discussion in Chapter 2, section 3.1). 


\section{THE SIGNIFICANCE OF TRADITIONAL ECOLOGICAL KNOWLEDGE}

After defining the concept and clarifying the terminology of TEK, it is necessary to explain why and how TEK is important. In other words, why should we study TEK? The next section will analyse the significance of TEK from three different aspects: TEK for indigenous populations, TEK as science and TEK for conservation and resource management.

\subsection{TEK for indigenous populations}

\subsubsection{Sustaining indigenous livelihood and well-being}

TEK is vital for sustaining indigenous livelihood, cultural identity and empowerment. The primary use and importance of TEK relate to its function of sustaining the livelihoods of indigenous populations. While Western societies are increasingly disconnected from nature and cut off their direct reliance on natural resources (e.g. via increasing use of processed goods and services) (Levi-Strauss, 1962; Mazzocchi, 2006), many indigenous communities still rely heavily on natural resources, and their knowledge and skills relating to resource use and management. For example, the knowledge of food sources and medicinal plants still serves a large number of indigenous groups at a substantial level in Asia, Africa, Oceania and South America (Balick and Cox, 1996; Dei et al., 2000; Schultes, 1992). According to the World Health Organisation (2008), about $80 \%$ of the world's people still rely on traditional ethnomedicinal knowledge for their well-being.

\subsubsection{Sustaining indigenous cultural identity and significance}

Conserving and sustaining TEK are also important to maintain indigenous cultural identity and practices. As many scholars point out, there is little or no separation between TEK and other spheres of local culture (e.g. Agrawal, 1995a, 1995b; Natcher et al., 2005, 2007; Simpson, 2005). The knowledge of the biophysical environment is well 
embedded in a wider cultural and social context (Nadasdy, 1999, 2005). For instance, indigenous Tibetan groups on the Qinghai-Tibet plateau consider their hunting activities as spiritual activities rather than pure economic practices for gaining food or cash. Thus, the knowledge of wildlife tracking, local environment and climate as well as hunting techniques has cultural and spiritual value to the local communities (Li, pers. comm. 2010). Similarly, with many North American aboriginal groups, hunting is not merely the mechanical use of their knowledge of animals and environment to obtain food; it is a religious activity (Preston, 2002).

Another example is that almost every indigenous population possesses certain folk songs, dances, ceremonies, sacred objects, narratives and other artifacts. Most of these cultural expressions carry traditional knowledge of the land, and the relationships between their ancestors, themselves and nature (Mulligan, 2003). For example, long song is a very popular kind of folk song among Mongolian groups. As its name indicates, a long song may last for hours. It usually tells historical and current stories of a Mongolian tribe, such as where the tribe originally came from, when and how it moved to the current area, how one of its ancestors fought with wolves, and so on (Zhang, pers. comm. 2010). The ecological aspect of the local knowledge is then transmitted through the song-sing at various cultural activities or social gatherings.

From the above discussions, it is evident that the broader cultural and social aspects are inseparable parts of TEK. To carry on and conserve TEK is vitally important to indigenous groups for maintaining their cultural identity and cultural significance. Therefore, it can be argued that the maintenance of TEK is a fundamental right of indigenous groups. In turn, as many scholars argue, TEK can only be conserved in situ; attempting to abstract TEK from its wider cultural and social system is a typical Western scientific approach, and should be avoided (e.g. Agrawal, 1995a, 1995b; Berkes, 2008). 


\subsubsection{Supporting indigenous empowerment}

Incorporating TEK into conventional conservation and resource management schemes often involves recognising the legitimacy and value of local knowledge, supporting efforts to build local capacity, and sharing power and control of resources with local communities. This empowerment of local people can provide communities with a greater stake in conservation and management initiatives, reducing conflict and improve conservation and management outcomes (Cinner and Aswani 2007; Xu and Melik 2007). Over the last 30 to 40 years, indigenous groups across North America, Latin America, Southeast Asia and Oceania have used TEK as a means to regain control over their culture, and reclaim ownership over their land and resources (Colchester, 1994). For example, aboriginal land-use studies have been carried out in Canada since the 1970s. Many of these studies have been used for aboriginal land claims (Duerden and Kuhn, 1998). Inuit people, for instance, successfully achieved self-governing Inuit territory of Nunavut in 1999, based on a collaborative study of traditional Inuit land use by scientific researchers and local knowledge holders (Berkes, 2008).

Assigning intellectual property rights to TEK and indigenous knowledge of specific resources (e.g. medicinal plants) is another means that can be used to empower indigenous communities. As Posey and Dutfield (1997:15) point out, "indigenous knowledge has been treated as part of the 'public domain', which can be used by any person or corporation as soon as it leaves the community". When researchers publish the results of their works, they may place sensitive indigenous knowledge in the public domain, unwittingly passing it to corporations who can use it for financial gain without any obligation to return benefits to the community (Posey and Dutfield, 1996, 1997). In recent years, some indigenous communities have started to seek legal ownership of, and intellectual property rights in relation to, the use and management of TEK (Dutfield, $2000 \mathrm{a}, 2000 \mathrm{~b})$. In some cases, community-led initiatives have benefited from legal aid and other forms of cooperative support from external organisations (e.g. the World Intellectual Property Organisation and TEK-supporting non-governmental organisations) in their efforts to protect indigenous collective ownership over TEK as an essential indigenous right. 


\subsection{TEK as science}

TEK, as a way of knowing nature and knowledge acquisition, has been compared to Western science. They are both based on accumulative observations of nature (Berkes et al., 2000). A ground-breaking statement made by Levi-Strauss in 1962 points out that traditional knowledge and science are two parallel modes of acquiring knowledge about the universe. "...it [TEK] was no less science and its results no less genuine. They were secured ten thousand years earlier and still remain at the basis of our own civilisation" (Levi-Strauss, 1962: 269). This statement has been considered as ground-breaking because it overcomes Western society's long-standing prejudice against non-Western cultures, especially those which have been erroneously labelled as undeveloped and "primitive". Levi-Strauss prefers the term "prior" rather than "primitive" (Berkes, 2008).

The academic and public interest in TEK arose from discovering its unique value in informing ethnoscience, including ethnobotany (particularly in ethnomedicine), ethnozoology and ethnoecology. For example, the traditional medical knowledge of healers from many indigenous groups has provided important insights into modern pharmacy, such as the development of drugs for cancer, diabetes and obesity (Cox, 2000; Stephenson, 2010). The most recent increase of interest in TEK is largely due to a discovery that such knowledge can contribute to conservation biology (e.g. rare species conservation and protected area management), ecological processes, natural resource management and sustainable development (Alcorn, 1989, 1993; Berkes, 2008; Colding, 1998; Gardgil et al., 1993; Johannes, 1998; Schmink et al., 1992; Tang and Gavin, 2010). It has been shown that traditional knowledge of the environment and local natural history at multiple scales, from species-specific information to details of ecosystem dynamics, often guides scientific research and management decisions, especially in remote areas where Western science-based data are often limited (Drew, 2005; Schultes and Raffauf, 1990). Compared with Western science, TEK has the comparative advantages of understanding and managing the possible "harmonious relationship" between humans and the environment (Berkes, 2008). For example, Silvano and Begossi (2002) compared Piracicabaian Indian views of aquatic tropical structures with 
previous ecological research done by Western scientific researchers. They found that the food web created by indigenous people closely matched the results of scientific research. However, indigenous people also had knowledge of migratory movements of fish that were previously unknown to Western science.

Drew (2005) provides another good example of the application of TEK in conservation biology and ecology (see Figure 1.2 below).

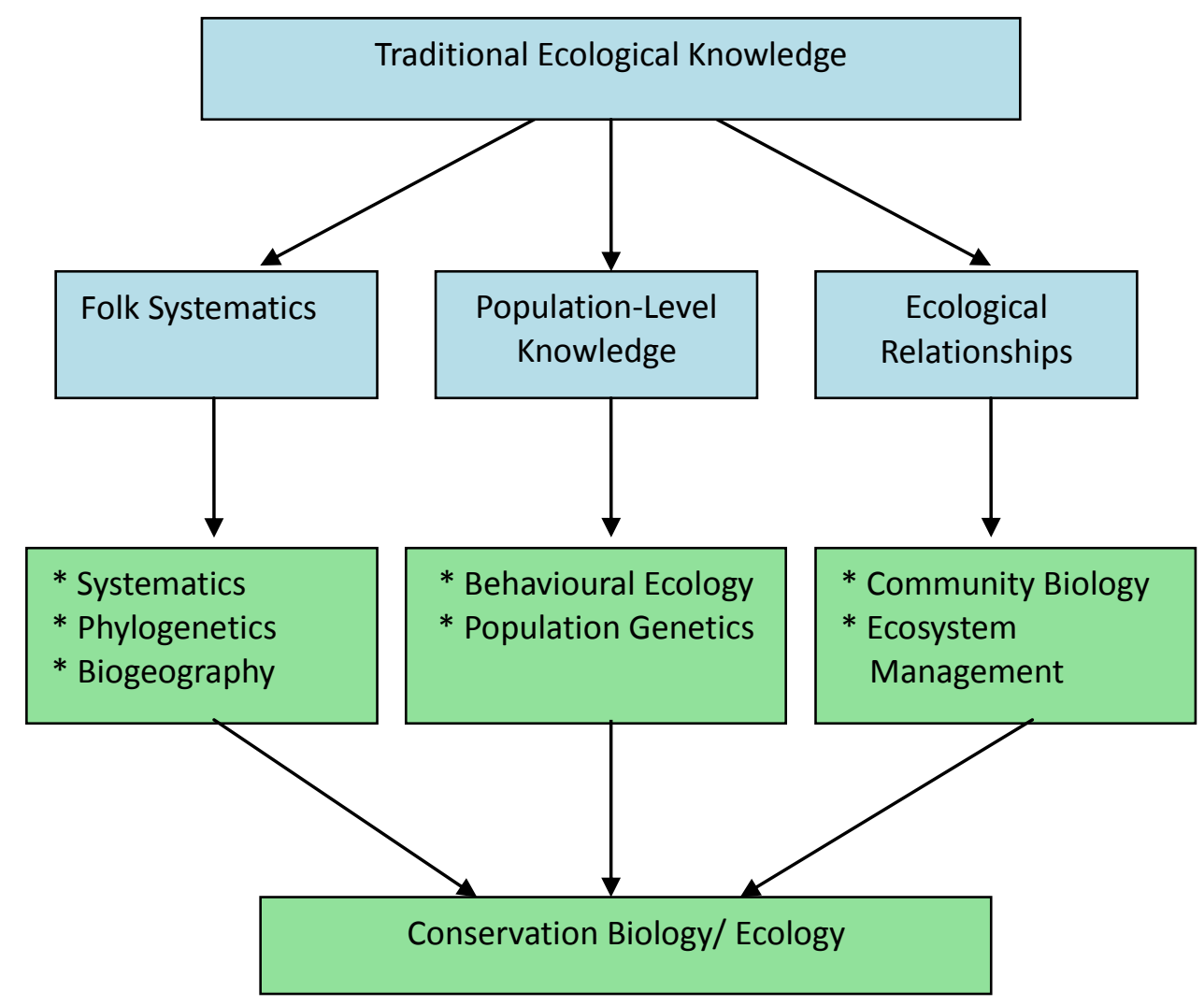

Figure 1.2 Applicability of TEK to conservation biology and ecology.

Modified from Drew, 2005:1289

In this analysis, TEK is divided into three major subcategories that relate to ecological research: folk taxonomy and systematics, population-level knowledge, and ecological relationships. 1) Folk systematics refers to the ways in which different cultures organise their perceptions. Examining these systems of classification is useful in order 
to understand the views of indigenous people about how organisms are grouped and how their culture relates groups of species to the larger world (Berlin, 1992; Boster and Johnson, 1989). 2) Due to their dependence on local resources, indigenous people usually have perceptions of natural fluctuations in population size, habitat specificity, or dietary preferences which often are not yet recorded in scientific literature. Indigenous people are also keenly aware of their impacts on plant and animal populations (Hickey and Johannes, 2002). This population-level knowledge provides insights for behavioural ecology, population genetics, and population-level biology (Drew, 2005). 3) Indigenous people usually possess knowledge about the interactions among species and between species and the local environment, which is gained through multi-generational observations. This community or ecosystem level of knowledge is useful in guiding biologists and ecologists in ecological restoration and management regimes (Nabhan, 2000).

The world far exceeds scientists' capacity for sampling and experimentation, and the knowledge of indigenous people is valuable for providing practical insights and filling the gaps in scientific literature. This is especially true when local records cannot provide enough information in poorly studied areas (Berkes, 2008). Indigenous people, therefore, can sometimes be the only source of local biological and ecological information. Even though there are conceptual and methodological differences between Western science and TEK, these differences should not be overemphasised at the expense of losing the wealth of information that TEK has to offer. Alongside the recognition of the scientific value of TEK, it is also important to respect the fact that TEK is owned by TEK holders. This brings up a critical issue of TEK conservation - the intellectual property rights of TEK. As many scholars argue (e.g. Mathus, 2003; Mugabe, 2000; Stephenson, 2010; Swiderska, 2001, 2004), there is an urgent need to recognise the lawful ownership of traditional resources, knowledge and practices to their owners (usually local indigenous communities), and to apply fair and equitable benefit sharing arising from the use of TEK with the TEK owners (WIPO, 2007, 2011). 


\subsection{TEK for environmental conservation and resource management}

The limited effectiveness of biodiversity conservation and natural resource management has been a major concern in the last few decades. Significant evidence shows that conventional centralised, top-down management regimes have not resulted in sustainable management of natural resources (Ostrom, 2005, 2007a; Velez, 2011; Young, 2006). As many scholars (e.g. Berkes, 2008; Folke et al., 2000; Ostrom, 1990) point out, such regimes have failed to account for the non-equilibrium nature of ecosystems, and often these top-down conservation management systems have failed to apply an adaptive approach to management. Based on the theory of ecological stability and predictability, the conventional approach to conservation and management focuses on achieving controllable yields of natural resources (Carpra, 1996). In turn, it emphasises applying top-down, uniform management rules and practices, and often fails to observe and adapt to environmental feedbacks. On the contrary, TEK is adaptive in nature (Holling et al., 1995). Due to their direct dependence on local ecosystems for livelihood practices, indigenous populations usually "monitor, interpret, and respond to dynamic changes in ecosystems and services that they generate" (Berkes et al., 2000:1252). Thus, TEK often highlights effective and adaptive management options rarely used by conventional conservation.

According to Folke et al. (2000), there are numerous practices that are either largely abandoned or seldom used by conventional management, but found in TEK systems. For instance, management approaches like multi-species management, maintaining ecosystem structure and function, resource rotation and succession management have been abandoned by many governmental management regimes, presumably because of production inefficiency (Alcorn and Toledo, 2000; Altieri, 1994; Folke et al., 2000; Jodha, 1998; Sankhala, 1993; Warren and Pinkston, 1998). A simple example is the practice of resource rotation, which was historically widely employed across the globe. Many herder groups in arid environments traditionally carried out long-distance and seasonal rotations to enable the recovery of grazed rangeland, and to cope with non-equilibrium weather conditions (Niamir-Fuller, 1998). However, the management authorities often have a different approach to rangeland management with a preference for settlement 
and livestock quota reduction (e.g. Dang, 2004; McCarthy et al., 2000). The latter has often been found to fail its goal of conserving rangeland sustainably (e.g. Da, 2006; Niamir-Fuller, 1998).

Moreover, practices including managing landscape patchiness, watershed-based management, managing ecological processes at multiple scales, responding to and managing pulses and surprises, and nurturing sources of ecosystem renewal are commonly found in TEK systems, but often disregarded in conventional management practices (Alcorn and Toledo, 2000; Folke et al., 2000; Gadgil et al., 1998; Holling et al., 1995; Hviding, 2006; Lewis and Ferguson, 1988; Niamir-Fuller, 1998). For example, watershed-based management practices have been used by many indigenous populations in Asia, Africa and Oceania (e.g. Ancient China, Hawaii, Fiji and Solomon Islands) (Ruddle et al., 1992). In ancient Hawaii, for instance, valleys within watersheds were used for integrating farming in combination with agricultural and aquacultural activities (Costa-Pierce, 1987). The ecosystem that the local Hawaiians managed extended from upland forests protected by taboo, to the downstream coral reef and lagoon. Building on this holistic approach to conservation management practice can improve conventional conservation methods, which historically have often adopted a more reductionist focus.

Another significant benefit TEK can offer is that, because TEK comprises a long-term adaptive management framework based on culturally embedded natural resource institutions, the costs of implementing TEK-based conservation can be far less than more conventional approaches (Berkes et al., 2000). By defining local communities as the target of management, the conventional management approach fails to include local incentives towards conservation; instead, it often generates resistance and conflicts, and ultimately results in inefficient actions (Ishigawa, 2006; Tang and Gavin, 2010; Xu and Melick, 2007). On the contrary, employing TEK in conservation and management requires involving and empowering local communities, which can effectively reduce social conflicts and corresponding economic cost (Warren 1991b; Warren et al., 1995). 
The advantages of applying TEK to conservation and resource management have led to international recognition of the need to conserve TEK itself as a vital resource. While debate still rages as to the role local (particularly indigenous) people and their TEK should play in conventional conservation and resource management efforts (e.g. Colchester, 2000; Redford and Sanderson, 2000; Schwartzman et al., 2000), few would deny that consulting local communities regarding their ecological knowledge and possible management options can improve conservation and management outcomes.

In summary, TEK holds important significance for the well-being, cultural identity and empowerment of indigenous peoples. It is also able to inform science and contemporary resource management and conservation practices with practical knowledge and experience that have evolved through adaptation over generations. These values that TEK possesses highlight the necessity of further recognition and immediate conservation of TEK. It is also worth noting that, as discussed earlier, one of the most essential characters of TEK is its continual adaptation to changing environmental and cultural conditions (Berkes, 2008; Nakashima, 1998). Thus any TEK conservation effort will need to recognise and promote the dynamic nature of TEK, and take more holistic and adaptive views to accommodate alternative world views.

\section{THE STATUS OF TEK \& CORRESPONDING ACTIONS}

\subsection{TEK status}

Despite the significance of TEK discussed above, an alarming fact is that a large amount of TEK is disappearing globally. Over the last two to three decades, numerous studies around the world report the loss ${ }^{2}$ of TEK (e.g. Battiste and Henderson, 2000; Florey, 2001; Tang and Gavin, 2010; Zarger and Stepp, 2004; Zent, 2001). For example, within 152 TEK-focused or TEK-related studies that were reviewed in this research (details of

\footnotetext{
2 Please note there is a difference between TEK loss and TEK change. While loss is referring to the declined or degraded status of TEK, change may indicate both decline and increase of TEK. See more discussion in Chapter 3, section 1.2.
} 
this literature review to follow in Chapters 2-4), 89\% of them claimed the loss of TEK from various research locations, including more than 30 countries and regions.

As a critical component of local cultural systems, the loss of TEK has often been observed in association with the loss of cultural diversity. For example, as Sutherland (2003) has noted, the diversity of languages and diversity of local knowledge are both threatened even more than biodiversity worldwide. According to the IUCN (International Union for the Conservation of Nature) Inter-Commission Task Force on Indigenous Peoples (1997, in Dutfield, 2000:5), "Cultures are dying out faster than the peoples associated with them. It has been estimated that half of the world's languages the storehouses of peoples' intellectual heritages and the framework for their unique understandings of life - will disappear within a century." Linguist Michael Krauss (1992) claims that there were more than 6,000 languages spoken at the beginning of the $20^{\text {th }}$ century, and 50\% have disappeared; of those remaining languages, $80 \%$ are spoken only by the elder people. When elders die, as Kirsch (2001) warns, it is inevitable that the distinctive language, cultures and knowledge die with them. This deterioration of language has serious implications for TEK loss, since TEK is mostly passed through oral form within indigenous populations.

\subsection{The status of TEK research}

To date, the vast majority of TEK studies have been location-specific case studies, with few overview studies of the patterns of TEK status or the causes of TEK loss from a wider perspective (Berkes, 2007). The discussion and research about the causes of TEK loss have been widely carried out since the 1970s. Among the 152 studies I reviewed for this thesis with a time range from 1976 to 2010 (see more details in Chapter 2, section 4.1), a great variety of TEK threats have been identified in different studies without a standard classification system. Some scholars emphasise the correlations between language skills, formal education and TEK status. Benz et al. (2000), for instance, claim that developing local language skills in Spanish at the expense of the indigenous language is the main cause of traditional knowledge loss of plant use in the Sierra de 
Manantlan reserve in Mexico. In agreement with Benz et al., Zent (2001) illustrates that the local knowledge of forest trees is negatively correlated with either fluency in Spanish or schooling among Piaroa people in Venezuela. Similarly, Sternberg et al. (2001) find that academic skills, especially math scores, are negatively correlated with the level of traditional knowledge and practical skills in eastern Kenya.

Other scholars analyse the issue from different angles. Godoy et al. (1998) argue that the indigenous Tawahka Indians (in Honduras) who are engaged in the sale of crops or wage labour have less traditional knowledge of wildlife than those who still live on forest products. Harrison (2007b) identifies that the severe reduction in number of wildlife species and range of habitat leads to the loss of TEK about these species and the habitat. Wolff (2001) finds that the movement of indigenous population from rural to urban settings is causing dramatic TEK loss because of their decreased contact with the natural world. These individual case studies from around the world are useful for producing a list of possible TEK threats (e.g. loss of indigenous language, Western-based education systems, market integration, environmental degradation, etc.). However, without a standard means of understanding all possible drivers of TEK loss, and how these different drivers interact with one another, the understanding of TEK status and the potential for dealing with TEK threats through conservation actions at multiple scales will be limited.

As Inglis (1993) points out, TEK has only been studied to a very limited extent at a global level. This trend might be largely caused by the multi-disciplinary nature of TEK research and TEK projects. As Berkes et al. (2000) diagnose, scientists typically examine either ecological systems or social systems, yet the need for an interdisciplinary approach is increasingly obvious. Many ecologists/conservation biologists often lack the skills and sensitivities for social research which are needed for studying TEK; likewise, social researchers often lack the knowledge and skills in ecology, biology and/or resource management to fully appreciate the potential applications of TEK (Ford and Martinez, 2000). Therefore, the emergence of a new field - biocultural conservation, has become inevitable (Maffi, 2001). This combination of biological and social scientific 
disciplines examines the links between biological diversity and cultural diversity, and seeks to develop a common framework for the conservation of both domains. Although biocultural conservationists have attempted to examine the global pattern of cultural diversity in which TEK is embedded as well as the overlap between biological and cultural diversity (Maffi, 2001), the overall status of TEK at the global scale remains largely unknown. This thesis aims to address this literature gap.

\subsection{Corresponding conservation actions}

The recognition of and research interest in TEK conservation have grown significantly in recent years. Numerous international agencies, such as the International Union for Conservation of Nature (IUCN), United Nations Development Programme (UNDP), United Nations Environment Programme (UNEP), United Nations Conference on Trade and Development (UNCTAD), World Intellectual Property Organisation (WIPO), and the World Bank have officially acknowledged the value of TEK, and promoted actions to conserve it (Twarog and Kapoor, 2004). In turn, several international declarations and agreements were produced to identify TEK and indigenous rights and discuss the protection of indigenous rights and traditional practices since the early 1990s. For example, the Convention on Biological Diversity (CBD, 1992) clearly emphasises the need for conserving and promoting wider and more appropriate application of TEK in Article 8(j) and Article 10 (CBD, http://www.cbd.int/convention). The Rio Declaration on Environment and Development (UNEP, 1992) recognises the central importance of indigenous communities in achieving "sustainable development" (see Principle 22 of the Rio Declaration at http://www.unep.org/Documents.Multilingual/Default.asp? Documentid=78). Agenda 21 (1992) addresses the importance of indigenous communities and their knowledge and culture, and the contribution they can make toward protecting biodiversity. It also states that indigenous and local communities should be rewarded for their contribution to biodiversity conservation (UN, 1992 at http://www.un.org/esa/dsd/agenda21). 
However, these international agreements and policies have merely functioned as a guiding tool for national and local level actions. The international agreements do not always lead to real-world action toward TEK conservation. How can these agreements and policies be transformed into action, and how can TEK be conserved at the ground level? These questions remain a major challenge in the field. This has triggered field research to explore TEK conservation options. However, given the short history of TEK research, many of these exercises are considered experimental. For example, within the 152 TEK studies I reviewed in this research, although the vast majority (89\%) of them recognise the necessity of TEK conservation actions, only $11 \%$ of them actually provide or suggest specific mechanisms for taking action.

Even though many TEK holders and practitioners have endeavoured to conserve TEK, the knowledge of available TEK conservation options and the effectiveness of these options are largely unknown. No overall study of TEK conservation actions at the global scale has been published. In this thesis, I aim to identify all possible conservation actions and to examine best practices of TEK conservation through a global survey and a literature review.

\subsection{Controversies regarding TEK and TEK conservation}

\subsubsection{Lack of recognition of TEK by decision makers}

In spite of a wide agreement on TEK's significance, scepticism toward TEK still exists among some academics and most governmental officials. The lack of recognition of TEK by decision makers leads to the difficulty of incorporating TEK into management decision-making processes. As some political ecologists diagnose, this difficulty is rooted in power relationships between decision makers (including governmental officials and Western scientists) and local indigenous experts. These two sets of actors relate to natural resources in different ways, and thus have different political agendas (e.g. Cordell, 1995; Keith and Simon, 1987; Zimmerer and Bassett, 2003). As Keith and Simon (1987: 219) highlight, the issue of authority and legitimacy is the core barrier to the integration between Western science and TEK. They point out that "the behaviour of 
public officials - most notably of wildlife biologists and marine biologists - in conservation debates and disputes often displays a detached arrogance, offensive to northern aboriginal people by its insistence on Western scientific methods as the sole measure of accuracy, and a thinly veiled disdain for traditional knowledge of northern people". This unwillingness to accept and recognise TEK reflects an attitude that TEK is a challenge to the dominant modern reductionist paradigm.

\subsubsection{Debate over TEK conservation options}

Meanwhile, among those who recognise TEK's significance, there is little agreement on how to incorporate TEK into management practices, and how to conserve TEK. For example, some TEK conservation programmes have been initiated using an ex situ approach, which records and stores TEK in external databases (Agrawal, 2002a; Warren, 1995). However, a significant number of researchers critique ex situ conservation as it extracts TEK from its local context (e.g. Brodt, 2001; Zent, 1999). They also argue that TEK is a dynamic system which changes over time, but ex situ conservation does not reflect and accommodate the TEK change (Brodt, 2001; Zent, 1999). Alternatively, critics of ex situ conservation promote in situ uses, such as community-based conservation and co-management, which are based within the local context, involve work with indigenous communities and address TEK holders' needs and preferences (Zent, 1999).

Another major debate exists regarding how to protect indigenous intellectual property rights. Some scholars suggest the use of the existing Intellectual Property Rights (IPRs) system, including patents, plant variety protection and copyrights, to protect TEK (e.g. Mathur, 2003; Mugabe, 2000). The World Intellectual Property Organisation (WIPO, http://www.wipo.int/tk/en0) has also produced numerous reviews of existing intellectual property tools for protecting TEK and sharing its benefits. However, a large number of indigenous communities and scholars argue that IPRs are designed to protect commercial inventions and mostly grant individuals exclusive rights, whereas TEK of communities is first and foremost for subsistence and it is largely held collectively as an ancestral heritage (e.g. Dutfield, 2000; Posey and Dutfield, 1996; Swiderska, 2001). 
Instead, they suggest an alternative strategy - sui generis (literally means "in one's own kind"), which could include database rights, plant breeders' rights and indigenous IPRs (Dutfield, forthcoming; Swiderska, 2006). Yet, such an indigenous IPR system is still under development without practical examples. Other mechanisms, such as educational means (e.g. including TEK in formal educational systems, establishing or revitalising indigenous educational systems) have also been promoted by some scholars (Heckler, 2007; Niroa, 2004; Robert and Angela, 2004).

The debates regarding which conservation mechanisms are most effective commonly exist amongst scholars and practitioners. Perhaps the main cause of these debates stems from the diversity of local contexts and research perspectives. Different locations, local contexts and threats can require different conservation actions to be undertaken accordingly. It is also noticeable that none of the TEK studies I reviewed for this thesis mentioned the assessment of TEK conservation mechanisms. Without proper assessment, it is difficult to know which TEK threats have been addressed and what barriers to TEK conservation exist. This data deficiency, therefore, is likely to obstruct the effectiveness of conservation action.

\subsection{The need for a standard classification system for TEK}

The controversies regarding TEK threats and TEK conservation in the field clearly point to a need to develop standard classification systems to diagnose TEK threats and identify conservation actions. These classification systems can provide an organised structure for TEK holders, practitioners and researchers to access and/or analyse information in an efficient and timely manner (Bruno and Richmond, 2003). As Salafsky et al. (2008), who developed a standard classification system for both threats to biodiversity and possible biodiversity conservation actions, point out, a standard classification system is essential for any scientific discipline.

The need for standard TEK classification systems (TEK typologies) can be argued from at least three angles. First, such systems would assist practitioners (including TEK 
holders and researchers) to identify which types of TEK threats are facing particular indigenous groups, and which actions can be undertaken in response to the threats (Salafsky et al., 2003). Second, the typologies will facilitate cross-project communication by providing a common language for use by practitioners to precisely describe the threats and actions within their own sites (Salafsky et al., 2008). This kind of crossproject learning will encourage the comparative evaluation of conservation practice, and ultimately lead to the development of a more systematic science of TEK conservation (Salafsky et al., 2008, 2009). Third, the typologies will enable decision makers to monitor the frequency and patterns of threats and/or actions across organisational and spatial scales. This will help them to set priorities and allocate resources (TNC, 2006). The next two chapters will discuss the academic significance and practical values of TEK typologies in more detail with examples (Chapter 3, section 1.3 and Chapter 4 , section 1.2).

\subsection{The TEK research gap in Inner Mongolia, China}

To test the TEK threat and conservation typologies created in this thesis, I applied them to a case study focused on changes in TEK (especially regarding traditional institutions) within a focal community in Inner Mongolia. The main reason for choosing this research site is that Inner Mongolia has a high significance in terms of indigenous culture and traditional knowledge in China and in the world. However, the dramatic loss of Mongolian TEK has been reported by many scholars (e.g. Eer, 2007; En, 2005; Fernandez-Gimenez, 2000, 2002; Hasi et al., 2011; Song, 2010; Tang and Gavin, 2010; Williams, 2002; Wu, 2005; Xue, 2009; Ye, 2009). Unfortunately, the recognition of TEK and actions towards the protection of indigenous rights have only been undertaken to a very limited degree (Xu and Melick, 2007; Ye, 2007). This is largely caused by the sociopolitical context in China (Ao, 2005; Wang, 2009). The combination of rapid loss and limited conservation efforts has placed Mongolian TEK in a critically endangered position. 
This research is also inspired by my previous study and work experience in Inner Mongolia (between 2004 and 2007). Between 2006 and 2007, I conducted a case study of utilising TEK into saxoul ${ }^{3}$ conservation in the Alashan region of Inner Mongolia (Tang, 2007). The study finds that Mongolian herders possess detailed knowledge about saxoul ecology and sustainable use of the species and other grassland resources. However, the current top-down resource management regime excludes local communities from decision-making. This has led to the failure of saxoul conservation and increased conflict between the authority and local communities (Tang and Gavin, 2010). This case study presents an example of the failure of top-down, centralised resource governance both ecologically and socially. This trend has been noted by numerous scholars and practitioners across the globe (e.g. Agrawal, 2003; Agrawal and Gibson, 1999; Anderies et al., 2004; Berkes, 2002, 2007, 2010; Ostrom, 1999, 2009).

In turn, resource governance decentralisation has been promoted by a significant number of scholars in recent years (e.g. Agrawal, 2002a, 2002b; Berkes, 2010; Ostrom 1990). Cross-scale governance has been recognised as an appropriate regime for sustainable resource management (Pahl-Wostl, 2009). This is because managing complex social-ecological resource systems requires a full range of knowledge and skills that is difficult for any single group or agency to possess (Berkes, 2010; Liu et al., 2007). Therefore, sustainable resource governance needs the inclusion of multiple stakeholders across different institutional and spatial scales. Nonetheless, the study of the possibilities of cross-scale governance, especially the role of community institutions in resource management in China is largely missing in the literature. For example, there is no documentation of the impacts of governmental policies on TEK and indigenous Mongolian populations in the literature. In fact, there is only a limited number of publications that discuss governmental policy effects on indigenous people in China in general (e.g. Da, 2008; Da and Zheng, 2006; Wang, 2007, 2009). This research will fill this literature gap.

\footnotetext{
${ }^{3}$ Saxoul (Haloxylon ammodendron)is a keystone tree species in the Gobi desert ecosystem.
} 
A new trend, called the cooperative movement, has been noted since the late 1990s in Inner Mongolia and China more broadly. The movement is characterised by community initiatives for developing economic organisations that aim to strengthen community cohesion and its competitiveness in the market activities. In Inner Mongolia, starting in the 1990s, an unknown number of communities started to form organisations in which they can carry out traditional practices, such as seasonal herding rotations and sharing production equipment. These organisations mainly focus on improving production and trading activities at the community level, as well as preserving traditional cultural heritage (Ba, 2010). Members of such organisations take collective actions in market exchanges, and share environmental and market risks.

With the goal of bridging the economic and social disparity between urban and rural populations, the Chinese state government enacted the China Farmers' Professional Cooperative Organisation Law (Cooperative Law) in 2007. The law recognises the legal title of farmers and herders' organisations, and provides financial, taxation and registration support to the development of cooperatives organisations (see more details about the Law in Chapter 5, section 1.4.2).

Although TEK conservation was not a goal of the legislation, the law has opened up the possibility of revitalising traditional practices and institutions in Inner Mongolia. My case study (presented in Chapter 5) will examine this possibility and fill a significant gap by noting how cross-scale institutional linkages impact traditional resource management institutions in China.

\subsection{Literature gaps}

From the above discussion, several literature gaps have emerged:

1. To date, most of the TEK studies are location-specific case studies. There is a limited number of overview studies that focus on the global patterns in the status of TEK (section 4.2). 
2. Research on the causes of TEK loss on a case-by-case basis has been carried out for decades, but what is now needed is a comprehensive global review of TEK threats (section 4.2).

3. While the urgency of TEK conservation has been highlighted through multiple international declarations and agreements, there is no systematic study or comprehensive review of available TEK conservation options (section 4.3).

4. A standard classification system can provide a common language and standard for scientists and practitioners to apply in order to communicate, compare and cooperate. However, such as a system for TEK threats and conservation actions has not been developed so far (section 4.2, $4.3 \& 4.5$ ).

5. The TEK and traditional culture of Inner Mongolia hold great significance for both China and the world more broadly. However, studies of TEK status and TEK conservation in Inner Mongolia have only been carried out at a very limited level (section 4.6).

6. There is very limited understanding of how government policy and legislation affect TEK and TEK conservation at the community level, particularly in relation to traditional resource management institutions in Inner Mongolia, China (section 4.6).

The first part of this thesis seeks to remedy the first four gaps in the literature identified above. I conducted a global survey and literature review to explore global patterns in TEK status, causes of TEK change and TEK conservation actions. Based on these findings, I developed TEK typologies to enable links to be made between threats and conservation actions.

To address the last two literature gaps identified above, the second part of this thesis provides an in-depth case study on the causes of TEK change in Inner Mongolia and possibilities for its revitalisation given the complicated socio-political conditions in 
China. I also explore the possibilities for community-based resource management through revitalising community-level institutions.

\section{THEORETICAL FRAMEWORK}

Much of the above discussion has provided a general outline of TEK and TEK studies, as well as research approaches of a classification of global TEK threats and conservation, and the case study in Inner Mongolia. This section focuses on how theories from different fields have been applied to inform the analysis of TEK issues and the development of a TEK classification system throughout this thesis. The key theories which form a framework for this thesis are pulled from the commons literature, socialecological systems theory (in particular, the importance of institutional linkages), and political ecology with an emphasis on power relations and power sharing and their effects on resource management.

\subsection{Managing the commons with good resource governance}

\subsubsection{How to manage the commons}

How to effectively and sustainably manage common resources has always been a central challenge for human society (Ostrom, 2009). Despite the long history of traditional communal management regimes of commons resources in many locations, the modern commons management regime is based on the theory of the Tragedy of the Commons developed by Hardin (1968). This regime is characterised by state-controlled arrangements, and is referred to as common resource centralisation. The underlying assumptions of this approach include: 1) resource users are incapable of overcoming their temptation to harvest excessively from a common resource; 2) designing rules to change the incentives of participants is a relatively simple analytical task; and 3) an organisation itself requires central direction (Hardin and Baden, 1977). Under this approach, policy analysts and decision makers consider common resources (e.g. fisheries, forests, pasture lands and water resources) as relatively homogeneous 
systems that can be treated with uniform rules (Sherman and Laughlin, 1992). The management of common resources has been treated as a scientific and technical matter in recent decades, and central governments have held the responsibility for applying technical expertise to design appropriate rules for governing and managing common resources (Bocking, 2004; Ostrom, 2007a). The danger of this approach lies in its management of complex social-ecological systems as a simplified, homogeneous system.

Since the 1980s, centralised environmental and resource governance has been widely questioned and challenged. Much field research has found that national governmental agencies have been notably unsuccessful in their efforts to design effective and uniform rules for regulating important common resources across a broad domain (Ascher, 1999). As Berkes (2010: 489) points out, "By the late 1980s, there was a general disillusionment of stakeholders, conservation and development agencies, and academics in the ability of centralised governments to plan, administer and implement conservation and development".

The failures of centralised regimes have forced reconsideration of who should be involved in decision-making to ensure better outcomes from commons management. The rights of resource users to be involved in decision making, and the significant role of community institutions in sustainable resource management have been increasingly recognised by many scholars and practitioners (e.g. Agrawal, 2002a; Berkes, 2002, 2010; Leach et al., 1999; Ostrom, 2005, 2007a, 2009). Many scholars have also examined the capacity of communities and other small-scale social organisations to self-govern and manage resources (e.g. Agrawal, 2003; Agrawal and Ostrom, 2001; Ostrom, 1990; Ostrom and Jassen, 2004).

As a result, an environmental governance reform has taken place starting in the 1990s. The reform has emphasised a shift from the conventional top-down governance towards co-management, community-based resource management and self-governing resource management (Brondizio et al., 2009; Ostrom, 2009). The logic behind this new thinking is to bridge the gap between governments and the governed and allow the people whose 
livelihood and well-being are affected by the decisions to participate in making those decisions (Berkes, 2010). In particular, as Agrawal and Gibson (1999) argue, more community-based management regimes allow communities to contribute their traditional knowledge of the local environment to resource management practices. Often, within these community-based practices, communities' traditional rights over the land and resources are recognised and protected. Over the last 20 years, almost all the national governments across the world, both in developing and industrialised countries, have undergone a decentralisation process, although to varying degrees (BorriniFeyerabend et al., 2004; Brunner et al., 2005; Wilson et al., 2003). For example, many national governments in developing countries have changed their renewable resource policies in favour of establishing and strengthening partnerships with local-level organisations (Lemos and Agrawal, 2006).

\subsubsection{How to achieve good resource governance}

Within resource governance reform, effective resource user participation and problem solving at more local levels of an organisation, as well as the subsidiarity principle ${ }^{4}$ were considered as essential components of "good governance" (Kooman, 2003). These principles have been advocated by international policies, such as Agenda 21 and the Rio Declaration (UNCED, 1992). While resource governance decentralisation has become "the new fashion in our time" (Berkes, 2010), some scholars have carried out further investigation and analysis, and identified two phases (or two types) of this process: decentralisation and devolution (Berkes, 2010). As Brugere (2006) clarifies, there is an important distinction between decentralisation and devolution. While decentralisation illustrates the transfer of rights and responsibilities from central to branches of the same government ministry; devolution represents the transfer of rights and responsibilities to local groups, organisations and local level government decisionmaking power (see Figure 1.3).

\footnotetext{
${ }^{4}$ The principle emphasises that issues regarding resource management should be handled by the smallest, lowest, or least centralised authority that are capable of addressing the issues effectively.
} 


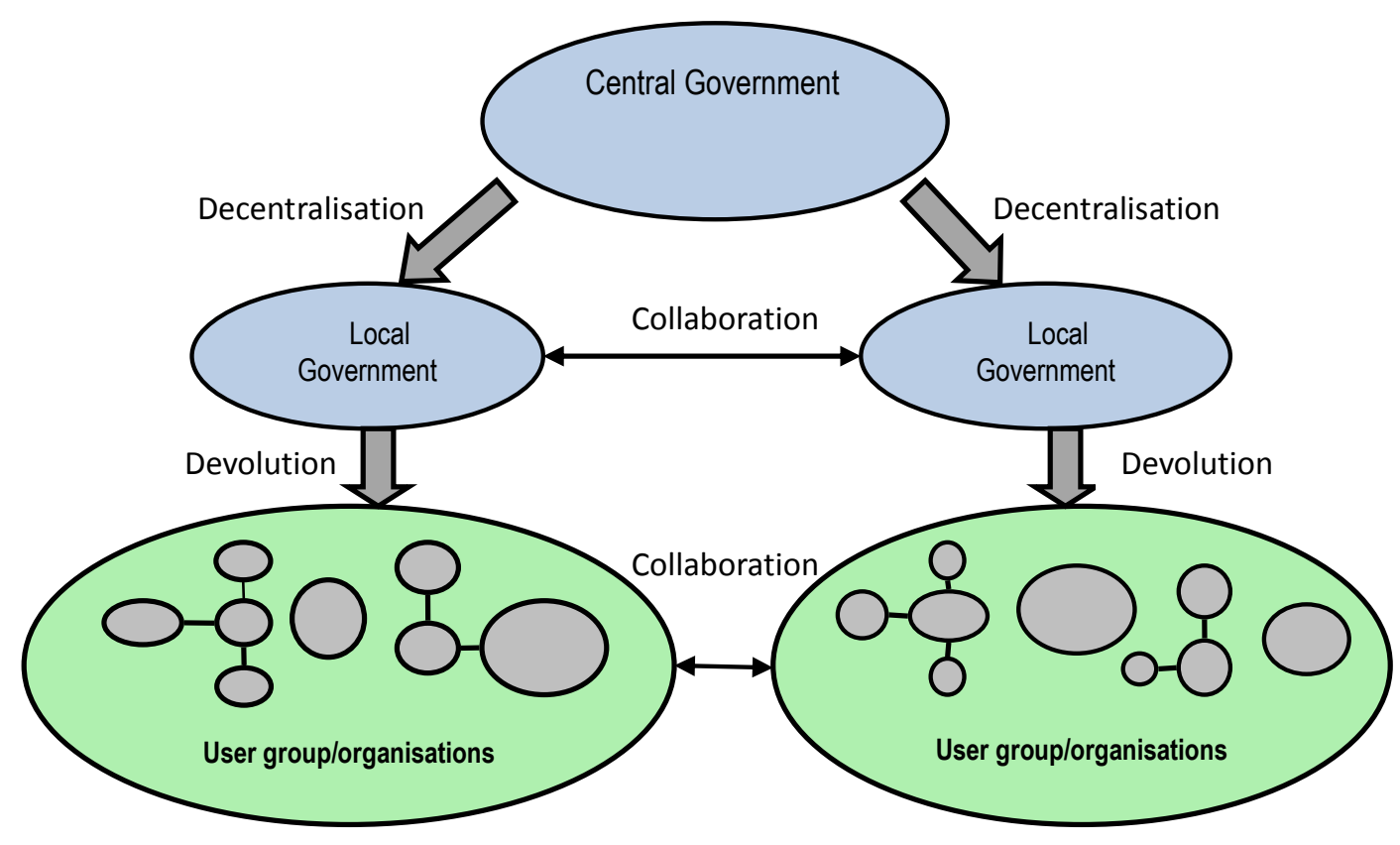

Figure 1.3 Decentralisation and devolution in resource and environmental management. Modified from Berkes, 2010: 491

This analysis helps to distinguish between "power sharing" among government agencies, and "power devolution" from the government to the resource users and their organisations. Since the late 1990s, there has been growing international interest in a civil society where the citizens are no longer treated as subjects, but as participants in governance (Berkes, 2010). Effective, genuine public participation in resource management has been recognised as an essential principle of good governance (Agrawal and Gibson, 1999; Ostrom et al., 1999; Ostrom, 2009).

The recent commons literature provides an important foundation for TEK conservation and indigenous rights protection as it recognises and emphasises the importance of resource users' rights and their participation in resource governance. As Agrawal and Gibsson (1999) point out, more collaborative governance may enable TEK holders as resource users to sustainably manage common resources over time. The power devolution that is advocated by many scholars in the commons field provides a great opportunity for the implementation of more collaborative resource management 
regimes in which local scale institutions (including indigenous institutions and TEK), can have a greater share in decision-making and management.

\subsection{Social-ecological systems and institutional linkages}

\subsubsection{Social-ecological systems (SESs)}

An important intellectual foundation of resource governance reform is the recognition of natural resource management as embedded within social-ecological systems. Socialecological systems theory recognises the inclusion of humans in the natural environment (Liu et al., 2007), and in turn reflects a more holistic view of the interrelationships among all components of a system. This approach is consistent with the way many indigenous communities understand their relationship with the environment (Berkes and Folke, 1998). As Ostrom (2009) points out, all humanly used resources are embedded in complex social-ecological systems, which are composed of multiple subsystems and internal variables (see Figure 1.4 below).

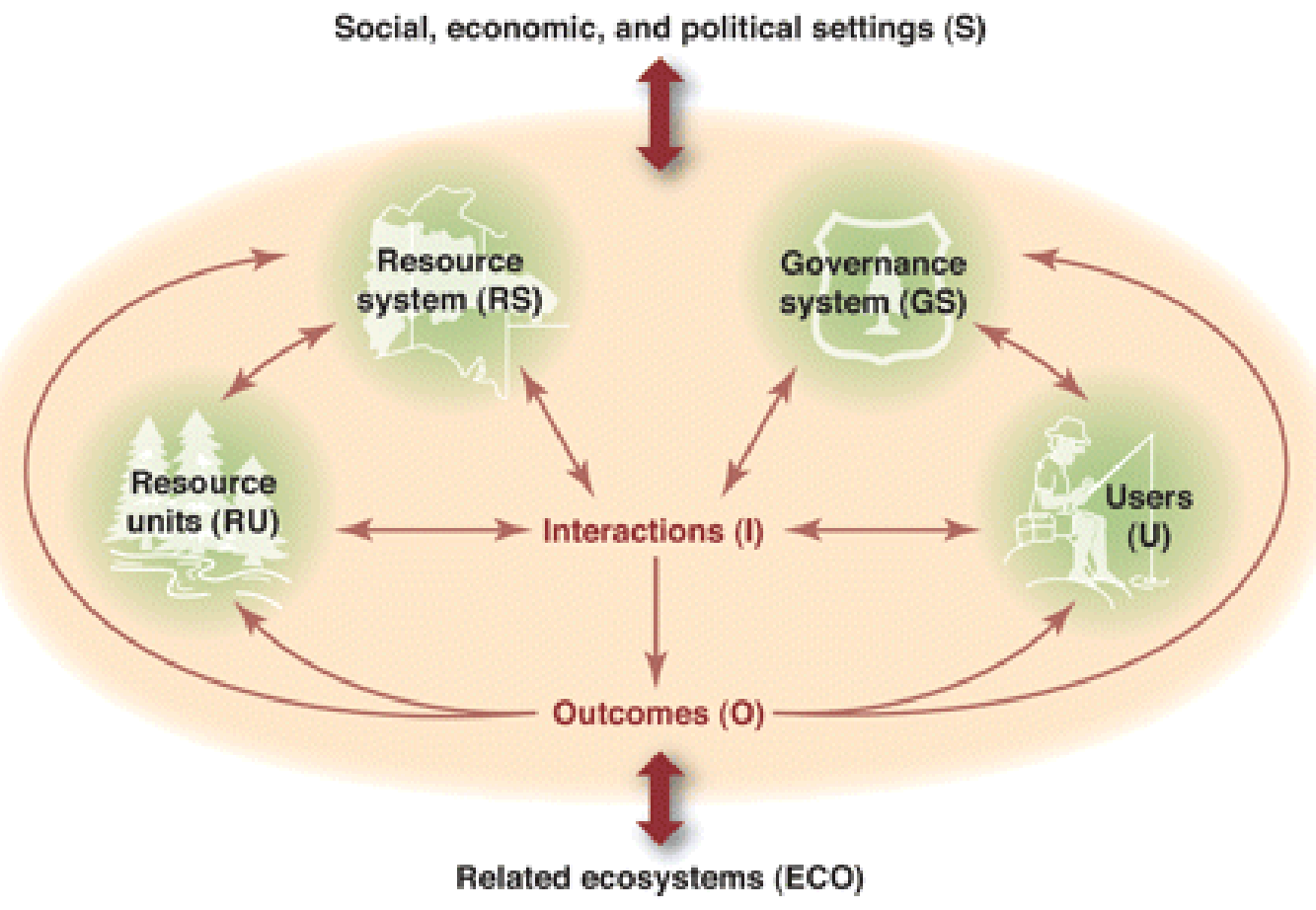

Figure 1.4 The core subsystems in a framework for analysing SESs.

Source: Ostrom, 2009: 420 
The above figure provides an overview of the relationships among four subsystems of a SES: 1) resource systems (e.g. a reserve encompassing a specified territory containing forested areas, wildlife and water systems); 2) resource units (e.g. trees, shrubs and plants contained in the reserve; types of wildlife; and the amount and flow of water); 3) governance systems (e.g. the government and other organisations that manage the reserve, the specific rules related to the use of the reserve, and how these rules are made); and 4) resource users (e.g. individuals who use the reserve in diverse ways for sustenance, recreation or commercial purposes) (Ostrom, 2009). Within this SES, subsystems affect each other; social, economic and political settings are constantly interacting with ecosystems. Each core subsystem comprises multiple secondary-level variables, such as the size of a resource system, mobility of a resource unit, level of governance, and users' knowledge of the resource system. All of these variables can be further refined into more discrete variables (Anderies et al., 2006; Ostrom, 2009).

Several key characteristics of SESs have important implications for my thesis research. First, SESs are dynamic; and complex interactions and feedback between natural and social systems are constantly occurring (Liu et al., 2007). This requires that effective management within the framework of SES has to engage various tools and techniques from both ecological and social sciences (Ostrom and Jassen, 2004). Inspired by this principle, the analysis of TEK threats and design of TEK conservation actions in this research applies an interdisciplinary approach in which principles and techniques from multiple disciplines are integrated, such as biodiversity conservation, resource management, education, policy and legislation and economic mechanisms.

Second, SESs are fundamentally multi-scalar. The numerous, complex relationships across different scales of space, time and organisational units occur continuously throughout an SES (Agrawal, 2003; Berkes, 2010; Ostrom, 2009). In accordance with this principle, addressing TEK threats and the implementation of TEK conservation must account for the need for cross-scale arrangements, including collaborations across different geographic regions and across different levels of institutional systems (see more discussion in next section). 
Third, as many scholars emphasise (e.g. Berkes et al., 2003; Folke, 2006; Liu et al., 2007; Ostrom and Jassen, 2004), uncertainty 5 is a key attribute of SESs. In any given system and any given time, the information and knowledge of the system will be incomplete. This uncertainty principle then informs practitioners and policy makers to shift from the conventional approach of "control and manage the change" 6 to adaptive management techniques centred on the concept of resilience ${ }^{7}$ (Berkes et al., 2003; Smit and Wandel, 2006; Walker et al., 2004). Adaptive management, characterised by learning-by-doing, is an iterative process of robust decision making in the face of uncertainty, which aims to maintain resilience over time (Berkes et al., 2000). It allows action without perfect information based on continuous monitoring and collecting more information, and adjusts the management approach as the system changes and/or our understanding of the conditions improves. The adaptive management concept is in accordance with TEK systems, which are dynamic and adaptive by nature; and is the only appropriate approach of treating TEK issues (Berkes, 2008). This adaptive principle has guided my analysis of the association between TEK threats and conservation actions, as well as action design in this research. For example, the research emphasises the great heterogeneity among different TEK systems, even within a TEK system; thus the conservation actions have to be designed adaptively based on specific local context and conditions, and continuously adapt to any changes in the local TEK system.

\subsubsection{Cross-scale institutional linkages}

Although the commons literature increasingly favours community-based approaches, much of the evidence from field research also illustrates that neither purely local-level management nor purely higher-level management works well by itself (Berkes, 2010). Management institutions should be designed and supported as multi-level systems that accommodate interactions and interdependency across scales ranging from the local

\footnotetext{
${ }^{5}$ Is a state of having limited knowledge where it is impossible to exactly describe the existing state, a future outcome, or more than one possible outcome of a given system.

${ }^{6}$ It assumes ecosystems are stable and near equilibrium, and seeks maximum yields from ecosystems.

${ }^{7}$ Resilience refers to the capacity of SESs to retain similar structure and functioning after disturbances for continuous development (Gunderson and Holling, 2002).
} 
level up to the national and international levels (Berkes, 2010). This is because different levels possess different capacities and provide different functions in the system (Bodin and Crona, 2009). For example, the conservation of migratory species requires crossregion institutional cooperation and cross-time arrangements (Epstein et al., 2009). Local resource users and their organisations must operate collectively to regulate the treatment of such species, sub-regional and regional agencies can contribute conservation measures, state governments can provide conservation policies and institutional arrangements that support local-level action, and in some cases international agencies can exert influence through international treaties and conventions (Agrawal and Ostrom, 2001).

The nature of cross-level resource use and management systems calls for institutional arrangements that are linked both vertically (across hierarchical organisations) and horizontally (across regions) (Ostrom et al., 1999). The vertical interplay of institutions influences how groups compete or cooperate for authority over resources (Young, 2006). Horizontal interplay reflects the spatial linkages within resource use system and the need for cross-region cooperation and arrangements (Ostrom, 1990). In addition, as many cases show (e.g. Berkes, 2010; Liu et al., 20007; North, 1990, 1991; Ostrom, 1990; Ribot, 2002; Swiderska, 2001, 2005), the threats to natural conservation and resource management come from multiple scales, therefore the solution needs to be multi-scalar in nature. Cross-scale institutional cooperation, in which governments, civil society and private actors are all involved in decisions, would be ideal for achieving effective resource management (Young et al., 2008).

Much of the literature states that cross-scale institutional arrangements can effectively facilitate collaborative resource governance in several ways. First, such arrangements facilitate the acquisition and exchange of different types of knowledge from different levels of the system (Bodin and Crona, 2009; Carlsson and Berkes, 2005). Second, by integrating the knowledge and skills held at different levels, cross-scale governance is more likely to support more equitable and reasonable resource allocation for effective outcomes (Hahn et al., 2006). Third, cross-scale governance facilitates the development 
and implementation of common rules regarding monitoring, sanctioning and conflict resolution among all stakeholders (Berkes, 2009; Scholz and Wang, 2006). These significant benefits discussed above have made a strong argument for the application of cross-scale institutional arrangements in TEK conservation.

\subsection{Political ecology and power relations}

Political ecology is a historical outgrowth of the central questions asked by the social sciences about the relationship between human society and nature (Berkes, 2008). Political ecology encompasses cultural and political activities within an analysis of ecosystems that are significantly, but not always entirely, socially constructed. The role of unequal power relations in constituting a politicised environment is a central theme of political ecology (Dobson and Eckersley, 2006; Robbins, 2012; Zimmerer and Bassett, 2003). Particular attention is given to the ways in which conflict over access to environmental resources is linked to systems of political and economic control first elaborated during the colonial era (Robbins, 2012). Many studies emphasise the increased marginality and vulnerability of the poor as an outcome of such conflict. The impact of perceptions and discourses on the specification of environmental problems and interventions is also explored, leading to debates about the relative merits of indigenous and Western scientific knowledge (Bryant, 1998).

Power relations, as many political ecologists point out (e.g. Bryant, 1998; Dobson and Eckersley, 2006; Robbins, 2012; Zimmerer and Bassett, 2003), hold a core importance for achieving sustainable resource management and effective community empowerment. The application of political ecology to indigenous knowledge issues, as Blaikie and Jeanrenaud (1997) indicate, has focused on the familiar political-economic divisions between stakeholders or interest groups; international, national and local interests; North and South; science and politics; officials and citizens; and power relations at the local level deriving from differences of class, ethnicity and gender. 
While the necessity of power decentralisation is widely agreed by many scholars, the actual processes of power sharing and power devolution have been achieved to varying degrees. As some scholars (e.g. Berkes, 2010; Ostrom and Janssen, 2004) suggest, the power of centralised management agencies should be redistributed and balanced, but not eliminated. However, centralised power should be used carefully because a lack of decision-making power by the lower levels of social organisations can make them vulnerable to the decisions that are made by the higher levels.

Many examples from the literature (e.g. Agrawal, 2002a; Case et al., 2005; Cruz García, 2006; Hill, 2004; Lizarralde, 2001; Robert and Angela, 2004; Ross, 2002a; Zent, 1999; Zent and Maffi, 2009) and from the survey conducted for this thesis (see Chapter 3) demonstrate that the loss of TEK and indigenous rights is largely caused by the political and cultural influence of dominant societies and economic stress. The power relations between different layers of complex political-social-ecological systems, especially between the community and government levels, have been a central concern in the areas of indigenous rights protection and TEK conservation (e.g. Bishop and Glynn, 2003; Kipuri, 2009). Such power relations determine who controls resource access and property rights (Collings, 2009), who makes decisions and how decisions are made (Barnett and Duvall, 2005), and even how the environment is framed via particular world views (Adler, 2005). The underlying fundamental question is how power relations within a society shape the interactions among people and institutions (Hurrell, 2005; Gruber, 2000). Unfortunately, too often, indigenous communities are in a powerless position. Indigenous self-determination with the aim of strengthening indigenous communities and reclaiming their traditional land and traditional rights has only been a recent movement since the last century. Short of revolution, the success of indigenous self-determination largely relies on policy, legislative and social support from the dominant societies (Adler and Bernstein, 2005; DESA, 2009).

For example, a case study from Mexico demonstrates how government support can promote indigenous self-determination. From the early 2000s, the Mexican state government recognises and protects a traditional property rights system called the 
"tenurial shell". By supporting community property rights and traditional institutional arrangements, the government supports the traditional belief structure and ecologically sustainable land use of the Huastec people (Alcorn and Toledo, 2000). In Canada, the Cree Amerindians re-established their traditional resource management institutions for beaver management in the James Bay area with the backing of government regulations (Berkes, 2000). However, there are many more cases which show that lower-level institutions, especially community institutions, are undervalued or even undermined by central government. For instance, the Chinese government had suppressed most traditional community-based institutions until the 1990s, and this led to the collapse of traditional institutions and the disempowerment of indigenous communities overall.

Achieving a completely well-balanced power structure between authorities and other stakeholders, especially community-level stakeholders, will remain a major challenge. The case study from Inner Mongolia that I have focused on as part of my thesis research provides an in-depth analysis of the recent institutional dynamics involving the government authorities and community organisations. This case reveals that the previous state-controlled resource management regime had led to resource degradation and generated social conflicts. However, willingness by the government authority to empower community-level institutions in local resource management can generate important benefits not only for resource restoration but also for social development, including indigenous empowerment.

In this research numerous community members, practitioners, researchers and scholars have pointed to the importance of power relations for indigenous rights protection and TEK conservation. As many survey respondents address, TEK degradation has largely been caused by the political, cultural and economic influences from the dominant societies that hold the power of decision making, leaving indigenous peoples powerless and marginalised. Indigenous self-determination has been a positive development in recent years; however, its success heavily relies upon authorities' recognition of indigenous rights and willingness to share power (Inglis, 1993). 


\section{AIM AND OBJECTIVES}

This thesis explores global patterns in the status of TEK, the threats that TEK faces, and the availability of TEK conservation actions. Based on a global survey and a literature review, this thesis also presents classification systems for TEK threats and TEK conservation actions that can be applied for case analysis and decision making in realworld situations. Based on a case study in Inner Mongolia of China, this research also examines the institutional dynamics between governmental authorities and community institutions, and explores the possibilities of utilising government policy and legislation to revitalise TEK at the community level.

The research undertaken for this thesis is organised into two major parts. The first part is based on a global survey. Its objectives include:

1. To explore any global pattern in TEK status.

2. To identify the threats faced by TEK globally and develop a TEK threat typology.

3. To explore the TEK conservation actions and develop a TEK conservation typology.

4. To explore the triggers and barriers to TEK conservation.

The second part consists of a case study of Inner Mongolia in China. Its objectives include:

5. To examine historical governmental policies and their effects on the traditional institutions and traditional herding practices in Inner Mongolia.

6. To examine the development and impact of community cooperatives for traditional herding practices and traditional institutions in Inner Mongolia.

7. To examine the triggers of and barriers to the cooperative organisations.

8. To explore the possibilities for revitalising TEK through the cooperative movements

9. To test the applicability of the TEK typologies developed from part one of this research.

The above nine objectives are developed to address the research gaps discussed in section 4.7 of this chapter. By satisfying these research objectives, this thesis will be able 
to fill these literature gaps and answers several central questions in the TEK field, such as what are the global patterns of TEK change? What factors are causing TEK loss? And how can TEK be conserved effectively?

\section{THESIS STRUCTURE}

This thesis is composed of seven chapters as follows.

\section{Chapter one - Introduction}

This chapter provides an overview of the whole thesis, including the concept of TEK, the significance of TEK, the status of TEK and TEK research, and the theoretical framework of the thesis.

\section{Chapter two - Methodology}

This chapter provides a detailed discussion of how the research has been designed and conducted and how the data have been analysed. The issues of positionality and ethic concerns are also addressed.

\section{Chapter three - TEK threats}

Based on a literature review, a global survey and follow-up interviews, this chapter discusses the status of TEK and causes of TEK change at a global scale, and presents a TEK threat typology. The typology includes definitions, explanations and detailed discussion with examples from the survey and literature review.

\section{Chapter four - TEK conservation}

Based on the same data sources presented in Chapter Three, this chapter discusses the available TEK conservation options at a global scale and presents a typology of TEK conservation actions. 


\section{Chapter five - Inner Mongolia case study}

This chapter presents a case study from Inner Mongolia in China. The study focuses on the institutional dynamics between the national authority and community organisations. It explores the possibilities for revitalising traditional institutions and practices under governmental policy and legislation.

\section{Chapter six - Typology application}

By applying the TEK typologies in the Inner Mongolia case study, this chapter aims to test the applicability of the typologies, and present a complete model for how to use the typologies in real-world situations analysis and project design.

\section{Chapter seven - Conclusion and Recommendations}

This chapter summarises the whole thesis and provides overall recommendations for further research in the TEK field. 


\section{CHAPTER TWO}

\section{METHODOLOGY}

In this chapter, I will provide a detailed discussion of how the research was designed, how it was carried out, and how the data were analysed. The issues of positionality and research ethics will also be addressed. As I discussed in Chapter One, this research comprises two major parts. Part one is based on a global survey and literature review; part two is based on a case study in Inner Mongolia, China. The methodological discussion in this chapter will addresses the treatment of philosophical concerns, ethical concerns and data analysis which applied jointly across both parts of the research. The methodology applied to research design and data collection for each part will be addressed separately.

\section{PHILOSOPHICAL CONCERNS}

\subsection{Positionality of the researcher}

A researcher's worldview, philosophical approach and experience have fundamental impacts on her/his research (Bryman, 2008). It is therefore important to define and analyse my position in relation to the research subject. In this research, my perspective is based on three basic attributes: 1) my cultural characteristics of being a Manchu Chinese; 2) my professional experiences and understanding of conservation, resource management and indigenous issues gained from working with four different nongovernmental organisations (NGOs) in China and New Zealand; and 3) a knowledge system developed from studying in Western academic institutions.

From my Chinese cultural background, I identify myself as a naturalist or Daoist, who believes in the harmonious relationships between nature (including all living and nonliving forms) and humans, and among human beings (Lai, 2003). This belief generates 
scepticism towards reductionist approaches in conservation and resource management practices, and sparks my research interest in more holistic worldviews and pluralistic approaches, such as institutional networks and participatory conservation and development. It also enables me to accept and apply the ideology and methodology of deep ecology and complex system theory, which recognise the interdependence and non-linear linkages among all living and non-living objects as a primary principle (Drengson and Inoue, 1995; Hammond, 2003).

My identity as a Man Tzu Chinese also has a special implication for the case study in Inner Mongolia. As Manchu ${ }^{8}$ share ancestry with the Mongolian people, it was relatively easy for me to be culturally accepted by the focal community. Furthermore, due to my previous work and research experience in Inner Mongolia (between 2004 and 2007), I speak basic Mongolian and understand Mongolian cultural traditions. This was advantageous for gaining community acceptance and recognition of me and my research. However, for in-depth communication in interviews and group discussions, I was assisted by a Mongolian/Mandarin translator.

Through my previous work and study experience in Western academic institutions, I have developed environmentalist views supporting the sustainable management of resources and stewardship of the environment through changes in public policy and individual behaviour (Meadowcroft, 1993). I am also significantly concerned with social equity and human rights. My previous research has shown me situations where people had a long history of sound stewardship of natural resources at the community level until they more recently lost their role in decision making. This finding triggered my current research.

It is also critically noteworthy that my use of qualitative methods for data collection and analysis could be affected by my previous experience and understanding. As Ezzy (2002) points out, pre-existing understanding and experience affect decision making regarding what to observe and what to do, and ultimately, affect the results. From all these

\footnotetext{
${ }^{8}$ An ethnic group which differs from the Han majority in China.
} 
perspectives, I am an outsider to the research communities. Applying my own knowledge and Western science-based worldview to judge traditional knowledge could lead to a bias in my research.

Although it is almost impossible to be an absolutely objective observer and researcher, the awareness of various potential biases may help to reduce the bias level and obtain truer results. In order to overcome the potential bias, I applied multiple research methods to test the data from different sources and to triangulate the results. During the coding process of data analysis, I followed a grounded theory which has been proven to effectively minimise a researcher's subjectivity in producing research results (Charmaz, 2006; Strauss and Corbin, 1997).

\subsection{Cross-cultural research and power dynamics}

As the aim of this research is to explore the global pattern of TEK and TEK conservation, it relies on communication with and learning from community members, researchers and practitioners across regions and across cultures. This research is then cross-cultural in nature and it faces specific moral and ethical challenges (Fons et al., 1997). Within cross-cultural research, power dynamics possess a central position of concern. As Smith (2005: 88) points out, “...research is not just a highly moral and civilised search for knowledge; it is a set of very human activities that reproduce social relations of power". The design of a research project, the methods for data collection and analysis, and the presentation of the results all reflect the power dynamics between researchers and the researched.

As many scholars critique, the scientific research of indigenous knowledge has been shaped by unbalanced power structures (e.g. Agrawal, 2002; Smith, 1999, 2005). The structure of such research has often favoured the position of the researcher, who in some cases has been implicated in Western colonialism (Smith, 2005). Most of the indigenous research has been carried out by Western researchers, not the indigenous people themselves. Therefore, the interpretation and presentation of indigenous 
knowledge in academic and public publications are more Westernised, if not colonial (Smith, 1999). An intellectual foundation for this phenomenon might be that, "It is difficult for people from 'advanced' cultures to accept the idea that people from 'primitive' cultures might know something scientifically significant, or even know more about a subject within the field of natural science than do scientists" (Lewis, 1989:78).

I am aware that my Western academic background could make me less sensitive to TEK systems and values, despite my full willingness and intention to acknowledge them. Where Western science and TEK are rooted in different worldviews, methodologies and power structures (Berkes, 2008), it can be challenging for Western researchers to access, interpret and present TEK without bias.

\section{ETHICAL CONCERNS AND ETHICAL APPROVAL}

The Code of Ethics of the International Society of Ethnobiology (2006) provides the ethical foundation for this research. The Principles and Practical Guidelines of the Code "are based on the concept of traditional resource rights. They facilitate compliance with the standards set by national and international law and policy and customary practice, and recognise traditional and customary laws, protocols, and methodologies extant within the communities where collaborative research is proposed." The Code specifically emphasises the creation of sincere partnerships and collaborations between researchers and indigenous participants. I am a member of ISE and my research is consistent with its vision and Code of Ethics.

Furthermore, in order to avoid any potential harm to the research participants and any other possible ethical issues that could arise from my research, I applied for, and was granted ethics approval by the Human Ethics Committee of Victoria University of Wellington (Approval No. 17360) ${ }^{9}$. Research Information Sheets were given to, and

\footnotetext{
${ }^{9}$ See the Approval in Appendix 3.
} 
Consent Forms ${ }^{10}$ were signed by, the participants before an interview or a group discussion was initiated. All interviewees' identities remain confidential, unless they indicated otherwise. A summary of this research will be returned to interviewees who requested it on their consent forms.

\section{RESEARCH DESIGN}

As indicated in Chapter One, this research aims to explore global patterns in the status of TEK, the threats that TEK faces, and the availability of TEK conservation actions. The research comprises two parts with nine objectives (see Chapter 1, section 6). The research methodologies were designed separately for each part.

\subsection{Part one - Global survey and literature review}

A mixed qualitative and quantitative approach was employed in this part of the research, with three research methods used: literature review, questionnaires and semistructured interviews. The research started with a comprehensive review of academic literature and literature from relevant international agencies, governmental agencies and NGOs regarding TEK issues. The key words used for the research were traditional ecological knowledge, indigenous knowledge and traditional knowledge. Three search engines were used: Web of Knowledge, Science Direct and Google Scholar. In addition, numerous organisational websites were searched, such as websites of United Nations, International Union for Conservation of Nature, World Bank, International Institute of Environment and Development, etc. ${ }^{11}$ After a review of 70-80 articles and books, a number of key studies were scoped according to their relevance to the research topic (i.e. TEK status, the causes of TEK change and TEK conservation). By applying a snowball technique, additional relevant studies were identified through reviewing the references cited in these key studies. In total, 152 studies were reviewed. This review

\footnotetext{
10 See samples of the Information Sheet and Consent Forms in Appendix $1 \& 2$.

11 See Appendix 4 for a list of the targeting organisations.
} 
provided a framework for my research design, and helped me to identify and explore several literature gaps in the TEK research field.

Because the primary goal of this part was to explore global TEK patterns, surveying was chosen as the main research tool. This is because surveying is commonly considered to be useful when collecting data regarding phenomena that a researcher cannot observe directly in person (Bryman, 2008). It also enabled me to extend the breadth of my research beyond what is available in the literature. The questionnaire was developed in three versions that were designed for different target groups: community members (TEK holders), TEK-focused researchers and TEK-focused organisations. These three versions were based on the same central questions, such as:

- Based on your experiences, how would you assess the general change in TEK in your location over the last 50 years?

- In your opinion, what are the main reasons for TEK change in your location?

- What has been done to conserve TEK in your location?

- How successful have these TEK conservation practices been in your location, and why?

What differed across versions were the questions about respondents' basic attributes, such as their location, their length of residency or research duration in the location, and their institution if applicable (see samples of the questionnaires in Appendix 5).

Originally, the survey was designed to target indigenous community members worldwide to gain first hand information about TEK, TEK change and its conservation. The information obtained from TEK-focused researchers and organisations would then provide supporting information about the research objectives. However, after the piloting period, I realised that community members were far less accessible than the other target groups due to the financial and logistical constraints of my research. For example, the primary survey tools of this research were email and internet communication. Indigenous communities in many regions do not have access to such tools. I also handed out about 100 copies of my questionnaire in person during multiple 
international conferences. However, there were only few indigenous community members who could attend these conferences, compared with the number of researcher attendees. I met a certain number of community members at the conferences, but the representativeness and geographical coverage of these participants were limited. Thus, I adapted the main target group to also include TEK-focused researchers. It is undeniable that shifting the main research target may have generated bias in the results. This is because researchers have different perspectives from community members in terms of their understanding of TEK and the observation of TEK change and conservation. Researchers and organisational practitioners tend to have an outsider's view (etic), while the community members have an insider's view (emic). However, the expected benefits to the research outcomes from expanding the breadth of the survey were an overriding consideration.

The response rate is a major concern for any survey research (Bernard, 2011). In order to maximise the response rate, I applied several principles during the research design and data collection process. First, I used plain and easy-to-understand language in the questionnaire to avoid potential misunderstanding. I also minimised the number of questions so the questionnaire could be completed in a reasonable amount of time. The questionnaire was always accompanied by an information sheet which explained the research in detail. During the data collection, I also applied a procedure called "five compatible contacts" of the Tailored Design Method (Dilman, 2000): brief pre-notice, questionnaire, thank you contact, replacement questionnaire, and final contact. This procedure has also been shown to increase the response rate (Bernard, 2011).

In order to gain in-depth understanding of the questionnaire responses, I also asked each participant to indicate at the end of the questionnaire, whether or not he/she would be willing to participate in a semi-structured interview.

\subsection{Part two - Case study}

A qualitative research approach provided the foundation for this case study. There are 
two main reasons for choosing a qualitative research approach. First, a qualitative approach enabled me to make in-depth connections and build trust with participants, and to obtain in-depth information about the research subjects (Iain, 2010; Punch, 2005). Building trust was a major objective in my research. I spent two months with the focal community in Inner Mongolia, taking part in participants' daily lives and their community activities in order to build trust. As my research progressed, Mongolian herders began sharing their cultural knowledge and life stories with me. Developing trust and mutually respectful relationships with the participants over a longer period of time was preferable to a rapid and impersonal quantitative survey technique. It enabled the collection and assessment of more diverse, complex and unanticipated information than would have surfaced under a rigid quantitative approach. Another reason to choose a qualitative approach was its suitability for application by an individual researcher with limited ability to canvass a wider area to achieve statistically significant results.

As Bernard (2011) addresses, the use of multiple research methods can enable researchers to overcome the limitations of each individual method. I therefore applied a mixture of four methods to triangulate the results: literature review, semi-structured interviews, focus group discussions and participant observation. Triangulation has been recognised as a powerful technique to facilitate validation of data through cross verification from multiple sources (Bogdan and Biklen, 2006).

The literature review comprised two aspects: 1) review of Chinese government documents and academic articles regarding government policies that have affected grassland management since the 1950s, including the recently established Cooperative Law; and 2) academic articles about traditional and current rangeland management in both Inner Mongolia and wider regions of China. The key words used for the literature review included traditional ecological knowledge/indigenous knowledge + Inner Mongolia, traditional ecological knowledge/indigenous knowledge + China, grassland management + China, cooperative movement, and Cooperative Law. Four search engines were used: Web of Knowledge, Science Direct, Google Scholar and Wanfang Academic Database [in Chinese]. Relevant government websites (e.g. websites of China State 
Government, Ministry for Agriculture of China, Inner Mongolia Government, etc.) were also targeted as they provide information on historical policies and the Cooperative Law and its relevant measures. The literature review provided useful background information about the historical and current policies regarding rangeland management in Inner Mongolia.

In practice, semi-structured interviews and focus group discussions were employed and provided flexibility to my research. For example, when I visited a household for an interview, I often found there were more herders than I expected. There were either relatives or friends who were visiting the target family. Under Mongolian cultural tradition, relatives, friends and neighbours visit one another on a very frequent basis with no need for appointments. When the number of herders was more than five, I tended to organise focus group discussions instead of planned interviews. The interviews and focus group discussions used the same set of questions. Information collected through participant observation supplemented the interviews and focus group discussions.

Note: The detailed description of the research site is provided in Chapter Five, section 2.

\section{DATA COLLECTION}

\subsection{Part one - Global survey and literature review}

Before the survey was officially launched, a two-month pilot was carried out in early 2010. Twelve key contacts for the pilot were identified from the literature review and my network. Their expertise in the fields of TEK and indigenous issues was mainly identified through their publications and their knowledge of the topics. The questionnaire was sent to these 12 key contacts and their feedback was discussed either in person or via phone conversation. Continuous discussions with my supervisor and colleagues also helped to generate useful feedback. 
The survey was launched in early May 2010 at the $12^{\text {th }}$ International Congress of Ethnobiology in Canada (http://www.tbgf.org/ice). The conference gathered indigenous community members, conservationists and conservation and development organisations from more than 50 countries around the world. Attending and presenting at the conference provided me with a great opportunity to meet potential participants, explain my research and hand out the questionnaire in person. The survey was closed on 31 May 2011. Within this one-year survey period, two main approaches were applied for data collection:

1) Distributing the questionnaire in person at international conferences, including the

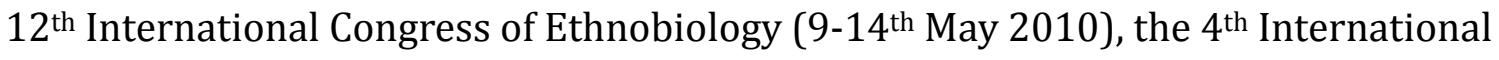
Conference of Indigenous Knowledge (June 2010, http://www.traditionalknowledge 2010.ac.nz) and the Sharing Power Conference (Janurary 2011, http://www. sharingpower.org). Those conferences were chosen because of their wide geographical coverage of participants and their relevance to the research topic. However, due to the difficulty of arranging international return post, this "handing out" method actually functioned more like a research pre-contact (or research advertisement). The majority of the participants preferred the online survey tool or email.

2) Email and online survey tool. Based on the literature review, online searching and networking, I developed a list of potential survey participants, including researchers, organisations and community members. The contacts from multiple conferences added a significant number of survey targets to the list. I set up an online questionnaire using a survey engine - Qualtrics (https://vuw.qualtrics.com/ControlPanel, that was provided by Victoria University of Wellington. I then emailed the link for the survey or the questionnaire itself to the potential participants. The survey was published online ${ }^{12}$, and I also encouraged participants to distribute it within their networks. The actual number of people who received the link or questionnaire was unknown because the link was passed on through research networks, which precluded an accurate calculation of the overall response rate.

12 The questions remained the same regardless of whether on paper or online. 
The survey generated 216 responses, of which 137 completed the minimum effective questions $^{13}$. Of these effective responses, 102 were from researchers, 26 were from community members, and nine were from organisations. Considering the limited sample size and uneven number of responses from the different target groups, I chose to aggregate the results across respondent groups instead of differentiating them by source.

After receiving a response, I replied within three working days. For those who agreed to be interviewed ( $n=70,51 \%$ of the questionnaire respondents), I contacted them to arrange a semi-structured interview. Due to the limitation of time availability and accessibility, only 46 (66\%) of them were interviewed. Three different interview methods were used: face-to-face $(n=19)$, via phone call $(n=12)$, and via internet communications ( $\mathrm{n}=15)$ (Skype voice or video calls).

There was also a second round of interviews, this time focused on the typologies. Once I developed a draft of the typologies, I sent it to 20 key contacts identified from the survey interviewees for an initial review. These key contacts had provided detailed information in response to the survey questions, and expressed further interest in commenting on the draft of the typologies. Another consideration when choosing these reviewers was their ability to represent the perspectives of different stakeholders regarding the final typologies. Seventeeen of them reviewed and commented on the typologies, including eight community members from Canada, China, Nepal, New Zealand and USA, and nine experts in various fields. These experts included a world-leading expert in TEK and natural resource management, an expert in indigenous rights with a global focus, a practitioner in intellectual property rights with a global focus, a scholar specialised in indigenous knowledge and scientific research, and other experts in ethnobiology, ethnobotany, conservation biology, integrated conservation and development,

\footnotetext{
${ }^{13}$ In the Researcher and Organisation versions of the questionnaire, the minimum effective questions were questions 1, 4, 5 and 7; in the Community version, the minimum effective questions were 1, 5 and 7. See Appendix 5 for examples.
} 
community-based resource management and community empowerment. The typologies were then modified according to their feedback.

\subsection{Part two - Case study}

\subsubsection{Semi-structured interviews ( $n=91)$}

Semi-structured interviews were used as a main research method for the case study, as the method provides in-depth information and is commonly used in qualitative research (Bernard, 2011). I targeted three different groups of respondents: community members $(n=68)$, rangeland management experts $(n=11)$ and governmental officials $(n=12)$.

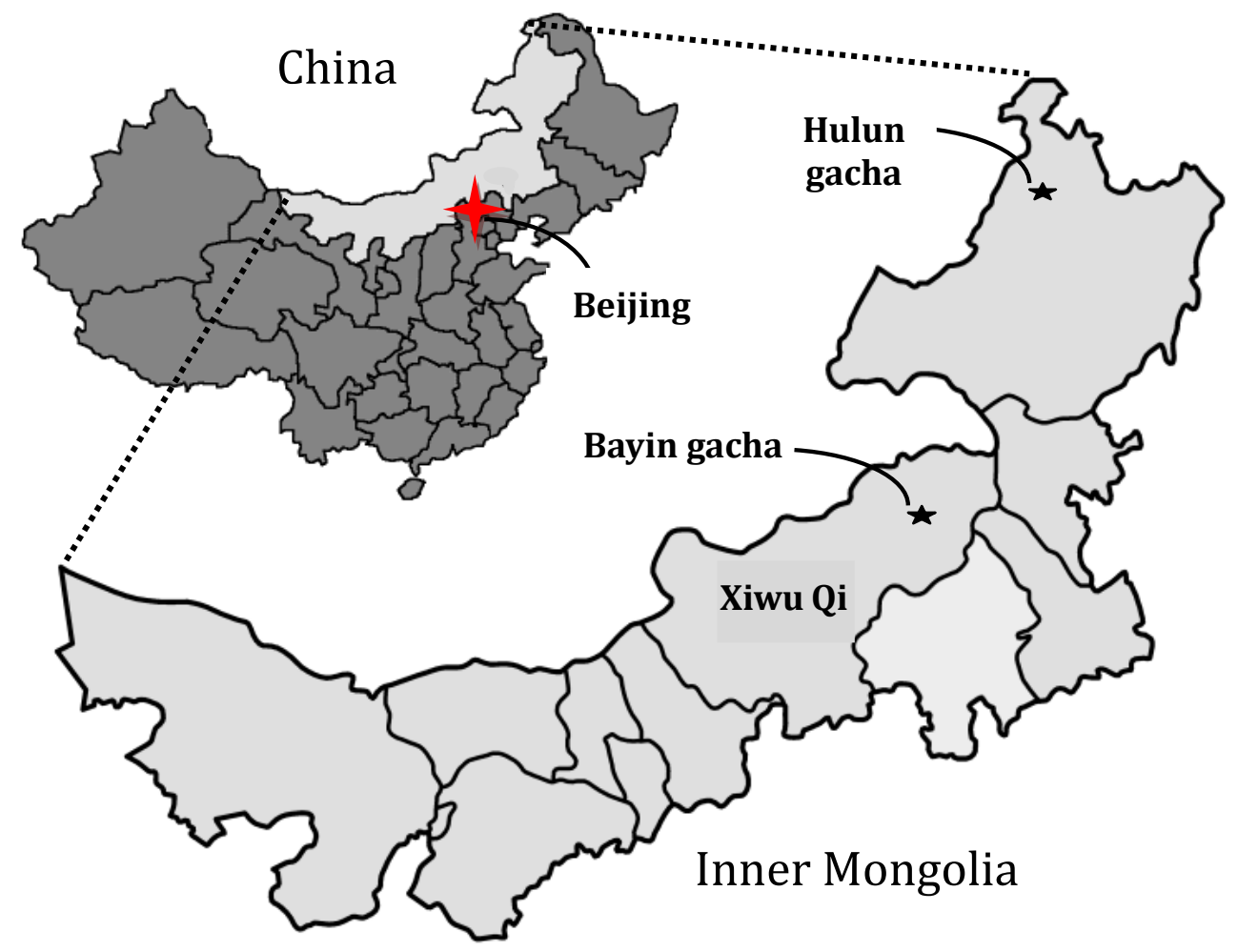

Figure 2.1 The locations of two target communities in Inner Mongolia, China. Modified from Croquant, 2007

The adult community members in the Bayin Wenduri gacha (gacha means village in Mongolian) (short as Bayin gacha below) were the main target of the research (see 
Figure 2.1). Bayin is a typical Mongolian community with the vast majority (90\%) of its population engaged with herding, and only $10 \%$ of them working in town or other cities. Among the current population of 290 (in 83 households), indigenous Mongolian herders accounted for the vast majority $(97 \%, n=282)$, while Han immigrants ${ }^{14}$ only accounted for eight individuals. The interviews, in association with focus group discussions, were originally intended to target all households in the community. However, during the research period, there were only 63 accessible households $(n=252)^{15}$; others worked or lived in town, or herded in other areas. In total, 68 herders were interviewed (39\% of the accessible, effective residents). Focus group discussions engaged a further 64 residents (36\% of accessible, effective residents) ${ }^{16}$ (see Figure $2.2 \& 2.3$ ).

Interview questions included the following:

- How do you carry out herding?

- Is there any cooperation among community members?

- How much do you remember of the traditional herding practices, especially the nomadic herding practices?

- What was the social structure among community members like in the past?

- Is there any cooperative organisation in the village? What are your attitudes toward it?

Note: A list of interview questions is provided in Appendix 8.1.

In addition to the research-site interviewees, I also interviewed two leaders from another cooperative: Hulun Be'er Pastoralism Cooperative (Hulun cooperative) in Hulun gacha, located $400 \mathrm{~km}$ northeast of Bayin gacha (see Figure 2.1). The cooperative was established in 2009, and achieved village-wide cooperation involving 44 households. Hulun cooperative provided supplementary information for the case study about how cooperative organisations operate.

\footnotetext{
14 The two Han families moved into the community in the 1950s.

15 Not all of the accessible residents accounted for effective interviewees, as about $30 \%(n=76)$ of them were under age 18 and therefore were not the target of the interviews. There were 176 accessible, effective interviewees. 16 In total, $75 \%$ of the accessible, effective residents participated in this research.
} 
In order to gain an understanding of the Cooperative Law's history and perspectives from policy makers, I also interviewed twelve government officials in Beijing and in the local government in Inner Mongolia. There were two major focus points for the data collection from officials: 1) how did the Cooperative Law originate? and 2) How has the law been implemented? Based on the literature review and my networking, I found two interviewees who were in charge of drafting the law from the Ministry of Agriculture in Beijing, and interviewed them. Also through my networking, I was able to access and interview ten officials from the Industry and Commerce Bureau who were in charge of implementing the law. Four of them were from the national bureau, and six of them were from the local bureau (see the question list in Appendix 8.2).

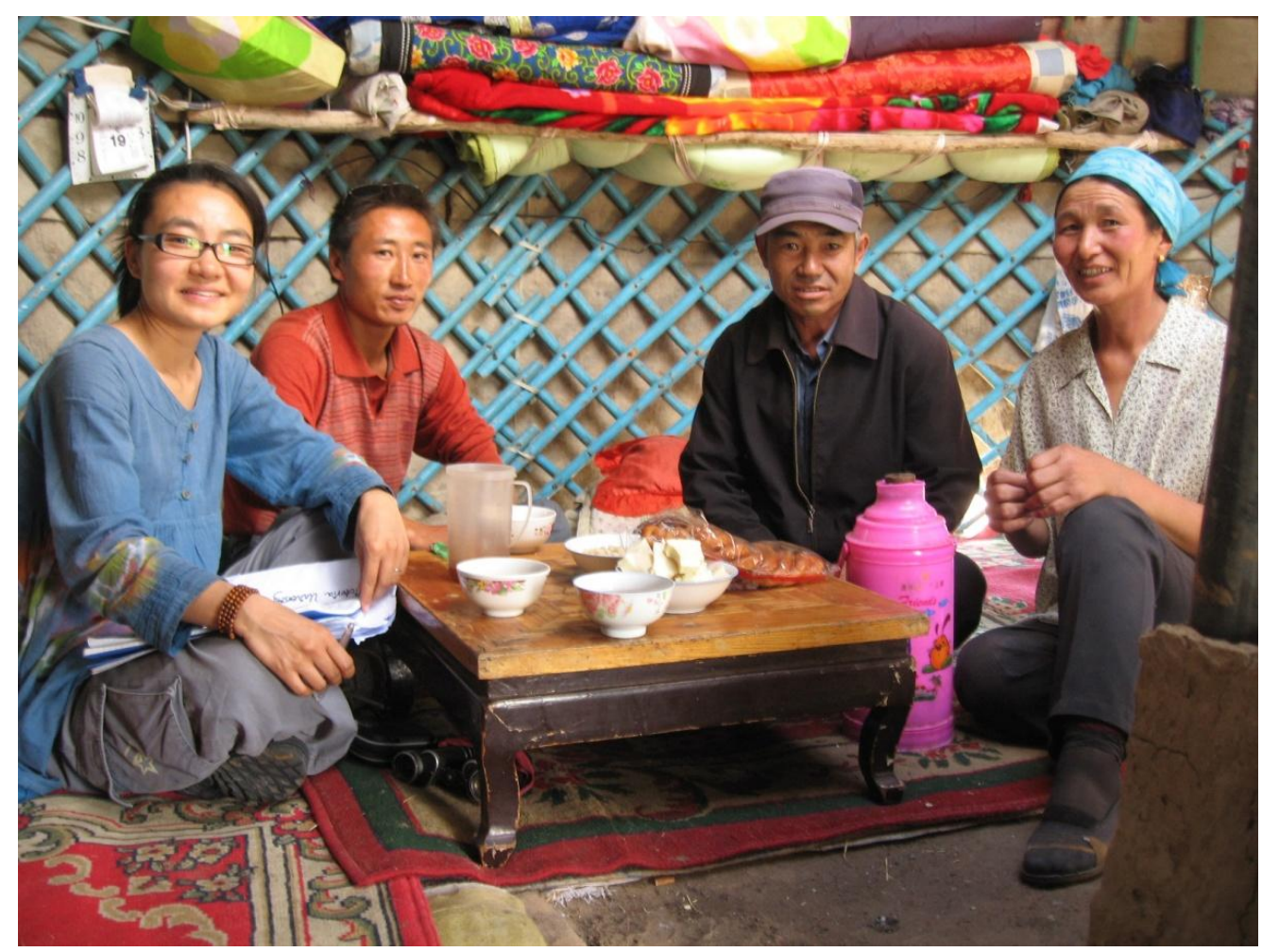

Figure 2.2 An interview conducted in a household in Bayin gacha (photo by: Sarengaowa, 2010) 


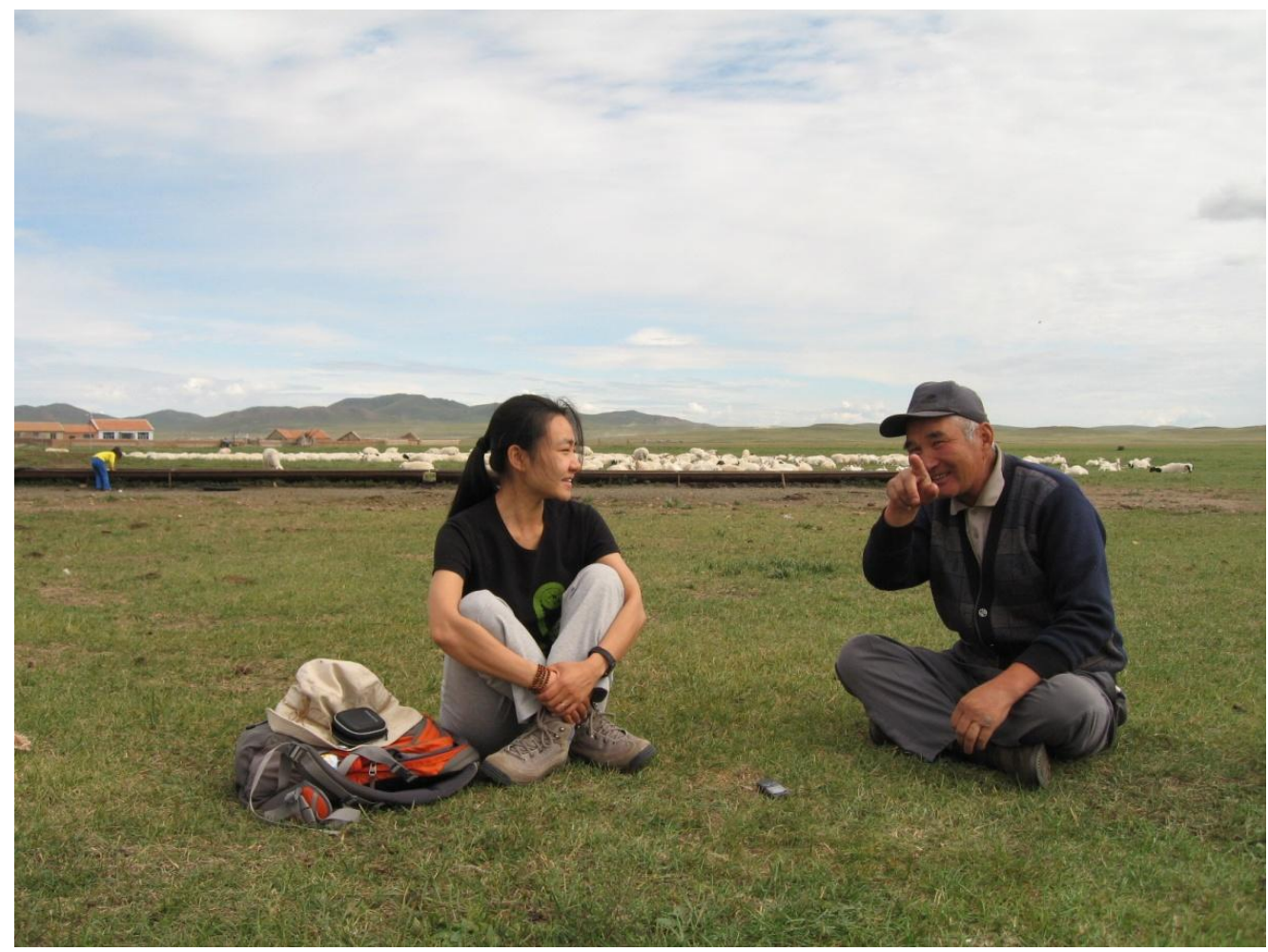

Figure 2.3 An interview conducted in the field in Bayin gacha (photo by: Sarengaowa, 2010)

I also interviewed 11 experts in grassland conservation, Mongolian traditional culture and practices and community-based conservation and development in Beijing and Inner Mongolia. Among them, two were academics in rural resource management, five were academics in grassland ecology, two were hired as experts in Mongolian traditional culture and practice for a local NGO in Inner Mongolia, and two were NGO practitioners in community-based conservation and development. I first identified 15 experts from the literature review and my pre-existing contacts. After initial contacts, 11 of them agreed to be interviewed. All of these interviews were face-to-face. The information provided by these interviewees mainly focused on their knowledge of traditional natural resource management, traditional institutions, the recent changes in these factors, review of governmental policies and their effects on community resource use and institutional change (see the question list in Appendix 8.3). 


\subsubsection{Focus group discussions ( $n=5,64$ participants in total)}

Compared with interviews, focus group discussions use group interactions to generate data. Instead of asking questions of each person in turn, I encouraged more interactive participation. Participants talked to one another, exchanged ideas and information and commented on each other's points. In this way, participants had more active involvement in the research (Kitzinger and Barbour, 1999). Also, the data collection was more time efficient relative to that for individual interviews. Five focus groups were organised during the research, in which a total of 64 individuals participated (see Figure 2.4). Focus group discussions also fitted the Mongolian culture and community setting at the research site. Traditionally, intra-community contacts are very strong. Visits and gatherings across families happened on a daily basis. I found herders were more communicative in a group setting than in an interview setting.

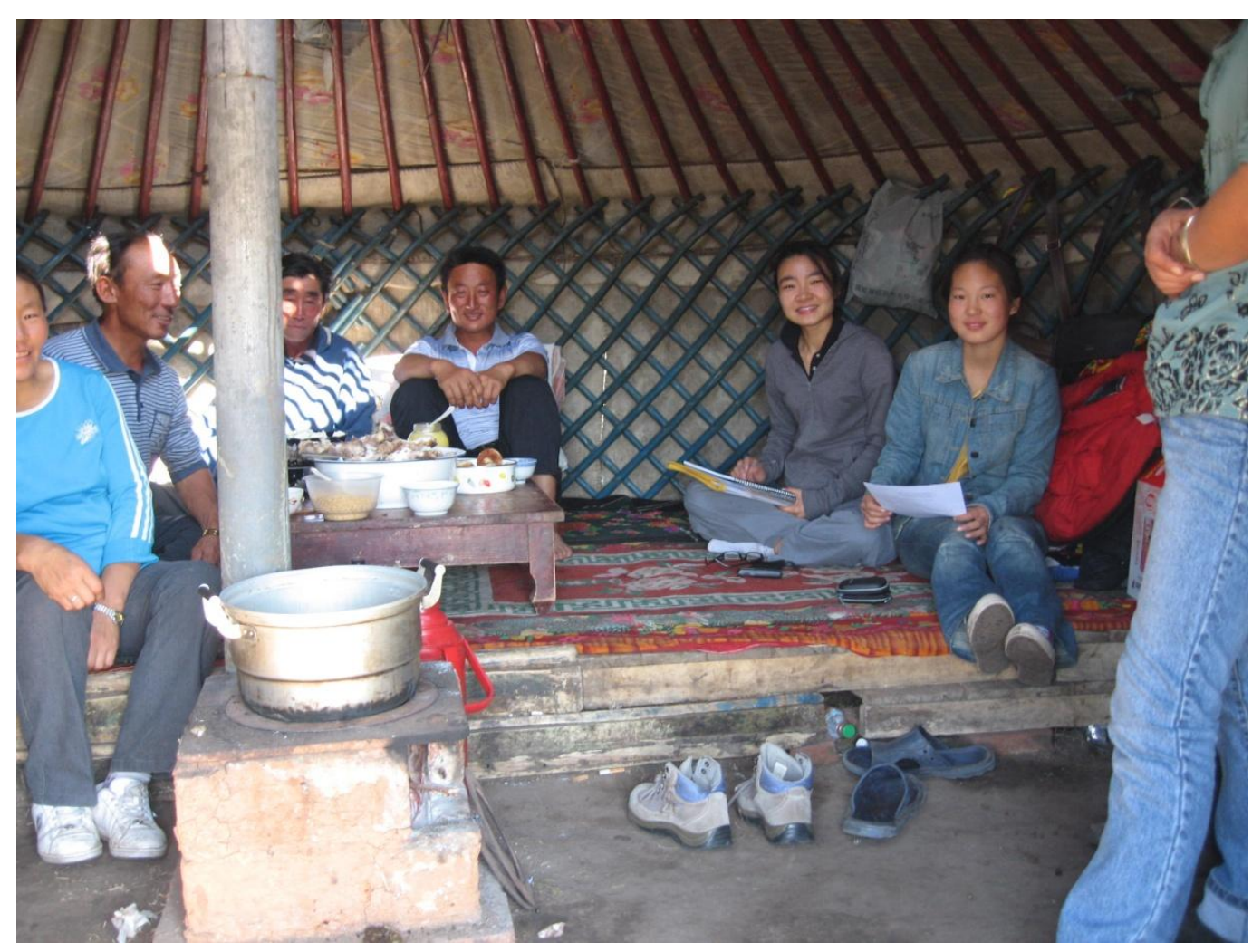

Figure 2.4 A group discussion conducted in a local household (photo by: Wuritu, 2010) 


\subsubsection{Participant observation}

Where a gap can arise between stated and actual behaviours, observation can play a significant role in discovering the actual behaviours of a focal population (Silverman, 2005). As a researcher, I lived in Bayin community for eight weeks ${ }^{17}$. This meant that I participated in members' daily lives and had the chance to observe their actual herding activities, cooperative practices, and community social structure through their gatherings and activities (see Figure 2.5). I also had informal conversations with members on many occasions. The observation contributed a significant amount of information that did not arise through interviews and/or group discussions and added to the final results.

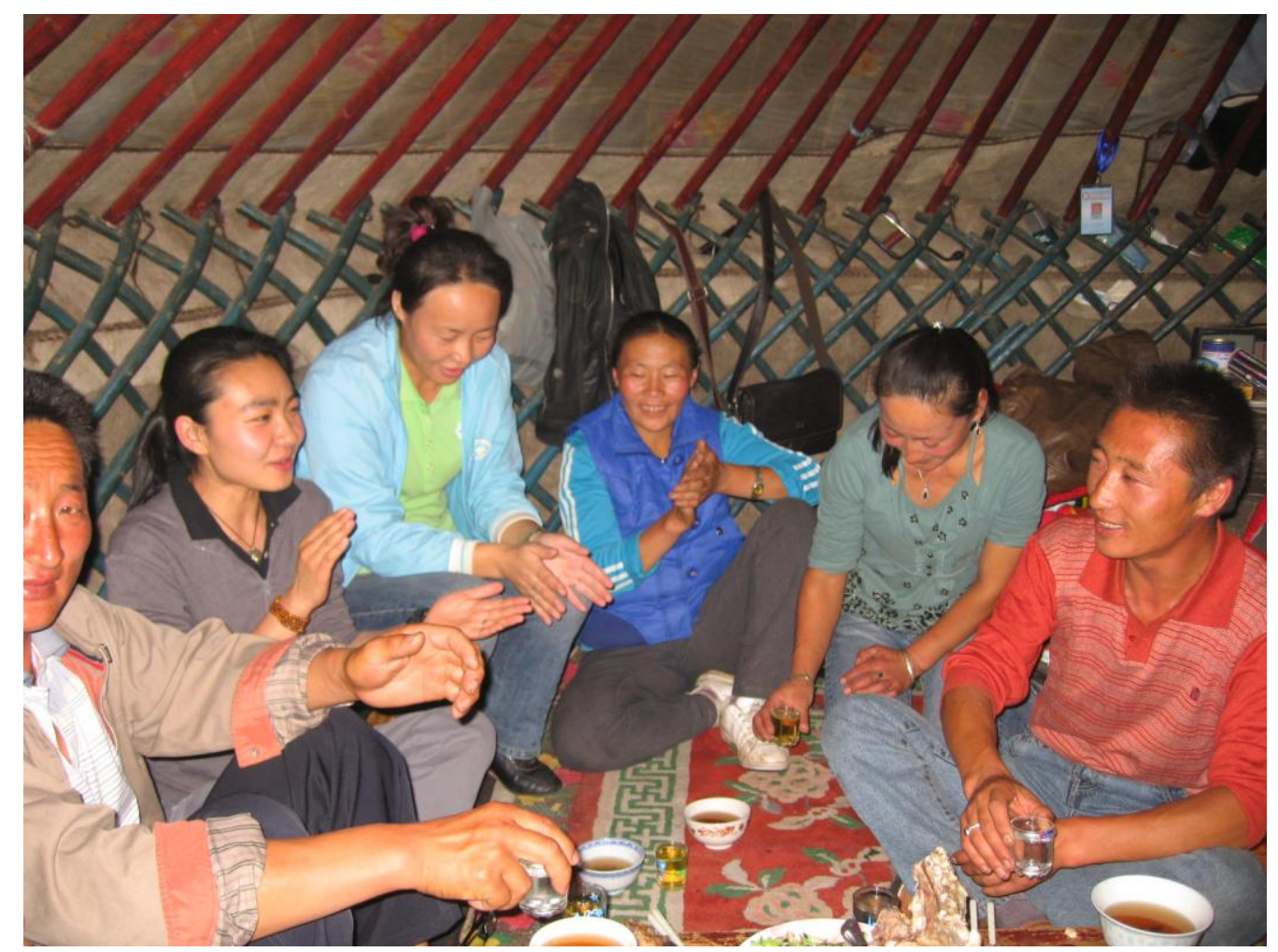

Figure 2.5 Participant observation conducted during a community gathering with seven families (22 people) (photo by: Bayaer, 2010).

\footnotetext{
${ }^{17}$ Previously, I lived and worked in Inner Mongolia for about six months between 2004 and 2006. This experience gave me a good understanding of Mongolian culture, livelihood, lifestyle, landscape and natural resources.
} 


\section{DATA ANALYSIS}

\subsection{Qualitative data}

The research involved four sources of qualitative data: 1) the answers to open-ended survey questions; 2) records and notes from interviews; 3 ) records and notes from focus group discussions; and 4) notes from participant observation. The data analysis was based on the grounded theory approach and its coding procedure. Grounded theory allowed me to follow an inductive approach to building a perspective on the research outcomes. As Strauss and Corbin (1990:23) suggest, grounded theory can be carried out when "one does not begin with a theory, then prove it. Rather, one begins with an area of theory and what is relevant to that area is allowed to emerge". The study of TEK differs from the conventional Western research approach of hypothesis (before going into the field) and validation (in the field) (Lalonde, 1993). Wolfe et al. (1991) provide a comparison between the TEK knowledge system and modern scientific knowledge system. There are several fundamental differences between them. TEK is commonly based on a holistic worldview. It then positions humans as subordinate to nature. On the contrary, the Western scientific approach is based on a reductionist worldview, and recognises humans as being able to play a dominant role over nature. Accordingly, TEK is presented and communicated through intuitive, oral and spiritual forms (e.g. oral history and rituals), while Western science follows an analytical and literary/academic approach (e.g. hypothesis and laws). Reflecting these fundamental differences, the research and analytical methods applied to TEK can be expected to differ from the conventional scientific ones.

Grounded theory was also a critical tool to help avoid potential bias and misunderstanding related to local knowledge. For example, following a grounded theory approach, I tended to avoid any hypothesis or prediction before collecting data. During the data analysis, the results were produced based on the data collected from the survey only. This helped to remove the bias that stemmed from my own perspective and other literature (Charmaz, 2006). 
There were three sources of data that contributed to the formation of the typologies: the survey results, the literature review and the results from the case study in Inner Mongolia. In contrast to methodology applied to the quantitative data analysis (e.g. on the geographic distribution of TEK studies and overall trends of TEK change) which was conducted separately from the literature review and the survey, I decided to combine the qualitative data from different sources to form unified TEK typologies. This is because the typologies support a comprehensive understanding of all possible threats to TEK and all available conservation actions at the global scale. Thus, there is no point in separating the results from the survey, the literature review and the case study for the purpose of differentiation or comparison; rather, they supplement one another and contribute to the formation of the synthesised typologies. To create the typologies using these diverse sources of information, I applied the three-step coding procedure of grounded theory, i.e. open coding, axial coding and selective coding (Strauss and Corbin, 1997). For example, to analyse the data regarding direct threats to TEK:

- $\quad$ Open coding - I broke down, examined and compared the original data in order to abstract and conceptualise it. Then I grouped the data into categories.

- $\quad$ Axial coding - According to causal conditions and intervening conditions, I reassembled the data in new ways and identified connections between categories.

- Selective coding - This was the final part of the coding procedure. It was also a further step of axial coding with more abstract selection. I eventually selected six categories and produced my own grounded theory related to the research topic. Adjustments were also made according to the feedback from 17 experts.

\subsection{Quantitative data}

For the quantitative data which were generated from the survey and the literature, I applied descriptive quantitative statistics to present the data in aggregate by using Qualitrics data analysis tools (an online survey tool) and Microsoft Excel. I used this approach to analyse variation in: 1) responses' geographical distribution; 2) the overall trend of TEK change; and 3) the significance of different categories of TEK threats and conservation options. 
For all three targets, the first step was to tabulate the data for the different variables (e.g. different geo-regions, different scales of TEK change, and different categories of TEK threats and conservation options). Then percentage distribution was applied to reveal the geographical distribution of survey responses, and frequency distribution was used to present the trends for TEK change (Robson, 2002) ${ }^{18}$. After using qualitative data analysis to develop the categories of the TEK typologies, the data for each category of TEK threats and conservation actions were disaggregated, and ratios among these categories were produced (Bernard, 2010; Bryman, 2008). These ratios represent the significance of each category identified by the survey respondents and in the literature.

\section{LIMITATIONS}

The most significant limitation of this research was the limited number of survey responses. Ideally, the survey should be carried out evenly in each country or region and should seek ample representation from all key stakeholder groups: community members, researchers and organisations. This was not possible due to financial, logistical and time constraints of this research. As an individual PhD student researcher, my capacity to reach potential respondents was limited mostly to internet communication and international conferences. As a result, I have to be very careful to qualify conclusions about the global patterns produced from my research. Even though the responses to the survey were from 48 countries and regions, the representation from each country and region was limited. For example, the survey had 34 responses from India, 12 from Canada and 14 from USA, while only one response was received from Benin, Russia and the Congo. In turn, the results from these countries were not very comparable. Thus, I only presented comparisons between larger geographical regions (Africa, Asia, North America, South America, Europe and Oceania), instead of between individual countries.

18 The questionnaire gave eight scales of TEK change - dramatic gain, gain, slight gain, no change, slight loss, loss, dramatic loss and other. See Appendix 5 for examples. 
The second limitation is the source of information. Although the information from community members is more direct and firsthand, due to the financial constraint, I could not distribute the questionnaire to communities in person and had to rely on the internet as my main tool for surveying. The most accessible respondents via internet communication were researchers ${ }^{19}$. Applying the concepts from cultural anthropology here, much of the data collected through the questionnaire and its follow-up interviews were etic (outsiders' views) ${ }^{20}$, rather than emic (insiders' views) ${ }^{21}$. As Headland et al. (1990) note, social scientists have long debated whether their knowledge is objective or subjective. The objectivity of researchers' observation and opinions is a concern of this research. The information provided by the researchers and practitioners may contain their personal or professional bias and judgment, which may lead to some bias in the results.

Despite the above limitations, the results of this research are still valuable in terms of an initial attempt to create globally applicable typologies for TEK threats and actions; in the future, more thorough surveys can further hone the classification. Ideally, this process could resemble the long, collective development of the IUCN classifications of threats to and actions for biodiversity, which involves multiple organisations and researchers, and has been tested over thousands of cases through its network (Salafsky et al., 2008). For future research, there are two urgent needs: 1) collecting more data from each country more evenly and thoroughly; and 2) comprehensively testing the typologies in various locations and cases.

There are also limitations to the case study. First, the cooperative activities (both on the research site and in wider China) have only been active for a short period of time. Their long term effects on recovering traditional institutions and wider aspects of TEK, as well as on community empowerment, need long-term monitoring and further research.

\footnotetext{
${ }^{19}$ Accounting for $74 \%$ of the total respondents.

${ }^{20}$ Etic is a description of a behaviour or belief by an observer, in terms that can be applied to other cultures (Morris et al., 1999).

21 Emic is a description of behaviour or a belief in terms meaningful (consciously or unconsciously) to the actor; that is, an emic account comes from a person within the culture (Morris et al., 1999).
} 
Second, the implementation of the Cooperative Law and assisting policies may vary in different locations. Local governments will play a critical role in determining the degree of legal enforcement. In turn, the law could lead to different cooperative development at the local level, and ultimately have different effects on TEK and its revitalisation. Lastly, TEK is not a homogeneous system of thought; TEK may vary enormously among different cultural groups and ecosystems. Thus, the conclusions from this research in Inner Mongolia are not necessarily applicable to other locations and other TEK systems. So far, this research has been the first to focus on the effects of the Cooperative Law on TEK and TEK conservation in Inner Mongolia and China. I hope it may inspire further research in this field and in wider areas of the world. 


\section{CHAPTER THREE}

\section{TEK THREATS}

In Chapter One, I discussed the status of TEK and threats to TEK in general. In this chapter, I will focus on a detailed analysis of the drivers of TEK degradation (which I refer to as TEK threats in this thesis), and present a typology of TEK threats developed from this research. The discussion will cover how to use the typology to understand the causal web of TEK degradation, how this understanding refers to the theoretical framework, and how the typology can be used by different actors.

\section{INTRODUCTION}

\subsection{Why this study?}

Since the 1970s, the discussion and research about TEK, with a special focus on the causes of TEK loss, has earned increasing attention from non-indigenous societies and academics. In the literature I have reviewed for this research (time ranged from 1976 to 2010), a great variety of drivers of TEK loss have been identified. Most of these studies focus on particular cause/s in particular locations ${ }^{22}$ (e.g. Benz et al., 2000; Case et al., 2005; Crystal, 2000; Godoy et al., 1998; Milton, 2001; Nettle and Romaine, 2000; Olsen and Helles, 1997; Raza, 2007; Ross, 2002; Wavey, 1993), while several studies focus on synthesising the causes of TEK loss into categories (Ellen, 2007; Lizarralde, 2001; Zarger and Stepp, 2004; Zent, 2001).

However, the level of the agreement on these causes or on categorising the threats is limited. The lack of a widely used threat classification system might be caused by differences in research attributes such as location, disciplinary perspectives, domains of TEK that the research focuses on, and different research methods. For example, while traditional language loss has been recognised as a key cause of TEK loss by a number of

\footnotetext{
$2289 \%$ of the literature I have reviewed $(\mathrm{n}=152)$ are empirical studies at local or regional levels.
} 
linguists and anthropologists (e.g. Crystal, 2000; Maffi, 2001, 2005; Zent and Maffi, 2009), market influence has been highlighted from a political economic perspective (e.g. Godoy et al., 1998, 2005; Reyes-Garcia et al., 2005a; Ross, 2002). This example reflects the fact that researchers from different disciplines approach issues from different perspectives and may focus on different domains of TEK. It is thus inevitable that they come up with findings and results that differ from each other.

Measuring TEK change has never been an easy task. There are several major challenges faced by TEK researchers. The first and most common challenge is the lack of baseline data. The academic research of TEK has only been carried out for several decades. The record of TEK prior to this period is missing from many locations around the world. This scarcity of baseline data creates a fundamental difficulty for any quantitative measurement and comparative study of TEK change (Zent, 2001). Thus, virtually all TEK research, including this study, is structured within a limited time-frame over the last few decades. This time frame may capture some significant TEK change (e.g. Tang and Gavin, 2010), but the relatively short research period and lack of baseline data means that researchers are sometimes unable to measure the change. Alternatively, many researchers measure the variation of TEK based on its social variables, such as age (Zent and Zent, 2004), gender (Begossi et al., 2002; Case et al., 2005), residence length (Nythus et al., 2003), involvement in formal education (Quinland and Quinland, 2007; Reyes-Garcia et al., 2005b), access to market (Geust, 2002; Godoy et al., 2005; ReyesGarcia et al., 2005a), and comparisons between different communities or regions (e.g. Ghimire et al., 2004; Reyes-Garcia et al., 2007a, 2007b). Putting all this research together may help to draw a clearer overall picture of TEK change.

The second challenge for examining TEK change over time is the lack of a standard methodology. As Reyes-Garcia et al. (2005a:21) point out, "researchers have used a large range of methods to collect data on traditional ecological knowledge, but few studies have replicated methods used in previous research or compared results across different methods... This situation exacerbates the difficulty of comparing studies". Let us take an example of data collection to see the variety of research methodology used in the field. 
For measuring the theoretical dimension of TEK (e.g. the knowledge of medicinal plants, the knowledge of canoe-building), researchers usually choose semi-structured interviews or structured questionnaires. However, questionnaires may include a wide variety of data-gathering mechanisms, including multiple-choice questions, pile-sorting, true-false questions, free-listing, and triad tasks (Reyes-Garcia et al., 2006c) (for the description of these methods, please see Bernard, 2010). For assessing practical dimensions of a TEK system (e.g. the skills of using medicinal plants, the skills of trail reading for hunting activities), data may be collected through researchers' direct observation, or through informants' self-reports. In addition, the sample size of different TEK studies varies enormously (e.g. seven in Geissler et al., 2002 to 622 in Nyhus et al., 2003). This lack of methodological uniformity inhibits comparability and is the main obstacle to a more simplified comprehension of the complex empirical reality of TEK threats (Zent and Maffi, 2009).

The third challenge is the multiple domains of TEK systems. As Berkes' (2008) TEK concept diagram indicates (see Chapter 1, Figure 1.1), TEK is a multi-level system which encompasses knowledge of local environment, animals and plants, resource management practices, social institutions, worldviews and beliefs. TEK research thus requires cross-disciplinary perspectives and mixed research methodologies. It is common that a researcher or a research team is able to access and study one or a limited number of TEK domains, but rarely, if ever, has TEK research undertaken a comprehensive whole system assessment. So far, most TEK research efforts have focused on ethnobotanical knowledge of indigenous societies, including medicinal plants (Benz et al., 2000; Geissler et al., 2002; Prince et al., 2001; Sternberg et al., 2001), wild food plants and crops (Boster, 1986; Godoy et al., 1998; Rocha, 2005; Zarger and Stepp, 2004; Zent, 2001), and other plants with significant economic values (Byg and Balslev, 2001; Hanazaki et al., 2000; Kristensen, 2003; Reyes-Garcia et al., 2005a, 2005b; Uma Shaanker et al., 2004). The wider layers of TEK, especially the social institutions, world view and beliefs of indigenous populations, have been studied to only a very limited degree (Reyes-Garcia et al., 2006). 
There is also a variety of terminology that researchers have used to describe similar or even identical phenomena (see an example under section 1.3). This variety of terminology can be troublesome for cross-project and cross-region communication. Valid communication and comparison of TEK threats requires a standard classification system. Such a system would enable comparable results to be produced and identify common patterns of TEK status despite the different disciplinary backgrounds and terminologies of researchers.

From the above discussion, we can see disagreements or disconnects about TEK threats from three aspects: 1) researchers often emphasise different threats due to their different research perspectives; 2) the methodologies of TEK threat identification are different; and 3) the terminologies used when describing the problems are different. These disagreements thus make the threats identified from different studies difficult to compare. The variety also makes cross-study communication and comparison difficult, which can inhibit the development of TEK understanding at a global scale, and the formation of a global network of TEK monitoring and conservation. As Zent (2001:191) points out, "Although some analysts have pointed out rather vaguely and generally to cultural and economic globalisation forces as the main reasons why TEK is disappearing at such an alarming rate, the precise determining factors, whether of local, regional, national, or international origin, and their complex interactions are still not well understood".

The above discussion has pointed out several gaps in the literature. By applying a global survey and comprehensive review of the global pattern of TEK threats, and developing a standard classification of TEK threats, this study aims to fill these critical research gaps.

\subsection{Terminology issues}

Several different terms have been used when referring to the decline and/or degraded status of TEK, or in other words the negative change of TEK status in the literature: TEK loss, TEK degradation, TEK erosion, and sometimes TEK change. The first three terms 
address a similar meaning, which refers to the decline and deteriorating condition of TEK. Yet TEK loss might place more emphasis on the quantitative decline of TEK, while degradation and erosion might place more emphasis on the qualitative deterioration of TEK. However, there is no strict and clear distinction between these terms, and they are used interchangeably in the literature. TEK change, on the other hand, may not always address the deterioration of TEK; it may also indicate an increase of TEK either qualitatively or quantitatively, or simply a shift due to the change of the ecosystem which an indigenous group lives on (McCarter, 2012). In this thesis, both TEK degradation and TEK loss are used when referring to a negative change of TEK status.

During the questionnaire design, I considered the objectivity of the terminology, and developed questions carefully with a neutral tone. For example, question five of the questionnaire reads as "Based on your experience, how would you assess the general change of TEK in your location over the last 50 years"? The options were given with eight scales, ranging from dramatic loss to dramatic gain (see more details in Appendix 5). Then question six follows as "In your opinion, what are the main reasons for TEK change in your location"? By using TEK change and giving full scales of change, the questionnaire avoided the potential implication to the respondents that it merely focused on collecting data about negative changes to TEK.

\subsection{Why is a typology important?}

As I mentioned above, a standard classification system of TEK provides a tool for TEK holders, scientists and practitioners to identify problems and solutions, and enables them to communicate across projects (Salafsky et al., 2008). For example, if one community addresses the main threat to TEK as "indigenous language loss", the neighbouring community recognises their TEK threat as the "loss of elders", and another community in the same area consider that "young people are moving away" is the main threat to their TEK, it sounds like they are talking about different issues. However these causes may all relate to a direct threat of TEK - loss of transmission pathways. This example illustrates how a standard classification system can be used as a diagnostic tool 
for identifying TEK threats efficiently. TEK holders, TEK conservation practitioners and scientists can use the typology to identify and describe what is happening in their site. They can also scan through the threat classification and see if they recognise any threats that have been ignored in their own analysis of the situation. Once the threats are identified, in association with the use of conservation action typology, conservation actors can identify and prioritise actions to deal with the threats.

At larger scales, a standard classification provides a common language for community members, practitioners and scientists to communicate and exchange their experience. This is extremely important since we are now living in a globalised world (Berkes, 2007). Sharing and exchanging lessons and experience can effectively save researchers time and effort by avoiding unnecessary repeated experiments. By using a common language, information transmission and sharing across regions and across organisations becomes possible (Zent, 2001). A unified system also offers the possibility and likelihood of building common databases of TEK threats and TEK conservation. By searching through a common database of TEK conservation projects, TEK holders and practitioners may find other projects that face similar threats, and find actions that have been effective in such situations. To date, TEK databases exist in several countries, but most of these databases are at national or regional scales. There is no unified international database that draws overall pictures of TEK and TEK conservation globally. Similarly, as addressed by Salafsky et al. (2008:2) in the field of biodiversity conservation, "[a standard lexicon] would enable conservationists around the world to share and exchange experiences through common databases of conservation practice, thus facilitating cross-project learning and the development of principles about what actions are effective under different conditions to encounter different threats".

Maybe even more importantly, a unified classification system provides a powerful tool for identifying the global pattern of TEK threats to inform conservation actions. This pattern will be useful for guiding national and international decision making and funding allocation. International conventions and agreements have played an increasingly important role in managing key human commons, including natural 
resources and cultural heritage in recent decades (Maffi, 2005). For example, the Convention of Biological Diversity has played a significant role in guiding conservation policies at a national level around the globe, as well as leading international cooperation on issues of biodiversity conservation. The IUCN-CMP (World Conservation Union Conservation Measure Partnership) Classification of Threats to Biodiversity provides an salient example of how unified classifications are useful and essential to the success of conservation efforts through a global network (Salafsky et al, 2008, 2009). As Salafsky et al. (2008) point out, the biodiversity conservation classification system improves information sharing and learning among conservation practitioners within and across organisations. It ultimately contributes to the creation of a systematic science of biodiversity conservation. There are strong links and similarities between the conservation of biological and cultural diversity. As summarised by Maffi (2001:3), "Over the past decades, scholars and advocates from a variety of fields have been increasingly pointing to the detrimental effects of current global socioeconomic and environmental processes on the very objects of their concern: biological species, the world's ecosystems, human cultures, linguistic groups and their traditional knowledge... these various manifestations of life on Earth are facing comparable threats of radical diversity loss". The development of the TEK typologies has been deeply inspired by the IUCN-CMP's Classifications of Threats and Actions to Biodiversity created by Salafsky and his colleagues over the last decade (Salafsky et al., 2002, 2003, 2008, 2009). Also inspired by the IUCN-CMP model, I suggest the following key concepts to illustrate the general components of any field situation in TEK research and conservation.

TEK Conservation Target is what a conservation action is trying to conserve. In this research, the conservation target is the entity of TEK, which includes knowledge of landscape and seascape, plants and animals; resource use and management practice; social institutions and worldviews and beliefs.

TEK Degradation is the decline and/or degraded condition of any or all aspects of TEK (e.g. traditional knowledge decline; deterioration in traditional resource management and livelihood practices). 
Direct Threats are the human activities that directly cause the destruction, decline and/or degradation of TEK conservation targets (e.g. the loss of pathways of TEK transmission; religious conversion).

Underlying Threats are the causal factors of direct threats, which usually include cultural, economic, political and institutional factors (e.g. government policy and legislation; influence of outside markets).

Conservation Actions are the actions undertaken by TEK holders and/or practitioners to conserve and revitalise TEK, and to empower TEK holders (e.g. community cultural camps; customary schools; TEK documentation). TEK conservation actions are a direct response to TEK degradation, where actions may be either reactive or proactive.

By applying these concepts to the analysis of real-world situations, a model was developed, as shown below:

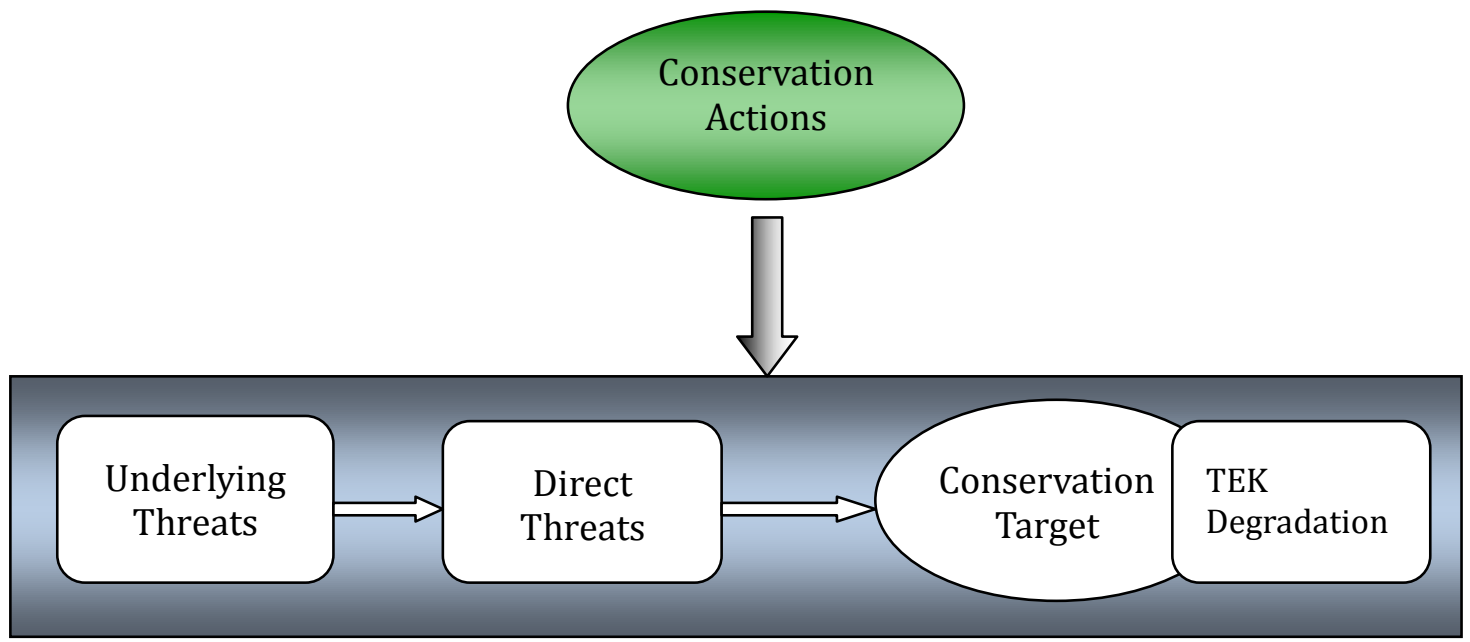

Figure 3.1 A model of a TEK conservation project (inspired by Salafsky et al., 2008)

In real-world cases, any single TEK threat does not act alone. Rather, TEK degradation is usually the result of multiple threats occurring simultaneously, and as a result of 
historical and cumulative processes. Usually one direct threat is caused by several underlying threats, and these underlying threats may have other underlying causes. The ultimate goal of this study is to encourage effective TEK conservation by providing a standard lexicon for problem identification and a toolbox for conservation actions. As Figure 3.1 shows, conservation actions can be employed to underlying threats, direct threats, or directly to the conservation target. This creates great flexibility for conservation actors when scoping targets and designing actions. More details of action design will be discussed in the next chapter.

\subsection{Aim and objectives}

This chapter aims to investigate the global pattern of TEK change, to explore the threats that TEK faces at the global scale, and to develop a typology of TEK threats.

The research objectives then fall into five themes:

1. Exploring research gaps by reviewing TEK related literature with a focus on current knowledge about TEK change and the causes of TEK loss.

2. Collecting data about TEK status and the causes of TEK loss by carrying out a global survey.

3. Based on the survey results, to construct an overview of TEK status globally, and then compare the survey results with the literature.

4. Combining the results from the literature review and survey to produce a typology of TEK threats.

5. Using the typology to investigate the causal web among different TEK threats.

\section{RESULTS}

The research results are generated from two major methods: 1) the literature review provides a comprehensive understanding of TEK and TEK research with an emphasis on the causes of TEK loss addressed by scholars. It creates a foundation for questionnaire 
design and provides baseline data for developing a TEK threat typology; and 2) the survey and its follow-up interviews identifies patterns of TEK change at a global scale, and explores the threats TEK faces (see more details of research methods in Chapter 2, section $3.1,4.1 \& 5)$.

\subsection{Geographical pattern of TEK studies}

\subsubsection{Geographical distribution of survey responses}

The survey responses ${ }^{23}$ cover 48 countries and regions. Among 137 effective responses, $24 \%$ of them are from Asia, including Cambodia, China, India, Indonesia, Malaysia, Laos, Mongolia, Nepal, Pakistan, Thailand and Vietnam; 19\% of them are from Africa, including Benin, Cameroon, Congo, Cyprus, Kenya, Morocco, Mozambique, South Africa, Tanzania and Uganda; 17\% are from North America, including Canada and United States of America; $15 \%$ are from Oceania, including Australia, Marshall Island, New Zealand, Solomon Island and Vanuatu; 14\% are from Europe, including Belgium, France, Italy, Norway, Macedonia, Portugal, Russia, Serbia, Spain, Switzerland, UK and Western Balkan region; and 11\% are from South America, including Argentina, Bolivia, Colombia, Ecuador, Mexico, Panama, Peru and Venezuela (see Figure 3.2 below).

\footnotetext{
${ }^{23}$ Please note there is distinction between geographic distribution of survey responses and of survey respondents. The former one represents where the research has been carried out; the latter one indicates where the informants are based.
} 


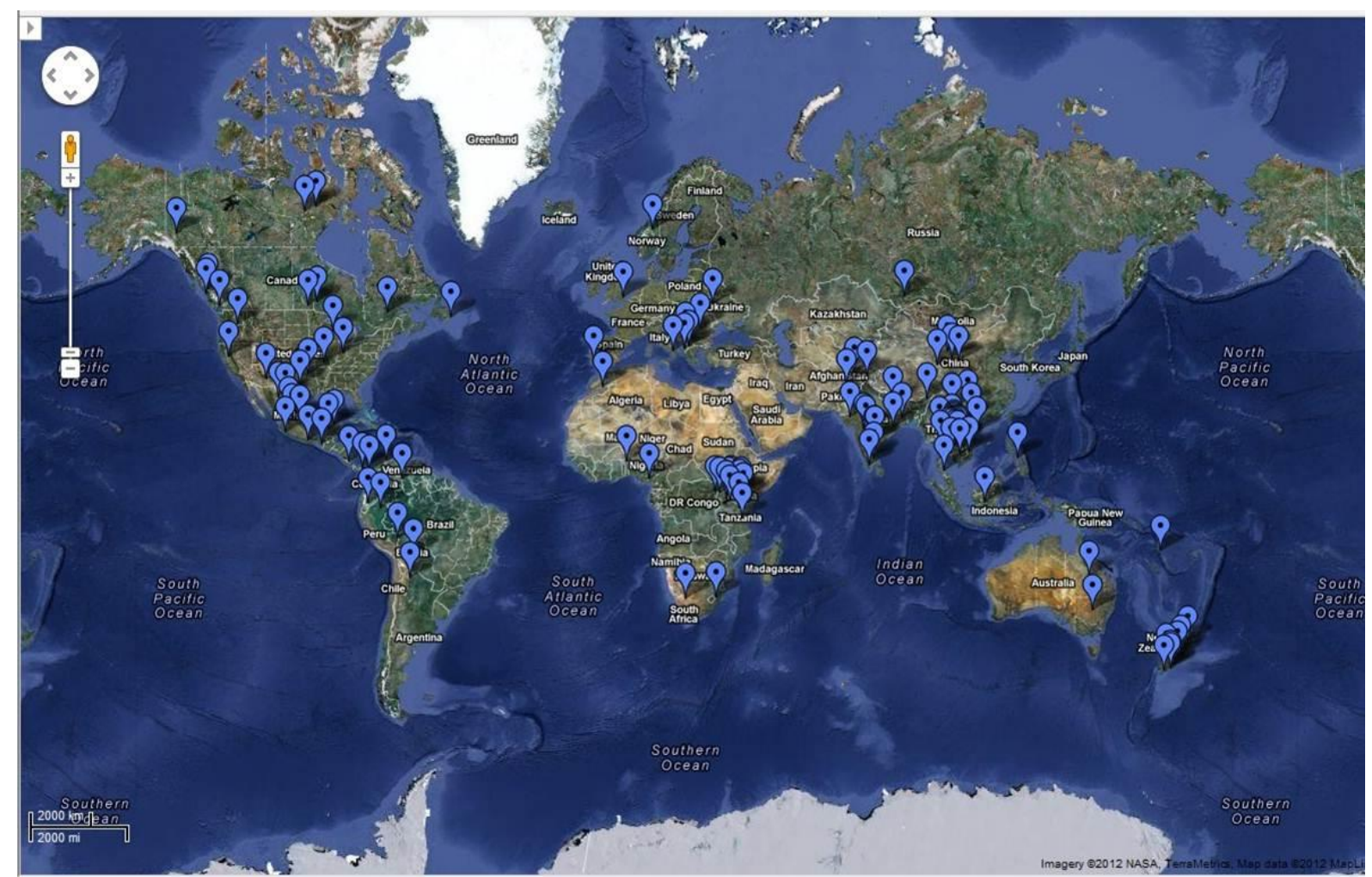

Figure 3.2 Map of the survey responses' geographic distribution.

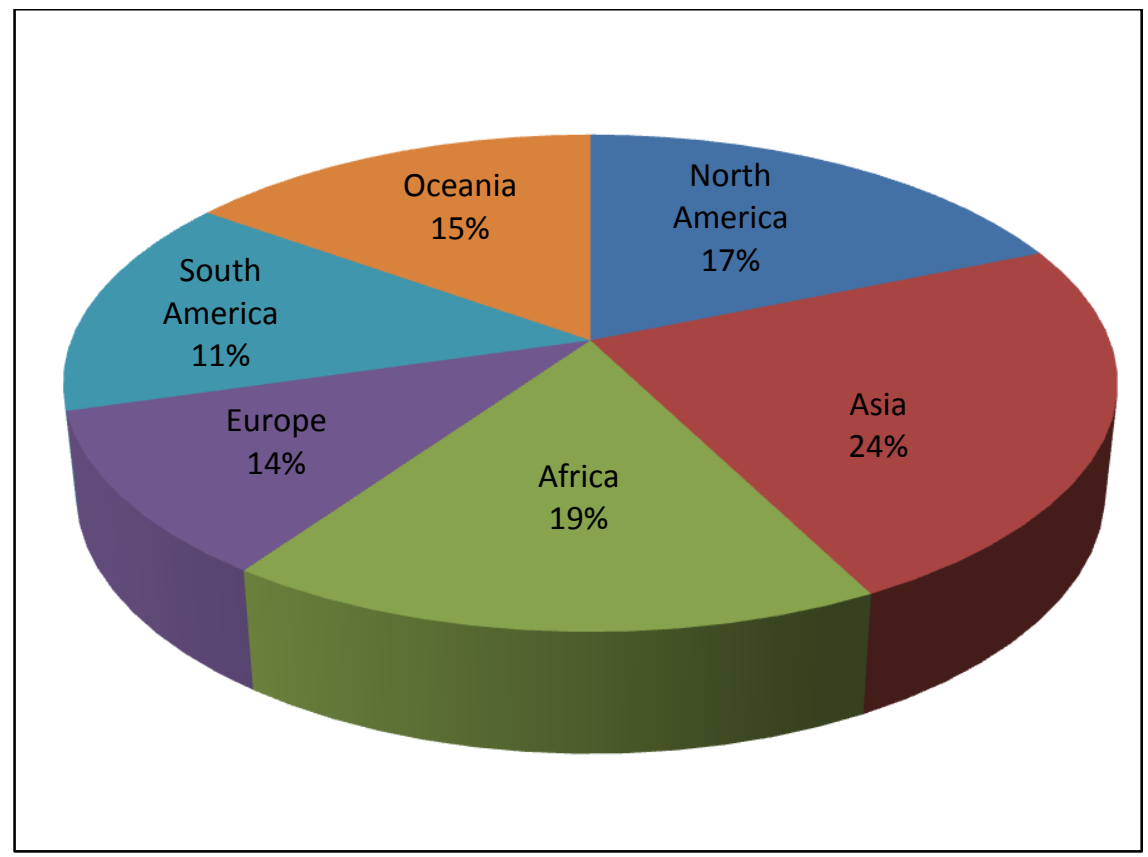

Figure 3.3 Geographical distribution of survey responses $(n=137)$ 
From the figure above, we can see a relatively even pattern of geographical distribution of the survey responses. The survey responses covered six major continents without significant concentration on any area.

\subsubsection{Geographical pattern found in the literature}

Among 152 studies I reviewed (time ranged from 1976 to 2010), 64\% (n=97)

concentrate on South America. The rest of the literature is shared between Africa (15\%, $\mathrm{n}=23)$, North America (11\%, $\mathrm{n}=17)$, Asia (9\%, n=11, mainly concentrate on Southeast Asia) and Oceania (2\%, n=3) (see Figure 3.4) 24 .

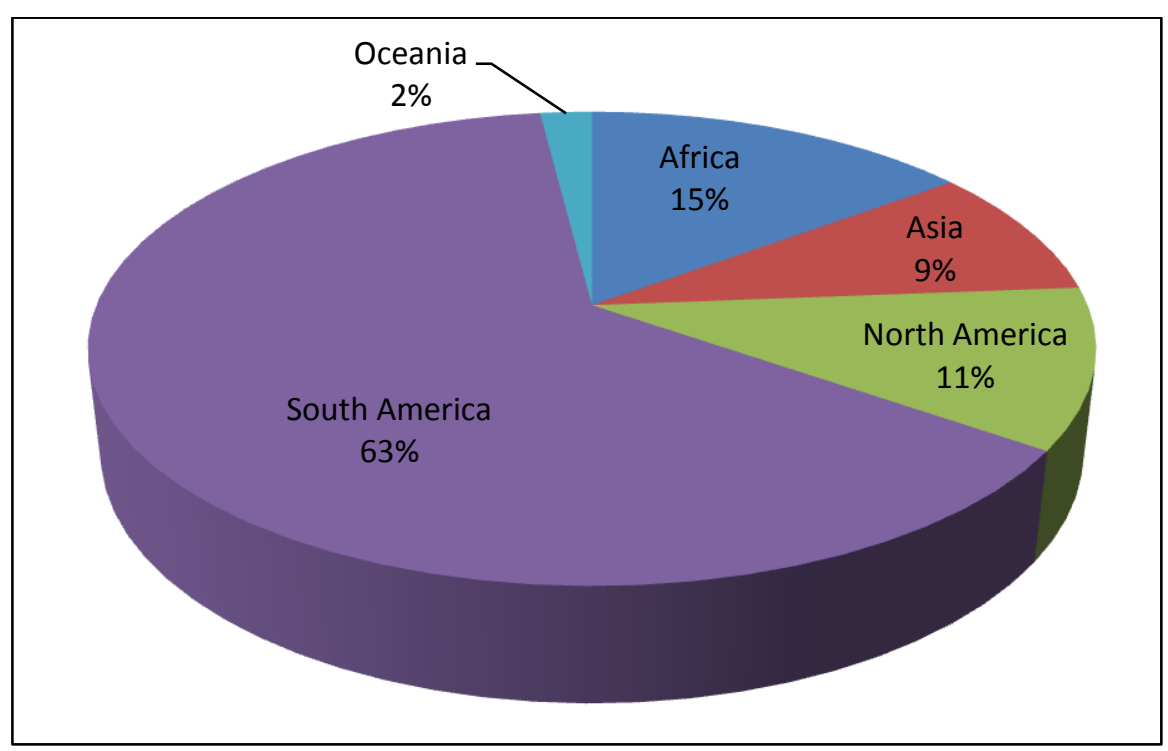

Figure 3.4 Geographical pattern found in the literature $(n=152)$

A similar pattern is also addressed by Reyes-Garcia et al. (2005). They find that $64 \%$ of the studies they reviewed $(n=46)$ took place in Latin America, 17\% in Africa, 9\% in Asia, and the remaining studies took place in other regions of the world. No study of traditional ecological knowledge with European populations was found in either literature review.

\footnotetext{
${ }^{24}$ Please note that, as this research mainly focuses on English-language articles, the result may reflect geographic bias of academics.
} 
This uneven geographical pattern indicates a literature gap in TEK research. For instance, the vast area of Asia, apart from south-east Asia, is almost a blank spot for research. Among 152 studies, I only found three studies from China (Wu et al., 2001; Xu and Melick, 2007; Xu et al., 2008), two studies from Mongolia (Fernandez-Gimenez, 2000, 2002), two from Nepal (Ghimire et al., 2004; Olsen and Helles, 1997) and one from Bangladesh (Brush, 2005). The rest of Asia requires much more research attention and effort. Likewise, many regions in Africa and Oceania (e.g. various islands) possess rich TEK, but are not yet addressed in the literature with proper weight. Even though Europe has been considered to have the least remaining TEK, it is still worth the research effort to explore its TEK status as part of a global pattern.

Comparing the results from the survey and literature review, the survey collected data from Asia and Oceania that supplement their low study rate in the literature; it also collected important data from Europe which is missing from the literature.

\subsection{The trend of TEK change}

\subsubsection{Trend of TEK change claimed by survey responses}

The survey results show that, $87 \%(\mathrm{n}=119)$ of the responses claimed a trend of TEK loss in their locations, while $2 \%(n=3)$ stated that there was no change of TEK, and $7 \%(n=10)$ claimed a gain of TEK. Six informants (4\%) chose other, they indicated that either they didn't know or it was too complicated to say. It is worth mentioning that, due to the lack of standard procedure of measuring TEK, it is difficult to obtain a uniform assessment of TEK change. The scales used to describe TEK status in the questionnaire are subjective perceptions of the respondents. 


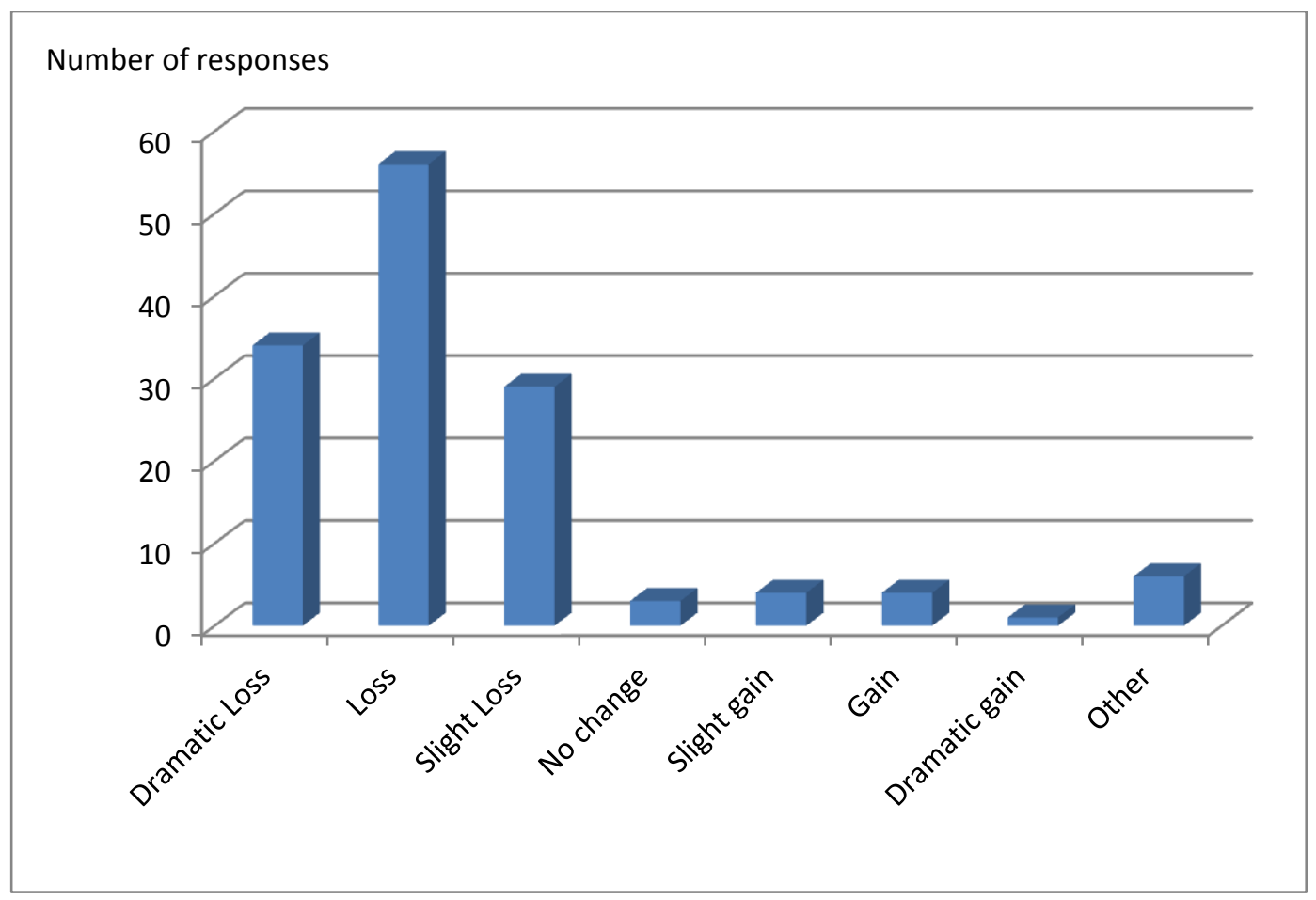

Figure 3.5 The overall trend of TEK change - Results of the survey $(n=137)$

To further define the pattern of TEK change, I also examined the links between TEK change and geographical regions. The result shows that regardless of geographical divisions, responses pointed to some degree of TEK loss ( $>70 \%$ of the responses in every geo-region who answered dramatic loss, loss or slight loss). Increases in TEK were claimed in only a limited number of cases $(n=9$, four from Europe, three from North America and two from Oceania). Only three (two from Africa and one from Europe) claimed no change in TEK status in their locations.

\subsubsection{Trend of TEK change identified in the literature}

I attempted to carry out a similar assessment of TEK status as identified in the literature. However, due to the lack of a standard set of scales (e.g. used in the questionnaire), and the large variety of the language used to describe TEK status in the literature made it very difficult to make such an analysis. I thus could only identify a general pattern by distinguishing between loss, no change, gain and no mention (see Figure 3.6 below). 


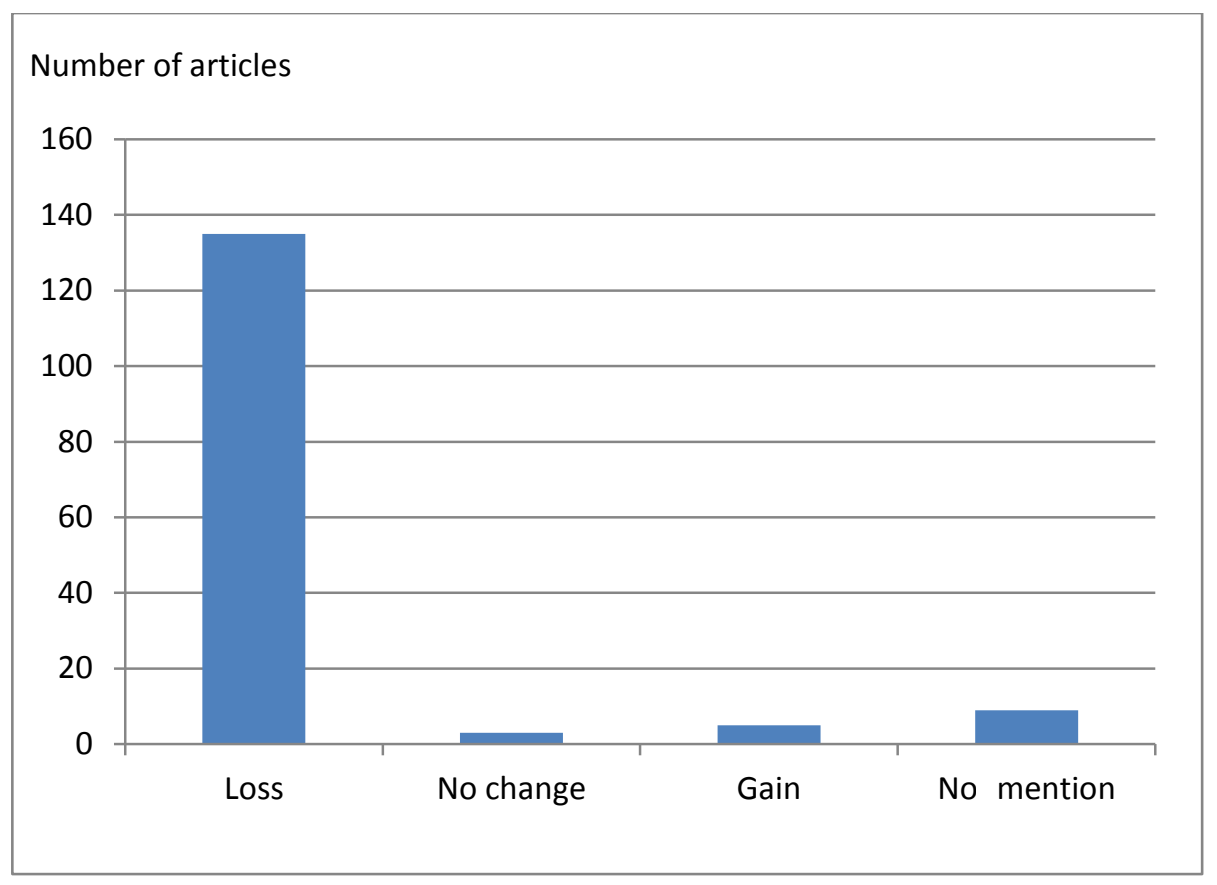

Figure 3.6 The overall trend of TEK change -Results of the literature review $(n=152)$

As the above figure illustrates, among all 152 TEK studies, 135 (89\%) reported a loss of TEK, while three (2\%) claimed no change, and five (3\%) noted a gain of TEK in the research locations. Beside these trends, nine studies (6\%) did not mention or discuss the status of TEK. The overall trends of TEK change explored from the survey and the literature present a similar pattern: the majority claimed loss of TEK alongside a minor rate of no change or a gain of TEK.

\subsection{The typology of TEK threats}

The typology of TEK threats presented in this section is a product of the triangulating results from the survey, its follow-up interviews, literature review and my own case study. It has also been reviewed and tested by seventeen key informants including eight community members and nine experts from different fields (see more details in Chapter 2 , section 4.1). The typology has been modified according to these typology reviewers' feedback. 


\subsubsection{Direct threats to TEK}

As discussed in section 1.3, direct threats refer to the human activities that directly cause the destruction, decline and/or degradation of any or all aspects of a TEK system. In this classification, the direct threats are classified into six categories, covering TEK transmission, traditional livelihood practices, traditional religion and beliefs, indigenous environment and natural resources, traditional rights and traditional institutions. Each category is further divided into several sub-categories, which define the category more specifically. Both levels of the classification are comprehensive, including all possible threats noted in the survey and in the literature. In this typology, definitions of each category and examples of each sub-category are given. Detailed discussion with examples from both survey responses and literature review will be presented under the table of the typology. 


\section{Table 3.1 Typology of Direct Threats to Traditional Ecological Knowledge}

\begin{tabular}{|c|c|}
\hline Threats by categories & $\begin{array}{l}\text { Definitions for each category \& } \\
\text { Examples for each sub-category }\end{array}$ \\
\hline 1. Loss of pathways of TEK transmission & $\begin{array}{l}\text { Complete or partial loss of the TEK transmission processes } \\
\text { between individuals and generations, within a community } \\
\text { and/or across communities }\end{array}$ \\
\hline 1.1 Loss of traditional language & $\begin{array}{l}\text { Enforced use of coloniser's language; } \\
\text { Elders passing away without transmitting the indigenous } \\
\text { language to younger generations }\end{array}$ \\
\hline $\begin{array}{l}1.2 \text { Influence induced by formal } \\
\text { educational system }\end{array}$ & $\begin{array}{l}\text { Suppression of traditional cultural expression in formal schools; } \\
\text { Devaluation and exclusion of TEK in curriculum; } \\
\text { Children learn knowledge from school in abstract, but not } \\
\text { "learning by doing" from their own community }\end{array}$ \\
\hline $\begin{array}{l}1.3 \text { Younger generations' absence from the } \\
\text { traditional community }\end{array}$ & $\begin{array}{l}\text { Younger generations resident in town for jobs or education and } \\
\text { disconnected from their own communities }\end{array}$ \\
\hline $\begin{array}{l}\text { 1.4 Influence induced by dominant } \\
\text { societies \& reduced incentive and interest } \\
\text { in TEK }\end{array}$ & $\begin{array}{l}\text { Increased amount of information and knowledge gained from TV; } \\
\text { Younger generations are more interested in the skills that enable } \\
\text { them to find a job in town }\end{array}$ \\
\hline $\begin{array}{l}\text { 2. Change of traditional livelihood } \\
\text { practices }\end{array}$ & $\begin{array}{l}\text { Reduced needs and practices of, or abandonment of } \\
\text { traditional livelihood, which often involves a transition } \\
\text { from traditional subsistence livelihood to non-traditional } \\
\text { livelihood activities, such as engaging with industrial and } \\
\text { trading activities }\end{array}$ \\
\hline $\begin{array}{l}2.1 \text { Reduced land-based/ sea-based } \\
\text { activities }\end{array}$ & $\begin{array}{l}\text { Engaging with trading activities to earn cash rather than } \\
\text { traditional crop plantation; } \\
\text { Working for factories instead of fishing }\end{array}$ \\
\hline $\begin{array}{l}2.2 \text { Reliance on modern products and/or } \\
\text { technologies }\end{array}$ & $\begin{array}{l}\text { Prefer or need to buy processed food from stores rather than } \\
\text { hunting or fishing for food; } \\
\text { Rely on compass during fishing trips instead of traditional } \\
\text { navigation skills }\end{array}$ \\
\hline $\begin{array}{l}2.3 \text { Use of Westernised primary } \\
\text { production systems }\end{array}$ & $\begin{array}{l}\text { Western style agricultural system - cattle ranching and privatised } \\
\text { land; } \\
\text { Replace traditional crops with introduced high yielding crops }\end{array}$ \\
\hline $\begin{array}{l}\text { 3. Change of traditional religion and } \\
\text { beliefs }\end{array}$ & $\begin{array}{l}\text { Loss or change of traditional religion and beliefs in the } \\
\text { importance of nature and local environment }\end{array}$ \\
\hline
\end{tabular}




\begin{tabular}{|c|c|}
\hline $\begin{array}{l}3.1 \text { Conversion to other dominant } \\
\text { religions }\end{array}$ & Conversion to Christianity or Islam \\
\hline 3.2 Loss of traditional beliefs & Younger generations do not believe in Holy Mountains any more \\
\hline $\begin{array}{l}\text { 4. Change of environment and natural } \\
\text { resources }\end{array}$ & $\begin{array}{l}\text { Change of physical environment due to relocation, and/or } \\
\text { degradation or depletion of natural resources }\end{array}$ \\
\hline $\begin{array}{l}\text { 4.1 Shift of physical location and } \\
\text { environment }\end{array}$ & $\begin{array}{l}\text { Enforced relocation of indigenous groups; } \\
\text { Indigenous people emigrate to urban areas }\end{array}$ \\
\hline 4.2 Resource degradation & $\begin{array}{l}\text { Desertification, grassland and forest degradation; } \\
\text { Loss of key species which carry cultural identity }\end{array}$ \\
\hline 5. Loss of traditional rights & $\begin{array}{l}\text { Complete or partial loss of traditional land, loss of resource } \\
\text { or resource rights, and loss of traditional access to natural } \\
\text { resources }\end{array}$ \\
\hline $\begin{array}{l}\text { 5.1 Loss of traditional land and/or land } \\
\text { tenure }\end{array}$ & $\begin{array}{l}\text { Enforced removal of indigenous people from their traditional land } \\
\text { by coloniser; } \\
\text { State government claims the ownership over indigenous land }\end{array}$ \\
\hline 5.2 Loss of traditional land use & Urban expansion occupies indigenous farm land \\
\hline 5.3 Loss of access to traditional resources & $\begin{array}{l}\text { Reserve set aside inside of indigenous land, and banning of the } \\
\text { traditional use of bush meat }\end{array}$ \\
\hline 6. Loss of traditional institutions & $\begin{array}{l}\text { Complete or partial loss, or change in traditional social } \\
\text { organisations, customary norms and traditional rules of } \\
\text { resource use and management }\end{array}$ \\
\hline $\begin{array}{l}\text { 6.1 Replacement of traditional resource } \\
\text { governance }\end{array}$ & $\begin{array}{l}\text { The government-appointed community leaders replace } \\
\text { traditional community institutions }\end{array}$ \\
\hline $\begin{array}{l}6.2 \text { Loss of other aspects of traditional } \\
\text { institutions }\end{array}$ & $\begin{array}{l}\text { Loss of the traditional way of resolving conflict regarding } \\
\text { resource use and sharing; } \\
\text { Loss of traditional financial institutions, e.g. the use of national or } \\
\text { regional currency leads to the loss of the traditional mechanisms } \\
\text { of exchange across communities; } \\
\text { Loss of traditional rituals, ceremonies and other cultural and } \\
\text { spiritual practices }\end{array}$ \\
\hline
\end{tabular}

Below, I will provide an analysis of each category with examples from the survey and the literature. 


\subsubsection{Direct threat one - Loss of pathways of TEK transmission}

As addressed in the typology, loss of pathways of TEK transmission refers to complete or partial loss of the TEK transmission processes between individuals and generations, within a community and/or across communities. The study of TEK transmission has been initiated by anthropologists within the frame of cultural transmission, which is defined as "the process of acquisition of behaviours, attitudes, or technologies through imprinting, conditioning, imitation, active teaching and learning, or combinations of these" (Cavalli-Sforza et al., 1982:19). The cultural transmission usually takes place in two directions: 1) vertical transmission which happens between parents and children (Cavalli-Sforza et al., 1982; Eyssartier et al. 2008; Hewlett and Cavalli-Sforza, 1986). The natural discrete time unit of this kind of transmission is the generation; and 2) horizontal transmission, which happens between peers of the same generation (Aunger 2000; Guglielmino et al., 1995; Lancy 1996; Rogoff 1981). This category focuses on the loss or decline of the pathways in which TEK is transmitted, including traditional languages, traditional educational practices, and traditional knowledge generation and exchange systems.

Many scholars from various fields (e.g. anthropology, linguistics, biocultural conservation and ethno-sciences) recognise that indigenous language is a key indicator of TEK and cultural diversity, and the loss of language diversity directly affects the loss of knowledge diversity (Crystal 2000; Harrison, 2007; Maffi, 2001, 2005; Nettle and Romaine 2000, 2002; Zent and Maffi, 2009). Indigenous languages are the carriers of traditional knowledge and practical skills within indigenous groups. In most cases, the meaning of indigenous concepts and perceptions are hard to translate to other languages (Crystal, 2000; Harrison, 2007). A large number of studies claim the loss or degradation of indigenous languages worldwide. For example, according to Lizarralde (2001), 33\% of the indigenous languages $(n=135)$ are lost in South America compared with its pre-European period. Within those remaining languages $(n=329)$, only 146 are spoken by 1000 or more speakers. As Ladefoged (1992: 810) points out, "[a language] with less than 1000 speakers ... is not likely to remain a distinct entity". A similar pattern at a global scale is discovered by Harrison (2007). According to Harrison's estimate, only 
half of 6,912 distinct human languages that were spoken in 2001 may still be spoken by the end of this century. Nettle and Romaine (2002) echo this statement in their book Vanishing Voice: The Extinction of World's Languages. As many linguists warn, the critical knowledge carried by these languages will also go extinct by the end of the $21^{\text {st }}$ century (e.g. Harrison, 2007; Krauss et al., 2004; Maffi, 2001, 2005).

The loss of traditional languages was also identified as a major cause of TEK loss by survey respondents, including multiple community members $(\mathrm{n}=11)$ and researchers $(n=21)$. For example, a community leader from Cibecue (Dishchii bikoh) in Arizona of USA stated, "Our language and traditional practices are closely tied to the land, in many ways it is used in describing objects, teaching moral lessons and expressing our purpose on this land. Since the loss of our traditional language and our forest, our traditional ecological knowledge has become more and more threatened". A number of researchers observed the trend that a significant proportion of young indigenous people are not able to speak their native dialect, thus they are not able to communicate with native elders (e.g. a researcher from Korup area of Cameroon). Another critical trend is that many native elders who were raised with traditional practices have died without passing their language, knowledge and skills to the younger generations (perceived by a researcher from Sahaptin, Columbia Plateau, in Pacific Northwest of USA, 2010). The loss of languages and loss of elders indicate the direct loss of traditional knowledge that is embedded in traditional languages.

Traditional educational practices are another major mechanism of cultural and TEK transmission. However, a large number of studies claim that the introduction and implementation (often by enforcement) of formal education ${ }^{25}$ to indigenous populations has negatively influenced TEK acquisition (e.g. Benz et al, 2000; Cruz García 2006; Grigorenko et al. 2004; Quinlan and Quinlan, 2007; Rocha, 2005; Sternberg et al. 2001; Voeks and Leony 2004; Wester and Yongvanit 1995; Zent 1999, 2001). When indigenous

\footnotetext{
25 The structured educational system that is usually state-supported and state-operated. It is usually considered as the opposite term to learning-by-doing, informal education, which is a basic character of traditional educational practices.
} 
children spend time in formal schools ${ }^{26}$, they are often detracted from learning subsistence and other traditional activities in their own communities (Cruz Garcia, 2006; Stenberg et al, 2001). According to Nabhan (1998), the Tohono O'odham children of the south-western USA can only name a small fraction of the common plant and animal species in their local environment when compared with their grandparents. In many areas of USA and Canada, it is common that indigenous American Indian children have to attend residential schools, in which native languages and traditional cultural expression are banned or discouraged (Zent, 2001). Instead, the schools educate children that traditional lifestyle is backward and should be replaced with "civilised" ones. As a result, many children devalue their traditional knowledge and culture, and prefer to choose Western lifestyle when they grow up (Inglis, 1993; Wavey, 1993).

It is also worth noting that the formal school system commonly teaches in national or regional languages (or so-called formal languages), usually non-indigenous languages. Indigenous children are more and more exposed to learning and speaking formal languages along with the wide spread formal educational model. The increased use of formal languages has been reported to negatively impact acquiring and maintaining TEK (e.g. Benz et al., 2000; Reyes-Garcia et al., 2005; Zent, 1999, 2001). For instance, Benz et al. (2000) carried out a comparative study of the knowledge about plant use amongst indigenous groups in Mexico. The results show that the knowledge is more diverse and evenly shared by the Huastec people who still speak an indigenous language, compared with Spanish-speaking indigenous groups. Reyes-Garcia et al. (2005b) also found that the use of national language has negatively affected ethnobotanical knowledge among the Piaroa people in Venezuela and Tsimane people in Bolivia. Linguistic development is a long-term process, and TEK that is carried by the language will not disappear overnight. We might find many examples where indigenous peoples with bilingual ability are still able to carry traditional knowledge and practices. However, as a longterm effect, increased ability in non-indigenous language competes with the use and

\footnotetext{
26 Please note that not all TEK is informally transmitted, some indigenous societies are still able to control their educational system in a traditional way (e.g. McCarter, 2012).
} 
acquisition of indigenous language and knowledge.

Echoing the finding in the literature review, the influence of formal education was addressed as a major cause of TEK loss by $42 \%$ of the survey responses. A significant number of researchers emphasised their observations of the negative impacts of formal education on traditional knowledge systems. For instance, a researcher who works in Campeche, Mexico noted that, "The Mexican state authorities work very hard to keep children in schools, which people interpret locally to mean these young people will be trained for something other than farming. Thus, young people are acquiring formal, academic knowledge that is conceptualised as competitively superior to TEK". A similar observation was made by a researcher who has worked in thirteen villages of two provinces (Panama and Darien provinces) in Panama. He described that, "In school, children learn to value different types of knowledge. For example, they are taught that successful people are teachers, doctors, and lawyers - not local leaders, farmers, fishermen or healers." In New Zealand, a researcher pointed out that, "formal schooling does not include TEK or a Māori27 ${ }^{27}$ orld view, so it tends to displace TEK. Meanwhile, younger people are not being taught the tikanga (lore) and mātauranga (TEK) by older family members". All these examples illustrate that the current model of formal education are threatening the transmission of TEK in many parts of the world.

Younger generations' absence from the community has been observed in both the literature and the survey. The absence of young indigenous people from their communities indicates their missing chance to learn and practice traditional knowledge and skills, and furthermore, to develop their pride in traditional knowledge and cultural identity. As a researcher from the Korup area of Cameroon noted, "younger people are staying in towns for education and coming to villages just occasionally. As a result, they are missing the skills to speak their dialect and to learn what elders can do!" A community elder from Alashan region, Inner Mongolia of China also pointed out that, "young [Mongolian] people stay in town for school and job nowadays. Very few of them are willing to come back and carry on a traditional pastoralist lifestyle". Driven by a

\footnotetext{
27 Maori is the indigenous group in New Zealand.
} 
larger force, more likely globalisation processes, there is also a corresponding movement of young indigenous people from developing countries to other countries, mostly developed Western countries, such as Britain, Australia, New Zealand and USA (perceived by a researcher from Paphos district in Cyprus).

\section{Although the influence induced by dominant societies and the reduced interest in}

TEK was only claimed by $6 \%$ of the survey responses, it helps explain why so many indigenous people have turned away from their traditional knowledge systems (Zent, 2001). As the survey results show, the major contributors to a dominant society's influences may include migration processes (either within a country or outward), contact with other cultural groups, involvement in market activities and governmental policy. For example, as an indigenous community member in Chitwan District of Nepal described, "Invasion of cultures and knowledge by other dominant groups is the main reason for TEK loss in our region". Similarly, a researcher from Arusha, Tanzania stated, "There is an increased mixture and interdependence of people from different ethnicities or even races who place different values and knowledge on the ecology of the place". These claims reflect the significant influences induced by dominant cultural groups on reducing the chance of TEK transmission.

In the literature, many studies also point out that contact with and influence from dominant cultures have changed indigenous cultures and value systems, and ultimately led to a reduced interest in traditional culture and practices by indigenous youth (e.g. Benz et al., 2000; Caniago and Siebert, 1998; Case et al., 2005; Cruz García, 2006; Hill, 2004; Lizarralde, 2001; Reyes-Garcia et al., 2007; Robert et al., 2004; Ross, 2002a; Voeks and Nyawa 2001; Zent, 1999; Zent and Maffi, 2009). For example, in the Western Ghats of India, due to the availability of Western goods, many indigenous families expressed shame at being seen by other people collecting wild food plants, even though they recognise these foods to be healthy and nutritious (Cruz García, 2006). As a result, the knowledge and use of wild food plants is declining. 


\subsubsection{Direct threat two - Change of traditional livelihood practices}

The change, in some cases the abandonment, of traditional livelihood practices has been claimed as one of the most fundamental driving factors of TEK loss worldwide, from both the literature (e.g. Ellen, 2007; Iskandar, 2007; Lizarralde, 1997, 2001; Raza, 2007; Soemarwoto, 2007) and the survey. This category refers to the reduced needs and practices, or abandonment of traditional livelihood. It often involves a transition from traditional subsistence livelihood to non-traditional livelihood activities, such as engaging in industrial and trading activities. As Berkes (2008) addresses, the best way to conserve a knowledge system is to put it to active use and practice. By applying the knowledge in livelihood and subsistence practices, one has the best chance to keep the knowledge system alive, and to appreciate its values (Inglis, 1993).

Much of the literature emphasises the influence of the market economy on changing traditional livelihoods (e.g. Godoy et al., 1998, 2005; Olsen and Helles, 1997; ReyesGarcia et al., 2005; Ross, 2002; Schultes, 1992). Markets bring both commodities that may not be traditionally available to a local group, as well as cultural contact with nonindigenous groups. In many cases, the availability of Western goods promotes a shift from traditional subsistence practices to a non-traditional lifestyle (Guest, 2002; ReyesGarcia, 2007). Godoy et al. (1998) find that those Tawahkan Indian who are involved in the market through wage labour have much less knowledge of plants, animals and their interactions when compared with those focused solely on traditional livelihoods. However it is worth noting that in some cases, market integration may also lead to TEK exchange between communities (e.g. Guest, 2002), or to increased local ecological knowledge of marketable goods (e.g. Ito, 2004).

Exposure to markets may also change an indigenous population's value systems. According to Shanley and Rosa (2004), where commercial logging occurs and the trade in non-timber products declines in forested areas of the lower Brazilian Amazon, young people and employees of logging companies tend to place higher value on the commercial timber species, while older and economically independent people may continue to more highly value non-timber species (e.g. medicinal species). 
These findings are supported by the survey responses. Fifty-nine percent of the responses identified that reliance on modern products and technologies has changed the traditional livelihood practices significantly. For example, a researcher from New Zealand stated, "[Maori people nowadays have] more reliance on processed foods and hunter-gathering in supermarkets. Fishing is more a recreation than a staple foodgathering need". A similar trend is also perceived by a researcher who works in Huamachuco and surrounding villages in La Libertad of Peru, "There is increasing access to 'Western' medicine for those with higher incomes. However, for those with lower incomes, mainly farmers in those villages furthest from the main town, medicinal plant knowledge is strong".

Reduced land-based or sea-based activities were identified by $22 \%$ of the responses. As many respondents stated, due to the availability of factory work and access to the market, many indigenous people are shifting away from their traditional livelihoods (e.g. agriculture, fisheries, pastoralism and agri-pastoralism), which carry more land or seabased traditional knowledge. A researcher from Campeche of Mexico observed that, "many men are no longer farming, and are supporting families by participating in consumer economy rather than farming economy". In Khunjerab National Park in Northern Pakistan, "traditionally local people spent the whole summer in the High Alpine Pasture as shepherds. Their interactions with the land and animals taught them about the land ecology and behaviour of the wild animals. However, due to shifting trades, the shepherds are decreasing and people are getting rid of livestock".

Another cause of reduced land-based activities addressed by two survey responses is the formal education system and the time demands it places on families. As a researcher from Panama pointed out, “... formal education means the mothers have to stay home to help children get ready and meet them after school. So the family as a whole does not spend as much time working and living on the land". 
The use of Westernised primary production systems was also identified as a main threat to traditional livelihood by survey responses (19\%) and in the literature. According to an organisation that works in the West Bengal region of India, "Commercially designed High-Yielding Varieties (HYVs) have been introduced and replaced traditional crop varieties". This indicates that the knowledge and skills in association with traditional crops will decline or be lost. Similarly, Soemarwoto (2007) finds that HYVs of rice are replacing the traditional varieties in Kasepuhan, west Java, Indonesia. All Kasepuhan groups have accepted the use of HYVs, and the traditional swidden cultivation practices have been abandoned. However, unlike the swidden practice which developed to combat irregular environmental conditions and climatic stress, the use of HYVs has confronted them with pest disease infestations and crop failure (Iskandar, 2007). This example raises an important concern about the threshold of a TEK system. Sometimes, the abandonment of traditional practice over a limited period of time may not lead to permanent loss of knowledge (Groffman et al., 2006). However, if the practice is abandoned long enough (perhaps just one generation) the knowledge can be lost forever.

\subsubsection{Direct threat three - Change of traditional religion and beliefs}

In the typology, change of traditional religion and beliefs refers to loss or change of the traditional religion and beliefs with respect to the importance of nature and the local environment. As the ultimate level of traditional ecological knowledge, traditional beliefs, religion and worldviews are significant in maintaining TEK. However, the conversion or abandonment of traditional religion has been recorded widely, especially in the areas that have a history of colonisation, such as Africa, South Africa and Oceania (e.g. Ellen, 2007; Wavey, 1993). For instance, Chief Wavey (1993:12) stresses that, as a part of the colonisation processes in Canada, "in concert with the churches, Aboriginal children were removed from our communities year after year for the entire school season". Those children were prevented from speaking their language, and from practicing traditional ceremonies showing respect for Mother Earth and ancestors. The loss of traditional religion and beliefs in nature has become inevitable (Wavey, 1993). 
Tang and Gavin (2010) address the enforced abandonment of traditional Tibetan Buddhism, resulting in younger Mongolian generations have much less understanding of the land and the interdependence of complex ecosystems than when they had access to TEK embedded in traditional animistic beliefs.

Echoing the above studies, a community elder in Qinghai-Tibetan Plataea of China demonstrated, “In my parents' generation and my generation, everyone followed Tibetan Buddhism, which believes in the equity of all life forms, and respect to the nature. From my son's generation, they believe more in communism instead of Buddhism. And to my grandchildren' generation, they believe no more in communism, but in Western stars". As a researcher who works with indigenous communities along Negro river and Purus River at Amazonas state in Brazil described, "[The reason for TEK loss in the location is the] influence of missionaries from protestant religions, mainly. This is leading to significant changes in the worldview, beliefs and consequently, in the way of living of these indigenous peoples".

\subsubsection{Direct threat four - Change of environment and natural resources}

Change of environment and natural resources refers to shift of physical environment due to relocation, and/or degradation or depletion of natural resources. The loss of traditional habitat and depletion of biodiversity due to human activities (mostly by nonindigenous groups) has been recognised as an important cause of TEK degradation in many studies (e.g. Harrison, 2007; Lykke et al, 2004; Rocha Silva and Andrade, 2006; Shanley and Rosa, 2004; Zent and Maffi, 2009). TEK is a particularly local-context-based knowledge system, in which local environment and natural resources provide the foundation for the observation and knowledge of such to be developed over generations (Inglis, 1993). Thus, any change of this foundation will affect the continuity and survival of the local knowledge system. For instance, Rocha Silva and Andrade (2006) examine the cultural significance indices of useful plants in several communities in north-eastern Brazil. By comparing the indices of introduced and native plants in relation to the degree of deforestation and proximity to urban areas, they conclude that the loss of 
knowledge of native plants is caused by deforestation of the Atlantic forest ecosystem. In another region of northern Brazil (Cabolco communities), Shanley and Rosa (2004) recorded older informants' perception of the scarcity or disappearance of formerly highly valued plant species and game animals. This region is also suffering from deforestation. They conclude that the decline in the use of traditional knowledge is caused by habitat conversion and local species extinction.

Some survey respondents noted a link between immigration into indigenous territories, population growth, and natural resource degradation. A community member from Mwanza region of Tanzania perceived, "Since the immigrants entered the area, over use of natural resources especially vegetation of economic value has begun. The traditional use of these plant species is not guaranteed". In Kahua, Makira Ulawa Province of Solomon Islands, a researcher noted the "effects of needing to cope with decreasing resource availability due to population growth, which means people need to rely more on monetary economy, which changes emphasis on human-environment interactions".

The change of environment and natural resources may also be caused by relocation, both voluntary and forced. For example, a community member in Alashan region, Inner Mongolia of China stated, "The government enforced the relocation policy in 1996. About 100 Mongolian herders in our community were removed from the Helan mountain area to the mountain foot plain area. We are not allowed to access our traditional pastures any more, and had to convert to agricultural activities as the government arranged". Similarly claimed by a researcher from Indiana and Oklahoma of USA, "There was a vast removal of the Myaamia to reservations, on lands that are ecologically different from their traditional environment". In these two cases, as a result, the knowledge and skills about traditional landscape and livelihood of the indigenous groups will have a limited chance to survive.

\subsubsection{Direct threat five - Loss of traditional rights}

Loss of traditional rights refers to complete or partial loss of traditional land, loss of 
resource or resource rights, and loss of traditional access to natural resources. Many indigenous peoples or their ancestors have experienced or are experiencing vast loss of their traditional rights. Such loss may include invasion of their homelands (e.g. colonisation), dispossession of lands and resources (e.g. forced nationalisation processes), and discrimination against their traditional life ways, culture and traditions (Colchester, 2000; Kastrup, 1997; Migot-Adholla et al., 1991; Pasqualucci, 2006; Sjaastad and Bromley, 1997; Trask, 2009). For most of the indigenous peoples, they either adapt to new life ways or face being marginalised (Kastrup, 1997).

The grave threat posed by loss of traditional rights was recognised by the Department of Economic and Social Affairs (DESA) of the United Nations in its report - State of World's Indigenous Peoples (2009:225), "While there is a great diversity of problems confronting indigenous peoples today, it is clear that one of the most significant threats faced by indigenous peoples arises from their displacement, eviction and separation from their lands, territories and resources. These issues are expanding and represent significant challenges to the security, health and survival of indigenous peoples and their cultures". The report lists five of the most common causes of indigenous peoples' dispossession and displacement: violence and militarism, becoming conservation refugees, migration and urbanisation, globalisation, and indigenous peoples living in voluntary isolation. I will provide detailed analysis of the three most significant causes below.

In nearly every region of the world, indigenous peoples are being displaced and severely impacted by violence and militarism, which directly threatens the traditional lifestyles and survival of indigenous communities (Trask, 2009). For instance, Sayad (2004) provides an example of the historical processes of French occupation of Algerian territory achieved through military violence, massacres, and destruction of the traditional society. Such violent occupation commonly took place during the colonial periods in many indigenous territories in Africa, Asia and the Americas. The occupation of indigenous territories by armed forces often results in the military restricting movement of members of the community engaged in hunting, fishing, or the gathering 
of plants for traditional medicines (Rights and Democracy, 2006, section 9). Militarism has also been used to gain control over natural resources, including land, minerals and oil, without restitution or compensation to its indigenous owners until the present time (WGIP, 2006).

Unfortunately, in many locations formal nature conservation has had negative impacts on indigenous people. In recent years, the expansion of protected areas has grown phenomenally. In 1962, there were 1,000 officially protected areas worldwide, but by 2003 , the number increased to 102,102, covering $12 \%$ of the earth's surface (Bowie, 2006; Huertas, 2004). Among these protected areas, about $85 \%$ are inhabited by indigenous people (Alcorn, 2000), and most remaining areas of the world's tropical forests with high biodiversity are also owned or claimed by indigenous peoples (Weber and Larson, 2000). The conservation of wildlife and preservation of national parks often involve evicting indigenous people, separating them from the natural resources they relied on previously. These natural conservation actions have generated a great deal of social conflict between indigenous communities and conservation authorities worldwide (Stavenhangen, 2007b).

For example, a community member from Aberdare National Park in Kenya noted that, "Since the National Park was established in the 1950s, our traditional hunting activities have been banned. We hardly have any other sources of living. The park is under the management of the Kenya Wildlife Service, in which no local community is involved". Similarly, a researcher working in the interior Koyukon Athabascan villages in Alaska of USA said that, "[there are many] oppressive non-Native policies, such as control of wildlife management, and the division of land into state and federal. These policies have largely led to the land and resources are more available to non-Native users rather than to the local tribes".

Migration and urbanisation are other major mechanisms through which indigenous peoples are separated from their traditional land and resources. In many cases, the migration and urbanisation processes are involuntary. Such processes may happen in 
two ways: 1) more commonly, indigenous people move to urban areas due to environmental degradation that has destroyed traditional livelihoods. This may be due to dispossession, displacement, military conflict or natural disasters (UNPFII, 2007). These indigenous migrants often find themselves encountering substantial difficulties, such as lack of employment, insufficient income, racism, and limited access to services (UNPFII, 2007); 2) non-indigenous immigrants move to indigenous lands and impose new land-uses based on Westernised development models. Indigenous people are often marginalised from this development, if not totally excluded (UNPFII, 2005). For example, an indigenous community member from Maryland in the USA described how "the access to our traditional resource is decreasing as people from outside of the area are moving in and buying properties. They are making the land no longer available to locals for harvesting".

Suppression of indigenous populations has taken place for several centuries, and only in the last few decades has the international community recognised indigenous peoples' rights (inter alia) to the use, ownership, management, and control of their traditional lands and territories (Kastrup, 1997; Lawrey, 1990; Pasqualucci, 2006). For example, the International Labour Organisation Convention No.170 is the first international treaty that specifically deals with indigenous rights (International Labour Organisation, 1989). It recognises indigenous peoples' rights of ownership, collective or individual, of the lands they traditionally occupy (Article 11). In many ways the Rio Earth Summit (1992) was a turning point for indigenous people. Not only the indigenous peoples were recognised as a "major group" of civil society but, for the first time, they were able to participate in and influence processes relating to the environment (DESA, 2009). Following the Earth Summit, both the Rio Declaration and the Convention on Biological Diversity (CBD, 1992) recognised the unique relationship between indigenous people and their traditional lands. They also established international legal standards for protecting indigenous peoples' rights to their traditional knowledge and practices in the areas of environmental management and conservation (DESA, 2009). However, as many scholars point out, translating such principles is not easy (e.g. Colchester, 2000). This is mainly because conventional governmental policies, laws and institutions entrenched 
under the old exclusionist model have not kept pace with improvements in international human rights law and conservation standards (Colchester and Erni, 1999; Gray et al., 1998).

\subsubsection{Direct threat six - Change of traditional institutions}

Change of traditional institutions refers to complete or partial loss, or change in traditional social organisation, customary norms and traditional rules of resource use and management. Even though the loss of traditional institutions has only been recognised as a threat to a limited degree in the literature ( $4 \%$ of the studies) and by survey respondents $(6 \%)$, the importance of maintaining traditional institutions is significant. Social institutions are a critical component of indigenous cultural identity. Although great heterogeneity exists among different indigenous groups, there are several common attributes of indigenous institutions shared by many indigenous societies. Indigenous institutions usually reflect holistic worldviews and collective perspectives, which include an interactive and respectful relationship between the community, nature and ancestors (Gomez, 2007). In indigenous societies, there commonly exists a wide range of social institutions for reciprocity and mutual aid (Schwab, 1995), sharing food and other resources, resolving conflicts and administering justice, and for managing commonly held resources (Martin, 1995). Indigenous rules and regulations are often based on extensive consultation and discussion among the group members, with all adults, and sometimes children also, having the opportunity to participate. Decision-making is often done on the basis of consensus (Stavenhagen, 2005). Traditionally, indigenous communities had people whom they respected and whose suggestions they usually chose to abide by. Customs and customary laws arising from such social organisation were generally accepted and followed, which limited the occurrence of serious social conflict (Assimeng, 1997; Kendie and Guri, 2006). All these unique attributes of indigenous institutions have been advantageous in achieving social stability and more sustainable management of common resources (Kendie and Guri, 2006; Ostrom, 1990, 2009). 
However, along with colonisation and the wide application of non-indigenous institutional structures, indigenous social institutions have largely been replaced and subordinated to modern state structures (Kipuri, 2009). Centralised systems of governance are now the dominant society's norm, and these have been replicated from the central to the local level, leaving little power or influence to traditional leaders. In many countries, indigenous customary laws have been forbidden or subordinated to the formal state legal system; social institutions related to family, education, health and economy have disappeared, changed in accordance with mainstream norms, or been weakened to the extent of losing their significance (Kipuri, 2009). This process of traditional institutional devolution has left many indigenous societies severely disrupted culturally and socially. If the process has been accompanied by the loss of land, territories and natural resources, the whole indigenous culture will inevitably be damaged (Stavenhagen, 2005). The loss of traditional institutions, as addressed by several survey respondents, is largely driven by governmental interference. For example, a community member from the lower North Island of New Zealand claimed, "Top-down resource governance replaced our traditional community-based resource use and management system. Thus, our traditional resource management practices can hardly be carried on".

A similar statement was also made by a researcher (surv. resp., 201028) working in the Borana region of South Ethiopia, who described how "the indigenous institutions that enabled indigenous pastoralists to operate in (the) resource-scarce region of Borana zone for centuries, have been eroded by modern innovations and governmental policies, including privatisation, settlement, cultivation (and) land annexation."

The weakening or loss of their traditional institutions has challenged indigenous peoples' distinct cultural identities. As a key factor of effective self-governance, the maintenance and protection of indigenous institutions therefore forms a crucial part of overall indigenous TEK conservation and indigenous rights protection.

\footnotetext{
${ }^{28}$ Surv. resp. is short for survey response.
} 
Even though they are not addressed evenly in the literature and by survey respondents, the above six direct threats are all inter-dependent and inter-connected with each other. They are therefore all highly important to TEK conservation efforts. More importantly, these direct threats should be dealt with in association with their underlying causes. By far the two most common direct threats noted by survey informants were loss of pathways of TEK transmission (37\%) and the change of traditional livelihood practices (34\%). The other four threats were mentioned with similar frequency, as identified in Figure 3.7 below.

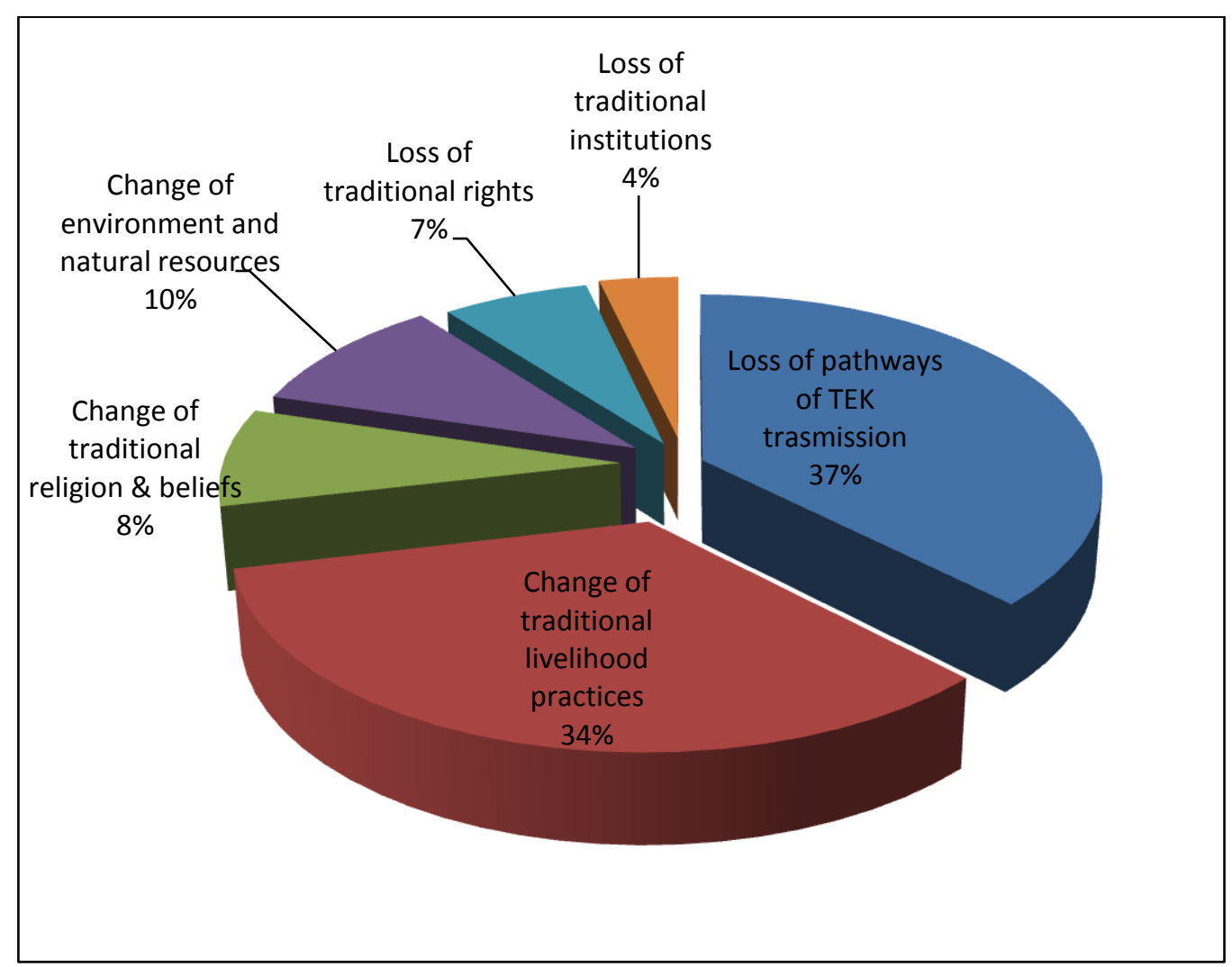

Figure 3.7 Ratio of direct threats to TEK - Results from the survey (n=137)

By applying the same analysis to the literature I reviewed, a similar pattern was found (see Figure 3.8 below). The top two threats identified in the literature were also loss of pathways of TEK transmission (44\%) and the change of traditional livelihood practices (21\%). The other four threats were also identified with similar frequencies, with only the order between change of traditional religion and belief and change of 
environment and natural resources reversed from the results of the survey. The results of the survey and literature review support each other by presenting a similar pattern of direct threats.

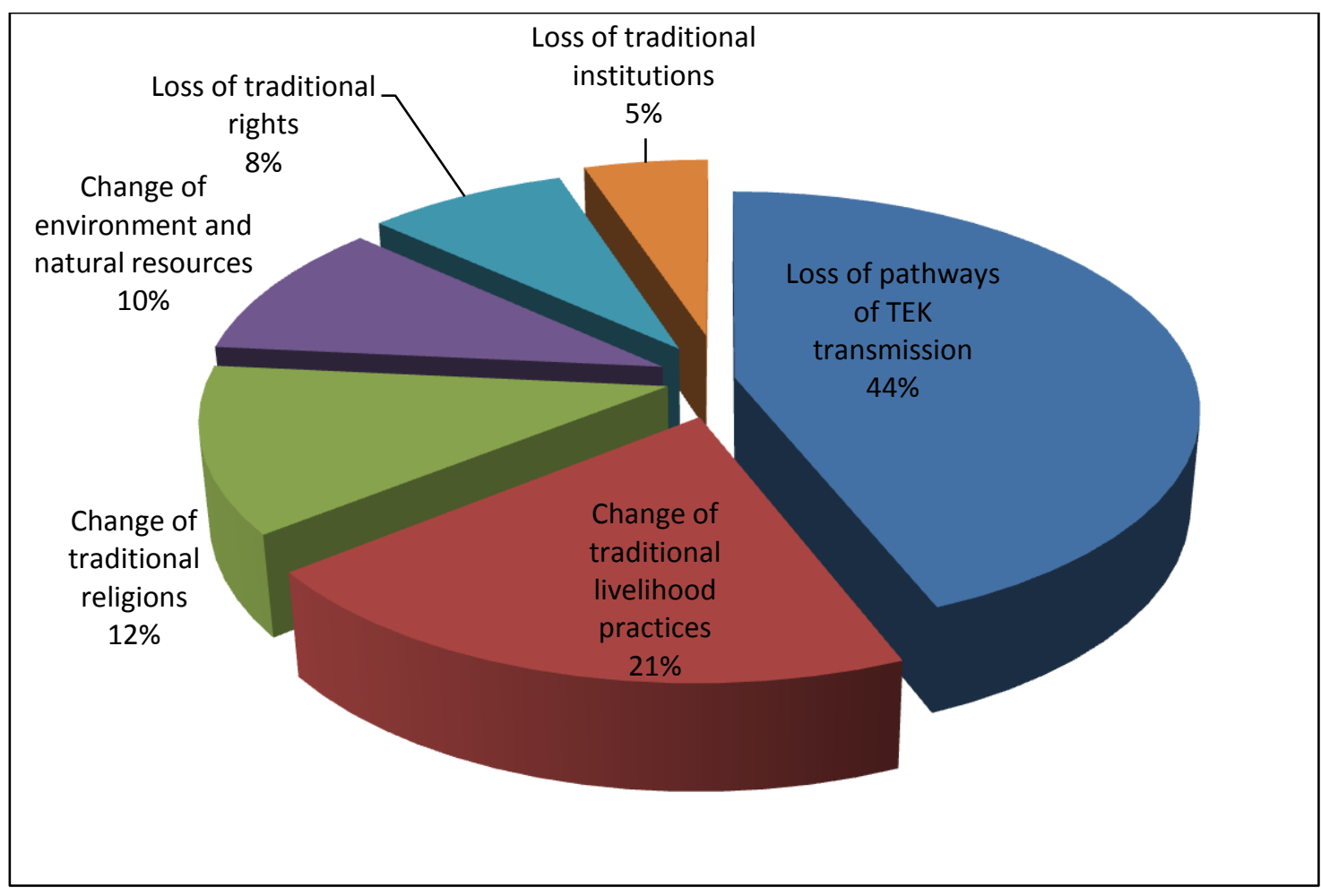

Figure 3.8 Ratio of direct threats to TEK - Results from literature review ( $n=111)$

\subsubsection{The underlying threats to TEK}

Ten distinct categories of underlying threats to TEK have been developed in this research. In each category, the typology lists all direct threats that may be caused by the underlying threat. The typology also provides examples of how underlying threats may cause direct threats. 
Table 3.2 The Typology of Underlying Threats to Traditional Ecological Knowledge

\begin{tabular}{|c|c|c|}
\hline Underlying Threats & $\begin{array}{l}\text { Direct Threats influenced } \\
\text { by the underlying threat }\end{array}$ & Examples \\
\hline \multirow[t]{4}{*}{$\begin{array}{l}\text { 1. Government policy and } \\
\text { legislation (policy and } \\
\text { legislation that devalue and/or } \\
\text { suppress indigenous groups } \\
\text { and their cultures ) }\end{array}$} & $\begin{array}{l}\text { Loss of pathways of TEK } \\
\text { transmission }\end{array}$ & $\begin{array}{l}\text { State government banning the use of } \\
\text { native language in public schools } \\
\text { causing the loss of traditional } \\
\text { language and TEK carried by that } \\
\text { language }\end{array}$ \\
\hline & $\begin{array}{l}\text { Change of traditional livelihood } \\
\text { practices }\end{array}$ & $\begin{array}{l}\text { A forced settlement policy changes } \\
\text { nomadic herders' traditional } \\
\text { livelihood practices }\end{array}$ \\
\hline & Loss of traditional rights & $\begin{array}{l}\text { Government claims state ownership of } \\
\text { indigenous lands }\end{array}$ \\
\hline & Loss of traditional institutions & $\begin{array}{l}\text { Government appointed leadership } \\
\text { replaced traditional social hierarchy } \\
\text { and leadership }\end{array}$ \\
\hline \multirow[t]{2}{*}{$\begin{array}{l}\text { 2. Contact with other cultural } \\
\text { groups (can be caused } \\
\text { deliberately by non-indigenous } \\
\text { groups or voluntarily by } \\
\text { indigenous groups) }\end{array}$} & $\begin{array}{l}\text { Loss of pathways of TEK } \\
\text { transmission }\end{array}$ & $\begin{array}{l}\text { Western TV programmes decrease } \\
\text { young indigenous people' incentives } \\
\text { and interests in traditional knowledge } \\
\text { and culture }\end{array}$ \\
\hline & $\begin{array}{l}\text { Loss of traditional religion and } \\
\text { beliefs }\end{array}$ & $\begin{array}{l}\text { Christian missionaries impose or } \\
\text { promote a change from traditional } \\
\text { beliefs to Christianity }\end{array}$ \\
\hline \multirow{2}{*}{$\begin{array}{l}\text { 3. Influence of outside } \\
\text { market (including the } \\
\text { availability of modern goods, } \\
\text { access to market and the } \\
\text { possibility of engaging with } \\
\text { trading activities) }\end{array}$} & $\begin{array}{l}\text { Loss of pathways of TEK } \\
\text { transmission }\end{array}$ & $\begin{array}{l}\text { The availability of processed foods } \\
\text { decreases incentives of learning and of } \\
\text { using traditional knowledge to make } \\
\text { own foods }\end{array}$ \\
\hline & $\begin{array}{l}\text { Change of traditional livelihood } \\
\text { practices }\end{array}$ & $\begin{array}{l}\text { Market demand facilitates the shift } \\
\text { from planting traditional crops (e.g. } \\
\text { taro) to producing cash crops (e.g. } \\
\text { potato) }\end{array}$ \\
\hline
\end{tabular}




\begin{tabular}{|c|c|c|}
\hline \multirow{5}{*}{$\begin{array}{l}\text { 4. Colonisation (the } \\
\text { establishment, maintenance, } \\
\text { acquisition and expansion of } \\
\text { colonies in one territory by } \\
\text { people from another territory) }\end{array}$} & $\begin{array}{l}\text { Loss of pathways of TEK } \\
\text { transmission }\end{array}$ & $\begin{array}{l}\text { Enforced Western education system } \\
\text { causes reduced TEK transmission }\end{array}$ \\
\hline & $\begin{array}{l}\text { Change of traditional livelihood } \\
\text { practices }\end{array}$ & Enforced industrialisation processes \\
\hline & $\begin{array}{l}\text { Loss of traditional religion and } \\
\text { beliefs }\end{array}$ & $\begin{array}{l}\text { Enforced or voluntarily change from } \\
\text { traditional belief to coloniser's } \\
\text { religion, such as Christianity }\end{array}$ \\
\hline & Loss of traditional rights & Loss of traditional land ownership \\
\hline & Loss of traditional institutions & $\begin{array}{l}\text { Natural resource governance is } \\
\text { controlled by the colonisers }\end{array}$ \\
\hline \multirow[t]{3}{*}{$\begin{array}{l}\text { 5. Relocation } \\
\text { (enforced or voluntarily) }\end{array}$} & $\begin{array}{l}\text { Change of traditional livelihood } \\
\text { practices }\end{array}$ & $\begin{array}{l}\text { Agro-forestry groups are relocated } \\
\text { from mountain areas to agricultural } \\
\text { areas, and have to practice } \\
\text { agricultural activities }\end{array}$ \\
\hline & Loss of traditional rights & $\begin{array}{l}\text { Indigenous people are removed from } \\
\text { their land for mining activities }\end{array}$ \\
\hline & $\begin{array}{l}\text { Change of environment and } \\
\text { natural resources }\end{array}$ & $\begin{array}{l}\text { Pastoral groups move to agricultural } \\
\text { areas as ecological refugees, and have } \\
\text { to learn about a new environment and } \\
\text { its resource use }\end{array}$ \\
\hline $\begin{array}{l}\text { 6. Marginalisation by } \\
\text { dominant societies (including } \\
\text { social, economic and political } \\
\text { marginalisation, which often } \\
\text { leads to reduced self-esteem of } \\
\text { indigenous population) }\end{array}$ & $\begin{array}{l}\text { Loss of pathways of TEK } \\
\text { transmission }\end{array}$ & $\begin{array}{l}\text { Under social racism, young indigenous } \\
\text { people feel ashamed about their } \\
\text { indigenous identity, and therefore } \\
\text { make more effort to learn mainstream } \\
\text { culture and skills instead of TEK }\end{array}$ \\
\hline $\begin{array}{l}\text { 7. War and military } \\
\text { occupation }\end{array}$ & Loss of traditional rights & $\begin{array}{l}\text { Military occupation of indigenous } \\
\text { lands }\end{array}$ \\
\hline $\begin{array}{l}\text { 8. Indigenous population } \\
\text { decline (including natural } \\
\text { demographic decline or human } \\
\text { made incidents, such as } \\
\text { genocide) }\end{array}$ & $\begin{array}{l}\text { Loss of pathways of TEK } \\
\text { transmission }\end{array}$ & $\begin{array}{l}\text { Elders pass away without transmitting } \\
\text { their knowledge to younger } \\
\text { generations }\end{array}$ \\
\hline
\end{tabular}




\begin{tabular}{|c|c|c|}
\hline \multirow[t]{4}{*}{$\begin{array}{l}\text { 9. Migration (including } \\
\text { indigenous emigration and non- } \\
\text { indigenous immigration) }\end{array}$} & $\begin{array}{l}\text { Loss of pathways of TEK } \\
\text { transmission }\end{array}$ & $\begin{array}{l}\text { Indigenous youngsters who move to } \\
\text { urban areas for jobs have decreased } \\
\text { incentives and interest in learning TEK }\end{array}$ \\
\hline & $\begin{array}{l}\text { Change in traditional livelihood } \\
\text { practices }\end{array}$ & $\begin{array}{l}\text { Indigenous people who move out from } \\
\text { their traditional lands have to adapt } \\
\text { their living to the new environment }\end{array}$ \\
\hline & Change in traditional religions & $\begin{array}{l}\text { Immigrants bring new religion into } \\
\text { indigenous populations }\end{array}$ \\
\hline & Loss of traditional rights & $\begin{array}{l}\text { An increased number of outsider } \\
\text { immigrants occupy the traditional } \\
\text { land-base of indigenous populations }\end{array}$ \\
\hline $\begin{array}{l}\text { 10. Economic development } \\
\text { pressure (sustained and } \\
\text { concerted actions that promote } \\
\text { the standard of living and } \\
\text { economic health of a specific } \\
\text { area, usually driven by the } \\
\text { dominant market economy) }\end{array}$ & $\begin{array}{l}\text { Change of traditional livelihood } \\
\text { practices }\end{array}$ & $\begin{array}{l}\text { Urbanisation changes traditional } \\
\text { farmland into city, and converts } \\
\text { traditional farmers into urban } \\
\text { labourers }\end{array}$ \\
\hline
\end{tabular}

According to the survey respondents, the most threatening factors are contact with other cultural groups, migration, influence of outside market, economic development pressure, and government policy and legislation (identification frequency > 10\%); the other five categories count fewer than $10 \%$ respectively (see Figure 3.9). 


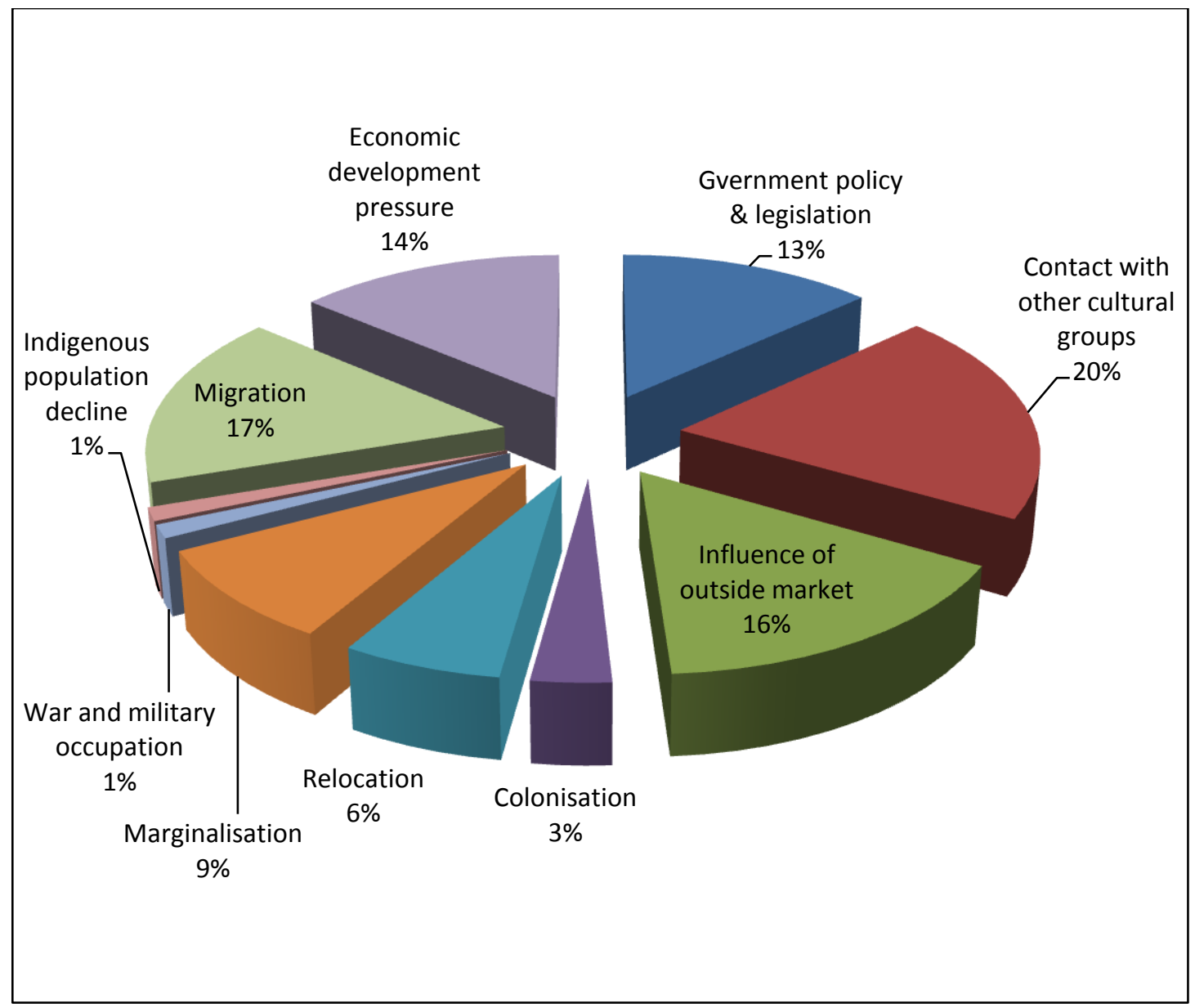

Figure 3.9 Ratio of various categories of underlying threats to TEK - Results from the survey $(n=137)$

The top four underlying threats identified in the literature were influence of outside market, migration, government policy and legislation, and contact with other cultural groups (identification frequency $\geq 10 \%$ ). Five other categories showed the same frequency (7\%), but none of the articles or studies I reviewed identified indigenous population decline as a threat (see Figure 3.10 below). The survey and literature review identify the same top three threats - contact with other cultural groups, influence of outside market, and migration, but in a slightly different order. 


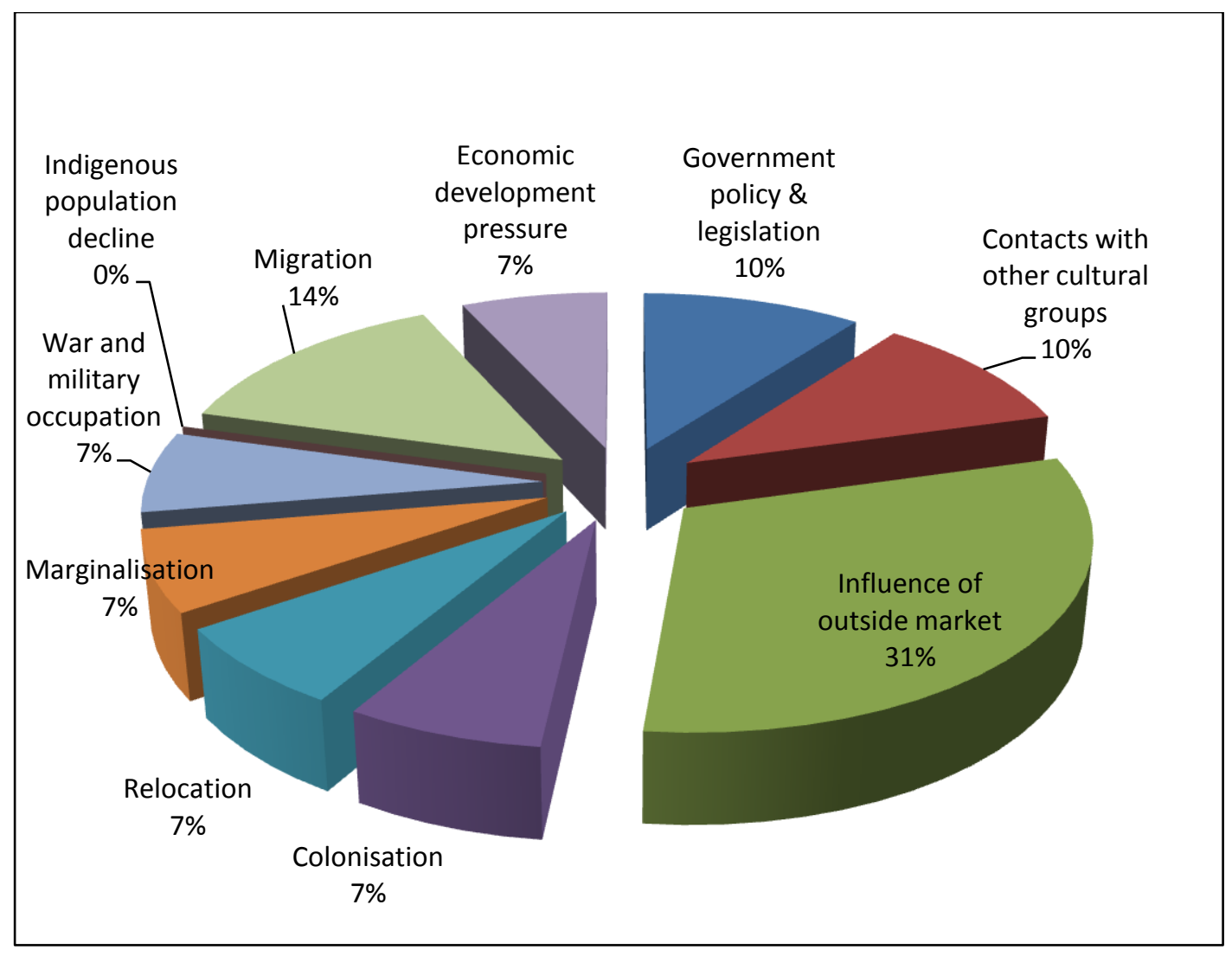

Figure 3.10 Ratio of various categories of underlying threats to TEK - Results from literature review $(n=152)$

After identifying all direct and underlying threats, it is very important to understand the inter-relationships between them. By identifying the causal relationships between direct threats and their underlying causes, it will be easier to prioritise conservation action targets; thereby achieving more effective of conservation efforts. I will present this complex inter-relationships web with detailed examples in the next section.

\subsection{Other results}

The survey also investigated which indigenous groups and what aspects of TEK have been studied. In total, about 164 distinct indigenous groups from 48 countries and regions were involved in the survey. A wide range of topics and disciplines were noted by the survey respondents, ranging from traditional knowledge of resources and land (e.g. folk medicine, bush-meat, local plant and animal inventory, sacred grove), 
traditional management practices (e.g. water resource management, forest management, adaptation to climate change, traditional use of fire, sustainable forestry and fishery), traditional institutions (e.g. traditional fishing quota, traditional wildlife policy), cultural heritage (e.g. language immersion, traditional religion-based daily practices), to the protection of TEK (e.g. integrating TEK with Western science, intellectual property rights of indigenous people, cultural heritage rights). These studies cover all the different dimensions and aspects of TEK (please see additional details in Appendices 6 \& 7).

\section{DISCUSSION \& CONCLUSION}

The TEK threat typology developed in this study is an initial effort to identify and present TEK threats in a systematic way. It then provides a foundation for further analysis of TEK threats, which informs TEK conservation. The users of the typology can scan through the TEK typology to identify and describe the threats that occur at their sites. Once the threats have been identified, conservation actors can, in association with the use of the conservation action typology, identify and prioritise actions to deal with the threats.

In this section, I will first demonstrate the causal relationships between direct and underlying threats to TEK; then use examples with flow chart illustrations to demonstrate the possible threat pathways under each direct threat. Identifying the threat pathways makes it easier to prioritise conservation actions and thereby achieve more effective conservation outcomes. Discussion of the driving forces of threats dynamics and potential user groups of the typology will be provided at the end of this section. 


\subsection{The complex system of inter-related TEK threats}

One of the most critical outcomes of creating the TEK classification system is to note that TEK degradation is a complex, multi-scalar problem, in which various threats act simultaneously. The causal relationships between underlying and direct threats are also complex. One direct threat may be caused by one or multiple underlying threats. Every underlying threat may also be caused by other underlying threats (see Figure 3.11 to 3.17). Without an in-depth understanding of both direct and underlying threats, as well as the inter-relationship between them, conservation actions may be misguided or limited in their impact. 


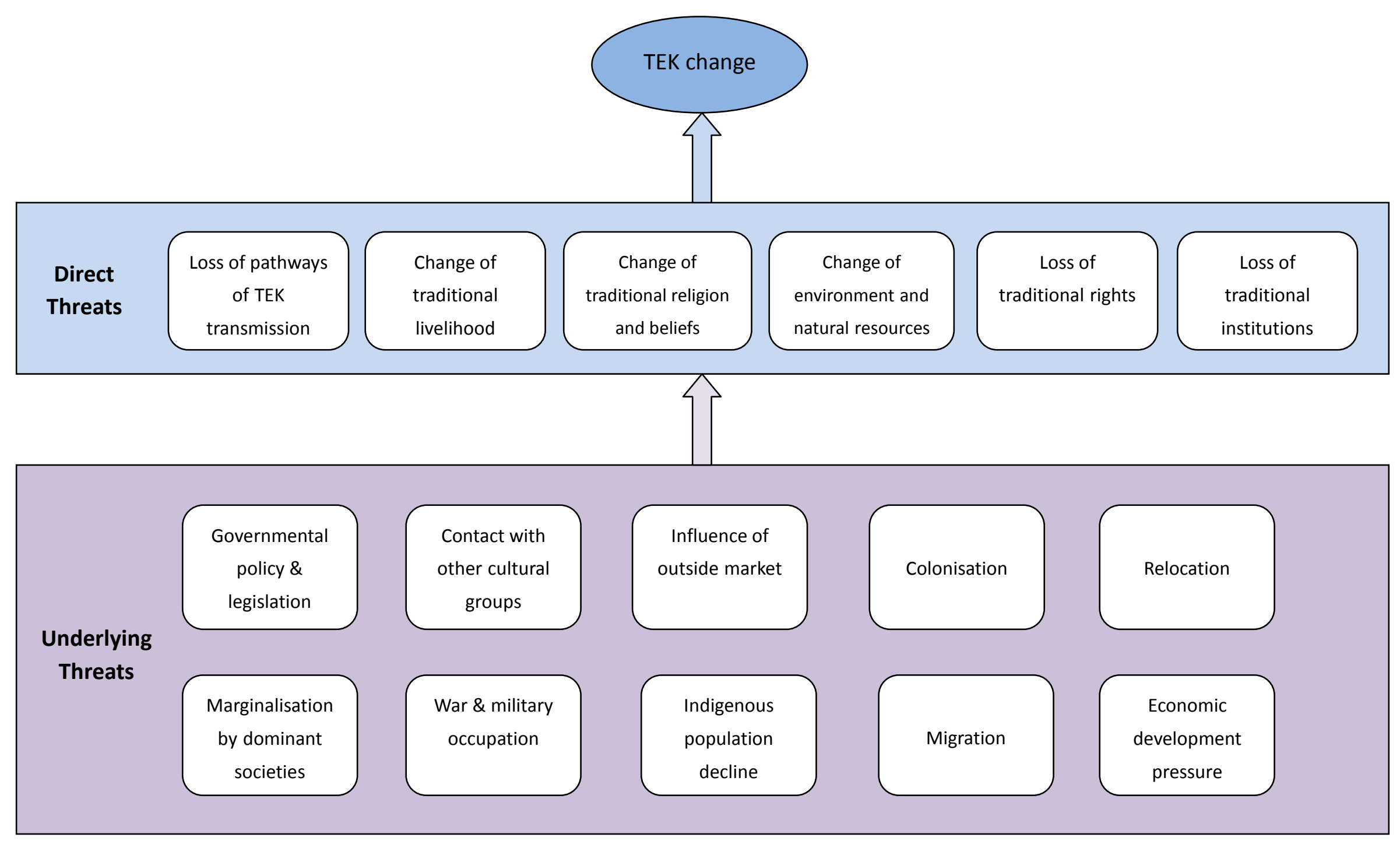

Figure 3.11 A model of the causality driving TEK change. 
The above figure provides an overview of the inter-relationships between TEK threats. Below, I use six examples with flow charts to illustrate the possible threat pathways under each direct threat (see Figures 3.12 to 3.17). Please note that the examples and the flow charts tend to extract individual direct threats for analytical purposes. In realworld scenarios, multiple direct threats often take place simultaneously, and the threat charts should be more like the complicated web presented above. Also, the examples merely highlight one or several pathways of the threats, rather than a comprehensive causal web. This may be partially because the studies from the literature and perceptions from the survey respondents usually focus on certain aspects based on their disciplinary background and context. Based on the information available it is usually difficult to obtain an in-depth overview of the issue and to carry out a more comprehensive analysis. By demonstrating the inter-connected nature of TEK threats and the importance of both direct threats and underlying causes, I hope this classification effort can encourage more research that attempts to uncover the entire causal web driving TEK change in focal locations. In this way the classification systems can help empower more effective TEK conservation action, as discussed in more detail in the next chapter.

\subsubsection{Threat pathways of loss of pathways of TEK transmission}

The loss of pathways of TEK transmission can be caused by any single threat or any combination of several underlying threats (see Figure 3.12). There is a causality web in all cases, and the exact web will vary from one context to another. Below, I use one example from the survey to illustrate how this causality web flows. In response to my survey, a community elder-leader from Tete Juan, British Columbia, Canada provided a detailed explanation of how the degradation of TEK transmission has happened in his community over generations, "Prior to my mother's generation, all community members 
spoke fluent native language, and understood our cultures. But due to residential school, and cultural genocide we had less fully culturally people in our community. In my mother's generation, $50 \%$ of our people spoke the language, but only $75 \%$ of these people were fluent. People started to move away from the traditional land and cultural based lifestyle. In my generation, $40 \%$ of the people speak the language with $50 \%$ of them are fluent. Most of us moved away from traditional use of the land and culture. There are only 15 people at my age left in the community now. My children's generation has only $30 \%$ who speak the language, and only $25 \%$ are fluent. None [of them] are living on the traditional land. They are living off the land in a modern way, and only come back to attend social affairs a few times of the year. Almost no one speaks the language outside of cultural events. My grandchildren's generation has only $10 \%$ who speak our language. Many [of them are still] too young yet to understand [the traditional cultures] but we are teaching this generation".

In this case the elder highlighted loss of traditional language as one of the key driving factors in the loss of TEK transmission. The decline of the number of native speakers and the dilution of fluency in the native language indicate the loss of traditional knowledge and culture that is carried by the language. As the elder claimed, the language loss is a result of residential school (influence of formal education) and cultural genocide which is caused by colonisation and/or contact with other cultural groups. Another main cause of the degraded TEK transmission addressed by the elder is that younger generations are moving away from traditional land and are living a modern lifestyle (younger generation's absence from the community; migration). Though this observation conducted by the elder might not cover all aspects of the issue, it undoubtly provided a grounded perception and understanding of the local context. Based on his analysis, we can develop a causal flow below: 


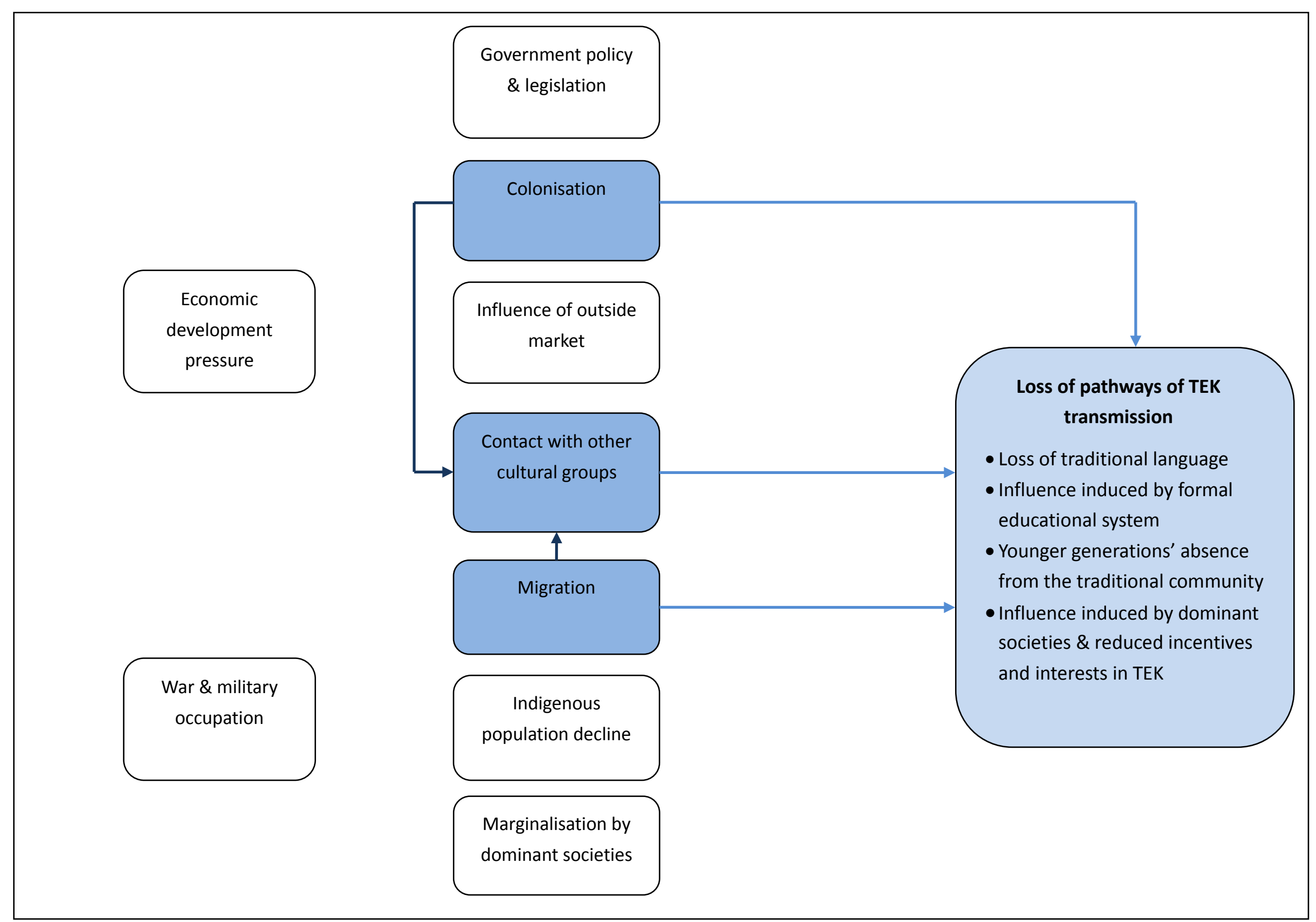

Figure 3.12 An example of the threat pathways of Loss of pathways of TEK transmission 
In this case, the loss of pathways of TEK transmission was caused by a combination of three underlying threats: contact with other cultural groups, colonisation and migration. Among these three, the contact with other cultural groups can be caused by migration processes and/or colonisation.

Following a similar format to the above discussion, I will provide an example and a flow chart with analysis for each direct threat respectively.

\subsubsection{Threat pathway of change of traditional livelihood practices}

The change of traditional livelihood practices may be caused by government policy and legislation, influence of outside market, relocation or migration, or any combination of these four underlying threats. Meanwhile, relocation and migration can be caused by colonisation processes and/or government policy and legislation; and government policy and legislation may be driven by economic development pressure.

Based on a case study of the Bali people of Venezuela, Lizarradle found that (1997), a significant cause of the loss of local ethnobotanical knowledge is the shift in local subsistence patterns. This is represented by change of traditional livelihood practices in the threat typology. As he points out, the open access to markets makes Western goods available to the community. The increasing consumption of Western goods then generates the need for cash. As a result, a cash-crop economy has been introduced, which is gradually replacing the traditional forest-resource-based economy. This shift, as Lizarradle stresses (2001), induces many young people to learn the country's dominant language, Spanish and new ways of life. According to his estimate (2001: 273), "the real loss of ethnobotanical knowledge from one generation to the next generation may be (in) the order of 40-60 per cent". 
The threat pathway based on this example will be rather simple, a direct line between influence of outside market and change of traditional livelihood practices. The outside market influence is likely to be driven by overall economic development pressure in the country and may also be internationally-driven (see Figure 3.13 below). This example highlights the causal relationship between market influence and traditional practices; but it does not mean that other threats do not influence the TEK system and lead to the change of livelihood practices. The research also points out that people with greater bilingual ability (Bali and Spanish) and more formal education have relatively less ethnobotanical knowledge, which can be categorised under the last direct threat, loss of pathways of TEK transmission. 


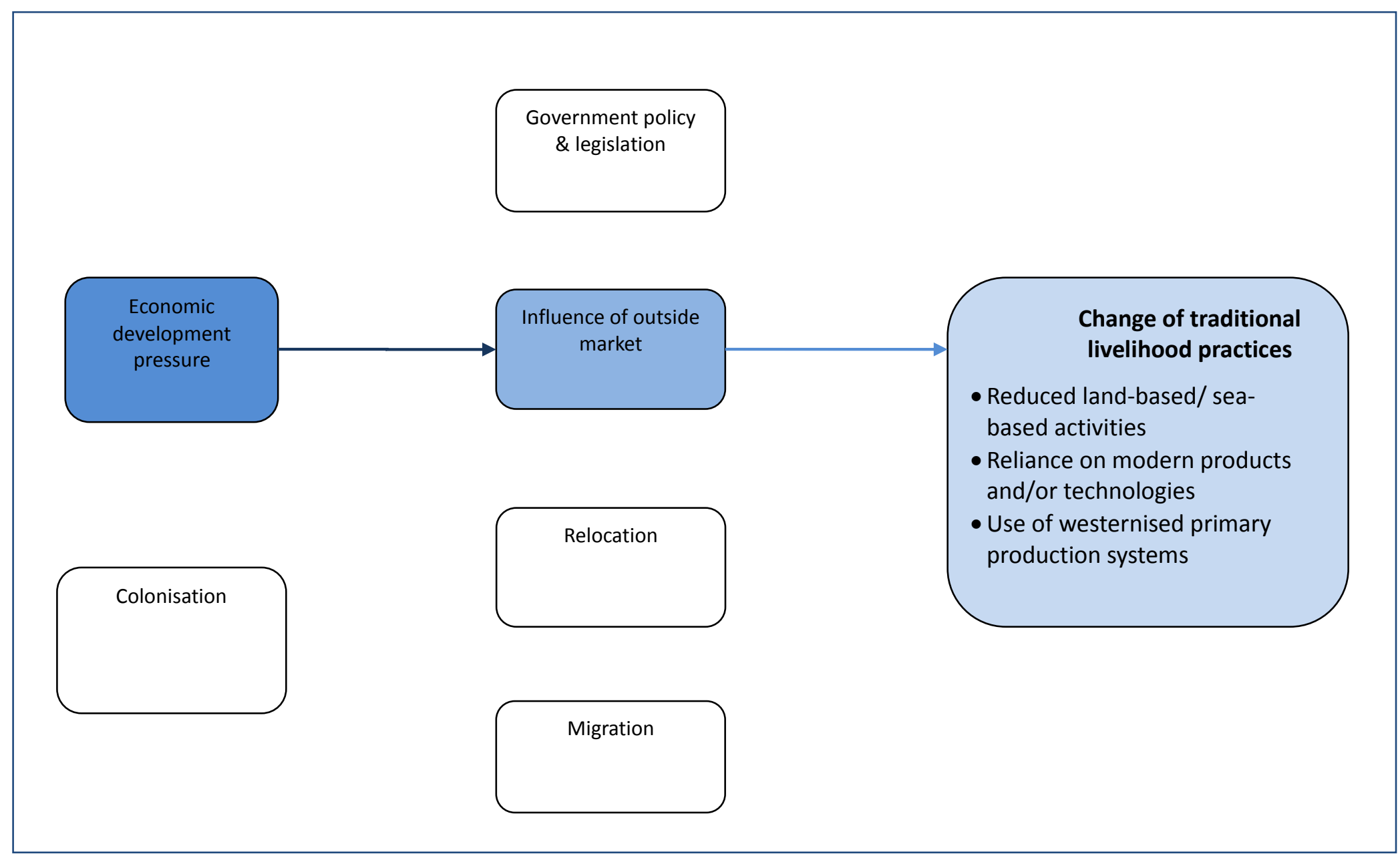

Figure 3.13 An example of the threat pathways of Change of traditional livelihood practices 


\subsubsection{Threat pathways of change of traditional religion and beliefs}

The change of traditional religion and beliefs can be either forced, voluntary, or both. Forced change commonly takes place through the colonisation processes; whereas voluntary change usually occurs as a gradual process of contact with other cultural groups and/or through migration processes. Looking deeper into their root causes, cultural contact and migration can also be driven by colonisation processes.

As Case et al.(2005) and Minol (2000) note, since the Manus (Papua New Guinea) have been exposed to and in some periods subjected to Western culture for nearly a century (including German colonisation, anthropological study and armed infiltration during World War II), medicinal plant knowledge has been disappearing rapidly over an extended period of acculturation. As Minol (2000) points out, Christian missionaries have continuously encouraged (sometimes coerced) the indigenous Manus to convert to Christianity. Meanwhile, the missionaries have also discredited the authority and prestige of native healers. As a result, the use and transmission of medicinal plant knowledge carried by healing practitioners have largely been undermined (Case et al., 2005). Also during the colonisation process, a significant number of immigrants entered the region. The indigenous groups were confronted with different religious and cultural practices that immigrants brought (contact with other cultural groups). All these activities have contributed to the abandonment of indigenous religions and the loss of indigenous knowledge (Case et al., 2005; Minol, 2000). 


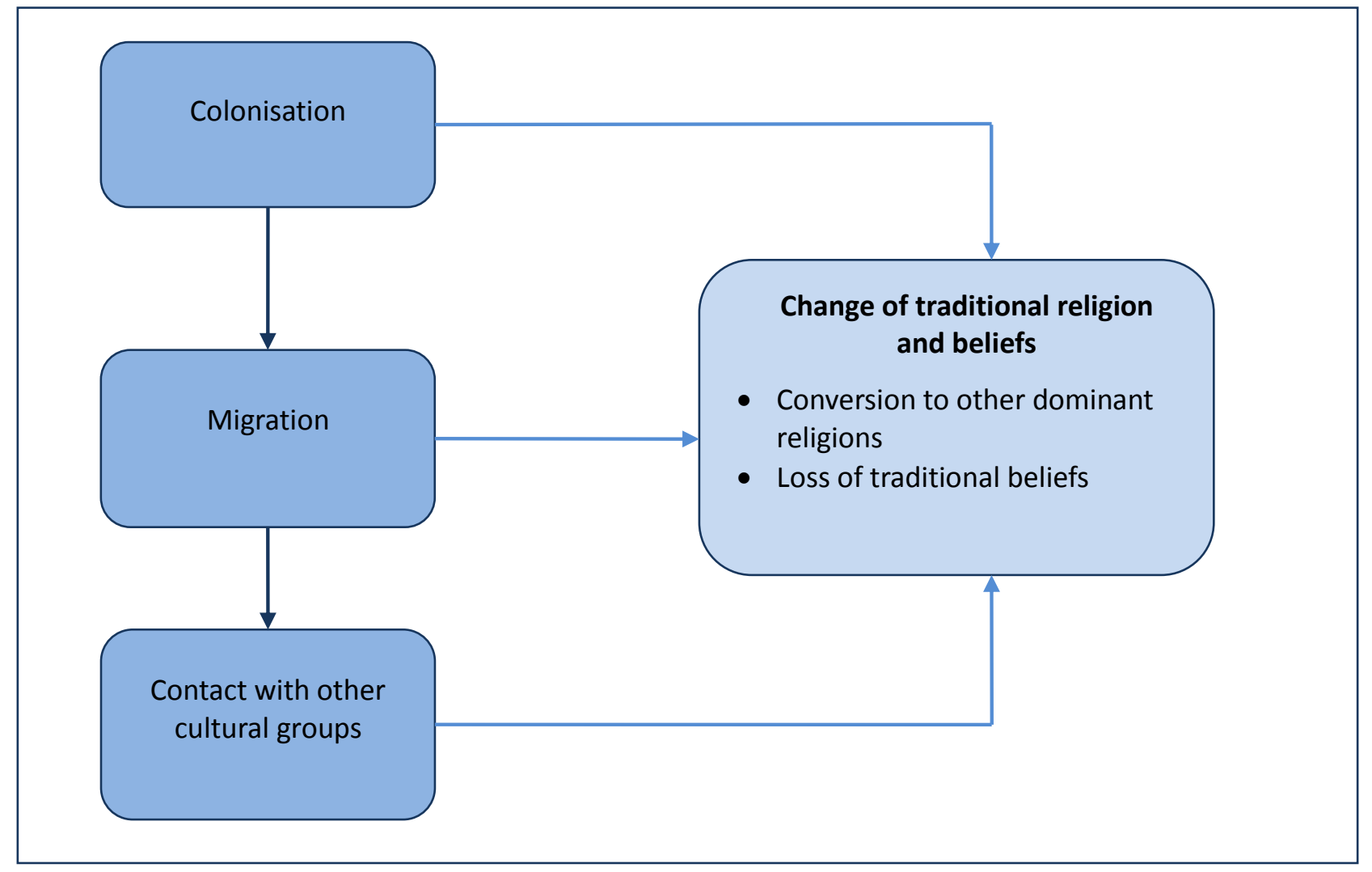

Figure 3.14 An example of the threat pathways of Change of traditional religion and beliefs 


\subsubsection{Threat pathways of change of environment and natural resources}

Change of environment and natural resources may be caused by government policy and legislation, colonisation, relocation, or migration; or any combination of these underlying factors. Meanwhile, government policy and legislation, relocation and migration might be caused by economic development pressure; and relocation can also be caused by government policy and legislation.

In this section, I use a case study in Qinghai-Tibet plateau as an example for analysis. Focusing on the land-use, livelihood and health transitions among Tibetan nomads in Qinghai-Tibet Plateau, Xu et al. (2008a:104) find that among the most powerful factors affecting local livelihood and traditional practices are "contemporary policies that aim to settle, standardise, and upscale rural population to meet the increasing demands of the local population and expansion of the market economy". One of the key policies over past decades has been to sedentarise and relocate Tibetan nomads and to restrict the movement of livestock and people. Among other things, relocating Tibetan nomads to sedentary villages is of symbolic value, as the state authorities see it as a measure of their integration into majority Chinese society and into modern development. This process has been widely applied in China since the Economic Reforms in the 1980s, when the national policies have been largely driven by economic growth. As a result, many nomads have moved from mountain areas to villages in lower lands. The environment and available resources are much different from their traditional areas. The change of environment has led to nomads' conversion to a sedentary, farming lifestyle. During this relocation and setenterisation process, traditional nomadic practices, and the knowledge and institutions associated with it, have been lost.

In this case, the change of physical location and natural environment of indigenous Tibetan nomads is directly caused by relocation, which is caused by government policy and legislation (see Figure 3.15). Meanwhile, such policy and legislation are largely driven by regional and national economic development pressure. 


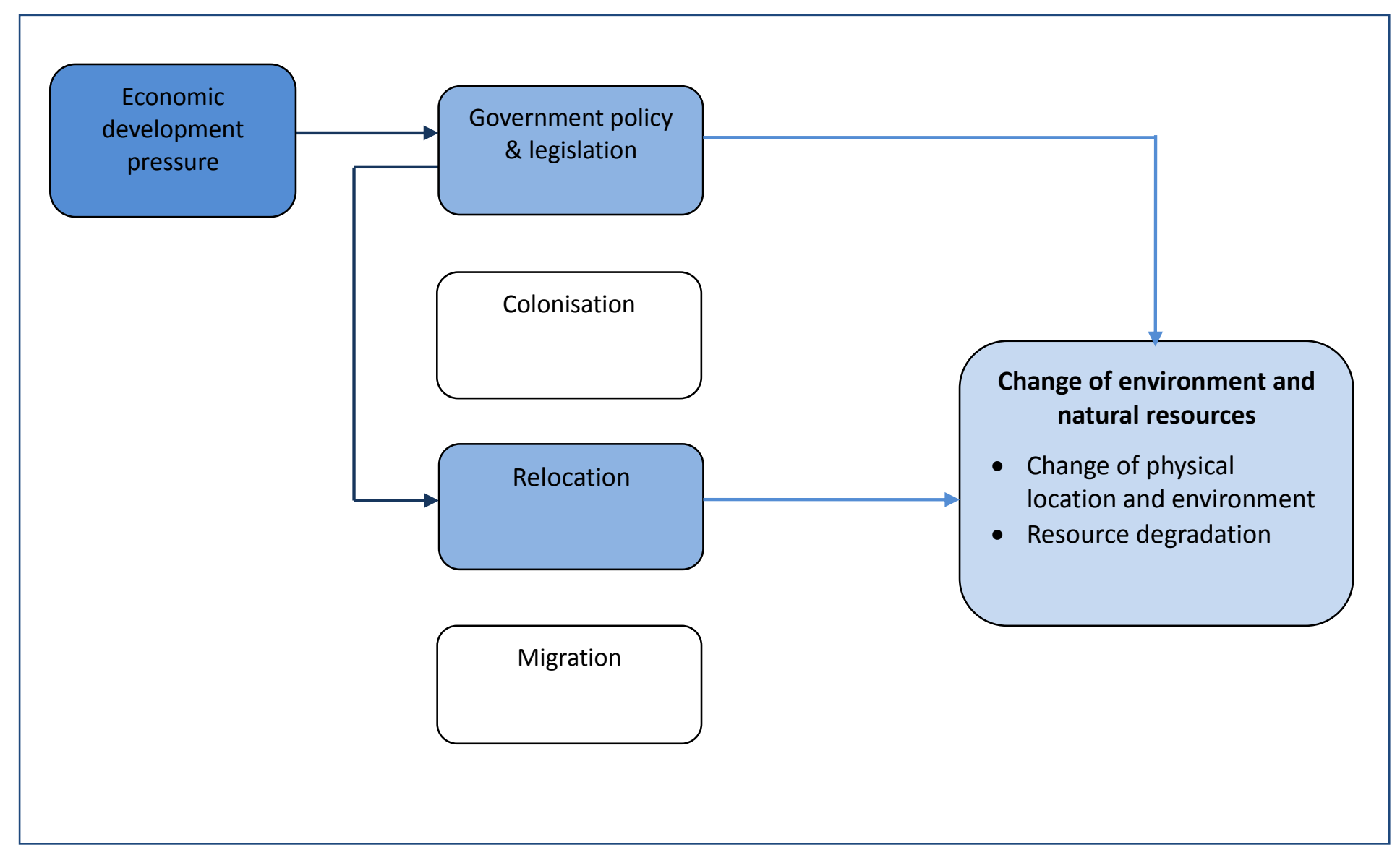

Figure 3.15 An example of the threat pathways of Change of environment and natural resources 


\subsubsection{Threat pathways of loss of traditional rights}

The loss of traditional rights can be caused by government policy and legislation, colonisation, relocation, or war and military occupation; or any combination of these threats. In the meantime, economic development pressure may function as a driving force of government policy and legislation; and relocation can be driven by government policy and legislation.

In this section, an example from Kenya is used for analysis. Kenya is widely admired for its numerous national parks and game reserves, which have become a major tourist attraction and therefore are important for the national economy (Trask, 2009). According to an estimate (Stavenhagen, 2007b), protected areas cover over 3.5 million hectares (about 6\% of Kenya's total land area); the direct and indirect revenues from wildlife conservation activities amount to $10 \%$ of Kenya's GDP (Trask, 2009). The conservation of wildlife and the preservation of protected areas are considered high priorities for the country, and have involved separating indigenous peoples from wildlife and wildlife habitats. Many families were evicted from newly created protected areas, most of which were originally inhabited by pastoralists and hunter-gatherers (Mukundi, 2009).

Based on the mistaken assumption, held since colonial times, that subsistence hunting by indigenous communities is devastating wildlife; the Wildlife Conservation Act prohibits game-hunting inside and outside the protected areas. Those indigenous people who access the resources and continue hunting are often arrested and accused of being "poachers" (Trask, 2009). Despite centuries of coexistence with wildlife, nomadic pastoralists are no longer allowed to herd their livestock in the reserves, even in game reserves which are managed by the local authorities on behalf of local communities (Stavenhagen, 2007b). Local indigenous communities do not participate in the management of the parks and reserves, and have no share in the conservation revenue. The revenue usually accrues to the Kenya Wildlife Service in the case of national parks, 
or to the local districts in the case of national reserves (Stavenhagen, 2007a).

In this case, the indigenous rights to use and to access natural resources for subsistence use are hampered or terminated due to the implementation of government conservation policy and legislation. Through eviction (relocation), restriction of access to resources and restriction of movement, indigenous peoples in Kenya have been dispossessed of their lands, territories and resources. 


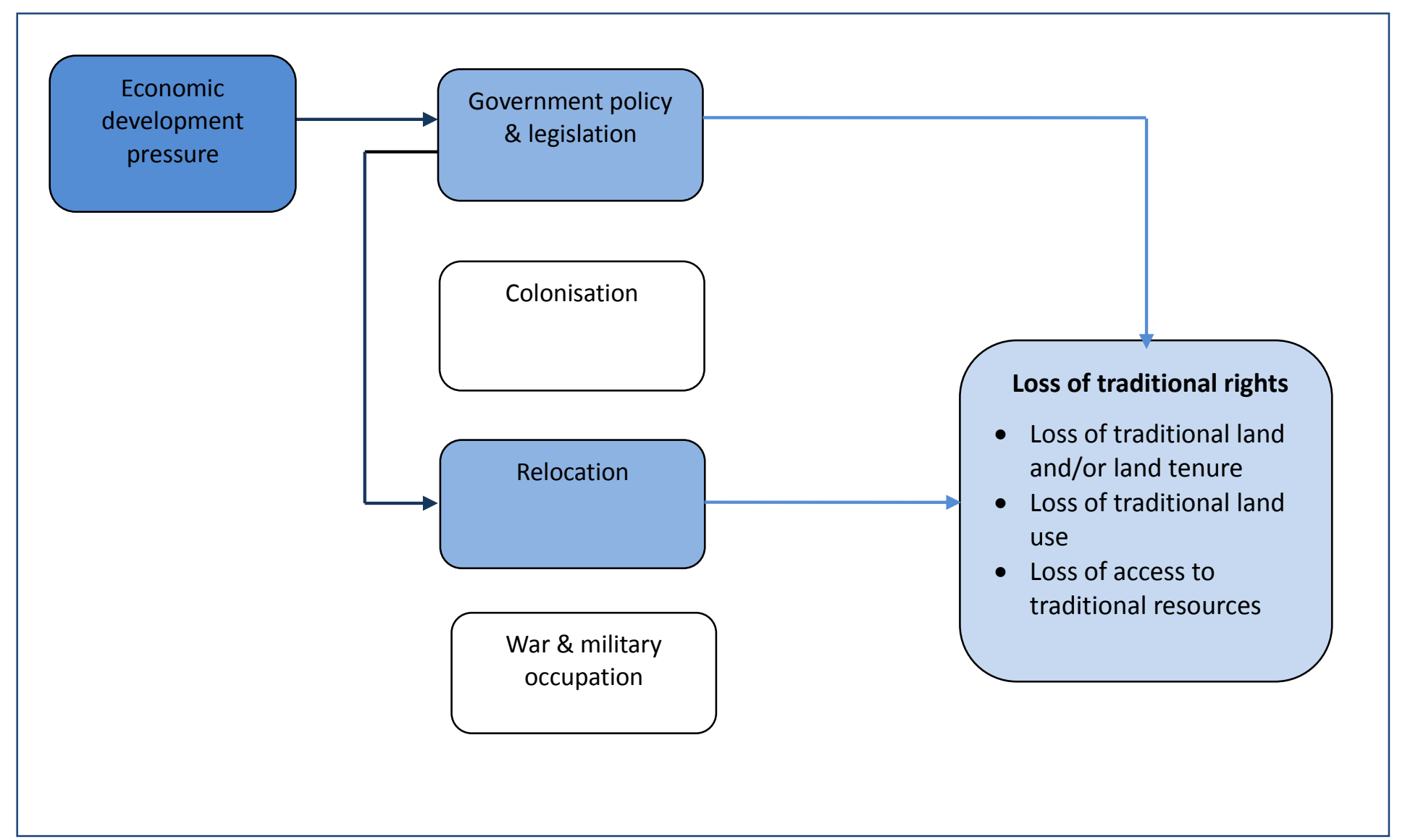

Figure 3.16 An example of the threat pathways of Loss of traditional rights 


\subsubsection{Threat pathways of loss of traditional institutions}

The loss of traditional institutions may be caused by government policy and legislation, colonisation, contact with other cultural groups, or war and military occupation; or any combination of these factors. In the meantime, government policy and legislation may be influenced by economic development pressure; and contact with other cultural groups may be triggered by government policy and legislation.

In this section, I will use the case study provided by Milton (2001) as an example. By focusing on indigenous forest communities in the Brazilian Amazon, Milton (2001) observes that along with the historical colonial influence and increased contact with non-indigenous groups, increasingly more indigenous peoples there, such as the Mayoruna people of Lobo Creek and Matis people of Itui, consider that speaking Portuguese is beneficial. As she illustrates, little by little, individuals in each village learn Portuguese, especially the young men. These young men then become the "voice of the village" when dealing with non-indigenous people; negotiating, translating and explaining. They generally achieve high status and considerable influence and power in village life. As Milton explains, "They also draw attention from the traditional wisdom of older generations (the village leaders); thereby diluting the latter's influence and disrupting the vertical transfer of traditional information from generation to generation" (Milton, 2001:294). This change of community leadership represents a gradual change from their traditional institutional arrangements. As long as the elders no longer hold power and influence in community decision-making, the decisions made by the younger leaders are more likely to reflect Westernised world views, rather than their traditional one.

While in many cases, the loss of traditional institutions is enforced by government policy and legislation, or by violent actions associated with war and military occupation, the change of traditional institutions has been a voluntary process in this case study. The change has been undertaken by the indigenous peoples under the influence of 
colonisation, and increased integration with other cultural groups. The implementation of the Westernised development model in the region may be the fundamental cause of the change. 


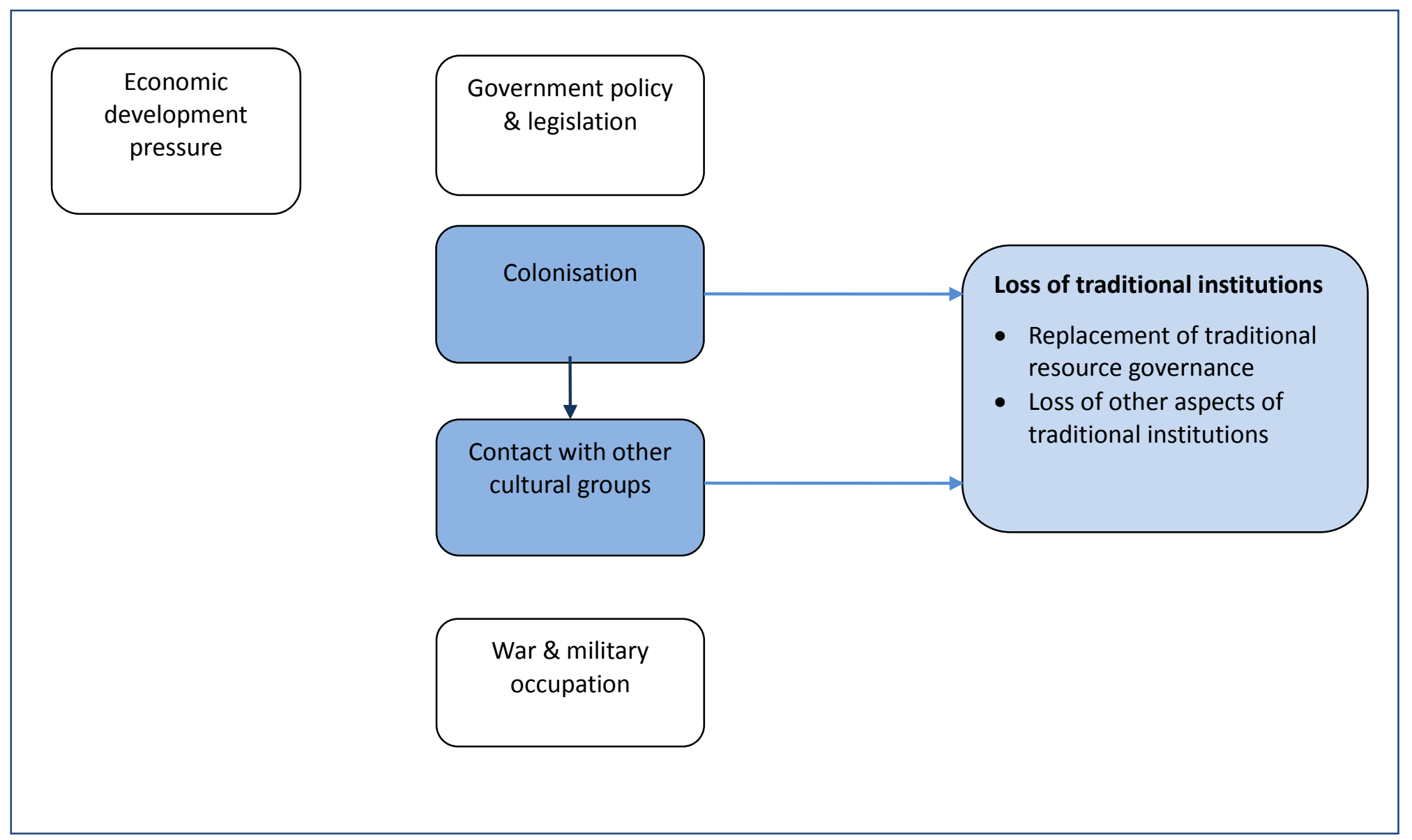

Figure 3.17 An example of the threat pathways of Loss of traditional institutions 
The above analysis indicates that there is a complex web of causality among direct threats and their underlying causes. To understand this web is critically important for planning effective and long-lasting TEK conservation actions. The long-term TEK conservation success would not be achieved unless both direct and underlying threats are addressed.

\subsection{Emerging themes from the TEK threat typology}

Through a detailed analysis of the TEK classification system and the causal web among TEK threats, several emergent themes have arisen. First, TEK is facing multiple threats across a range of institutional scales, from local and regional, to national and international levels. As Figure 3.11 demonstrates, direct threats often occur at local and regional levels and affect TEK at community level directly (e.g. change of traditional livelihood practices; change of traditional religion and beliefs; loss of traditional institutions). The first layer of underlying threats, however, can be both regional and national influences (e.g. indigenous population decline; marginalisation by dominant societies; government policy and legislation). The ultimate layer of underlying threats often derives from national (e.g. national government policy and legislation; national economic development pressure) and international levels (e.g. colonisation; economic globalisation; global migration). If we focus on individual case studies, this multi-scale causal web also exists. For example, in the case of the Tete Juan community in British Columbia in Canada, the community elder perceived the major direct threat to local TEK as the degradation of TEK transmission. He summarised that such degradation has been caused jointly by contact with other cultural groups at the local level, regional migration processes and colonisation. These underlying causes occur across local, regional and national levels simultaneously (see more details about the case in section 2.3.1.1). This cross-scale nature of TEK threats therefore requires TEK conservation action to be addressed at multiple scales.

Second, TEK threats are acting at different temporal scales; some involve rapid transitions while others may be slower. For example, in the case of relocated Mongolian herders in Alashan region (in Inner Mongolia of China), the government-forced relocation policy led directly to changes in their traditional livelihood and to the natural 
resources they traditionally lived upon (from pastoralism in a mountain area to agriculture in a lowland area). This transformation occurred rapidly between 1996 and 2000 (see more about the case in section 2.3.1.4; Tang and Gavin, 2010). On the contrary, in the case of the Bali people in Venezuela, the change of local livelihood activities and the loss of local ethnobotanical knowledge had been occurring alongside the influence of outside markets gradually over decades (Lizarradle, 1997, 2001) (see more details about this case in section 3.1.2). The time scales of various threats have important implications for informing conservation actions. They indicate which actions must be undertaken urgently to deal with rapid transitions, and which are more suitable for longer-term solutions (see more discussion about conservation action design in Chapter $4)$.

\section{Third, different threats may have different degrees of influence on TEK; some} threats have more immediate and localised impacts, while others are the underlying causes of many other threats. For instance, relocation may cause immediate changes of traditional TEK practices at community level. On the contrary, economic development pressure often affects TEK change through causing many other threats, such as government policy and legislation, and migration. The different degrees of influence also require different conservation design.

In a word, the core message produced from the TEK threat typology and the above analysis is that the diagnosis and treatment of TEK threats has to be carried out in a cross-scale, complex social-ecological system. The typology indicates that globally, indigenous peoples are confronting multiple challenges and threats that cover social, ecological, political and cultural domains. Thus, to deal with TEK threats and carry out conservation actions requires multi-disciplinary knowledge and skills. More importantly, it requires collaboration across a range of temporal, spatial and institutional scales (Berkes, 2010).

\subsection{Power relations and TEK threats}

The above analysis of the interconnectivity and causal relationships among TEK threats illustrates that TEK and its holders (usually the local community) are merely one actor 
in a highly complex and dynamic political-social-ecological system. In this system, however, the TEK holders often have less power than governmental and international sectors, such as international trade organisations (Banks, et al., 2003; Gragson and Blount, 1999; Hanna et al., 1996; Posey and Dutfield, 1996). The power relations between different layers of this complex social-ecological system, especially between the community level and upper (external) levels, have been a key subject regarding indigenous issues. A fundamental question about power is who is involved in decisionmaking? As many political ecologists point out, power relationships exert a core influence on sustainable resource management and community empowerment, and therefore on the conservation and practice of TEK. The unequal power relationships between authorities and local communities have been widely observed and reported.

This power imbalance makes the community and its TEK more vulnerable to outside influences, including political, economic, cultural and social influences (Aran et al., 2004; Berkes, 1998, 2007; Brodt, 2001; Florey, 2001). Let us take economic development pressure as an example. As Figure 3.11 shows, economic development pressure has been the root cause underlying many examples of TEK loss. Economic development pressures can lead to government policy and legislation, the influence of outside markets, and even colonisation. International competition has driven economic development to become a major focus of most state governments around the world. However, this economic development drive has also been reported to cause many social and political conflicts, especially in developing countries (e.g. Collings, 2009; Kendie and Guri, 2006; Smith et al., 2000). The sources of conflicts often relate back to competing visions of what development means and who gets to decide what form it should take (Smith et al., 2000). This is particularly true of questions about access to natural resources, land ownership and the importance of environmental stewardship (Collings, 2009). Conflicting development ideologies, rights, and ethical questions have been heavily debated in recent years. While the conventional, Western development model has spread out widely across the globe, more and more critiques are emerging from developing countries. For instance, as Kendie and Guri (2006: 334) state, "the results of the United Nations Development Decades and the various programmes of the World Bank and the International Monetary Fund, which are derived from the conventional development model in many developing countries, have been terribly disappointing [e.g. 
in Ghana]". This trend of realising the difficulties for reducing poverty and empowering indigenous people via the conventional development model, calls for alternative development approaches which involve more empowerment of people at the grassroots, self-reliance, sustainable development, respect for local culture and its traditional knowledge and wisdom, and an equal partnership with communities (Gohlert, 1993).

Many examples discussed in my analysis (e.g. Case et al., 2005; Cruz García, 2006; Hill, 2004; Lizarralde, 2001; Robert et al., 2004; Ross, 2002a; Zent, 1999; Zent and Maffi, 2009) demonstrate that the loss of TEK and TEK transmission in indigenous societies is largely caused by cultural influence from dominant societies and/or other cultural groups. As Case et al. (2005) point out, the Manus people in Papua New Guinea have been subject to Western culture for almost a century. Acculturation has taken place through the Churches' influence and by educational means. As a by-product of the acculturation process, traditional medicinal plant usage is being replaced with modern pharmaceuticals, even though Western medicine is rarely available. Case et al. therefore warn that the Manus are only one example of an indigenous group who have lost significant traditional knowledge and practices; and it is reasonable to speculate that, "globalisation with these repercussions is occurring in numerous locations of similar circumstances. Without significant efforts, the diversity of countless cultures will disappear just as the medicinal plant knowledge of the Manus has" (Case et al., 2005:364).

Many studies (e.g. Berkes, 2010; Brondzio et al., 2009; Lemos and Agrawal, 2006; Ostrom and Janssen, 2004) point out the necessity of power decentralisation in successful resource governance. However, the actual processes of power sharing and power devolution have remained challenging. The willingness to engage in and carry out the actions involved in power devolution from central government level has proven to be problematic across the globe (Bene, and Neiland, 2006; Berkes, 2009; Brown, 2002; Holt, 2005; Ribot, 2002). As some scholars suggest, the power of centralised management agencies should be redistributed and balanced, yet not eliminated (e.g. Berkes, 2010; 0’Brien et al., 2009; Stern, 2005). In any case centralised power has to be exercised carefully, because the local indigenous social organisation is often in a powerless position in terms of decision making, and vulnerable to the decisions that are 
made by dominant institutions. Most central governments maintain control over resource governance; they may either support or undermine lower level institutions, especially those that are community-based (Berkes, 2010).

\subsection{The potential user groups of the typology}

As discussed in section 3.2, because the threats exist at multiple levels, effective TEK conservation also needs to be carried out at multiple levels, by multiple actors simultaneously. The users of this typology need to include entities at various scales, from local to global. I have identified five user groups in particular.

\section{A. Indigenous communities and their organisations}

One key potential user of the typology is TEK holders - the indigenous communities. This is because the internal motivation and efforts toward conservation from inside of a community are probably the most efficient and long lasting driving force for community-based TEK conservation projects. This community-initiated or communitybased conservation approach has been widely recognised and recommended in the literature in recent years (e.g. Berkes, 2002, 2004, 2007; Gavin et al., 2007; Leach et al., 1999; Ostrom, 1990; Xu and Melick, 2007). By applying the typology, a community should be able to identify and target the problems that threaten their knowledge and practices. This clarification can raise the awareness of TEK threats and better enable communities to search for resolutions. The typology, as a unified classification system can also enable TEK holders to communicate across communities and share information.

\section{B. The academic and scientific community}

The academic and scientific community has served as a key voice regarding the status of TEK, in both the academic literature and in the international political arena. For this diverse community, the typology may be particularly useful to facilitate cross-project and cross-region communication, and to use in constructing common TEK databases. As I discussed earlier, most of the current TEK research concentrates on specific locations and specific communities. However, indigenous populations are increasingly confronting global challenges, which require rapid and cooperative conservation actions. The implementation of the typology in various locations and projects can actively 
facilitate the sharing of information and data, and the formation of common databases. The lack of comparable data and common databases has been a major obstacle to TEK research addressed by many researchers (e.g. Reyes-Garcia et al., 2005; Zent, 2001). Common databases will also be very useful in providing baseline data for researchers prior to their undertaking of specialised investigations (Zent and Maffi, 2009).

\section{Non-governmental organisations (NGOs)}

To location-based NGOs, the typology will provide a tool for examining TEK threats in a location, which is useful for prioritising resources and funds for organisational planning. By understanding local problems accurately, an NGO is more likely to optimise its function in a location. To international NGOs, the typology also offers a tool for comparable data analysis and data sharing.

\section{Governmental agencies}

For governments and their agencies, the typology provides a powerful tool for undertaking assessment of TEK status in the region or country and identifying the major TEK threats. It then enables the agencies to produce and implement conservation policies and measures, and guides the allocation of funds and other conservation resources.

\section{E. International policy organisations}

TEK degradation is a global issue. The TEK system and TEK holders are living in an inter-connected world, which faces many common threats globally, such as climate change, natural resource degradation and global market influences. In this globalised era, international policy organisations such as CBD, UNDP, IUCN, UNESCO and UNEP, play an increasingly important role in guiding national policy making and allocating international funds. Thus, the implementation of the typology is critically important to these organisations. By applying the typology, these organisations will be able to draw a global picture of TEK status and TEK threats. This should make it easier to identify and prioritise the most important TEK conservation hotspots where international funds should be allocated. These organisations will also be enabled by the typology to advise better on the TEK conservation efforts of national governments. 
To conclude, the degradation of TEK across the globe has been reported both in the literature $(n=152)$ and by the survey responses $(n=137)$. According to the survey results, this trend is most significant in Africa and Asia, where all the responses claimed TEK degradation. Responses from North America, Europe and Oceania claimed overall loss but also reported a few cases of TEK gain. This finding fills a geographic gap in the literature, where very limited studies in Oceania and no studies in Europe have previously been reported.

The emphasis of this research is to explore the causes of TEK degradation and put these causes into a systematic analysis. As a result, this research develops a typology of TEK threats, in which multiple levels of direct and underlying threats are comprehensively listed. The direct threats are the human activities that directly cause TEK degradation. It includes six categories: 1) loss of the pathways of TEK transmission; 2) change of traditional livelihood practices; 3) change of traditional religion and beliefs; 4) change of traditional environment and natural resources; 5) loss of traditional rights; and 6) loss of traditional institutions. Even though the survey and the literature identify these threats in different ratios, these threats require equal attention and conservation effort.

Underlying threats are the causal factors of direct threats. The underlying threats are categorised as: 1) government policy and legislation; 2) contact with other cultural groups; 3) influence of outside markets; 4) colonisation; 5) relocation; 6) marginalisation by dominant societies; 7) war and military occupation; 8) indigenous population decline; 9) migration; and 10) economic development pressure. These underlying causes cover social, political and cultural domains and function as an interconnected web.

In real-world cases, many or all of these threats act simultaneously. The causal relationships between threats are a complex system, in which multi-scale interactions exist and multiple organisations are involved. This system is determined by a dynamic and unbalanced power structure. In this power dynamic, as various TEK threat pathway analyses illustrate, TEK and its holders are often positioned powerlessly and passively. This disadvantageous position makes indigenous communities and their TEK more vulnerable to outside influences. As a key finding of this research, government policy 
and legislation have been widely perceived and reported to be responsible for TEK loss globally. This reflects the unequal power relations between communities and the authorities.

The production of a TEK typology fills an important gap in the TEK research field. As a unified classification system, it may enable TEK holders to identify the problems that threaten their traditional knowledge and practices, and motivate their search for solutions. It can facilitate academic and scientific communities to carry out cross-project and cross-region communication, and enable the production of comparable results despite their different disciplinary backgrounds and locations. The typology can also provide a tool for developing a global framework of TEK monitoring and conservation, and common databases for information sharing. It therefore may be able to guide government and international agencies to produce and implement TEK conservation measures, and to guide the allocation of fund and other resources towards TEK conservation. 


\section{CHAPTER FOUR}

\section{TEK CONSERVATION ACTIONS}

\section{INTRODUCTION}

The values of TEK have been discussed in Chapter One in detail. In summary, TEK plays an important role in the livelihoods, well-being and cultural identity of indigenous and local people, and can facilitate indigenous empowerment. It can also inform contemporary science, resource management and conservation with practical knowledge and experience which has evolved over generations. In Chapter Three, I presented the evidence of trends in TEK loss worldwide with figures and examples. The value of TEK and the declining trend of TEK both contribute to the urgency of TEK conservation. As I discussed in the previous chapter, TEK change is driven by a complex web of direct and underlying threats, which occur at multiple temporal and spatial scales. The study of TEK threats allows us to target conservation actions and may assist in the design of interventions. In this chapter, I focus on mechanisms which have been applied or suggested to protect TEK and discuss the design and implementation of a multi-scalar system of TEK conservation.

\subsection{The international framework for TEK conservation}

The protection of TEK has been a feature of international agreements since the early 1990s. In the last two decades, a number of international agencies, such as the United Nations (UN), United Nations Educational, Scientific and Cultural Organisation (UNESCO), United Nations Environmental Programme (UNEP), and International Union for Conservation of Nature (IUCN), have developed a range of policies aimed at the protection of indigenous rights and traditional knowledge. The UN Declaration on the Rights of Indigenous People (United Nations, 2007: Article 31) provides the most explicit recognition internationally of indigenous people's rights to their traditional knowledge:

Indigenous peoples have the right to maintain, control, protect and develop their cultural heritage, traditional knowledge and traditional cultural expressions, as 
well as the manifestations of their sciences, technologies and cultures, including human and genetic resources, seeds, medicines, knowledge of the properties of fauna and flora, oral traditions, literatures, designs, sports and traditional games and visual and performing arts. They also have the right to maintain, control, protect, and develop their intellectual property over such cultural heritage, traditional knowledge, and traditional cultural expressions.

The Convention on Biological Diversity (CBD, 2006) constructs specific measures for recognising and protecting TEK. Article 8(j) of the Convention promotes participant countries to:

... respect, preserve and maintain knowledge, innovations and practices of Indigenous and local communities embodying traditional lifestyles relevant for the conservation and sustainable use of biological diversity and promote their wider application with the approval and involvement of the holders of such knowledge, innovations and practices and encourage the equitable sharing of the benefits arising from the utilisation of such knowledge, innovations and practices.

Article 8(j) also constructs a fundamental principle for TEK protection that the use of indigenous traditional knowledge, innovations and practices should only occur with the approval and involvement of the indigenous or local community. More importantly, any benefits that arise from its use should be shared with the people or community from which that knowledge originated (CBD, 2006).

Apart from the Declaration on the Rights of Indigenous Peoples and the CBD, there are numerous other international policies and agreements that address the importance of, and urge the action towards TEK conservation (see Table 4.1 below). For example, the International Labour Organisation Convention No. 169 (ILO, 1989: Article 2 \& 23) includes several provisions regarding the need to respect and protect "customs and traditions", as well as "handicrafts, rural and community-based industries and traditional activities". 
Table 4.1 Summary of Major International Instruments that Recognise Indigenous Peoples' Right to Protect their Traditional Knowledge

\begin{tabular}{|l|l|c|}
\hline \multicolumn{1}{|c|}{ International Policy Instruments } & \multicolumn{1}{c|}{ Provision } & Year \\
\hline Agenda 21 & Paragraph 26.1 & 1992 \\
\hline The Convention on Biological Diversity & Article 8 (j) & 1992 \\
\hline The Declaration on the Rights of Indigenous Peoples & Articles 11 \& 31 & 2008 \\
\hline $\begin{array}{l}\text { The Rio Declaration on Environment and } \\
\text { Development }\end{array}$ & Principle 22 & 1992 \\
\hline $\begin{array}{l}\text { The Universal Declaration of Human Rights } \\
\text { The International Covenant on Economic, Social and } \\
\text { Cultural Rights }\end{array}$ & $\begin{array}{l}\text { Article 15, paragraph } \\
1 \text { (c) }\end{array}$ & 1966 \\
\hline $\begin{array}{l}\text { The International Covenant on Civil and Political } \\
\text { Rights }\end{array}$ & Article 27 & 1966 \\
\hline $\begin{array}{l}\text { The International Labour Organisation Convention } \\
\text { No.169 concerning Indigenous and Tribal Peoples in } \\
\text { Independent Countries }\end{array}$ & $\begin{array}{l}\text { Articles 2, 13, 15 \& } \\
23\end{array}$ & 1989 \\
\hline
\end{tabular}

(Source: Australian Human Rights Commission, 2008)

The majority of the countries around the world have agreed on these different mechanisms related to TEK conservation. For instance, by the end of 2011, 168 countries had officially ratified the CBD (CBD, 2012). This reflects that the agreement of biodiversity protection, in which TEK and its protection is included, has become a trend around the globe. While the necessity of conserving TEK is no longer debatable in the literature and among most countries, the big question now is what we can do for conserving TEK before it is too late? Policies that intend to protect and revitalise TEK have been produced at the international level. However, how to transform these policies and measures into actions, especially at national and regional levels has remained a major challenge. As Collings (2009: 108) points out, many decisions made at the international level have not always been respected or implemented at the national level, and "indigenous peoples' voices are all too often marginalised, if heard at all". Thus, the most urgent need in TEK and indigenous rights conservation field is to develop a practical action strategy and implement it at the ground level. 


\subsection{Why is this study important?}

This research is the first study to identify all available conservation mechanisms, including ones already in use in the field, as well as potential ones that to date have only been discussed in the literature. By studying and synthesising all the applied and potential conservation options, this research develops a standard classification system of TEK conservation actions. This represents a powerful toolbox for conservation project design. By applying this typology, conservation actors, including TEK holders, practitioners, researchers, NGOs and governmental and international agencies, can choose appropriate conservation mechanisms that suit their local context and match their capacity.

As discussed in the previous chapter, to date, most of the TEK studies are locationspecific case studies, and have been focusing on exploring, recording and examining TEK. None of the studies I have reviewed $(n=152)$ specifically focuses on TEK conservation options or actions. Within these studies, 79\% (n=120) of them suggest immediate actions for TEK conservation without recommending any specific actions. While there are 35 studies (23\%) which recommend specific actions for TEK conservation, only 17 (11\%) studies provide examples of TEK conservation actions that have been undertaken in the field. Even though a number of international policies, agreements and conventions have made a strong voice to advocate for indigenous rights protection and TEK conservation, documented conservation actions have been limited. Therefore, this chapter seeks to address several critical literature gaps related to TEK conservation: a review of available TEK conservation actions, development of a standard classification system for TEK conservation options, and principles for designing effective TEK conservation.

It is also important to note that, within the TEK studies I have reviewed, none of them focuses on the assessment of TEK conservation outcomes. The study and implementation of TEK conservation still remains at an experimental stage, and the field lacks an appropriate monitoring and evaluating system. Without proper assessment, it is difficult to know which TEK threats have been addressed, and what barriers obstruct the efficiency of conservation actions (Lee, 1999). Based on the perceptions of survey respondents and interviewees, this research will provide a discussion of the triggers and 
barriers to TEK conservation success. I will also utilise survey responses and literature review (see discussion of the methods in Chapter 2) to develop conservation action design principles. This work represents an important baseline on which future research and analysis can be further developed.

\subsection{Aim and objectives}

The aims of this chapter are to explore the TEK conservation options that have been undertaken in the field and are suggested in the literature, and to develop a typology of TEK conservation actions as a toolbox for TEK holders and practitioners.

There are four key objectives:

1. To examine the applied and potential TEK conservation options.

2. To develop a TEK conservation typology.

3. To assess perceived triggers and barriers to TEK conservation.

4. To develop the principles for TEK conservation design.

\section{RESULTS}

As in Chapter Three, the results in this chapter are drawn from two methods: 1) the literature review $(n=152)$ provides primary understanding and overview of TEK conservation options addressed by scholars; and 2) the global survey ( $n=137)$ and its follow-up interviews $(n=46)$ identifies the TEK conservation actions in use addressed by TEK holders and practitioners, and forms the foundation of the TEK conservation typology (see more details about the research methods in Chapter 2, section 3.1, 4.1 \& 5). In this section, I will first present the TEK conservation action typology and analyse each conservation option; then discuss geographic patterns of TEK conservation actions and link them to the global distribution of TEK threats; and finally discuss the triggers and barriers, and design principles for the conservation success. 


\subsection{The Typology of TEK conservation actions}

As defined in Chapter Three (section 1.3), conservation actions are the actions that undertaken by TEK holders and/or practitioners with the target of conserving and revitalising TEK, and empowering TEK holders when possible. The typology covers both conservation options currently in use ${ }^{29}$, and the potential conservation options discussed in the literature. Please note that the development of this classification system targets to cover all possible actions towards TEK conservation, including the actions directly targeting the aspects of TEK (e.g. TEK documentation), and the actions targeting broader natural and cultural conservation, and political support towards the indigenous community that may contribute to TEK conservation (e.g. indigenous capacity building).

${ }^{29}$ The data collected in the survey were answers to the question of "what has been carried out to conserve TEK in your locations?" 
Table 4.2 Typology of TEK Conservation Actions

\begin{tabular}{|c|c|c|}
\hline Category & Definition/explanation & Example \\
\hline $1.1 \underline{\text { Institutional development }}$ & \multicolumn{2}{|c|}{$\begin{array}{l}\text { The strengthening of indigenous social cohesion and social organisation, the conservation or re-establishment of } \\
\text { traditional norms and rules about resource use and management }\end{array}$} \\
\hline 1.1.1 Self-government & $\begin{array}{l}\text { The establishment and development of } \\
\text { autonomous governance which is organised } \\
\text { and led by the indigenous groups }\end{array}$ & $\begin{array}{l}\text { Political independence and self-governance achieved in Cook } \\
\text { Islands, Fiji and Vanuatu between the 1960s and the } 1980 \text { s }\end{array}$ \\
\hline 1.1.2 Self-organisation & $\begin{array}{l}\text { The establishment and development of } \\
\text { organisations organised by indigenous groups } \\
\text { with a focus on promoting traditional } \\
\text { practices, traditional culture and indigenous } \\
\text { empowerment }\end{array}$ & $\begin{array}{l}\text { An organisation formed in southwest Yukon Territory, Canada with } \\
\text { an aim to promote the use of traditional medicine, organic } \\
\text { agriculture, general conservation techniques, and defend } \\
\text { indigenous land rights }\end{array}$ \\
\hline $\begin{array}{l}\text { 1.2.2 Partnership with external } \\
\text { parties }\end{array}$ & $\begin{array}{l}\text { A partnership that developed between } \\
\text { indigenous community/ies and governmental } \\
\text { agencies, research institutions and/or other } \\
\text { external organisations with a target of } \\
\text { conserving TEK and traditional culture }\end{array}$ & $\begin{array}{l}\text { Co-management between Inuit communities and regional } \\
\text { government in Nunavut Territory, Canada; } \\
\text { Participatory research relationship between local Maori iwi and } \\
\text { research institute in New Zealand }\end{array}$ \\
\hline
\end{tabular}




\begin{tabular}{|c|c|c|}
\hline 1.3 Indigenous financing & \multicolumn{2}{|c|}{$\begin{array}{l}\text { Advancing the ability of fundraising and fund management by indigenous communities regarding TEK } \\
\text { conservation and indigenous empowerment. The funding sources may include governmental agencies, private } \\
\text { foundations and international agencies }\end{array}$} \\
\hline $\begin{array}{l}\text { 2.1 Traditional lifeways } \\
\text { programmes }\end{array}$ & $\begin{array}{l}\text { Indigenous community-based programmes } \\
\text { that aim to revitalise or promote traditional } \\
\text { lifestyles }\end{array}$ & Elder camps and youth challenge in Winnipeg, Manitoba, Canada \\
\hline $\begin{array}{l}\text { 2.2 Environmental } \\
\text { conservation activities }\end{array}$ & \multicolumn{2}{|c|}{$\begin{array}{l}\text { Indigenous community-based projects that aim to conserve biodiversity and natural resources, in which TEK is } \\
\text { protected and TEK holders are involved }\end{array}$} \\
\hline $\begin{array}{l}\text { 2.2.1 Community-initiated } \\
\text { environmental conservation } \\
\text { programmes }\end{array}$ & $\begin{array}{l}\text { Conservation programmes initiated and led } \\
\text { by indigenous communities }\end{array}$ & $\begin{array}{l}\text { Heritage seed program in Cherokee, North Carolina, USA; } \\
\text { Saxoul tree conservation project initiated and managed by local } \\
\text { community in Alashan region, Inner Mongolia, China }\end{array}$ \\
\hline 2.3 TEK commoditisation & \multicolumn{2}{|c|}{$\begin{array}{l}\text { The transformation of aspects of TEK, which are not normally regarded as having monetary value, into } \\
\text { commodities }\end{array}$} \\
\hline $\begin{array}{l}\text { 2.3.1 Ethno- tourism or eco- } \\
\text { cultural tourism }\end{array}$ & $\begin{array}{l}\text { Tourism oriented towards indigenous culture, } \\
\text { local landscapes/seascapes, or local species }\end{array}$ & $\begin{array}{l}\text { Ethno-tourism enterprises showcasing traditional song, dance and } \\
\text { costume in Shuar territory, Morona-Santiago Province, Ecuador }\end{array}$ \\
\hline
\end{tabular}

30 This category includes a wide range of activities, from hiring indigenous park rangers to co-management programmes. 


\begin{tabular}{|c|c|c|}
\hline $\begin{array}{l}\text { 2.3.2 Indigenous products } \\
\text { trading }\end{array}$ & $\begin{array}{l}\text { Small scale trading activities of indigenous } \\
\text { products that earns cash for the community } \\
\text { and facilitates TEK transmission }\end{array}$ & $\begin{array}{l}\text { Revival of aspects of traditional back strap loom weaving using } \\
\text { natural dyes by women with new coop marketing outlets in } \\
\text { Tenejapa, Chiapas, Mexico }\end{array}$ \\
\hline 3. Education and awareness & \multicolumn{2}{|c|}{ Conserving and promoting TEK through educational systems and public awareness raising } \\
\hline $\begin{array}{l}\text { 3.1 Include TEK in formal } \\
\text { education }\end{array}$ & \multicolumn{2}{|c|}{ To advance TEK transmission through encompassing TEK and indigenous languages in formal education system } \\
\hline $\begin{array}{l}\text { 3.1.2 Include TEK in school } \\
\text { curricula }\end{array}$ & $\begin{array}{l}\text { To teach the contents of TEK in order to } \\
\text { enhance students' knowledge of and interests } \\
\text { in TEK and indigenous culture }\end{array}$ & $\begin{array}{l}\text { Guidebook of local plants and animals is included in school } \\
\text { curriculum in Pikangikum, Ontario, Canada }\end{array}$ \\
\hline$\underline{\text { 3.3 Indigenous media and }}$ & \multicolumn{2}{|c|}{$\begin{array}{l}\text { Raising awareness of TEK and its values amongst indigenous communities and general public, through } \\
\text { communication strategies and informal learning processes }\end{array}$} \\
\hline 3.3.1 Indigenous media & $\begin{array}{l}\text { Raising awareness and recognition of TEK and } \\
\text { other indigenous traditions by applying } \\
\text { communication tools, such as newsletter, } \\
\text { radio station and website }\end{array}$ & $\begin{array}{l}\text { Community newsletter in New Zealand; } \\
\text { Community radio programmes in Pikangikum, Ontario, Canada, } \\
\text { organised by the local First Nation }\end{array}$ \\
\hline
\end{tabular}




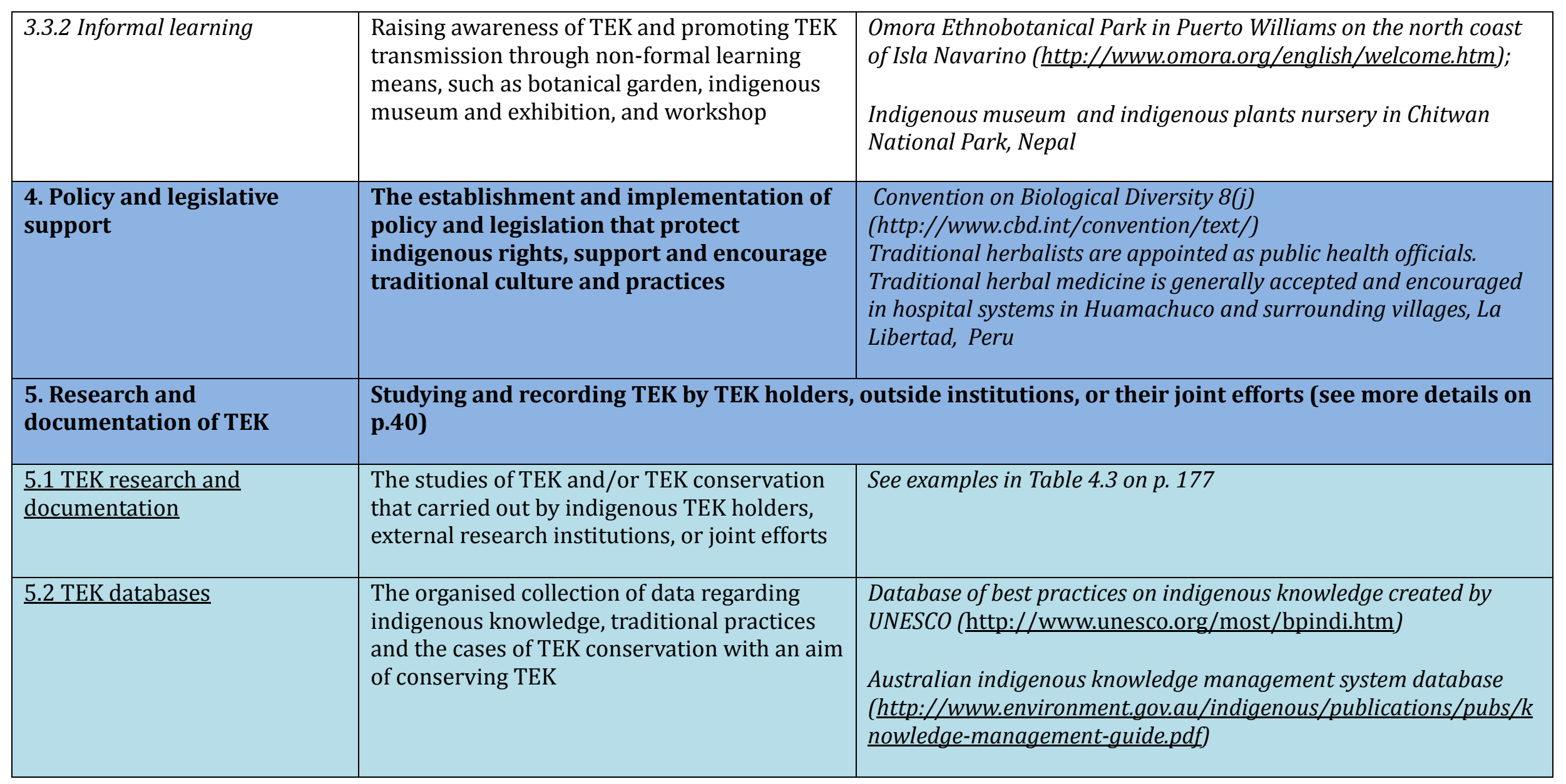




\subsection{The conservation options}

The majority ( $82 \%, \mathrm{n}=112)$ of the survey respondents claimed that at least one conservation action had been undertaken in their focal sites. The three most frequently listed conservation options are research and documentation $(39 \%, n=74)$, community-based TEK conservation activities (in situ) $(25 \%, \mathrm{n}=48)$, and education and awareness $(16 \%, n=30)$. Apart from these three, Capacity building was listed by $12 \%$ $(n=23)$ of the responses, and Policy and legislative support was listed by $8 \%(n=15)$ of the responses. Please note that, because the survey question of "What have been done to conserve TEK in your location?" was open end, multiple answers could be given. Thus, the total number of the positive responses is 190 . The percentage of various conservation options was calculated based on this figure. Below, I analyse the five categories listed in the typology with examples from the survey and its follow-up interviews, as well as literature review.

\subsubsection{Action One - Capacity building}

Capacity building refers to a long-term, ongoing process in which indigenous communities build or improve their collective resources and skills to conserve and/or revitalise their traditional culture and lifestyles. This conception of indigenous capacity building draws from two decades of discussion in international literature and policy around community capacity building and engagement (King and Cruickshank, 2012). The earliest references to capacity building in the literature date from the early 1990s in the works of UNCED (1992), Agenda 21 (1992), and the UN Commission on Sustainable Development (UNDP, 1991). UNCED (1992: 232) suggests that capacity building "encompasses human, scientific, technological, organisational, institutional and resource capacities". Many scholars argue that bottom-up, locally based approaches permit policy to be more socially inclusive and help ensure the social stability and cohesion without constricting economic growth and structural adjustment (Mannion, 1996; Popham, 1996; Schuftan, 1996). Such approaches require a process that builds on local strengths and promotes community participation and leadership, as well as ownership of both the problems and the solutions by indigenous peoples (Simpson et al, 2003). They also argue that the basis for sustainable bottom-up community development lies in community capacity building (e.g. Cavaye, 1999; Labonte, 1999). 
Capacity building therefore is a driver for communities to take responsibility for their own development, to cease relying on government for direction and solutions. There is a popular argument that if communities can survive through economic and social crises, they can best do so "by becoming empowered, by building their existing capacity and by using the skills they have to make their own futures" (Simpson et al, 2003:227).

2.2.1.1 Institutional development refers to the strengthening of indigenous social cohesion and social organisation, and the conservation or re-establishment of traditional norms and rules regarding resource use and management. The importance of the institutions involved in resource management at the local level has been discussed and highlighted in the literature from various disciplines, such as natural resource management (especially the commons management), community development, political ecology and conservation biology (e.g. Agrawal and Gibson, 1999; Agrawal and Ostrom, 2001; Berkes, 2010; Colchester, 2000; Ostrom, 1990, 2005, 2009; Ostrom and Janssen, 2004; Pahl-Wostl, 2009; Young, 2002). As Agrawal and Ostrom (2001) demonstrate in response to the performance deficiencies of governmental agencies in resource management, policies of devolving management responsibilities from the state to resource users have become increasingly widespread. The success of these decentralisation policies depends upon the local institutions and local capacity for taking collective actions.

Indigenous institutional development has also been considered a key component of steps towards self-determination ${ }^{31}$. To date, there are some national constitutions that recognise the right of self-determination of indigenous peoples (e.g. the Constitutions of the Philippines recognises the rights of Muslim and Cordillera peoples to selfdetermination in the form of autonomy; Daoas, 1995), whereas some countries avoid it (e.g. Chile's congress has voted against several initiatives that would constitutionally recognise the country's indigenous peoples as such; Stavenhagen, 2006). While it is important to have a substantial international and national legislative framework to legally recognise indigenous rights to self-determination, the institutional capacity of the indigenous societies to undertake such motion is equally essential. This may include

\footnotetext{
${ }^{31}$ Self-determination refers to the right of a group or nation to freely determine their political status and freely pursue their economic, social and cultural development without influence from other parties.
} 
knowledge of relevant policy and legislation, the capacity for presenting and claiming their rights in front of international agencies and national authorities, and more importantly, the capacity of self-determination (Kampel and MacKay, 1999).

Two approaches for institutional development have been addressed by the survey responses - self-government and self-organisation.

A. Self-government is the establishment and development of autonomous governance organised and led by the indigenous groups. Self-government is an important measure of self-determination processes. Over the last century, indigenous peoples have sought to maintain or regain the right to their self-government as a basic part of the fundamental freedoms that international law accords to all peoples (Stavehagen, 2006). Over years, through negotiations and treaties, constitutional reform or special legislation, indigenous peoples have been able in numerous instances to establish agreements with states regarding this right to self-government. For instance, many pacific island countries have successfully achieved independence and self-government alongside the decolonisation process in the last few decades, such as Cook Islands in 1965, Fiji in 1970 and Vanuatu in 1980 (Bertram, 1987; Gerry, 1996; Hoadley, 1992; Kloff, 1965). The indigenous peoples in these countries are in charge of determining the political structure, social and economic development and cultural development of the country.

In other countries which have colonial histories, there has been a process of increased indigenous presentation in government. For example, in Canada, the First Nations (also referred to as Aboriginal People in Canada) are actively involved in national political life and the local decision making system through its 630 governments and branches (Welch, pers. comm., 2011). A researcher (surv. resp., 2011) who works in southwest Yukon Territory, Canada described:

"Many Yukon First Nations are involved in co-management with the federal and territorial governments, and with public co-management boards. They are also developing TEK policy and databases ... In the Yukon, accumulated selfgovernment experience and self-organised activities by First Nations are enhancing the intergenerational transmission of knowledge and ensuring 
cultural adaptiveness in a rapidly changing landscape."

Similarly, the Tamsaling Nepal National Party, which represents the indigenous population in Nepal, has earned significant support from indigenous groups since being established in 2008. Indeed, it has now claimed its status as an independent political party in Nepal:

"The Tamsaling Nepal National Party is a national political party lead by Mr. Parshuram Tamang, former vice-chair of UNPFFII ${ }^{32}$...This Party was formed just four years ago. We do not have constitutional assembly members, however we are getting good supports from the indigenous and disadvantaged people. Most of the party members are from Tamang community. So some people say this is Tamang peoples' party, but in fact, we are following democratic norms and values and involve all community people" (Lama, pers. comm., 2012).

The above two examples demonstrate the increasing possibility of indigenous selfgovernment if their rights are respected and recognised by the state government and the national laws. However, in many other cases, this has not been possible, and national-level or regional-level government units still take it upon themselves to administer the affairs of indigenous communities (DESA, 2009). Recognising such issues, the Declaration on the Rights of Indigenous Peoples (2008) states in Article 33:

Indigenous peoples have the right to promote, develop and maintain their institutional structures and their distinctive juridical customs, traditions, procedures and practices, in accordance with internationally recognised human rights standards

B. Self-organisation refers to the establishment and development of organisations organised by indigenous groups with a focus on promoting traditional practices, traditional culture and indigenous empowerment. These organisations are usually initiated by indigenous communities, sometimes assisted by external organisations. These organisations can have various aims, such as general indigenous rights and empowerment, natural resource and biodiversity conservation, and cultural preservation.

32 UNPFII - United Nations Permanent Forum on Indigenous Issues 
Indigenous self-organisation has made considerable progress in many regions over the last 20 to 30 years. From the local level to the regional, national, and international levels, indigenous peoples' associations have become social and political actors in their own right, as witnessed by their continuing participation in the yearly sessions of the Working Group on Indigenous Peoples (WGIP) (Stavenhagen, 2005). For example, studies at various locations in Mexico found that organisations of indigenous people for various purposes have grown strongly in the last 20 years. Many of them are focused on traditional medicine, but also on organic agriculture, general conservation or defence of land rights (surv. resp., 2010). In Loreto, Peru, a researcher (surv. resp., 2011) witnessed the establishment of an indigenous Maijuna federation - FECONAMAI, as the response to threats to Maijuna biocultural resources. Maijuna elders and leaders established the Federation, which is representing all four Maijuna communities. The principal goals of the Federation are to "conserve the environment, conserve the Maijuna culture, and improve Maijuna community organisation". In short, the federation is fighting for the survival of the Maijuna on a daily basis (see more details about the Federation on http://www.rainforestconservation.org/archives/1156).

These indigenous organisations speak with many voices, but on the fundamental issues of their human rights, their objectives and their aspirations are often in remarkable agreement (DESA, 2009). In some countries they are now recognised as legitimate partners and interlocutors of governments and other social sectors on the national scene. In other countries the process has been more difficult; indigenous organisations may not be officially recognised, and their human rights to free association may not be completely respected (Dorough, 2009). To the extent that the rights of indigenous people themselves are sometimes neglected and ignored within existing power structures, their organisations and other human rights advocacy may also become victims of abuses and be denied under the law (Stavenhagen, 2007).

2.2.1.2 Alliance and partnership development is the formation and development of a network in which indigenous communities and external parties participate, in order to advance participating parties' common goals and interests in TEK. Living in a more and more globalised world, indigenous peoples are surrounded by dominant societies. It is 
often hard for one indigenous group to maintain and improve their indigenous rights without any collaboration with other indigenous groups or external organisations or without any support from governmental agencies at multiple levels (Berkes, 2007). This is because one group or one community usually lacks the capacity and the power of designing and implementing the best practices, especially when it faces the threats, challenges and opportunities occurring at scales beyond the local level. This situation therefore requires responses which are not just local, but cross-scalar. As Brondizio et al. (2009) point out, the multi-level nature of the resource use and management issues requires horizontal and vertical linkages among all actors. By using an example of institutional development of indigenous park management in the Xingu Indigenous Park in Brazil, the authors also highlight that one of the crucial horizontal strategies is "an expansion of the indigenous territory through alliance and support to other ethnic groups claiming land rights and demarcation in areas contiguous to the park and related watershed tributaries" (Bronzidio et al., 2009: 257). Likewise, in many other studies, the collaborative partnership and network between actors and across-scale has been addressed as a critical component of indigenous capacity building (e.g. Ostrom, 1990, 2005; Young, 2002a, 2002b).

A. Community alliances refer to networks that formed among indigenous communities with the common target of conserving nature and traditional culture and practices. Community alliances are usually initiated by community members, who share common interests in maintaining traditional practices and protecting or regaining indigenous rights.

A well-known community alliance is the International Alliance of the Indigenous-Tribal Peoples of the Tropical Forests (hereafter the Alliance). It is a worldwide network of organisations representing indigenous and tribal people living in tropical forest regions, including Africa, the Asia-Pacific and the Americans. The Alliance was established in 1992 during an indigenous conference in Malaysia. The objectives of the Alliance include: promoting full recognition of the rights and territories of indigenous and tribal peoples; promoting the development of indigenous and tribal peoples and their participation in decision and policy making; establishing effective networks between indigenous peoples at regional and international levels; and exchanging information 
and experiences to empower Alliance members to advocate for the rights of indigenous and tribal peoples (http://www.international-alliance.org/about whoweare eng.html, 2012). The Alliance has actively carried out regional capacity building and training programmes, as well as monitoring and participating in international policy processes such as the CBD article (8(j), the Action Plan of the World Summit on Sustainable Development (WSSD) and the UN Working Group on Indigenous Populations (WGIP).

There are also many smaller community alliance organisations formed in different regions with different focuses. For instance, in the territory of the Piaroa in the Amazonas State of Venezuela, agricultural cooperatives have formed across communities. The cooperatives encourage traditional honey harvesting, and Inga edulis plantation. These cooperatives support indigenous groups to cope with the pressure generated by commercial agriculture of modern crops (Heckler, surv resp., 2010).

B. Partnership with external parties refers to the partnership developed between indigenous communities and governmental agencies, research institutions and/or other external organisations with the common target of conserving traditional culture and practices. Co-management is a typical example of partnership between indigenous communities and governmental agencies. As Berkes et al. (2000) define, comanagement is a participatory process that provides a forum for rule making, conflict management, power sharing, leadership, dialogue, decision making, negotiation, knowledge generation and sharing, learning, and development among resource users, stakeholders, and government. Successful cases of co-management have been claimed by a significant number of researchers from both the survey and the literature (e.g. Berkes, 2002, 2010; Binder and Hanbidge, 1993; Nakashima, 1993; Usher, 1993). For example, by studying aboriginal population in southwest Yukon Territory and Nunavut Territory, Canada, a researcher (surv. resp. 2010) claimed that, "In both sites, the settlement of Aboriginal land claims has given substantive weight and power to collection and use of TEK in co-management decision processes. ... In the Nunavut Territory, the Nunavut Land Claims Agreement came into effect in 1993. The Nunavut Wildlife Management Board then was established based on the Agreement. The Board is comprised by nine members, four Inuit representatives, four government representatives, and a chairperson nominated by the Board. While it recognises that 
government has the ultimate responsibility for wildlife management, Inuit fishers had a chance to participate in research and Wildlife Harvest Study, as well as to use their traditional knowledge to develop management plans".

Another researcher (surv. resp., 2010) who studied thirteen villages in Panama and Darien provinces of Panama also addressed that, "There are projects in which NGOs and local indigenous people work together. Under the indigenous world-visions, the projects are targeting the local knowledge of natural resources (e.g. game and fish species, plants) and constructing plans of co-management".

The above examples illustrate the potential for cooperation with external agencies to strengthen the capacity of indigenous. Cooperation and partnership with external bodies can provide diverse functions and services to indigenous communities, such as raising start-up funds, institutional building, business networking and marketing, innovation and knowledge transfer, and technical training (Berkes and Adhikari, 2006). These services and support therefore are likely to facilitate community capacity building (Berkes and Adhikari, 2006). Based on ten Equator Initiative projects, Seixas and Berkes (2010) find that, successful projects tend to interact with a large array of support groups (typically, 10-15 partners). These partners included local and national NGOs; local, regional and national governments; international donor agencies and other organisations; and university and research centres. They emphasise that a diverse variety of partners are required to satisfy a diversity of community needs, and highlight the importance of networks and support groups in the evolution of community institutions (Seixas and Berkes, 2010).

2.2.1.3 Indigenous finance refers to advancing the ability of fundraising and fund management by indigenous communities regarding TEK conservation and indigenous empowerment. Although financial capacity is vitally important to any indigenous community and their projects, this conservation action was only mentioned by one informant and is absent from the literature. A sustainable future requires a sustainable fund source to support it. To develop indigenous financial capacity, it is vital to scope the potential fund sources. For an indigenous community, there are three main sources of donors: local and national governmental agencies, private foundations and international 
agencies.

Living in this globalised world, indigenous populations have much more contact with outside world than ever before. Even though, this contact can generate pressure and negative effects on TEK's survival, it also offers opportunities for gaining support and generating benefits for indigenous populations from the outside world (Berkes, 2007). For example, many international agencies, such as UNDP33, $\mathrm{CBD}^{34}$ and $\mathrm{IUCN}^{35}$ and $\mathrm{WWF}^{36}$ are supporting indigenous projects. They all provide a large amount of funding across the globe. Theoretically, all indigenous communities have an equal chance to gain this kind of international financial support. Even though, practically, there are huge differences in accessing ability from different communities due to various reasons, such as geographical location and political status. For instance, the international fund has been highly concentrated on Amazon and Africa over the last two decade, while Asian and Oceanic countries have had less attention (Berkes, pers. comm., 2011; Wu and Boggess, 1999).

While accessing fund sources is critically important, how to manage funds sustainably is another central issue of indigenous finance. A community-based integrated conservation and development project in the Alashan region (in Inner Mongolia of China) provides a good example here. This community-initiated project created its own project management committee though community election process. An accountant was elected for managing the community project finance. The initial project fund was from two major sources: community member's investment and a donor organisation. The daily decision of fund use was decided by the committee, any big decisions, on the other hand, was discussed and decided on the community meetings. The accountant reported to the committee once a month, to the community meetings once every three months, and to the donor organisation once every six months. This fund management system is based on the traditional community trust and social cohesion, as well as some modern financial management techniques (Tang and Gavin, 2010).

\footnotetext{
33 UNDP - United Nations Development Programme

${ }^{34}$ CBD - The Convention of Biological Diversity

35 IUCN - International Union for Conservation of Nature

36 WWF - World Wildlife Fund
} 
Information sharing is also important for strengthening indigenous financial capacity. This is because the modern financing is a relatively new territory for many indigenous groups (Berkes, pers. comm., 2011). The sharing of fund source information and fund management experience will shorten the learning curve, and avoid repeating failures.

\subsubsection{Action Two - Community-based TEK conservation activities}

Community-based TEK conservation activities refer to the conservation actions undertaken in the indigenous sites with the participation of indigenous people with a target of conserving and revitalising TEK. In practice, TEK conservation has always been tightly associated with, or included in natural resource management and biodiversity conservation (environmental conservation) ${ }^{37}$. As many practitioners and scholars argue, one of the most vital principles of TEK conservation is to conserve it in situ, with local indigenous involvement (e.g. Berkes, 2002, 2007; Brodt, 2001; Gavin et al., 2007; Zent, 1999). This is mainly because TEK is generated from the interactions between humans and their natural environment; extracting TEK from its cultural and physical environment is disconnecting it from its roots (Zent, 1999). In recent years, a community-based environmental conservation approach has been considered appropriate by many researchers and practitioners in comparison with a top-down approach (e.g. Banks et al., 2003; Berkes, 2002, 2007; Gavin et al., 2007; Leach et al., 1999; Tang and Gavin, 2010). In accordance with the resource governance decentralisation process, this approach advocates the devolution of power and responsibilities of environmental conservation to local community level (Gavin et al., 2007). As Gavin et al. (2007) clarify, there is a wide spectrum of the possibilities that exist regarding community involvement in environmental conservation strategies. Below, the category is further divided into three approaches: traditional lifeways programmes, environmental conservation activities, and TEK commoditisation.

\subsubsection{Traditional lifeways programmes are programmes that are indigenous} community-based with an aim to revitalise or promote traditional lifestyles. These programmes often focus on increasing inter-generational TEK transmission, and instilling pride in traditional lifestyles within indigenous youngsters. For instance, in

\footnotetext{
${ }^{37}$ Natural resource and biodiversity conservation is termed as environmental conservation in this thesis to distinguish from TEK conservation.
} 
Pikangikum, Ontario of Canada, the First Nations have carried out a programme named "culture days" for several years. The programme permits indigenous children to be absent from schools in order to participate in hunting and other traditional seasonal livelihood activities. They therefore are able to learn traditional knowledge and practices from their communities (Miller, pers. comm., 2011).

In Winnipeg, Manitoba of Canada, as a community elder described (surv. resp., 2010), "Elder camps provide an opportunity for the youngsters to eat traditional food, and go for plant walks with the elders; Youth challenge organises canoe and camping races for youngsters. They are not allowed to bring any supplies to the camps. Under the supervision of elders, they will learn how to use natural materials to make canoes or to meet camping needs". In the Marshall Islands, an NGO set up a programme to teach traditional navigation to disadvantaged youth, and recently locally-driven initiatives to revive fine handicrafts. For example, annual competition and auction for traditional mats have been held (surv. resp., 2011).

Multiple survey respondents claimed that these actions were achieving a noticeable level of success in terms of maintaining traditional practices and enhancing TEK transmission. As the survey results show (see Chapter 3, section 2.3.1.1 for details), degraded TEK transmission (mainly vertically between generations) is one of the most threatening factors that have caused TEK loss worldwide. A key resolution to deal with this threat is to enhance or re-build the interest and incentives in TEK from within indigenous communities, especially with the youth. The traditional lifeways programmes are targeting on enhancing community members, especially the young members' interest in TEK. The activities provide an opportunity for community members to learn or re-experience their traditional cultural and livelihood practices. For younger generation, knowing is the first step that may lead to the interest in and the pride of their traditional lifestyle, thus increasing the possibility of TEK transmission (Florey, 2009).

2.2.2.2 Environmental conservation activities refer to the indigenous communitybased projects aim to conserve biodiversity and natural resources, in which TEK is protected and TEK holders are involved. Along with the global trend toward the 
decentralisation of resource governance, community level institutions play an increasingly important role in environmental conservation. As Brondizio et al. (2009) state, the decentralisation has emphasised a shift from the conventional top-down resource governance towards co-management, community-based and self-governing resource management. More community-based management regimes allow communities to contribute their traditional knowledge of the local environment to resource management practices (Agrawal and Gibson, 1999). Often, within these practices, communities' traditional rights over the land and resources are recognised and protected (Lemos and Agrawal, 2006).

The survey results and literature review highlighted two types of indigenous environmental conservation activities: community-initiated programmes, and community participation in 'external' conservation programmes.

\section{A. Community-initiated environmental conservation programmes refer to the} environmental conservation programmes initiated and led by indigenous communities within their traditional framework. For example, the Cherokee community of North Carolina USA initiated a heritage seed programme. The programme began in the late 1990s, when the loss of traditional crop varieties became obvious. According to their traditional knowledge, these communities understand that secure food supply depends on maintaining the genetic diversity of crops. The crop variety will enable humans to survive through disease or climate changes. By growing and exchanging the seeds, and creating a seed bank of endangered varieties of crops, the participating community have gained a significant increase of seed varieties, as well as raising public awareness of crop varieties and food security in the Cherokee region (surv. resp., 2011).

The significance of this mechanism is that it achieves the complete control of the projects by indigenous communities. Unlike some externally driven projects, in which community dependence on external management and funds is significant, communityinitiated conservation usually possesses strong incentives and support from inside the community. Therefore these approaches can be more likely to achieve long-term effectiveness and sustainability (Berkes, 2007). 
B. Participating in 'external' environmental conservation programmes refer to the environmental conservation programmes initiated and led by governmental agencies or external organisations with indigenous community's participation. For example, it is a common case that indigenous people are hired as rangers or guards for protected areas. In the survey, this kind of local participation was reported from Australia, Benin, Canada, China, Kenya, Thailand and South Africa (multiple survey responses, 2010-2011). In this way, the local knowledge has been used in conservation management, and the conflicts between conservation and local community have been mediated at a certain extent.

As a researcher (surv. resp., 2010) in Mbirikani, Kajiado of Kenya described, "Our conservation project employs traditional, usually uneducated warriors to monitor carnivores and mitigate conflict in their communities. This not only utilises their TEK and skills but encourages them to pass on the importance of these to the upcoming warrior age set. In order to monitor the carnivores, they use their tracking skills, knowledge of the bush (including types of habitats etc) and animal behaviour and also herding knowledge to prevent livestock wandering into areas of depredation risk".

Though, in comparison with community-initiated conservation activities, participating in externally-driven conservation might not achieve the same level of indigenous community autonomy and self-determination, this approach at least may facilitate the recognition of the values of TEK and allows community involvement. This is a positive move towards a more collaborative relationship between community and external organisation/governmental agencies, and forms an important progress in resource governance decentralisation.

2.2.2.3 TEK commoditisation refers to the transformation of aspects of TEK, which are not normally regarded as having monetary value, into commodities. Through commoditisation, TEK may gain appreciation from both insiders and outsiders of an indigenous community, as well as gain financial benefits for the community. Within this process, TEK will have a better chance to be transmitted.

A. Ethno-tourism or eco-cultural tourism refers to tourism oriented towards indigenous culture, local landscapes/seascapes, or local species. Ethno-tourists are 
generally outsiders who appreciate the uniqueness of a given indigenous group's culture and their location (Butler and Hinch, 2007). Within ethno-tourism activities, the local artifact and traditional cultural expressions are often presented as a showcase to the tourists (Timothy, 2011). For instance, in Shuar territory, Morona-Santiago Province of Ecuador, an NGO helped local communities to initiate ethno-tourism enterprises in the area. They are showcasing traditional song, dance, costume, and selling crafts to tourists (surv. resp., 2010). In Kibale National Park, Kabarole District of Uganda, there are many local community organisations that practice traditional dances and songs with the intention of attracting tourists. However, so far, there is not much market for them (surv. resp., 2010).

A researcher (surv. resp., 2010) who studied in thirteen villages in Panama Province and Darien province of Panama illustrated, "Ethno-tourism has recently become an important source of income for many communities, and in these communities the performance of traditional dances and music, many of which represent local wildlife or seasonal events, have become important sources of cash".

As many scholars and survey respondents address, ethno-tourism brings opportunities for community development, in which community economic and institutional capacity can be strengthened, and community's market opportunities are enhanced (McIntosh and Ryan, 2007; McIntyre and Gosford, 2011). The function of poverty reduction by operating ethno-tourism has also been highlighted by some scholars (e.g. Butler and Hinch, 2007; Goodwin, 2007; Scheyvens and Momsen, 2008). However, there are significant controversies surrounding ethno-tourism, including three key dimensions: first, outside tourists can bring the indigenous community into contact with diseases they do not have immunities against (Butler and Hinch, 2007); second, cultures can be degraded or destroyed upon contact with non-indigenous cultures (Timothy, 2011; Timothy and Boyd, 2003; Welch, pers. comm., 2010); and third, ethno-tourism can be associated with significant environmental impacts (Hinch, 1998). Thus, as the United Nations Environment Programme (UNEP) and World Tourism Organisation (WTO) (2005) suggest, cultural heritage oriented tourism should be designed with sustainability, the traditional culture, indigenous social characteristics and local environment should not be compromised at any extent. 
B. Indigenous products trading refers to small scale trading activities of indigenous products, such as handicrafts, bio-resource products, traditional tools, etc. The production unit is usually small scale, which is single family or neighbourhood based, and only sometimes community-wide. The products are usually traded locally through local markets or sometimes to wider areas even internationally through trade-aiding organisations. Indigenous products trading have been claimed from multiple countries, such as Australia, China, Ecuador, Kenya, Mexico, Panama and Uganda. For example, in Swaziland, an indigenous trading organisation - Swazi Indigenous Products (SIP) was established in 2004 with the aim of creating new income generating activities for rural Swazi women. The organisation is community-initiated and community-owned, and the profit is destined for the participating rural women themselves (SIP, 2012). One of their main products is Marula oil, which is produced from wild harvested fruits. The oil both sold as a raw material to the global cosmetics market, as well as being formulated into a range of locally made finished cosmetics products that are available both domestically and internationally. As the organisation summarises, "sharing the benefits that result from trade in local biodiversity in a way that helps support local, sustainable development and traditional techniques and practices is at the heart of SIP's work" (UEBT, 2012; see more information on http://www.swazisecrets.com).

In Tenejapa, Chiapas of Mexico, as a community member observed (surv. resp., 2010), "There is revival of traditional back strap loom weaving. The technique had disappeared for several years, but now women are revising it. They are using natural dyes for weaving. This revival has been motivated by the development of new coop marketing outlets". In Panama and Darien provinces of Panama, a researcher reported (surv. resp., 2010), "Traditional tools and crafts have also become important sources of income, and even in remote communities some people now dedicate the majority of their time to the production of baskets and carvings, which they sell for cash to intermediaries".

As the above examples demonstrate, the trading of indigenous products can generate income for participating community members; it therefore promotes the values of making these traditional products, in which traditional knowledge and skills are maintained or revitalised (Akinnifesi et al., 2008; Lasimbang, 2008; LeClair, 2008). Meanwhile, these community-initiated trading activities commonly apply the traditional, 
equitable benefit-sharing mechanism, which strengthens their indigenous institutional principles (Lasimbang, 2008; UEBT, 2012).

\subsubsection{Action Three - Education and awareness}

Education and awareness refers to the actions that conserve and promote TEK through educational systems and public awareness raising. Education has been claimed to be an important tool for TEK conservation by a number of scholars and survey respondents. Based on the fact that the implementation of formal education has significantly contributed to TEK degradation (e.g. Benz et al., 2000; Ellen, 2007; Godoy et al., 2008; Lizarralde, 2001; Quinlan and Quinlan, 2007; Reyes-Garcia et al., 2005; Robert, 2004; Zent, 2001), many scholars advocate the development of indigenous education (e.g. Heckler, 2007; Kanu, 2006; Kincheloe, 2006; May, 1999; Niroa, 2004; Robert and Angela, 2004). The survey and literature review identified three approaches for implementing indigenous education: including TEK in formal education systems, customary education systems, and indigenous media and awareness mechanisms.

2.2.3.1 Inclusion of TEK in formal education refers to advancing TEK transmission through incorporating TEK and indigenous languages in the formal education system. The idea of including indigenous language and culture into public schools and universities has received significant support in recent years. Such integration has been carried out to varying degrees in many countries, such as Australia, Benin, Canada, China, Italy, New Zealand, Peru, South Africa, Tanzania, Vanuatu, Vietnam, Uganda and USA (surv. resp., 2010-2011). The TEK teachers may be indigenous knowledge-holders, or trained teachers within the formal education system.

For example, in Cherokee, North Carolina of USA, traditional arts have been introduced into public schools. Indigenous crafts-people teach traditional skills in schools, such as river cane basket weaving. A native language revitalisation programme is also carried out in schools (surv. resp., 2010). In Pikangikum, Ontario of Canada, the government supports the formation of school programmes for native language retention. Native speakers, often the elders, are invited or hired as instructors of culture and language classes in public schools (surv. resp., 2010). Also in Canada, based on indigenous 
knowledge, the guidebook of local plants and animals, and custom uses of these resources has been developed, and is widely used in the school curriculum in Winnipeg of Manitoba (surv. resp., 2010). In Loreto region of Peru, a survey respondent observed (2010), "there is promotion of bilingual education, which incorporates native language in school curriculum; old persons tell stories and customs in cultural classes".

These examples illustrate that the inclusion of TEK and indigenous languages in formal education has been accomplished to a certain degree in some locations. However, the extent and nature of these programmes differ between regions, and there are no standard procedures to direct its implementation. In most countries, formal education is still dominated by Westernised ideology and subjects, and TEK is merely included in a supplementary fashion. By using multiple examples from more than 20 island nations of Oceania, Thaman (2002) points out that indigenous culture should be the foundation for education rather than a variable within it. This is because learning occurs within a particular cultural context. In agreeing with Thaman, Kanu (2003:69) discusses curriculum reform in Canada in the terms of "curriculum as cultural practice". He notes that, "making cultural equity very important in contemporary understanding of educational reform, especially as reform relates to social inclusion and to the relation of power". Thaman and Kanu both emphasise the importance of treating indigenous culture as a fundamental concern in formal education design. A deeper recognition and a wider application of the integration of indigenous context in formal education are highly desirable.

2.2.3.2 Customary education refers to the educational system initiated and managed by indigenous communities with the aim of transmitting TEK and promoting traditional knowledge, culture and lifestyle. This system may include kindergarten, primary, secondary and tertiary levels. Customary education, or in another term, indigenous community-based education as a parallel system to the formal education has been promoted by multiple scholars (e.g. Carson, 1999; Fettes, 1999; May, 1999; McCarty and Lucille, 1999). As May (1999:1) points out, "indigenous community-based education has been developed in recent years as a response to the long historical colonisation, subjugation and marginalisation of indigenous people". It is predicated on, and framed within the wider indigenous self-determination processes. 
For example, in Whakatane, North Island of New Zealand, a Māori indigenous university - Te Whare Wānanga o Awanuiārangi was established in 1992 and officially became a Wānanga (university) in 1997 (http://www.wananga.ac.nz). The vision of the university is to empower all Māori to claim and develop their cultural heritage and to broaden and enhance their knowledge base so as to be able to face with confidence and dignity the challenges of the future. The university provides a wide range of majors and courses with a significant emphasis on Maori history and traditions, Maori identities and Iwi (Maori tribes) development. The graduates from the university have high recognition from the society and are very competitive to graduates from formal universities (pers. comm., 2010).

In Malekula Island, Vanuatu, the Vanuatu Cultural Centre actively supports local communities to develop rural customary schools. The aim of this project is to conserve the traditional practices within communities. The schools invite community elders to teach indigenous language, traditional practices and skills (McCarter, pers. comm., 2011). The Cultural Centre also established an urban customary school in 2008 . The aim of the project is to empower young people in Vanuatu to take control of their lives, advocate their interests more effectively, and have their needs addressed by their communities and policy makers. This project provides an opportunity for young people to participate and learn their customs and culture, encourages and promotes the use of vernacular language, and encourages young people to develop their own skills using valuable resources to generate an income (see more information on http://www.vanuatuculture.org/site-bm2/ypp/vanuatu-young-peoples-pro.shtml).

These examples reflect that customary education allows community members to become self-oriented participants in the creation of the learning environment; it also allows them to become meaningfully involved in shaping their future through their own educational system (Carson, 1999; May, 1999). As Carson (1999) points out, customary education "meets the needs and interests of diverse communities who want to free themselves from pressure to conform the unwanted, dominant, cultural structures". However, we have to notice that, though the success of customary education was claimed from above examples; the application of customary education is still far from 
being popular and common at a global scale (Lasimbang, 2005). Self-determination in education is one of the basic human rights of indigenous peoples; and states should develop strong efforts to and provide support for the educational innovations sought by indigenous communities (Kaunga, 2005).

2.2.3.3 Indigenous media and informal learning refers to raising awareness of TEK and its values amongst indigenous communities and general public through media communication tools and informal learning processes. While including TEK in the formal education and custom education targets on indigenous students, this action targets on wider public.

A. Indigenous media refers to raising awareness and recognition of TEK and other indigenous traditions by applying communication tools, such as newsletters, radio stations, and websites. As Ginsburg (1993) addresses, indigenous media provides a vehicle for indigenous cultural and political communication by indigenous peoples to themselves, and to the non-indigenous societies. From the perspective of many indigenous producers, indigenous media are a powerful means of collective selfexpression that have a culturally revitalising effect (Ginsburg, 1991; Turner, 1990). Politically, indigenous media are also considered as a major means to challenge the concentration of media power (Couldry and Curran, 2003). This is based on the fact that media is the holistic result of countless battles over "who has the power to represent the reality of others" (Couldry and Curran, 2003). For a long period, indigenous people's voices have been undermined by the mainstream media. Only along with the development of indigenous rights movements and of alternative media in recent decades, indigenous media have had a chance to develop.

For example, in Ngati Raukawa ki te tonga, Te Ati Awa ki Whakarongotai and Ngati Toarangatira tribal areas, Lower North Island of New Zealand, community newsletters (Kai Korero) have been created for the communities to tell their stories of current lifes and traditional practices. The newsletters are edited and printed in the Māori language, and exchanged among communities (surv. resp., 2010). In Pino Gordo, Chihuahua of Mexico, there is a local radio show in Raramuri which communicates information of present lifes, traditional livelihood and cultural practices in native language. Several 
local community controlled radio programmes also exist in Pikangikum, Ontario of Canada.

Indigenous websites have also been developed rapidly over the last decade. For example, Digital Indigenous (http://www.digitalindigenous.com/) is a website created and managed by Māori people. The main mission of the website and the company behind is "providing training, strategic advice, project management, management and consultancy services within the health and other sectors in Aotearoa/New Zealand, and is working closely with organisations that are interested in advancing Māori health and wellbeing including: iwi; government agencies; district health boards; NGOs; Māori health providers; community organisations and private entities".

\section{B. Informal learning refers to raising awareness of TEK and promoting TEK} transmission through non-formal learning means, such as botanical gardens, indigenous museums and exhibitions, and workshops. For example, in Infierno and Sonene of Peru, ethno-botanical gardens have been running for more than 15 years. The Ese'eja botanical garden was established by the community in early 1990s. It contains over 200 species of native plants, and has rapidly become an important learning centre for local and international visitors (surv. resp., 2010). Similarly in Chile, the Omora ethnobotanical park functions as: 1) an outdoor classroom that open to students and public who are interested in nature, its landscapes and cultures; 2) a natural laboratory to study the ecology of the southernmost forests on the planet; and 3) a public space of promoting harmonious living between human beings and other biological species (see more information at http://www.omora.org/english/welcome.htm).

In Chitwan National Park of Nepal, a tharu (literately means traditional) museum was established in 1998, and an indigenous medicinal herbs nursery was established in 1999. These projects provide physical space for public, especially the youth to learn indigenous traditions, culture and knowledge of cultural important plants and animals (surv. resp., 2010) (see more information on http://chitwannationalpark.net).

A number of workshops regarding traditional knowledge and practices have been claimed from multiple countries. For instance, in Chitwan National Park of Nepal, 
several Indigenous healer workshops have been undertaken; in New Zealand, community hui and wānanga (learning workshops) have been held; and in Infierno and Sonene of Peru, some workshops for traditional medicinal plants knowledge exchange are undertaken (survey responses, 2010).

From above example we can see that informal learning can take place in various places, which are usually non-formal-educational settings, through daily interactions and shared relationships among members of a community or society (Cofer, 2000).

Distinguishing from formal learning, informal learning is often generated by local people themselves. The learning objectives and goals are designed by the "learners" rather than "educators". This setting is more likely to lead to wider community involvement and activism (McGivney, 1999). The significance of informal learning has been discussed in the literature, though there is a lack of a specific focus on the indigenous perspective. According to Eraut (2004), informal learning provides greater flexibility or freedom to learners. It recognises the social significance of learning from other peoples, and promotes cultural identity of a given community. As Wenger (1999) points out, social participation within the community is the key to informal learning. It is embedded in the practices and relationships of the given community and helps to create identity and meaning. Informal learning can both complement and substitute formal learning mechanisms.

\subsubsection{Action Four - Policy and legislative support}

The category of policy and legislative support refers to the actions of developing formal legislation and policies, and influencing and promoting their implementation in regards to indigenous rights protection and TEK conservation. If we draw our attention back to Chapter Three, policy and legislation is identified as a major underlying threat, which can cause multiple direct threats to TEK (loss of pathways of TEK transmission, loss of traditional livelihood practices, loss of traditional rights and loss of traditional institutions). While TEK-unfriendly policy and legislation may effectively and 'efficiently' cause TEK loss, appropriate policy and legislation may also effectively and efficiently conserve TEK and indigenous rights. Below, I will discuss how policy and legislation can function as a TEK conservation mechanism at multiple levels, from international to 
national and regional.

\section{International level}

There is a great amount of literature reviews the values and implementations of international policy and legislation regarding indigenous rights protection. It would be impossible to cover all this literature under this section. Below, I provide a brief review of the development of relevant international laws and policies, with examples from the survey and the literature (in order to avoid repetition, I mainly list the policy and legislation has not been included in section 1.2 of this chapter).

Since the $18^{\text {th }}$ century, most indigenous groups have petitioned for the recognition of their cultural and sovereignty rights since the creation of coloniser or central state exclusive authority (Dickason, 1989). However, this principle was too often denied by the authorities in colonised lands (Dickason, 1989). Frustrated by failures to secure sufficient rights in their own homelands, many indigenous groups lobbied for international recognition and support (Mauro and Hardison, 2000). For example, the United Nations (UN) has continuously received indigenous claims for their indigenous rights since it was established in 1945. As a result, a series of UN declarations and policy concerning indigenous rights have been developed over the last six decades.

The UN Charter (1948, can be accessed via http://www.un.org/en/documents/charter/ index.shtml) recognises the free-pursuit and self-determination of non-self-governing territories, and this led to a period of decolonisation. However, in this Charter, the UN interpreted the obligations to extend only to the external colonies, and not to indigenous peoples living in internal enclaves (Lepage, 1994). The rights embedded in the UN Charter were based on universal human rights, and it was thought that no special grouprelated rights were needed to protect indigenous peoples (Mauro and Hardison, 2000).

The UN in the late 1950s recognised that the universal human rights provisions were not enough to protect ethnic minorities and indigenous peoples from persecution, assimilation and genocide. The UN International Labour Organization (ILO) in 1957 adopted ILO Convention 107 (can be accessed via http://www.ilo.org/global/about-theilo/lang-en/index.htm), which recognises indigenous rights to customary law, social 
organisation, land tenure, collective land ownership, and customary practices. However, these were conceived as individual rather than sovereign rights, and were promoted primarily to integrate indigenous peoples into the labour pools of the modern nationstate (Lepage, 1994). The Convention did not receive wide support, and has been ratified by only 27 countries.

There are also a number of other UN declarations and conventions concerning indigenous cultural and language rights. One of the most influential pieces is the Declaration and International Convention on the Elimination of All Form of Racial Discrimination (1969, can be accessed via http://www.unhcr.org/refworld/ docid/3ae6b3940.html) that officially addresses the problem of discrimination against indigenous populations (Lepage, 1994). The report concludes that, "states should respect traditional laws and customs; indigenous peoples should have control over their own lands and resources, with the right to communal land ownership and to manage land according to their own traditions; and such ownership and rights should be protected by national and international laws".

Following the recommendation of the report, the UN Commission on Human Rights established the Working Group on Indigenous Populations (WGIP) in 1982. The WGIP reviews the evolution of standards concerning the rights of indigenous peoples, provides a forum where they can express themselves, and promotes the protection of their rights. The United Nations Declaration on the Rights of Indigenous People (first drafted in 1988, recently amended in 2008, can be accessed via http://www.un.org/esa/socdev/ unpfii/documents/DRIPS en.pdf) stipulates rights to self-determination, collective rights, cultural and intellectual property rights, and obligates states to observe treaties (Anaya, 1996)(see more details in section 1.2). The Declaration recognises the urgent need to respect and promote the inherent rights of indigenous peoples which derive from their political, economic and social structures and from their cultures, spiritual traditions, histories and philosophies, especially their rights to their lands, territories and resources. It also recognises that the respect for indigenous knowledge, cultures and traditional practices contributes to sustainable and equitable development and proper management of the environment (Kastrup, 1997). Its 46 articles fully sets out the individual and collective rights of indigenous peoples, and 
their rights to culture, identity, language, employment, health, education and other issues (Dorough, 2009).

The WGIP has also produced a global study of treaties between states and indigenous peoples, and is currently investigating options for protecting their cultural and intellectual property (Daes, 1999). These initiatives are all important international policy instruments that construct indigenous rights and link rights to culture, language, religion, land, and resources, including biodiversity (Mauro and Hardison, 2000).

\section{National and regional levels}

In Canada, the first policy on Aboriginal claims was established in 1973, along with processes and funding for resolving these claims through negotiation. These are optional processes that provide Aboriginal groups with an alternative to going to court to resolve their claims. Section 35 of the Constitution Act 1982 explicitly recognised and preserved aboriginal rights. In 1982, the first decision of the Supreme Court of Canada after the Constitution Act 1982 declared that the aboriginal title was sui generis and that the federal government has a fiduciary duty to preserve it. A significant number of aboriginal land claims have been achieved since then (see more information on http://www.aboriginalcanada.gc.ca/acp/site.nsf/eng/ao20009.html). As a research in southwest Yukon Territory and Nunavut Territory of Canada observed, the settlement of Aboriginal land claims has given substantive weight and power to collection and use of TEK in co-management decision processes (Clark, pers. comm., 2010).

In the Unites States of America, the unique status of indigenous peoples was recognised during the treaty-making years of 1778-1868. The powers granted in the U.S. Constitution to make treaties were recognised as powers to conduct foreign relations (Prucha, 1994). Treaties often referred to tribes as "nations," established peace between the Indian nations and the United States, created boundaries for tribal lands, and granted a degree of autonomy within those lands. Prior residency and distinct languages, cultures, laws, and governance were considered to grant tribes sovereign status (Prucha, 1994). 
In Australia, a number of government programmes and policy (both at state and federal levels) support indigenous traditional practices and traditional knowledge, mainly through National Parks and Wildlife Service. As a researcher (pers. comm., 2010) described, "Aboriginal rangers are looking after TEK sites, and are documenting TEK and creating TEK databases. They are also in charge of organising sporadic visits to the sites, and have the right to limit tourist promotion if it harms their TEK".

In Huamachuco and surrounding villages, La Libertad of Peru, as a researcher (surv. resp., 2010) reported, "The top herbalists from the various towns have been appointed as public health officials by the ministry of health, and medicinal plant use seems to be generally accepted and encouraged by the local hospital staff and management".

Though, the existing law and policy has not always resulted in real action. To date, the governments of USA, Canada, Australia and Peru have continuously been criticised for lacking sincere motion for protecting indigenous rights (e.g. Hodgson, 2002; Marcus, 2002; Mauro and Hardison, 2000). The above examples illustrate that a national policy and legislative framework for protecting indigenous rights should be one essential conservation mechanism to TEK, but how to transform these policy and legislation into real-world actions at ground levels is more important. Meanwhile, it is undeniable that international laws and policy are one of the most influential driving forces that lead to corresponding national and regional legislation and policy.

\section{Indigenous intellectual property rights}

Despite the debate regarding the effectiveness and suitability of intellectual property rights (IPRs) for TEK conservation, IPRs have been widely discussed and recommended in the literature. The intellectual property rights of TEK are a major concern of both indigenous peoples and TEK researchers, and the best way to protect these rights has been a popular topic in the field.

A significant number of scholars suggest the use of the existing Intellectual Property Rights system, such as patents, trademark and copyright, to protect TEK (e.g. Mathur, 2003; Mugabe, 2000). The World Intellectual Property Organisation (WIPO) has produced numerous comprehensive reviews of existing intellectual property tools for 
protecting TEK and providing benefits sharing. The Intergovernmental Committee on Intellectual Property and Genetic Resources, Traditional Knowledge and Folklore (the IGC) of WIPO met for the first time in 2001 to discuss the draft provisions for the enhanced protection of traditional knowledge and traditional cultural expressions against misappropriation and misuse (WIPO, 2011). WIPO's work in these areas involves close cooperation with other international organisations and NGOs, as well as the organisation of a wide range of capacity building activities. Capacity-building resources include practical guidelines for indigenous and local communities on developing intellectual property protocols, and information technology tools for managing intellectual property issues.

In spite of the large quantity of literature that discusses the potential values of using IPRs in TEK conservation, only two survey respondents claimed the actual use of the intellectual property law in their cases. Based on his research with San people in South Africa, Stephenson (2010) provides a valuable example of using the IPR system to achieve benefit-sharing for indigenous groups:

"In March 24 [2010], elected representatives of the San peoples in South Africa, known as the South African San Council, and the Council for Scientific and Industrial Research (CSIR), an arm of the South African government, jointly announced that they had reached an agreement to share the benefits projected to result from the development of a potential blockbuster diet pill [derived from the Hoodia plant, originally found in South Africa, mainly marketed in the USA]. This agreement was the dramatic culmination of a highly publicized confrontation between representatives of the San people and CSIR and a series of negotiations that had begun less than two years earlier. The agreement is a landmark for all indigenous peoples, not only because it provides the San with significant monetary benefits for the commercial exploitation of the intellectual property that is inherent in their traditional knowledge, but also because it serves as a testament to the enduring value of the San cultural heritage."

He also pointed out, "In this case, the IPRs mechanism used is not really the patent, but traditional knowledge compensation. IPRs are not ideal for the situation, but they a tool that can be used". For future development of IPRs protection of TEK, he recommended, 
"Sui generis ${ }^{38}$ would better fit in TEK protection. Lawyers and scientists need to be involved in the process. The government support is also necessary" (Stephenson, pers. comm., 2010).

Disagreeing with the use of existing IPRs, a great number of indigenous community members, practitioners and scholars argue that the existing intellectual property laws offer limited scope for the recognition of indigenous people's rights (e.g. AHRC, 2008; Mead, 2005; Swiderska, 2001; UNPFII, 2007). This is mainly because IPRs are designed to protect commercial inventions, and mostly grant individuals exclusive rights; whereas TEK of communities is first and foremost for subsistence and it is largely held collectively as an ancestral heritage (e.g. Song, 2010; Swiderska, 2001, 2004). According to UDPFII (United Nations Permanent Forum on Indigenous Issues) (2007:97), "Collective intellectual property aspects of indigenous traditional knowledge is not simply a different type of intellectual property; it is a completely different entity". The IGC of WIPO states that the recognition and protection of indigenous traditional knowledge has largely taken place within the parameters of intellectual property law, yet recognises that this has been limited due to the Western constructs of intellectual property laws which fail to be able to accommodate the vastly different requirements for the protection of indigenous traditional knowledge, such as the communal transgenerational concepts of ownership, versus a focus on creativity and individualism (UNPFII, 2007).

Some scholars suggest an alternative intellectual property strategy - Sui generis system (literally means "in one's own kind") for protecting the knowledge, innovation and practices of indigenous and local communities (Dutfield, forthcoming; Mead, 2005; Swiderska, 2009). It may include database rights, plant breeders' rights and other indigenous intellectual property rights (Dutfield, forthcoming). They argue that, Sui generis should not be consistent with existing IPRs models. Instead, it should be designed according to the distinct attributes of traditional knowledge and innovation processes, such as holistic character, free sharing and exchange of resources, collective custodianship and spiritual beliefs (IIED, 2006; Swiderska, 2006). However, the practical use of Sui generis systems has not been reported in either the literature or the survey.

${ }^{38}$ Sui generis is an intellectual property rights system specifically designed for TEK and TEK holders. 
To date, indigenous peoples are having an evolving status in international law and policy. They are participating in defining their rights, in developing international laws and policy, and impacting national laws and policy (Mauro and Hardison, 2000). There will be ongoing tension between indigenous peoples and governments, as they will continuously pressure governments at multiple levels to recognise their rights of selfdetermination and sovereignty, while governments have often sought to limit these rights according to national interest (Mauro and Hardison, 2000).

\subsubsection{Action Five - Research and documentation}

This category refers to studying and recording of TEK by TEK holders, outside institutions, or their joint efforts. According to the survey and literature review results, research and documentation is the most frequently used mechanism in TEK conservation so far. The academic and public attention about indigenous people and their TEK has been brought by scientific research of TEK in the anthropology field back to the 1950s. This kind of research has been widely carried out in Africa, Latin America, North America, Oceania and Asia since then.

In the literature, TEK research and documentation is sometimes referred to as ex situ conservation of TEK. The logic of ex situ conservation is that by recording knowledge, TEK can be preserved, and indigenous communities will gain the recognition of its contribution to the global knowledge system (Agrawal, 2002; Warren et al., 1996). As Agrawal (2002) argues, databases on indigenous knowledge systematically document specific elements of knowledge for later analysis. He also points out that, the objective of the databases is twofold: 1) to protect indigenous knowledge in the face of myriad pressures that are undermining the conditions under which indigenous peoples and knowledge thrive; and 2) to collect and analyse the available information, and identity specific features that can be generalised and applied more widely in service of more effective development and environmental conservation. Since the 1950s, some countries have established TEK databases, such as Australia, India, New Zealand and Peru. 
However, a number of researchers criticise ex situ conservation as it extracts TEK from its local context, and does not necessarily provide benefit sharing with TEK holders (e.g. Brodt, 2001; Zent, 1999). They also argue that TEK is a dynamic system which changes over time, but ex situ conservation does not provide protection for the TEK change. Participatory TEK research and documentation then has become popular in recent years, alongside with the popularity of community-based conservation approach in resource management and conservation regimes (Wilmsen et al., 2008). Commentators argue that in situ conservation is based within the local context and works with indigenous communities, and addresses TEK holders' needs and preferences (Zent, 1999). The results shown below embrace both ex situ and in situ research and documentation.

2.2.5.1 TEK research and documentation refers to the studies and records of TEK carried out by TEK holders, outside institutions, or their joint efforts. The form of documentation may include scientific publications, community archive books, digital/analogy media and other forms. According to the survey results, the research and documentation of TEK has focused on the knowledge domain (46\% of the responses which claimed TEK conservation actions have been undertaken), especially in the field of ethnobiology (24\%). Twenty-five per cent of the research and documentation focus on traditional practices and management regime; $16 \%$ on institutions; and only $2 \%$ on worldviews and beliefs. There are also $11 \%$ of the research and documentation that focus on other aspects of TEK, such as indigenous language, local history and traditional material culture (see detailed information in Table 4.3 below). 
Table 4.3 What has been studied and documented regarding TEK

- Result from the survey $(n=74)$

\begin{tabular}{|c|c|}
\hline $\begin{array}{c}\text { Domain of TEK } \\
\text { (\% of the responses under this category) }\end{array}$ & Aspect of Study \\
\hline \multirow[t]{3}{*}{ Knowledge (46\%) } & $\begin{array}{l}\text { 1. Names and other knowledge of species (including } \\
\text { plants and animals) (e.g. local plant inventory; local } \\
\text { bird inventory) }\end{array}$ \\
\hline & $\begin{array}{l}\text { 2. Names and associated stories of places (e.g. } \\
\text { inventory of local mountains and rivers) }\end{array}$ \\
\hline & 3. Landscape/seascape \\
\hline \multirow[t]{3}{*}{$\begin{array}{l}\text { Resource use and management } \\
\text { practices }(25 \%)\end{array}$} & $\begin{array}{l}\text { 1. Land use and livelihood practices } \\
\text { (e.g. traditional harvest species; traditional food source } \\
\text { and food preparation) }\end{array}$ \\
\hline & $\begin{array}{l}\text { 2. Resource use and management techniques (e.g. } \\
\text { organic agriculture) }\end{array}$ \\
\hline & $\begin{array}{l}\text { 3. Other traditional practices and techniques (e.g. } \\
\text { healing practices) }\end{array}$ \\
\hline \multirow[t]{2}{*}{ Institutions (16\%) } & $\begin{array}{l}\text { 1. Social organisations (e.g. traditional leadership, } \\
\text { election process) }\end{array}$ \\
\hline & $\begin{array}{l}\text { 2. Customary rules of resource use and management } \\
\text { (e.g. traditional fishing quota) }\end{array}$ \\
\hline \multirow[t]{2}{*}{ Worldviews and beliefs (2\%) } & 1. Spiritual beliefs (e.g. resource guardianship) \\
\hline & 2. Ceremony \& rituals (e.g. adulthood ceremony) \\
\hline \multirow[t]{3}{*}{ Other $(11 \%)$} & $\begin{array}{l}\text { 1. Local history (e.g. transformation of environment } \\
\text { and knowledge since European settlement in Australia; } \\
\text { land occupation in USA) }\end{array}$ \\
\hline & 2. Traditional languages \\
\hline & $\begin{array}{l}\text { 3. Traditional material culture (e.g. costumes; pottery; } \\
\text { weaving ) }\end{array}$ \\
\hline
\end{tabular}


The above table shows that study and documentation have covered all the domains and dimensions of TEK according to Berkes's (2008) definition of TEK ${ }^{39}$, though with uneven weight. These research and documentations may enhance the chance of TEK to be conserved and transmitted.

2.2.5.2 TEK databases refer to the organised collection of data regarding indigenous knowledge, traditional practices and the cases of TEK conservation with an aim of maintaining TEK. The early development of TEK databases was carried out mostly by developed countries or international organisations, and only recently communityinitiated and community-managed databases have emerged (Myer, 2000). In the last 20 to 30 years, to follow a truly participatory research principle, the control of database access has become a key concern. For instance, as DEWHA ${ }^{40}$ (2009) emphasises, indigenous communities can record and manage their own information and should be a critical feature of indigenous database systems. Below, I give a few examples of databases created by different organisations and at international and national levels.

\section{International level databases}

The UNESCO's Management of Social Transformation Programme (MOST) has established the Database of the Best Practices on Indigenous Knowledge in 1999 in cooperation with the Netherlands Organization for International Cooperation in Higher Education and Indigenous Knowledge (NUFFIC/IK-Unit) (http://www.unesco.org/ most/bpindi.htm). The database contains examples of successful projects which illustrate the use of local and indigenous knowledge in the development of cost-effective and sustainable survival strategies, covering Africa, Asia-Pacific, Europe, North America, Latin America and the Caribbean. This database also includes a geographical and thematic index, and an index of institutions acting as indigenous knowledge resource centres. As the database highlights, "it is very important that information about this kind of projects is made available world-wide so that other people can learn from the experiences. The database of best practices will play an important role in building a bridge between empirical solutions, research and policy" (UNESCO/MOST, 2012).

\footnotetext{
39 TEK includes four domains - knowledge, practices, institutions and worldview and beliefs. 40 Department of the Environment, Water, Heritage and the Arts of Australian Government
} 


\section{National level databases}

As a product of the Indigenous Information Management workshop, the Australian Indigenous Knowledge Management Database (AIKMD) was created in 2008 (http://www.environment.gov.au/indigenous/publications/pubs/knowledgemanagement-guide.pdf). The database uses GIS and archival systems to accommodate and present the data regarding indigenous knowledge, traditional practices and their use in resource management and conservation nationwide. The aim of the database is to provide an opportunity for information sharing between indigenous communities, groups and other interest-organisations. As AIKMD (2009) addresses, one of the key features of the database is that communities can record and manage their own information; within the processes of creating and managing the database, as many community members highlight, it provides a chance for young community members to learn and appreciate TEK.

In some senses, the research and documentation of TEK have functioned as something of an 'ark' for bodies of TEK worldwide, and some knowledge has been recorded before it disappears. However, as some scholars and survey respondents point out, research and documentation are passive conservation mechanisms compared with the active use of TEK. One researcher (pers. comm., 2010) stated that, "the documentation does not improve TEK transmission; rather, it only records TEK in books". In a combination with other more active conservation mechanisms, research and documentation functions as a fundamental part of the whole TEK conservation system.

\subsection{TEK threats and conservation actions defined by geo-regions}

In this section, I will combine the results from this chapter and Chapter Three to compare the top direct threats and top conservation actions by geo-regions (based on the frequency listed by survey responses). This analysis aims to explore if there is any association between TEK direct threats and current conservation actions (Table 4.4). 
Table 4.4 Commonly Listed TEK Threats and Conservation Options by Geo-regions

\begin{tabular}{|c|c|c|}
\hline $\begin{array}{c}\text { Geo- } \\
\text { regions }\end{array}$ & Top threats & Top actions \\
\hline Africa & $\begin{array}{l}\text { * Loss of pathways of TEK transmission } \\
* \text { Change of traditional livelihood practices } \\
\text { * Change of environment and natural } \\
\text { resources }\end{array}$ & $\begin{array}{l}\text { * Research and documentation } \\
\text { * Community-based TEK conservation } \\
\text { * Education and awareness }\end{array}$ \\
\hline Asia & $\begin{array}{l}\text { * Change of traditional livelihoods } \\
\text { * Loss of pathways of TEK transmission } \\
\text { * Change of traditional religion and beliefs } \\
\text { * Change of environment and natural } \\
\text { resources }\end{array}$ & $\begin{array}{l}\text { * Research and documentation } \\
\text { * Community-based TEK conservation } \\
\text { * Education and awareness }\end{array}$ \\
\hline $\begin{array}{l}\text { North } \\
\text { America }\end{array}$ & $\begin{array}{l}\text { * Loss of pathways of TEK transmission } \\
{ }^{*} \text { Change of traditional livelihood practices }\end{array}$ & $\begin{array}{l}\text { * Research and documentation } \\
\text { * Community-based TEK conservation } \\
\text { * Education and awareness } \\
\text { * Capacity building } \\
\text { * Policy and legislative support }\end{array}$ \\
\hline $\begin{array}{l}\text { South } \\
\text { America }\end{array}$ & $\begin{array}{l}* \text { Change of traditional livelihood practices } \\
* \text { Change of traditional religion and beliefs }\end{array}$ & $\begin{array}{l}\text { * Research and documentation } \\
\text { * Community-based TEK conservation } \\
\text { * Capacity building } \\
\text { * Policy and legislative support }\end{array}$ \\
\hline Europe & * Change of traditional livelihood practices & $\begin{array}{l}\text { * Research and documentation } \\
\text { * Education and awareness }\end{array}$ \\
\hline Oceania & $\begin{array}{l}\text { * Loss of pathways of TEK transmission } \\
{ }^{*} \text { Change of traditional livelihood practices }\end{array}$ & $\begin{array}{l}\text { * Research and documentation } \\
\text { * Community-based TEK conservation } \\
\text { * Education and awareness } \\
\text { * Policy and legislative support } \\
\text { * Capacity building }\end{array}$ \\
\hline
\end{tabular}

(Note 1: The order of threats and action indicates the frequency listed by the survey respondents; 2 : the cut off rate was $\geqslant 30 \%$ of the frequency to be listed for both threat and action)

By linking the frequency of TEK threats, conservation actions and their geographical distribution addressed by the survey responses, this research notes that: 1) the application of research and documentation $(n=74)$ is the most commonly used option in all geo-regions. It has been carried out mostly in Africa, North America and Asia; 2) the second most frequently used option is community-based TEK conservation activities $(\mathrm{n}=48)$, which has been claimed most frequently in Africa and North America; 
3) education and awareness $(n=30)$ has also been reported as most frequently used in Africa and North America; 4) capacity building (22) has been carried out mostly in North America and South America; and 5) policy and legislative support ( $n=17)$ has been used more often in North America, South America and Oceania.

In respect of associations between TEK direct threats and conservation actions, however, Table 4.4 suggests that there are no clear linkages between different TEK threats and the conservation actions undertaken. In the next section (3.1.1), I will provide detailed discussion and suggestion of corresponding TEK conservation actions for each direct threat.

\subsection{The assessment of TEK conservation actions}

The survey also collected data about the success of the conservation projects. Due to the lack of a standard evaluation system for TEK conservation outcomes in the literature, the survey could not give quantitative scales of the success assessment. Instead, it gathered the answers to the question of "How successful have these TEK conservation practices been in your location? Why"? Within the respondents who claimed conservation actions take place in their locations ( $n=112), 24 \%$ of the responses claimed definite success (wording includes very successful, successful and wonderful), $58 \%$ of them claimed limited success (wording includes somewhat successful, limited successful, partially successful and lightly successful), and 7\% claimed no success at all. The rest (11\%) claimed that it is too early or too difficult to assess.

\subsubsection{What has been successful and where?}

The successful cases were widely claimed from all regions, including 26 countries: Australia, Bolivia, Cambodia, Canada, China, Congo, Cyprus, Ecuador, India, Kenya, Laos, Malaysia, Marshall Island, Mexico, Mozambique, New Zealand, Pakistan, Panama, Peru, Solomon Island, South Africa, Tanzania, Thailand, Uganda, USA and Vietnam. Various conservation actions were claimed to be successful. According to the frequency that addressed to be successful by survey respondents, the most successful option is 
Research and documentation ${ }^{41}$, including independent research undertaken by outsiders and participatory research, in which local communities contribute to and share the results. Its success was reported by 22 respondents. For instance, in Wuikinuxv, Nuxalk, Kitasoo, Heiltsuk communities in the central coast of British Columbia of Canada, an intensive survey of key knowledge holders (usually elders) has been carried out. The survey focuses on collection of information on traditional harvest areas, changes in species populations over time, identification of important ecological features including: spawning/breeding areas, juvenile rearing areas, migration routes, place names and associated stories. As the researcher (pers. comm., 2010) claimed, "[This projects] has been quite successful, the survey results serve as an educational tool for [indigenous] youth. Information has been catalogued on GIS with audio/visual files and made accessible to the youth".

The second most frequently addressed successful option is community-based traditional lifeways programmes, such as programmes to increase indigenous language retention, cultural festival days, elders-youth camps, and canoe-building camps. For instance, in Loreto (Peru), a researcher (surv. resp., 2010) observed the establishment of an indigenous Maijuna federation which works to conserve both biological and cultural diversity. The federation initiated a language revitalisation programme, completed a wide variety of community-based biocultural conservation projects, which was targeting culturally significant plants, animals, etc. He assessed the projects as, "many of these projects have been very successful because they are community-based in nature and have been established and driven by the communities". The success illustrated by this researcher might more focus on the achievement of community participation. For long term, this is beneficial to TEK conservation overall.

The third most frequently listed successful action is community-based environmental conservation activities, including community-initiated conservation actions and community's participation in governmental-led conservation projects. For example, a conservation project in Mbirikani, Kajiado of Kenya employs indigenous community members to monitor carnivores and mitigate conflict in their communities. A researcher

\footnotetext{
${ }^{41}$ Please note there is a potential bias in this statement. This claim may be due to the fact that most respondents were researchers, so they saw their own action as successful.
} 
(surv. resp., 2010) assessed it as, "In developing the project, there was a high level of engagement with elders and the community in general which allowed forgotten TEK to be revived and incorporated into the project. By offering employment opportunities to these individuals specifically because of their traditional practices (rather than education), status and recognition has been attached to what is already a source of pride for many".

Another few options were also perceived as being successful, including: 1) indigenous media and informal learning, such as community newspapers, community radios, traditional healers workshop, indigenous museum, ethno-botanic garden and traditional medicinal herb nursery; 2) TEK commoditisation, including commoditise indigenous arts and crafts, showcase of traditional dance, songs and costumes, and ethno-tourism; 3) indigenous institutional development, including self-government and selforganisation; and 4) educational means, including include TEK in school curriculum and customary schools.

\subsubsection{Key reasoning for "successful"/Triggers to success perceived by the survey respondents}

1) The involvement of local community was addressed most frequently as the key factor that lead to conservation success. One third of the successful cases claimed the importance of community involvement. For example, when a researcher (surv. resp., 2010) assessed TEK documentation and ethno-tourism projects in Panama and Darien provinces of Panama, she stated, "I think the most successful projects have been those that have been able to engage the community as a whole and to get people excited about something that was lost".

In Jilantai, Inner Mongolia of China, a practitioner (Ma, pers. comm., 2010) concluded the reason for the success of a community-based integrated conservation and development project as, “... because the project is under the community care. The community regained certain resource rights, and formed their project management committee. This is a large success after 50 years practice of absolute government-run, top-down resource governance". 
2) Non-governmental external support to local-based projects, including organisational, financial and technical support is the second most common leading factor of TEK conservation success. As a researcher (surv. resp., 2010) from the Khunjerab national park of Northern Pakistan noted, there is a series of projects that aim to preserve traditional skills, to document the customary laws and to conserve wildlife species, which are supported by WWF-Pakistan. He assessed these projects as, "Under the constant support of WWF-Pakistan, most of the TEK projects have been successful, the price tag was attached with the traditional activities, and conservation of wildlife was linked with eco-tourism. The monetary benefits have placed good role in the sustainability of these activities".

A traditional canoe building training programme was claimed successful in Majuro of Marshall Islands (surv. resp., 2010.), "More traditional canoes are observed even in the urban capital; the NGO which does the training is fairly high profile and continues to receive funding".

3) The governmental recognition and support was perceived by two survey respondents as a trigger to TEK conservation success. In Thung Yai Naresuan Wildlife Sanctuary, Kanachanaburi of Thailand, village-level conservation groups to monitor and protect wildlife have been formed. These groups are led by traditional "Bokhu" knowledge holders. The reason for the success of the projects that summarised by a local researcher (surv. resp., 2010) is “... because these local conservation groups have attained some official recognition from the protected area. This is incentive for people to be involved, and many young people are involved".

In Mambasa, Province Orientale of Congo, the use of local knowledge of plants and animals, and social organisations has been incorporated into natural conservation projects. The projects are claimed by a community member (surv. resp., 2010) to be successful because, "... the use of traditional knowledge and social organisation has been supported by the governmental authority. Once the management plan was agreed by traditional authorities, all group members subscribe to it". 


\subsubsection{Reasoning for failure/Barriers to success perceived by the survey respondents}

More than half of the respondents stated the "limited success" or "not very successful" of the conservation actions. There are five common barriers addressed by the respondents despite the verbal variety used in different cases.

1) The most common barrier is the competing interests or cultural distraction from the outside world, including indigenous migration to town, increasing interests in Western employment and town-based lifestyle, and globalisation. A community elder from Ontario of Canada (surv. resp., 2010) stated, “We are worried about young peoples' greater involvement in town activities and less in bush activities. One result of this is that these young peoples lost their ability to talk Anishinaabe using specialized bush language. Even adults speak like children".

A researcher from Mexico (surv. resp., 2010) described the limiting factor of community-based TEK conservation activities as, “... One recurring problem is that often the most active members [of the community TEK conservation activities] end up migrating, when there is some problem - economic or otherwise - in their families".

A researcher from Hluhluwe, KwaZulu-Natal of South Africa reported, "There have been few attempts to conserve TEK practices in this location, as there is no value placed on retaining or transferring this knowledge. Communities now strive for westernised employment, lifestyles and money. I am not aware of any successful TEK conservation practices in this area".

2) The lack of governmental recognition and support is the second most common barrier addressed by the respondents. As a researcher (surv. resp., 2010) who works with several indigenous communities along Negro river and Purus River at Amazonas state of Brazil stated, "It has been relatively successful, unfortunately if all these initiatives don't have government support, they'll remain only as punctual experiences with lack of continuity". A researcher (surv. resp., 2010) from Chitwan National Park of Nepal claimed, "It has been successful but lack of adequate understanding among government authority is the problem". A researcher (surv. resp., 2010) from Maryland of USA described, "People in the area were happy that we were talking to them about 
their mushroom harvesting practices, and were interested in participating in management development and decision-making. Unfortunately the National Park Service seems more interested in documenting the stories than any type of adaptive management or co-management".

3) The lack of financial source and other supporting capacity is another main barrier that recognised by the respondents. For example, in Kahua, Makira ULawa Province of Solomon Islands, "Limited success due to 1): lack of continuity which cannot cope with the number of children who, once they finish kindergarten, leave the villages to get schooling elsewhere [regarding the community customary kindergarten programme]; 2) the organisations lack of funds and capacity to cope with high expectations from communities and aid donors - i.e. the organisation can't cope with these demands".

A similar claim was also made by a community member (surv. resp., 2010) in Matutuine District, Maputo Province of Mozambique, "[The projects] lacks sustainable financial resource. This is partially caused by recent political instability (Civil War)".

Other barriers to conservation success also include wider social, economic and political stress (e.g. social ignorance of TEK and indigenous values; national economic structure change; political instability), and the dominance of outside agencies projects, leaving no space for community initiatives and participation. As King and Cruikshank (2012) remind us, there is distinction between government-led community engagement and community-led community engagement. Government engaging community inherently contains power dynamics that can skew local involvement. It has been used as a means for government to achieve policy implementation and public acceptance (Eversole, 2011). It often addresses the needs of government, but gives communities a false sense of having been involved in the process (King and Cruikshank, 2012).

It has been noticed that there are significant similarities between the barriers to conservation success and TEK threats. This finding matches the real-world situations, in which TEK threats and conservation actions are always tightly interwoven. Any successful conservation design and implementation has to be based on comprehensive understanding of the complex TEK threats. 


\subsubsection{Reasoning for "hard to say"/other issues}

A significant number of respondents (11\%) claimed that the assessment of TEK conservation outcome is difficult, or impossible. The reasoning includes the lack of standard and procedure for assessing conservation outcomes, the perspective issue of assessing the success (e.g. success from a community's perspective, or from a researcher's perspective), and the long processing of the projects are hard to assess at this stage.

The results above demonstrate that the barriers towards TEK conservation factors operating at multiple scales, from the lack of financial and other capacity at the community level, to the lack of government recognition and support at regional and national levels, to wider social, cultural and economic influence from dominant societies. Accordingly, the success of TEK conservation requires a wide-range and multi-scale support, from community capacity and participation, to external organisational assistance and regional and state government's recognition and support. Every actor in this system is critically important.

\section{DISCUSSION AND CONCLUSION}

In this section, I will first discuss the principles for achieving effective TEK conservation actions, and then discuss the design of action plans through linking threats to actions. The limitation of the research will be illustrated, and the direction of future research will be pointed out.

\subsection{Principles for TEK conservation design}

As illustrated at the beginning of this chapter, international policy and legislation frameworks for indigenous rights and TEK protection have been in place for several decades. An ongoing key challenge in the field is how to transform these policy and legislation into real-world actions. Based on the analysis of triggers and barriers to TEK conservation success (section 2.4), it is clear that indigenous populations have been constantly challenged by internal change and limited capacity, and external influence 
and stress from multiple scales. This analysis provides a valuable message for TEK conservation designing and implementation that effective conservation actions have to incorporate internal capacity building within the indigenous populations, and external recognition and support from wider realms (DESA, 2009). These two approaches are equally vital to the success of TEK conservation.

Based on perception of the survey respondents and the results from literature review, I note three principles for conservation action design. First, an appropriate legislative framework at multiple levels is essential. As discussed under section 2.2.4, policy and legislation is a double-edged sword. On one hand, TEK-supporting policy and legislation may be one of the most effective mechanisms to protect TEK (e.g. Wavey, 1993; Zent, 1999); on the other hand, TEK-unfriendly policy and legislation may also be able to effectively cause TEK degradation (e.g. Wang, 2009; Xu and Melick, 2007). This statement has been proven by the survey and literature results of this research. While $10-11 \%$ of the literature and survey responses state that policy and legislation caused TEK loss in their cases, there are also about $8 \%$ of the survey responses and literature addressed the use of policy and legislation as a TEK conservation mechanism.

Policy and legislation at national and regional levels, acting in accordance with international standards, are critically important to assure the protection of TEK at the ground level (AHRC, 2008; Collings, 2009; Mauro and Hardison, 2000). The policy and legislation should recognise and protect indigenous land and resource rights, the rights of owning and using indigenous knowledge, and other human rights of indigenous people. The legislative framework should provide for:

1) the recognition, adoption and compliance with indigenous customary laws;

2) the full participation and engagement of indigenous people in decision-making or negotiations;

3) the protection of indigenous areas of significance, biodiversity and cultural heritage;

4) access to benefit-sharing through partnership between the private sectors and indigenous communities; and

5) non-discrimination and substantive equality (AHRC, 2008). 


\section{Second, genuine engagement of indigenous people in wider social-economic-}

political decision-making process is highly desirable. As discussed before, lower level organisations, especially the community level's participation in decision-making has been a major concern since the 1980s along with the occurrence of environment and resource governance decentralisation (Berkes, 2010; Larson and Soto, 2008; Lemos and Agrawal, 2006). Based on the essential goal of "bringing government closer to the governed and allowing people whose livelihoods and well-being would be affected by the decisions to have a say in those decisions" (Berkes, 2010: 489), effective user participation and problem solving at the lowest feasible level of organisation (usually community level), or the subsidiarity principle ${ }^{42}$ has been recognised as an essential part of good governance (Kooiman et al., 2005).

Three distinct justifications for such decentralisation have been recognised: 1) it can produce greater efficiencies because of competition among sub-national units; 2) it can bring decision-making closer to those affected by governance, therefore promoting increased participation and accountability; and 3) it can help decision-makers take advantages of more precise time-specific and space-specific knowledge about natural resources (Lemos and Agrawal, 2006). However, to achieve genuine good governance, the willingness and actual actions of power devolution from the authority system, the community's capacity of effective participation, and efficient communication across different levels of the institutions are equally important (Berkes, 2010).

The survey results demonstrate that community involvement is at the central importance of leading TEK conservation success, but is also claimed deficient in many cases. In order to achieve the genuine engagement of indigenous people, it requires:

1) a strong legislative and policy framework that recognise and protect indigenous rights (Zent, 1999);

2) free, priori and informed consent regarding the decision-making that may concern indigenous well-being and interests (AHRC, 2008);

3) social support, public awareness and recognition of indigenous rights and cultural distinctiveness (DESA, 2009);

${ }^{42}$ The principle emphasises that issues regarding resource management should be handled by the smallest, lowest, or least centralised authority that are capable of addressing the issues effectively. 
4) technical support, such as providing for information in indigenous languages (AHRC, 2008); and

5) mechanisms for solving disputes, such as an independent system of conflict resolution (AHRC, 2008).

To meet these requirements, governmental authorities, external organisations at multiple levels, and the general public are important actors in fostering support for indigenous communities. Genuine community engagement is an ongoing, long term process, in which actors across social and geographical scales equally share the responsibilities.

\section{Third, effective indigenous capacity building is vital to TEK and indigenous}

survival and revival. The indigenous capacity, as an essential factor of indigenous wellbeing, plays a fundamental role in the survival and development of indigenous populations (Simpson et al, 2003). Despite external stresses, communities can inherently retain the power to determine their own future. For example, many indigenous heritage sites have been nearly emptied by the migration process, even though the indigenous groups still hold sovereign over the land. The younger generations commonly decided to leave their communities is the main cause of this "empty land" phenomenon (pers. comm., 2010). As discussed under section 2.2.1, effective indigenous capacity building can be achieved through:

1) strengthening or rebuilding community social cohesion and community organisations through self-government and self-organisation;

2) motivating interests in traditional lifestyle and culture among community members though traditional lifeways programmes, community-based natural conservation activates, TEK commoditisation and indigenous education;

3) strengthening or rebuilding the community financial stewardship; and

4) strengthening and building indigenous alliances and partnerships

In order to achieve successful community development initiatives, true participation is needed. This can be driven both by necessity and awareness, and is dependent on knowledge and genuine skill acquisition processes. These processes can take 
considerable time and application, and thus require on-going support (Simpson et al., 2003).

The discussion of these three design principles reflects theory from the literature surrounding social-ecological systems. As Ostrom et al. (1999) point out, this nature calls for institutional arrangements that are linked both vertically (across hierarchical organisations; in this case, from community to external organisation and government) and horizontally (across regions; in this case the cooperation between different indigenous groups, and between different organisations and governmental agencies). This is because the complexity, uncertainty and non-linear behaviour of socialecological systems require a full range of knowledge and skills that is difficult for any single group or agency to possess (Berkes, 2010; Young et al., 2008). This indicates the necessity of having both bottom-up and top-down initiatives, as well as external support from other organisations.

As discussed in the Chapter One, many examples from the literature (e.g. Agrawal, 2002; Cruz García, 2006; Hill, 2004; Robert and Angela, 2004; Ross, 2002a; Zent and Maffi, 2009) and from the survey reveal that the unbalanced power relations between different layers of complex political-social-ecological systems, especially between the community and government levels, have obstructed genuine TEK conservation worldwide. The genuine participation of indigenous peoples in decision-making largely relies on policy, legislative and social support from the dominant societies (Adler and Bernstein, 2005; DESA, 2009).

\subsection{How to use TEK typology for conservation project design?}

Better conservation outcomes come from a better understanding of the action target (WWF, 2007); in this case it is the threats towards TEK. Chapter Three has provided a comprehensive overview and in-depth analysis of all direct and underlying threats to TEK. By linking conservation actions to specific threats, the logic of this analysis is to develop conservation strategies more precisely; therefore to enhance the effectiveness and efficiency of conservation actions (Galindo-Leal, 2001). 
Please note there are two personal decisions regarding the recommendations below: 1) research and documentation has been the most widely and frequently used mechanism in TEK conservation; and generally speaking, it needs to be further and wider applied. Thus, I will not recommend it specifically for any direct threat; and 2) in agreement with Swaderska (2001), Song (2010), and IIED (2006), the conventional Intellectual Property Rights system is recognised as non-preferable option for protecting TEK, thus is not recommended in any cases. Instead, I suggest the use of sui generis system in the role of protecting indigenous intellectual and cultural rights (refer to previous section).

\subsubsection{Direct threats and potential corresponding actions}

In this section, I will first present a comprehensive table that contains all direct threats and recommended conservation actions in response. Then, I will provide a detailed discussion of how to take action to deal with each threat with examples. The recommendations for corresponding actions are made at a basis of survey respondents' perception, data collected from multiple interviewees, recommendations in the literature and my own analysis. These recommendations might not be the best resolutions to the threats, but they will be a useful initial effort, which inspires further research on it.

Table 4.5 Direct TEK Threats and Potential Corresponding Conservation Actions

\begin{tabular}{|l|l|}
\hline \multicolumn{1}{|c|}{ Direct Threats } & \multicolumn{1}{c|}{ Potential Corresponding Actions } \\
\hline $\begin{array}{l}\mathbf{1}^{43} \text {. Loss of pathways of TEK } \\
\text { transmission }\end{array}$ & $\begin{array}{l}\text { 2. Community-based TEK conservation } \\
\text { 3. Education and awareness } \\
\text { 4. Policy and legislative support }\end{array}$ \\
\hline 1.1 Loss of traditional language & $\begin{array}{l}\text { 2.1 Traditional lifeways programmes } \\
\text { 3.1.1 Teach indigenous language in formal education } \\
\text { system } \\
\end{array}$ \\
& 3.2 Customary education \\
& 4. Policy and legislative support \\
\hline
\end{tabular}

\footnotetext{
${ }^{43}$ The numbering in this table is consistent with the numbering in the TEK threat and conservation action typologies.
} 


\begin{tabular}{|c|c|}
\hline $\begin{array}{l}\text { 1.2 Influence induced by formal } \\
\text { educational system }\end{array}$ & $\begin{array}{l}\text { 2.1 Traditional lifeways programmes } \\
\text { 3.1 Include TEK in formal education } \\
\text { 3.2 Customary education } \\
\text { 3.3 Indigenous media and informal learning }\end{array}$ \\
\hline $\begin{array}{l}1.3 \text { Younger generations' absence from } \\
\text { the traditional community }\end{array}$ & $\begin{array}{l}\text { 2.1 Traditional lifeways programmes } \\
\text { 3.1 Include TEK in formal education } \\
\text { 3.2 Customary education } \\
\text { 3.3 Indigenous media and informal learning }\end{array}$ \\
\hline $\begin{array}{l}\text { 1.4 Influence induced by dominant } \\
\text { societies \& reduced incentive and interest } \\
\text { in TEK }\end{array}$ & $\begin{array}{l}\text { 2.1 Traditional lifeways programmes } \\
\text { 2.3 TEK commodidation } \\
\text { 3.1 Include TEK in formal education } \\
\text { 3.2 Customary education } \\
\text { 3.3 Indigenous media and informal learning }\end{array}$ \\
\hline $\begin{array}{l}\text { 2. Change of traditional livelihood } \\
\text { practices }\end{array}$ & $\begin{array}{l}\text { 2. Community-based TEK conservation } \\
\text { 4. Policy and legislative support }\end{array}$ \\
\hline $\begin{array}{l}2.1 \text { Reduced land-based and sea-based } \\
\text { activities }\end{array}$ & 4. Policy and legislative support \\
\hline $\begin{array}{l}2.2 \text { Reliance on modern products and/or } \\
\text { technologies }\end{array}$ & $\begin{array}{l}\text { 2.1 Traditional lifeways programmes } \\
\text { 4. Policy and legislative support }\end{array}$ \\
\hline $\begin{array}{l}2.3 \text { Use of westernised primary } \\
\text { production systems }\end{array}$ & 2.1 Traditional lifeways programmes \\
\hline $\begin{array}{l}\text { 3. Change of traditional religion and } \\
\text { beliefs }\end{array}$ & $\begin{array}{l}\text { 2. Community-based TEK conservation } \\
\text { 3. Education and awareness } \\
\text { 4. Policy and legislative support }\end{array}$ \\
\hline $\begin{array}{l}3.1 \text { Conversion to other dominant } \\
\text { religions }\end{array}$ & None \\
\hline 3.2 Loss of traditional belief & $\begin{array}{l}\text { 2.1 Traditional lifeways programmes } \\
\text { 3.1 Include TEK in formal education } \\
\text { 3.2 Customary education } \\
\text { 3.3 Indigenous media and inform learning }\end{array}$ \\
\hline $\begin{array}{l}\text { 4. Change of environment and natural } \\
\text { resources }\end{array}$ & $\begin{array}{l}\text { 2. Community-based TEK conservation } \\
\text { 4. Policy and legislative support }\end{array}$ \\
\hline $\begin{array}{l}4.1 \text { Change of physical location and } \\
\text { environment }\end{array}$ & $\begin{array}{l}\text { 2.1 Traditional lifeways programmes } \\
\text { 2.2 Environmental conservation activities } \\
\text { 4. Policy and legislative support, esp. indigenous claims }\end{array}$ \\
\hline 4.2 Resource degradation & $\begin{array}{l}\text { 2.2 Environmental conservation activities } \\
\text { 4. Policy and legislative support }\end{array}$ \\
\hline
\end{tabular}




\begin{tabular}{|l|l|}
\hline 5. Loss of traditional rights & $\begin{array}{l}\text { 1. Capacity building } \\
\text { 2. Community-based TEK conservation } \\
\text { 4. Policy and legislative support }\end{array}$ \\
\hline $\begin{array}{l}5.1 \text { Loss of traditional land and/or land } \\
\text { tenure }\end{array}$ & $\begin{array}{l}\text { 1.1 Institutional development } \\
\text { 4. Policy and legislative support, esp. indigenous claims }\end{array}$ \\
\hline 5.2 Loss of traditional land use & $\begin{array}{l}2.1 \text { Traditional lifeways programmes } \\
\text { 4. Policy and legislative support }\end{array}$ \\
\hline 5.3 Loss of access to traditional resources & 4. Policy and legislative support, esp. sui generis \\
\hline 6. Loss of traditional institutions & $\begin{array}{l}\text { 1. Capacity building } \\
\text { 2. Community-based TEK conservation }\end{array}$ \\
\hline $\begin{array}{l}\text { 6.1 Replacement of traditional resource } \\
\text { governance }\end{array}$ & $\begin{array}{l}1.1 \text { Institutional development } \\
4 . \text { Policy and legislative support, esp. indigenous claims }\end{array}$ \\
\hline $\begin{array}{l}\text { 6.2 Loss of other aspects of traditional } \\
\text { institutions }\end{array}$ & $\begin{array}{l}\text { 1.2 Alliance and partnership development } \\
2.1 \text { Traditional lifeways programmes }\end{array}$ \\
\hline
\end{tabular}

\section{1) Loss of pathways of TEK transmission and corresponding conservation actions}

As some scholars (e.g. Eyssartier et al., 2008; Ohmagari and Berkes, 1997) and many survey respondents point out, the loss of TEK transmission is the most urgent threat to TEK worldwide. It thus becomes the most immediate challenge to TEK conservation actors in the field. Overall, to deal with degraded TEK transmission, options of community-based TEK conservation activities, education and awareness and policy and legislative support may provide resolutions.

According to the survey results, there are two major causes of TEK transmission degradation: 1) younger generations' disinterest in traditional knowledge and practices; and 2) the access and reliance on modern products and technologies. They both reduce the incentives of learning TEK within indigenous populations. Community-based TEK conservation, such as traditional lifeways programmes and TEK commoditisation, can provide a chance for indigenous populations to apply and appreciate their traditional practices and culture, especially with indigenous youth, who still live in the community. It therefore improves the possibility of TEK transmission.

Since disinterest in, and lack of inherent value of TEK by indigenous youth may be 
caused in part by Westernised formal education (e.g. Benz et al., 2000; Ellen, 2007; Godoy et al., 2008; Lizarralde, 2001; Voeks and Leony, 2004; Zent, 2001), the use of educational and communicational means becomes vital mechanisms to battle with the degradation of TEK. The inclusion of TEK in the formal education system may increase the general interest and recognition of TEK amongst the youth, both indigenous and non-indigenous. It then will increase the appreciation of the indigenous identity and traditional lifeways, and trigger the interest in learning it within the indigenous youth. Customary education is a more comprehensive educational mechanism when compared with including TEK in formal education. This is because customary education is a learning process for the indigenous, by the indigenous (May, 1999). Within this process, transmitting traditional knowledge and culture is a fundamental mission.

Indigenous media and informal learning has a wider target with both indigenous populations and the general public. It advocates TEK through information sharing in an interesting way and through various public educational means, such as indigenous museums, indigenous botanical gardens and community radio stations. These programmes are able to reach and influence wider populations when compared with the other two educational means discussed above (Colfer, 2000).

Policy and legislative support may not directly affect TEK transmission, but it determines whether TEK can be included in the formal education system; it influences the dominant society's attitude towards indigenous people and their culture (Ajzen and Fishbein, 1980). It therefore indirectly influences indigenous youth's interest and confidence in their traditional practices and culture, and ultimately influences the TEK transmission rate. This is a typical example to demonstrate that in each case there are key actions needed to deal with direct threats and underlying causes simultaneously.

In Table 4.5, I recommended different specific actions for dealing with specific subcategories of TEK threats. For example, if the loss of indigenous language is the major issue in one location, the conservation actor may concentrate on language retention programmes. To achieve language retention, the action options may include following: 1) teach indigenous language and TEK in formal schools. This action can increase the recognition and proud of indigenous language and TEK amongst indigenous youth, as 
well as increase their indigenous language skills; and 2) establish or promote community-based customary educational institutions. By applying this action, the indigenous youth will have a chance to learn from the elders about traditional knowledge, skills, and worldviews, which are carried by their indigenous language; 3) traditional lifeways programmes, such as elder camps, youth challenge and community cultural days. By organising these activities, a community or a conservation team can increase youth's interest in TEK and traditional language; and 4) law and policy that support, encourage and promote indigenous language and cultural practices will be a significant supporting force to indigenous language retention.

\section{2) Change of traditional livelihood practices and corresponding conservation actions}

To directly deal with this threat, there are two key options - community-based TEK conservation and policy and legislative support. There are two types of the change in traditional livelihood practices: 1) enforced change by reduced resource and land availability; and 2) voluntary change due to the access and reliance on modern products, technologies, and Westernised production system. The first type can be dealt through indigenous land and other rights claims. The foundation of these indigenous claims is legislative and policy support, including international, national and regional levels.

The second type can be dealt through traditional lifeways programmes. For instance, if the main threat in a community is the reliance on modern products (e.g. progressed foods and modern tools), traditional lifeways programmes can generate opportunities for indigenous people, and may especially help the youth to learn how to make a living from natural resources (e.g. forest food sources, traditional tool making by using natural materials). It therefore promotes the use of traditional practices and skills.

\section{3) Change of traditional religion and beliefs and corresponding conservation actions}

The change of traditional religion and beliefs can also be defined into two types: 1) the conversion to other dominant religions, which is often enforced by a dominant cultural force (e.g. through missionary during the colonisation processes); and 2) loss of 
traditional beliefs through contact with other cultures during the modernisation or westernisation processes. To deal with the loss of traditional religions and beliefs, community-based TEK conservation and education and awareness can be used as main conservation mechanisms.

For example, if an indigenous group is facing the loss of their traditional beliefs in sacred landscape and spirits, conservation actors can design a series of traditional lifeways programmes, in which traditional ceremonies and rituals will be presented, and traditional beliefs can be understood and appreciated.

Education and awareness is another effective mechanism. Including traditional cultural beliefs in formal schools and building customary schools to pass the traditional ideology and worldviews can promote the understanding and interest in traditional beliefs within indigenous youth. In order to communicate with wider indigenous population and gain their attention and interests in traditional beliefs, conservation actors can create informal learning projects, such as exhibition of traditional culture, and performance of traditional rituals.

\section{4) Change of environment and natural resources and corresponding conservation actions}

The change of environment and natural resources can be further defined into two subcategories: 1) the change of physical location and environment caused by enforced or voluntary relocation or migration; and 2) resource degradation caused by natural factors (e.g. climatic fluctuation) or non-sustainable human activities. To deal with these sub-threats, community-based TEK conservation and policy and legislative support can be used as immediate responses.

For example, if the main threat is change of physical location caused by enforced relocation, the affected indigenous group should use indigenous claims to regain their traditional land rights. Meanwhile, the group can carry out traditional lifeways programmes, such as traditional festival days, to revitalise their traditional practices. 
If the main threat is resource degradation caused by human activities, the direct actions may be establishing and implementation of conservation legislation and policy, which provide a legal frame and policy support for environmental conservation activities. The environmental conservation activities may be initiated by the indigenous community by using their traditional knowledge, practices and institutions; or organised by external organisations, such as governmental agencies, research institutions and nongovernmental organisations, with local community's participation.

\section{5) Loss of traditional rights and corresponding conservation actions}

Loss of traditional rights is the most fundamental threat to the survival of TEK and indigenous culture. It can be further divided into three sub-threats: 1) loss of traditional land or land tenure; 2) loss of traditional land use; and 3) loss of access to traditional resources. To deal with these threats, conservation actors can search through a wide range of actions, including capacity building, community-based TEK conservation, and policy and legislative support. The capacity building and community-based TEK conservation aim to strengthen community social cohesion and the ability to cope with external disturbance; law and policy (including indigenous intellectual property rights systems) aim to provide external support to protect indigenous rights.

For instance, if the main threat in a community is loss of the ownership of traditional land, the most direct resolution is to claim the land back. For doing so, the relevant legislative and policy support is essential. Meanwhile, the indigenous population needs to strengthen their institutional capacity, such as self-government and self-organisation, to be able to deal with the long and challenging processes of land claims.

\section{6) Loss of traditional institutions and corresponding conservation actions}

Loss of traditional institutions has been recognised as a direct threat to TEK at a limited level in the literature and by the survey respondents. However, my own research (Tang and Gavin, 2010; next chapter) and a number of studies (e.g. Berkes, 2004, 2010; Young, 2006) show that, the institutions play an important role in maintaining and revitalising traditional knowledge and cultural systems. Institution contains two major components: the social organisation and the customary norms and rules. The loss of traditional 
institutions can be further defined into two sub-threats: 1) replacement of traditional resource governance; and 2) loss of other aspects of traditional institutions. To deal with these threats, capacity building, community-based TEK conservation, and policy and legislative support are recommended. Similar to the actions responding to the previous threat, capacity building and community-based TEK conservation aim to develop the internal capacity of a target community to cope with external disturbance; Law and policy aim to provide external support to community's efforts of maintaining and revitalising traditional institutions.

For instance, if the main threat to an indigenous community is the replacement of traditional resource governance, the most direct conservation action is to re-claim the resource ownership and rights to organise resource governance. For doing so, the community needs to be equipped with internal capacity of self-governance, and external legislative and policy support.

The above discussion is merely recommendations. In real-world cases, the situation would be much more complicated, in which multiple factors that affect conservation decision-making, such as capacity limitation, cultural preference, financial restrains, and social-economic-political influences. TEK conservation design should be a dynamic and adaptive process. Meanwhile, it is very important to bear the various underlying threats to TEK in mind at the same time. The actions that target specific direct threats may mitigate TEK degradation at a certain extent in a short term; in order to achieve longterm effectiveness of TEK conservation, underlying causes of TEK degradation also have to be dealt with.

\subsubsection{Underlying threats and corresponding actions}

Due to the complexity of the interrelationship between direct and underlying threats, linking underlying threats and conservation actions is beyond the target of this research. Below, based on the examples from the survey and my own analysis, I only provide a general idea of what conservation action should be considered under each underlying threat. 
Table 4.6 Underlying TEK Threats and Potential Corresponding Conservation Actions

\begin{tabular}{|c|c|}
\hline Underlying threat & Potential Corresponding Actions \\
\hline \multirow[t]{2}{*}{ Governmental policy and legislation } & Policy and legislative support \\
\hline & Education and awareness \\
\hline \multirow[t]{2}{*}{ Contacts with other cultural groups } & Community-based TEK conservation \\
\hline & Education and awareness \\
\hline \multirow[t]{2}{*}{ Influence of the outside market } & Capacity building \\
\hline & Community-based TEK conservation \\
\hline \multirow[t]{3}{*}{ Colonisation } & $\begin{array}{l}\text { Policy and legislative support, esp. indigenous } \\
\text { claims }\end{array}$ \\
\hline & Community-based TEK conservation \\
\hline & Capacity building \\
\hline Relocation & $\begin{array}{l}\text { Policy and legislative support, esp. indigenous } \\
\text { claims }\end{array}$ \\
\hline \multirow[t]{2}{*}{ Marginalisation by dominant societies } & Education and awareness \\
\hline & Capacity building \\
\hline War and military occupation & $\begin{array}{l}\text { Policy and legislative support, esp. } \\
\text { indigenous claims }\end{array}$ \\
\hline Indigenous population decline & None \\
\hline \multirow[t]{2}{*}{ Migration } & Capacity building \\
\hline & Community-based TEK conservation \\
\hline Economic development pressure & Capacity building \\
\hline
\end{tabular}

I note there is a significant literature gap that future research may fill by identifying the specific linkages between underlying threats and corresponding actions. This work could be used to incorporate it with the linkages between direct threat and conservation actions and form a combined system, which can effectively direct the conservation practices in the field. 


\subsection{Limitation \& Recommendations for future research}

This chapter shares several limitations with Chapter Three, including the limited sample size, uneven distribution of responses from different countries and regions, and uneven distribution of responses from different target groups (please see detailed discussion in Chapter 3, section 4.4). Based on these research limitations, I have made several recommendations for future research.

\section{1) There is an urgent need to undertake more studies that focus on TEK}

conservation options, both case studies and review studies. More research will create rich data and form databases. Up to date, the understanding of TEK conservation options is incomplete, their applicability and efficiency is largely unknown. This causes difficulty to practitioners and decision-makers when making action plans for TEK conservation.

2) There also is an urgent need to develop a standard procedure for TEK conservation action assessment. A standard system is essential for any research discipline, which enables data collection and data analysis to be more systematic and comparable. In the practice, a standard procedure will facilitate monitoring and analysing of conservation actions, and making adaptations accordingly. It therefore will enhance the conservation effectiveness.

3) Due to data limitations, this research could not identify the linkages between underlying threats and corresponding conservation options specifically. It requires further research to fill this gap. Underlying threats is a large and complex system, which cover a wide range of disciplines, including political, social, economical and ecological sciences. To deal with these threats, it requires multi-disciplinary knowledge and methodologies. To fully understand the dynamics between underlying threats and direct threats, and to develop a systematic action plan for TEK conservation, it will need a holistic, pluralistic research approach and a multi-disciplinary research team. 


\subsection{Conclusion}

The values and the declining trend of TEK both contribute to the urgency of TEK conservation. The protection of TEK has been an international agreement since the early 1990s. Even though several policies have supported indigenous rights and TEK protection across the globe, the transferral of these policies into real-world actions has remained a major challenge to indigenous communities, governmental agencies, practitioners and academic researchers.

Based on a global survey ( $n=137)$, its follow-up interviews $(n=46)$ and a literature review ( $\mathrm{n}=152)$, this research reviewed TEK conservation actions, both applied and theoretical. It then developed a classification system of TEK conservation options, which includes five categories: indigenous capacity building, community-based TEK conservation activities, education and awareness, policy and legislative support, and research and documentation. Under each category, there are multiple sub-categories and examples to demonstrate how the conservation actions can be carried out. This TEK conservation typology fills an important literature gap.

According to the survey results, the majority $(82 \%, \mathrm{n}=112)$ of the survey respondents claimed that TEK conservation actions have been undertaken in their locations. Within these actions, the three most frequently used options are research and documentation (39\%), community-based TEK conservation activities (25\%), and education and awareness (16\%). Capacity building has been claimed by $12 \%$ of the despondences, policy and legislative support claimed by $8 \%$ of the responses.

Conservation outcomes were variable. Twenty four per cent of the survey respondents claimed definite success, $58 \%$ claimed limited success, $7 \%$ claimed no success at all, and the rest $11 \%$ claimed that it is too early or too difficult to assess. The key leading factors to conservation success perceived by the survey respondents are: 1) the involvement of local community; 2) external support to local-based projects; and 3) the governmental recognition and support. 
From the survey results and literature review, this research then summarises three principles for achieving effective TEK conservation: 1) an appropriate legislative framework at multiple levels; 2) genuine engagement of indigenous people in wider social-economic-political decision-making process; and 3) effective indigenous capacity building. A successful TEK conservation regime should incorporate internal motivation and capacity building from within the indigenous population, and external recognition and support from wider realms.

This research also establishes and presents the linkages between direct TEK threats and corresponding conservation actions with given examples. This is the first time that this task has been attempted in the literature. For future research direction, this research recommends to: 1) undertake more studies that focus TEK conservation options, both location-specific cases studies and review studies with cross-project and cross-location scales; 2) develop a standard procedure of TEK conservation action assessment; and 3) undertake more studies to identify the linkages between underlying threats and corresponding conservation options specifically. 


\section{CHAPTER FIVE}

\section{CASE STUDY}

\section{INTRODUCTION}

The previous two chapters provided an overall analysis of global threats to TEK and all possible TEK conservation actions. In this chapter, I will present a case study that investigates the institutional dynamics between governmental authorities and community organisations with a special focus on the impact of government policies on local level TEK and community institutions in Inner Mongolia, China. The study will illustrate how government institutions can be considered as a threat to TEK, but also how these institutions are dynamic and over time may turn into a conservation tool that supports TEK. After providing the detailed context of this case study, in the next chapter I will use this case study as an example to demonstrate how to apply the TEK classification systems (developed in Chapter $3 \& 4$ ) to real-world situation analysis.

\subsection{What are institutions?}

The academic use of the term institutions has become widespread in multiple disciplines of social science in recent years, including philosophy, sociology, politics, institutional economics, and human geography (Hodgson, 2006). There is no unanimity in the definition of this concept. However, in the field of economics and commons management, a commonly quoted definition was developed by North (1991:97): "[Institutions] are humanly devised constraints that structure political, economic and social interactions. They consist both informal constraints (sanctions, taboos, customs, traditions and codes of conduct), and formal rules (constitutions, laws and property rights)". According to Ostrom (1999:51), institutions are "the set of rules actually used (working rules or rulein-use) by a set of individuals to organise repetitive activities that produce outcomes affecting those individuals and potentially affecting others". In this sense, institutions are a set of formal and informal rules that shape interactions of humans with others and of humans with nature (Agrawal, 2003). 
However, in many applied studies, institutions are also referred to as the social organisations that participate, act and/or manage a focal resource system (Hodgson, 2006). There are endless disputes over the meanings between two key terms institutions and organisations (Hodgson, 2006). In this case study, by combining various definitions from different sources, institutions represents a broad meaning that includes the social organisation, the rules of resource use and management, and the action of developing these rules.

\subsection{Why do traditional institutions matter?}

As discussed in Chapter One (section 3.1), TEK is a dynamic and complex system which is comprised of four levels, including knowledge of species and landscape/seascape, resource use and management systems, social institutions and worldviews. Social institutions are a critical component of TEK. As North (1990) argues, knowledge of species and the environment, and resource management systems do not function in isolation; rather, they are embedded in institutions and local social norms. Many scholars point out that institutional arrangements are vital for effective natural resource management, biodiversity conservation and community empowerment (e.g. Argawal and Gibson, 1999; Berkes, 2007; Hanna et al., 1996; Ostrom, 2005). This is because institutional arrangements shape management strategies and affect multiple stakeholders in a given resource system (Anderies et al., 2004). As Brondizio et al. (2009) emphasise, institutions play a significant role in facilitating cross-scale resource and environmental governance. A multi-level, collaborative set of institutions may be the only effective means of managing multi-level social-ecological resource systems (PahlWostl, 2009).

The value and significance of traditional institutions has been recognised and emphasised in recent years along with the development of resource governance reform worldwide. Within this process, democratic political structures have been promoted, insistence on participation has increased, and in particular the popularity of community-based management projects has grown (e.g. Berkes, 2004, 2010; Young, 2006). There are many attempts to explore or develop community-based resource and conservation institutions. While some newly built community-based institutions have 
successfully managed their resources over the last few decades (e.g. Ostrom, 2005, 2007; Velez, 2011), most of the traditional institutions are based on community level practices, but evolved over a much longer period. Apart from leading to successful resource management, more importantly, conservation and revitalisation of traditional institutions can empower indigenous communities and enhance their share in decisionmaking (Agrawal and Gibson, 1999).

Based on my previous research in the Alashan region of Inner Mongolia (Tang, 2007; Tang and Gavin, 2010), I found the most threatened aspects of local TEK is the social institutions, especially the traditional organisations, which are responsible for creating and implementing rules of resource system boundaries and access, resource allocation, input, conflict resolution, and punishment. The research results showed that knowledge of how these organisations function still exists especially among elders; however, these traditional organisations have not been in place for at least 35 years (Tang and Gavin, 2010). As a result, the knowledge of how they function, and the ability and willingness of community members to embrace these institutions may not last much longer. This indicates that the knowledge and practice of traditional institutions has reached a threshold point, in which permanent loss will be inevitable if no immediate conservation action is taken (Walker and Meyes, 2004). The situation in this study is unlikely to be unique; rather, the loss of traditional institutions has been observed from various locations across the globe (e.g. Kipuri, 2009; Ostrom, 2009; Stavenhagen, 2005).

\subsection{Why study institutional dynamics in Inner Mongolia, China?}

There is a significant research gap in understanding of the necessity and approaches of cross-scale institutional arrangements in China. This is because the centralised government-controlled approach has been recognised as the "best", and the only way to run resource management by the authorities (Wang, 2009). Based on this perception, in association with the political sensitivity of challenging the current centralised power structure, the study of government policy's effects on local institutions and alternative resource management regimes is largely lacking in China. The influence of local level institutions has long been undermined by the decision-makers from the central governmental authorities (Ao, 2003, 2005). Allowing or encouraging non-governmental 
institutions has been a contested issue.

The importance of cross-scale institutional arrangements in achieving effective resource governance is addressed in Chapter One (see section 5.2). According to the most recent observation and reviews (e.g. Anderies et al., 2004; Berkes, 2003, 2007), a pure community-based approach cannot achieve success alone. Positioned in a complex, interdependent social-ecological system, community level institutions are severely affected by the actions of other levels of the system; such impacts can be either positive or negative (Berkes, 2010). The interactions between traditional community institutions and other levels of the institutional system are a critical determinant of the trajectory of TEK. Therefore, positive input from other levels is needed to support the maintenance and revitalisation of traditional institutions at the community level. As Harris (2007) reminds us, the complexity of social-ecological resource systems require matching multi-scale governance, in which the cooperation across levels and across space can be achieved.

\subsection{The Cooperative movement and the Cooperative Organisation Law}

\subsubsection{The cooperative movement and issues}

From the 1980s, along with the implementation of the Economic Opening Up Policy ${ }^{4}$, economic development has occurred much faster in urban areas than in rural regions of China. This has led to increased inequality in the country, and has marginalised rural populations both economically and socially. For example, in 2008 the average annual income per person in urban areas was NZ\$3156, while for the rural population it was NZ\$952 (The National Bureau of Statistics China, 2010). Rapid urban development requires a huge number of labourers from rural areas. Living in a city or town and earning higher incomes has become a major attraction for the rural population, especially among the youngsters. In 2006 alone, 13,181,000 rural people moved to urban areas for jobs (The National Bureau of Statistics China, 2008). However, due to the lower levels of education and a lack of relevant skills, many rural-to-urban migrants have ended up in the cheap labour market, such as construction sites, domestic service,

\footnotetext{
${ }^{44}$ It refers to the programme of economic reforms called "socialism with Chinese characteristics" in the 1980s. The reform is characterised with applying market-based principles into the Chinese economy.
} 
and restaurant labour. It is common for them to have lower salaries and lower living standards when compared with those originating from urban environments (Han, 2009).

Lower average annual incomes in rural regions drive further migration towards cities and towns which further degrades the economy of rural areas. To combat this disparity, farmers have been taking collective actions to form their own groups and assist them in meeting the challenges in the market economy. Up to 2006, more than $38,700,000$ rural households were involved in such organisations, which accounted for $13.8 \%$ of the total rural population in China (Guo and Song, 2009). Most of the organisations concentrate on crop farming, livestock farming and other agricultural production with a recent trend of developing eco-cultural tourism and traditional handcrafts trading (Young et al., 2006). Although China's transition to a market economy is approaching a more mature stage, the development of farmers' associations is still in its infancy (World Bank, 2006).

In Inner Mongolia, community-initiated herders' organisations emerged in the late 1990s. After a few decades of unsustainable use of grassland resources, the local ecosystems have experienced severe degradation (Da, 2008; Jiang et al., 2006; Tang and Gavin, 2010; Williams, 1996, 2002). As a result, individual households have become extremely vulnerable to any environmental and social changes (Ao, 2003). Traditionally, community collective institutions enabled herders to share the environmental risks and enhance social and ecological resilience (Ba, 2008). However, the traditional institutions have been suppressed or abandoned (often under government influences) over decades. In response to increased vulnerability to environmental changes and the loss of traditional institutions, some communities started to form organisations in which they can carry out traditional practices, such as seasonal herding rotations and sharing production equipment. These organisations mainly focus on organising production and trading activities at the community level, as well as preserving traditional cultural heritage, such as folk song recording programmes and community museums for Mongolian artifacts (Ba, 2010). Members of such organisations take collective actions in market exchanges, and share environmental and market risks.

However, given the political and economic history of China, in which absolute governmental control has long been practiced, government interventions have been a 
major issue for lifes at the community level. In order to avoid any potential governmental intervention, these organisations had been seeking lawful entitlement. Prior to October 2007, these organisations were only able to register as a non-profit organisation with the Civic Affairs Bureau to gain lawful status. However, traditional production activities, such as herding and livestock trading, were considered as profitmaking activities, and were therefore not allowed under the title of a non-profit organisation (Ba, 2008; Wang, 2009). The purpose underlying this regulation is the intention of absolute economic control by the government, and it has proven effective (Da, 2008).

\subsubsection{The Cooperative Law}

With a goal of narrowing down urban-rural income disparities in China and achieving a certain level of economic and social equity between urban and rural, the State Council (Document No.1, 2006) urged the progress of a national law to guide and support farmers and herders to form economic cooperative organisations in China. In October 2007, the China Farmers' Professional Cooperative Organisation Law (Cooperative Law) was enacted by the Chinese People's Congress Standing Committee. The law requires that all provisional and local level governments are responsible for implementing the law and promoting the development of farmers and herders' Cooperative Organisations (abbreviated as cooperatives below). As some Chinese scholars illustrate, the development and implementation of the Cooperative Law is also a result of the central government observing and receiving the feedback of the cooperative movement, mainly indirectly from lower levels of the governmental system (e.g. Wang, 2009; Xu, 2007).

The law supports and promotes cooperative organisations from two main perspectives. First, it recognises the legal corporation status of cooperatives. Once a cooperative is registered with the local Industry and Commerce Bureau, the organisation will be entitled as a corporation that has lawful rights to run its own economic activities. For example, Article Six of the law states that the law protects cooperatives and their members' legitimate rights and interests, which are inviolable by any individuals, organisations and governmental agencies. Second, the law provides policies that assist the development of cooperatives. It establishes credit, financial, taxation and 
registration systems that are conducive to the development of cooperatives. For example, in most of the cases so far, the cooperatives have been treated with concessions, including registration fee exemptions and tax reductions or exemptions.

The law requires that the local and regional governments are responsible for supporting certain types of cooperative activities, including technology training, information services, and infrastructure development. It also encourages financial institutions, both authorities and corporations, to offer loans to cooperative organisations for their productions and trading activities.

As discussed above, the fundamental goal of the law is to enhance rural economic capacity and overall social equity in China. Under this goal, the law may create space for rural capacity building in economic, institutional and political domains. Within a cooperative organisation, participating households are able to make decisions regarding resource use and other production practices. In this case study, herders can carry out their traditional herding practices and possibly revitalise the traditional institutions behind these practices. A legal status and policy support coupled with economic assistance (e.g. a tax deduction) would potentially promote the formation and development of such organisations, and empower the indigenous communities.

\subsection{Aim and Objectives}

The overall aim of this case study is to explore the possibilities of revitalising traditional practices and traditional institutions under historical governmental policies and the new opportunity that the Cooperative Law creates.

Five objectives are developed to achieve the research aim:

1. To investigate the historical governmental policies and their effects on Mongolian traditional practices and traditional institutions over the last 50 years.

2. To examine the implementation of the Cooperative Law at the local level.

3. To investigate the cooperative activities in the focal communities.

4. To examine the triggers and barriers to the cooperative activities.

5. To explore the opportunities of revitalising Mongolian TEK created by the Cooperative Law and the current cooperative movement. 


\section{THE RESEARCH SETTING}

This case study research is mainly based in Bayin Wenduri gacha ${ }^{45}$, in Xi Wuzhu Muqin Qi46 0 Inner Mongolia (see the map of the research location in Chapter 2, section 4.2.1, Figure 2.1). The site is a typical local village in the region in terms of its land area, population size, population structure, and economic activities. However, a special feature of this village is the existence of a cooperative organisation - Aolin Baolige Pastoralism Cooperative ${ }^{47}$, which was formed in 2009. The site selection was also a result of my previous research in Inner Mongolia. One of my close contacts in western Inner Mongolia, where I conducted my Masters research (Tang, 2007), introduced me to a community member in Bayin gacha as my research stay host. My host successfully introduced me to the community through his family and found a translator (MongolianChinese) for me.

The total territory of Bayin gacha is $380 \mathrm{~km}^{2}$ (38,000ha). The official record of the village population was 396 within 96 households (Ao, pers. comm., 2010). However, the actual number of the accessible households during the research period was $69(n=276)$. Thirteen households were removed to town due to mining activities; eight households lived in town or other cities because they found jobs there; and another six households were herding and visiting their relatives in other areas. The average pasture per household was 3.3-4 $\mathrm{km}^{2}$ (330-400ha, the average size of a household was 3-4 persons). The average annual income per household was NZ\$ 3,000 (about NZ\$ 750/person). The total number of livestock in the village was 3,200, in which sheep took the absolute majority $(n=2,800)$, while goats, cows and horses contributed another 400 to the total.

In order to conserve the grassland ecosystem, the central and local governments have enforced a policy of livestock reduction since 2000 with a standard of 1.5 ha per sheep unit, which means every sheep should be sustained by 1.5 ha of pasture. One goat counted for two sheep units and one cow or horse counted for five sheep units. The actual sheep to pasture ratio in the village was $0.8 \mathrm{ha} / \mathrm{sheep}$ in 2010 . The penalties for exceeding the standard included fines (NZ\$ 6 per exceeding sheep) and confiscation.

\footnotetext{
${ }^{45}$ Gacha means village in Mongolian. It will be abbreviated as Bayin gacha below.

${ }^{46}$ Literally means the western Wuzhu Muqin Banner. It will be abbreviated as Xiwu Qi below.

${ }^{47}$ It will be abbreviated as Aolin cooperative below.
} 
The village members constantly faced potential fines and confiscation. To avoid financial loss, community members often moved livestock around to hide them from the inspections that were carried out by the local authority; and herders called this strategy "being guerrillas". In most of the cases, the village leaders were willing to provide information of upcoming inspections and help herders to escape from penalty. As a village leader explained $\left(\mathrm{Ao}^{48}\right.$, interview, 2010), "the penalty will not save the grassland, but only generate further poverty of herders, and more conflict between the authority and the community". He also pointed out that "village leaders are herders themselves, and have strong sympathy towards other village members". This reflects a common disloyalty to government regulations by village members. As many village interviewees pointed out, the government decisions on pasture management did not take herders' knowledge and opinions into account. Many of these decisions were not consistent with the traditional wisdom of resource management in grassland ecosystems. They therefore did not have faith in these regulations.

Pastoral production in the community was based on individual households since the privatisation policy was introduced in the 1980s. While most households (64\%) have fenced off their pastures, other households (36\%) continue to cooperate on a small scale with neighbouring households who are usually families. The cooperation includes sharing pastures, infrastructure (e.g. wells) and machinery, as well as labour cooperation. Larger scale and lawful cooperation is considered to be a critical opportunity for the sustainability of the grassland and the well-being of herders by both insider herders and outsider experts (e.g. Da, 2008; Wang, 2009).

${ }^{48}$ Ao is a village leader I interviewed. 


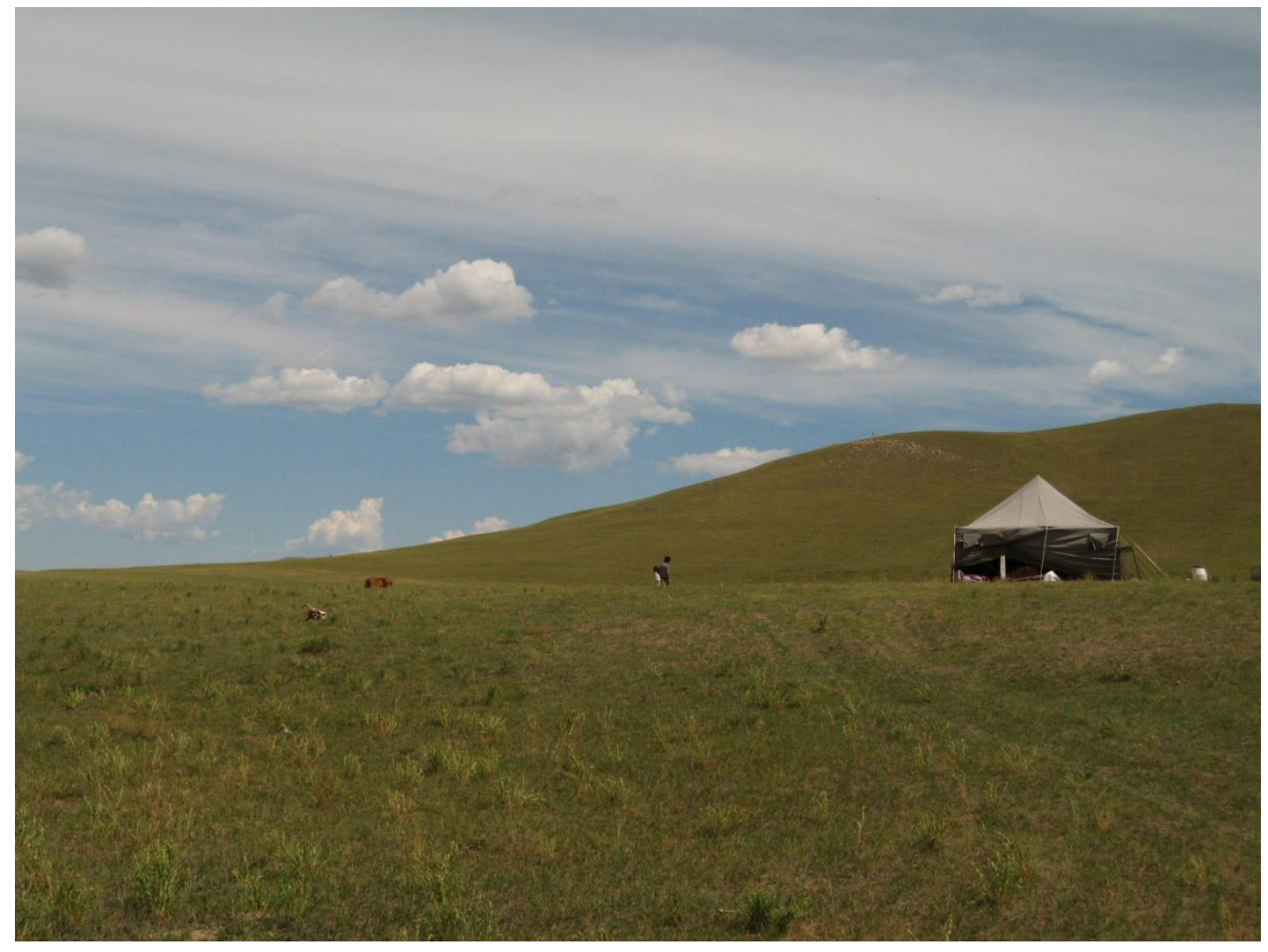

Figure 5.1 The pasture in Bayin gacha (photo by: Ruifei Tang, 2010).

In addition, I also interviewed two leaders of Hulun Be'er Pastoral Cooperative ${ }^{49}$ in Hulun Be'er gacha ${ }^{50}$ (located at the north-east end of Inner Mongolia) through telephone communication. The contact with this cooperative was provided by the leaders of Aolin cooperative. The reason for carrying out this additional data collection was that Hulun cooperative had undertaken cooperative activities since 2008, and achieved village-wide herding rotation and revitalised traditional institutions at certain degrees. Their practices provide valuable information regarding my research target.

Hulun gacha shares several common attributes with Bayin gacha, including population structure, economic status, institutional change, grassland degradation and the development of a cooperative organisation. The territory of the village is 39,000 ha, comprised of 44 households (Mongolians accounted for $98 \%$ of the total population). Within these families, about half of them possessed livestock while the other half only possessed the land without any livestock. The total number of livestock was about 7,300

\footnotetext{
49 It will be abbreviated as Hulun cooperative below.

50 It will be abbreviated as Hulun gacha below.
} 
in 2010, including 7,000 sheep, 200 cows, and 100 horses. Land and livestock privatisation was enforced between 1993 and 1996. The perceived pasture degradation was reported since 1996, and was considered a result of sedentary herding activities by the interviewees. For the families with livestock, due to the grassland degradation, increasing cost of hay harvest and purchasing animal fodder for winter use, herder's lives had become more and more difficult. They often struggled with the difficulties of working at individual family level without any support or collaboration. For these livestock-less families, many of them rented out their land to outsiders who were able to afford livestock raising. The land tenants generally showed very limited concern for grassland protection.

Note: Please see Chapter Two for the details of research methods.

\section{RESULTS}

Based on the findings from multiple research methods (i.e. semi-structured interview, focus group discussion, participant observation and literature review), this research explored four major categories as results. In this section, I will first illustrate the historical governmental policies and their impacts on community institutions and traditional practices over the last 50 years; then explain how the Cooperative Law has been implemented at the local level, how the community cooperative was formed and how the cooperative operates at the research site. Finally, I will illustrate the benefits and difficulties of the cooperative as perceived by the informants.

Please note that, there are four different terms used to distinguish the information sources in this section: 1) an interviewee is always further defined as community interviewee, expert interviewee or official interviewee; 2) a focus group discussion informant refers to a community participant of a focus group discussion; 3) community informants indicate a mixture of community interviewees and focus group discussion participants; and 4) research informants or all informants refer to a wider mixture of interviewees from different groups, and community focus group discussion participants. 


\subsection{The governmental policies and their impacts on community institutions and traditional practices}

In Inner Mongolia, ever since modern China was established in 1911, there has been a limited level of local Mongolian autonomy (Ao, 2003; Williams, 2000). The current decision-making system has been constructed and run by the centralised government. Since the 1950s, the central government has continuously endeavoured to "uplift" indigenous Mongolians from traditional nomadic pastoralism towards a modern, sedentary regime (Ao, 2005; Da, 2008). This research focuses on the change of the government's policies and the corresponding change of community institutions and traditional practices over the last 50 years. There are two reasons for scoping the research with this time frame. First, the last 50 years are recognised as the most significantly changing period in the research area by both the literature (e.g. Da, 2008; Da and Zheng, 2010; Wang, 2009; Williams, 2002) and research informants (e.g. Ao, Ba, Aude, interviews, 2010). Second, the age structure of the community informants $(\leq 34 y r s, n=33 ; 35-54 y r s, n=84 ; \geq 55 y r s, n=15)$ determined the earliest date they can recall from their life experiences is the 1960s. According to the significance of policy and institutional changes, three distinct periods are identified: 1 ) the grand commune era in the 1960s and 1970s; 2) the privatisation era in the 1980s and 1990s; and 3) the national western development era from 2000.

\subsubsection{The 1960-1970s, the Grand Commune Era}

In the early 1960s, the Grand Commune Policy was enforced by the central government. The movement was a resource and property nationalisation process. Despite the previous land and property ownership, central government claimed all land and resources as state property (Ao, 2003; Da and Si, 2006). In Inner Mongolia, the traditional communal ownership of the land and resources was eliminated. Traditional social organisations, such as elder leadership and traditional working units broke down. All herders had to join a commune and work within the commune territory under the leadership appointed by the government.

As all elder community informants (aged over 55, $\mathrm{n}=15$ ) and most middle aged community informants (aged between 35 and $54, \mathrm{n}=56$ ) recalled, the grand commune 
was introduced into the region in the early 1960s. All land and other resources were claimed as assets of the commune, and all herders were assigned jobs by the government-appointed leaders of the commune. The commune leaders were sometimes Han Chinese ${ }^{51}$ from outside of the community. The commune applied a working credit system, in which labour was converted into credits. For example, an adult male could earn five credits by a full day of work. By the end of a month, the commune committee would convert these credits into cash and disperse it to the community members as salary. A well-experienced herder could earn NZ\$80 (equivalent in 2012 dollars) per month.

As many community informants $(n=79)$ and all expert interviewees $(n=11)$ claimed, this resource-nationalisation policy and its follow-up practices ended traditional resource management and traditional institutions. First, the establishment of the communes ended the Haote system and traditional elder's leadership, which lasted for thousands of years prior to the 1960 s. Haote, literally meaning a place inhabited with people, was the name of a basic production unit. It was often comprised of three to five, sometimes up to eight to ten households. The households within a Haote were usually blood-related. Traditionally, the day-to-day decisions were mainly made by the Haote leaders, usually the elders. For major issues, such as seasonal rotation plans, Haote would hold public meetings, in which every member could have their say. The elders usually had the final words on these decisions. As the oldest person in the village recalled, "Elders were [the] most experienced herders. Their knowledge and experience earned their reputation and leading positions in a community. All young members fully respected and were willing to follow elders' judgements and decisions. Everyone was happy and cooperated, and there was hardly any argument or conflict" 52 . Another nine community elder interviewees and 23 middle-aged community interviewees affirmed this statement.

In the grand commune era, instead of the traditional elder's leadership, the commune leaders were responsible for making decisions regarding production and other issues. Community members, on the other hand, did not have any participation in either choosing the commune leaders, or making decisions about resource use. For example,

\footnotetext{
51 The ethnic majority in China which counted for about 1.18 billion in 2010.

${ }^{52}$ It is worth noting that, when an informant recalled the past, there was a potential that some of their opinions might be overly romantic. It may potentially generate nostalgic bias to the research results.
} 
as one focus group informant noted, "In 1966, after the commune formed, two Mongolian herders who had completed primary school (the majority of the herders were illiterate at that time) and a Han Chinese from Gansu Province (a neighbouring province) were appointed as the commune leaders by the officials. But they were not as knowledgeable as our elders, especially the Han leader, who had no previous experience in herding".

Second, the commune system also ended the traditional communal ownership and patterns of pasture use. Traditionally, herders were free to move according to their own preference, and rotated without restraints or strict boundary limits. The rotation range was determined by their travelling capacity and the need for rotation. Mongolian herders considered the grassland to be a common resource, which they shared and cared for equally (Da, 2008; Tang and Gavin, 2010). Everyone who lived on the grassland was responsible for protecting and sustaining it, as grassland is the collective source of their livelihood. Since the grand commune movement, the originally undivided, open-toshare grassland had been redefined with boundaries. In the replacement of Haote, the basic production unit became fenchang (literally means sub-pasture). Differing from the natural kinship basis of the Haote system, fenchang was an artificial geographical division created by the local authority. A fenchang usually contained more than 100 households; several fenchang formed a commune, which usually defined boundaries from other communes. Households who belonged to a commune were required to live within the commune's territory and worked collectively. As one community elder interviewee noted, "In 1965, a fenchang formed in this place. Its territory was similar to our village nowadays. An official came to persuade us to join the fenchang. He said, everyone in the fenchang would equally own the pasture and livestock, and work together would make us stronger. We and another ten Haote joined the fenchang. And since then, we have lived around the fenchang until now".

Despite the changes mentioned above, the herding practice remained nomadic. The livestock herds were still able to travel freely from point to point, as long as within the commune's territory. As one elder interviewee noted, "I was selected as a commune herder in 1963. In the winter of 1967, our herd moved 12 times within three months. Every 10-15 days, we moved the herd from one pasture to another. With a tent on the 
ox-cart, we herded sheep and cows and travelled 10-15km a day. We grazed the herd along the way. We started the trip at the beginning of December, and came back in the end of February in 1968". The eldest community member summarised Mongolian herders' lives during the grand commune era as, “... the grassland remained in a relatively pristine condition, the climate pattern was regular, but herders' lives were harsh and very poor. The salary I earned could only sustain a very basic level of living, and everyone in the commune was the same".

In conclusion, the most significant change in the grand commune era was the breakdown of the Haote system and traditional elders' leadership. Another fundamental change underlying these was the change of ownership of the land and resources, from traditionally collectively owned to state-owned.

\subsubsection{The 1980s-1990s, Settlement and Privatisation Era}

The voluntary settlement policy was implemented nationwide since the 1970s in China (e.g. Inner Mongolia, Xinjiang, Tibet, Yunnan, Sichuan and Guizhou). The policy aimed to transform nomadic herders to sedentary residents. In the research site, most of the community informants $(n=98)$ remembered clearly that the settlement policy had been implemented in the late 1970s. The local government established a medical centre, veterinarian service and a primary school in the nearby sumu (township). The government intended to attract herders to give up nomadic herding rotations and stay around the sumu all year long by providing free medical and veterinarian services only in the sumu. However, most of the herder families preferred the traditional nomadic lifestyle.

The launch of the Economic Opening Up Policy in 1978 indicated the commencement of the Chinese economic reform. The central theme of the reform was applying market principles to trigger national economic development. The reform consisted of two stages: 1) from the late 1970s to the early 1980s, involving the de-collectivisation of the agricultural sector, permitting the formation of entrepreneurs, and opening the country to foreign investment; and 2) from the late 1980s to the 1990s, involving the privatisation and contracting out of previously state-owned industry and rural lands, 
and relieving the central price control system (Zhao, 2008).

As a national priority in the 1980s, privatisation was commonly believed to be the miracle trigger for economic development in China (Alcorn and Toledo, 2000; Li et al., 2007; Williams, 2002). It is worth mentioning that within this process the land remained state-owned; only the land user right was leased to individual households for 30 to 90 years (Ao, 2003; Cao et al., 2011). In Inner Mongolia, the household responsibility system replaced the grand commune. Herder families were granted a certain number of livestock and user rights for a pasture. In the 1990s, it was common that herders had to settle down on their "own" lands, and rotations to or through others' land was not possible anymore. Gradually, more residential houses were built instead of traditional yurts. This was a crucial era that changed Mongolian TEK and cultural traditions completely - the thousand-years-long, traditional nomadism was terminated. Community members increasingly worked individually (based on family units), and their traditional collaborative institutions were continuously diluted (Wang, 2009).

In Bayin gacha, as some community informants noted, the commune started to distribute all collective livestock to individual households based on the size of household (roughly about 50 livestock per person) in 1983. In the following years, pastures were allocated to each household according to their livestock shares. The allocation of livestock and pastures was considered fairly equal by the community informants. Since then, the state government recognised herders' ownership of livestock, and their exclusive user rights of the land and pasture they lived upon. The privatisation processes were finalised in the 1990s when the majority of the households fenced off their lands. The use of division fences was encouraged by the local authority ${ }^{53}$. While a considerable number of families (about 64\%) fenced off their pastures under the encouragement of government policy, some families remained open-pasture and shared it with their neighbours, who were usually blood-relatives. The completion of the privatisation processes indicated the end of Mongolian traditional nomadism. Since then, all families in Bayin gacha have settled down and lived in their fixed locations. All the community elders interviewees $(n=15)$ claimed they perceived grassland

\footnotetext{
53 The government provided a subsidy for the families who fenced off their pastures. The average level of subsidy was about NZ\$1/metre, while the total cost of the fence was NZ $\$ 2 /$ metre.
} 
degradation starting in the 1980s, while other informants generally considered the 1990s to be the beginning of land degradation. This difference of perceptions could mainly be caused by different life experience, in which the elders have had a longer time frame to observe and compare. All the community and expert informants $(n=143)$ strongly linked the grassland degradation with a sedentary lifestyle. All the community informants $(n=132)$ emphasised that without proper rotation, the pasture has been over-used and had no chance to recover from grazing. As one community interviewee described, "Our traditional nomadic lifestyle is not just a cultural preference; it implies an important principle of living in grassland - never over-use a patch of the grassland. Rotation enables pastures to recover from grazing. Usually, if it rained, a used patch of pasture could quickly recover and get ready for the next turn of grazing".

Some informants also recognised climate change, especially the frequent droughts, as a major cause of grassland degradation. A focus group informant recalled, "There was plenty of rain and snow before the 1990s, and we didn't need to worry about the regeneration of grass. But since the late 1990s, droughts have happened more and more often. The grassland has degraded very obviously. Meanwhile, herders cannot rotate their livestock, and animals have to constantly graze on the same patch of pasture. It leaves no time for [the] grass to regenerate".

To cope with the limits of sedentary production, herders had to start to preserve hay for winter use since the 1990s. Without the possibilities of travelling southward for winter grazing, as in the nomadic tradition, every household had to fence off a grazing-free zone as a hay reserve. The hay would be harvested in early autumn, and stored away until its winter use. The size of a hay reserve could vary from 33 ha to 66ha, which depends on the total area of a household pasture and the condition of the grass. In most of the cases, the hay couldn't sustain the herd through a winter. Herders had to buy extra fodder (e.g. dried corn) from the market. The hay harvest and storage, and the purchase of extra fodder hugely increased the cost of production. For instance, large machinery is an essential requirement for hay harvest. For most families, the hay harvester might be affordable (NZ\$1,500-2,000), but the haymaker (NZ\$15,000-20,000) was usually beyond their financial capability. Haymaker renting would cost them NZ\$500-800 for 
two or three days use at the hay harvest season ${ }^{54}$. All these new costs of the sedentary lifestyle significantly increased the pressure and difficulty to Mongolian herders when compared to their traditional nomadic lifestyle.

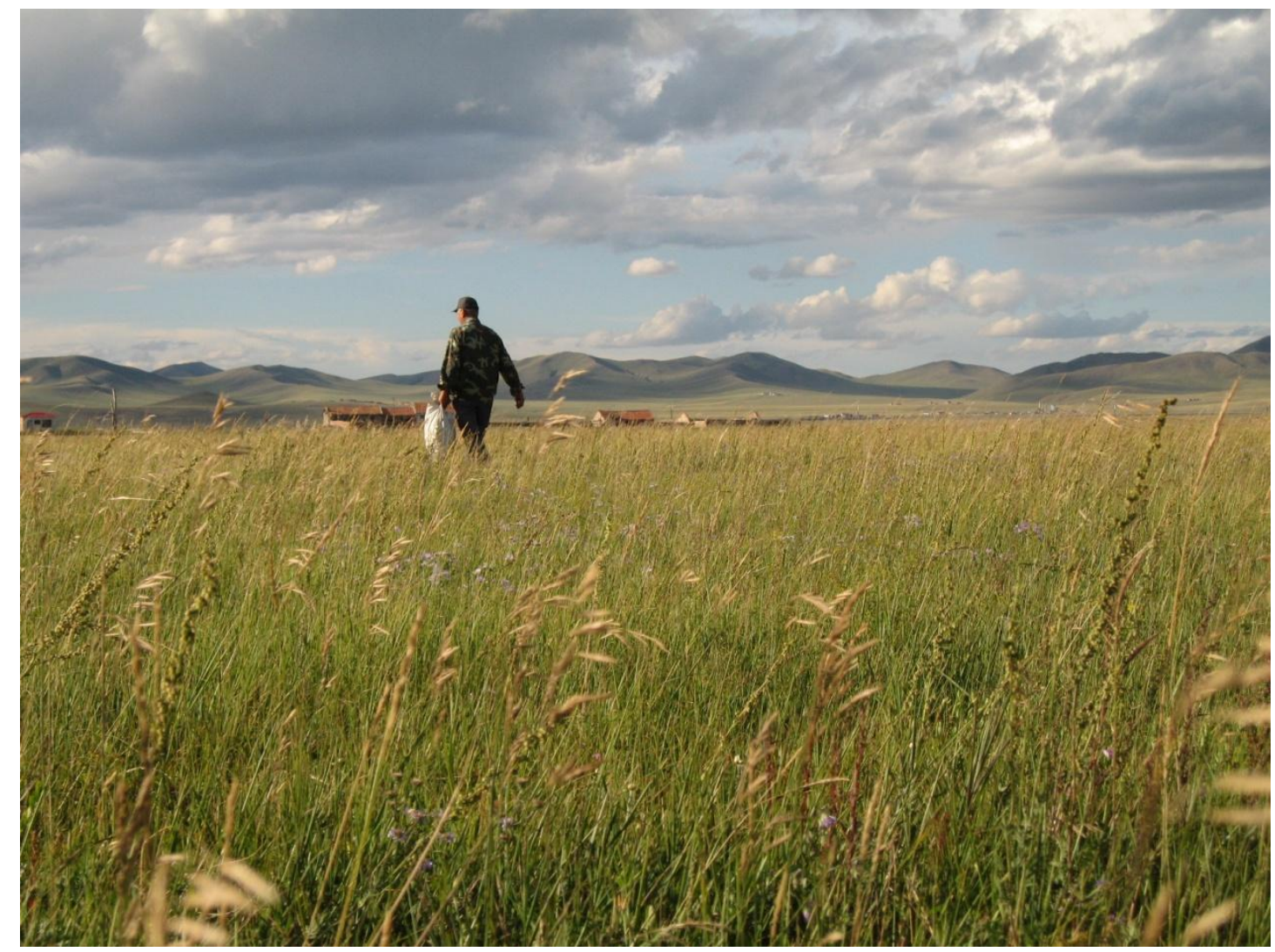

Figure 5.2 A family hay reserve in Bayin gacha (Photo by: Ruifei Tang, 2010).

As many community and expert informants $(\mathrm{n}=89)$ emphasised, the loss of traditional natural disaster relief mechanisms is another significant change to traditional institutions and practices in this era. In the past, herders collectively coped with natural hazards or disasters (e.g. droughts and snowstorms). For instance, if one Haote encountered a drought, the families would travel to another area which had better weather. The hosting Haote there would be happy to accept their visit and agree to their herding activities in the area; as return, the hosting Haote could receive the same aid from the visiting Haote when needed. As an elder interviewee claimed, "We all shared the grassland. If someone had to travel to our pasture to avoid a drought or snowstorm, we were happy to share. This was because we knew that we could travel to their place for aid when we had problems in the future".

54 The average annual income was about NZ\$4,000-5,000/family. 
This was a very equal and mutually beneficial relationship that was traditionally carried out on the grassland. In this way, herders shared their risk and cost of natural disasters. But since the privatisation, individual families are tied to their contracted lands and work on their own; they do not have any alternative site to go when there is a natural disaster. The cost of the disaster has to be paid by individual families. The only hope for external aid is from the government. Nonetheless, the government only grants aid when there is an extreme weather event. For example, in 2005, a snow storm hit most areas of Inner Mongolia. It was recorded as the largest storm over the last 61 years (Ao, interview, 2010), and led about 20,000 households into poverty due to the complete or partial loss of livestock. Though the government delivered disaster aid, such as foods and clothes to mitigate the immediate threats to herders' lives, for their long-term wellbeing after the disaster, the government did not show much consideration and action. Apart from governmental support, there was no alternative aid provided to the disaster affected families (Hote, interview, 2010).

\subsubsection{0 onward, the Western Development \& Conservation Era}

Since 2000, a range of economic development-oriented policies have been carried out in the background of China's Western Development Strategy (the Strategy), in which 12 western provinces and prefectures are included (e.g. Inner Mongolia, Tibet and Xinjiang). The main outcomes of the Strategy include the development of infrastructure (e.g. transport, hydropower plants, energy and telecommunications), the promotion of modern education and the attraction to foreign investment (Hu, 2005). Increased ecological protection is also a part of the Strategy. The Strategy guarantees that these 12 western provinces will receive national funding for development. For example, between 2000 and 2005, a total of NZ\$92billion was spent on building infrastructure in western China $(\mathrm{Hu}, 2005)$. In response to the Strategy, the regional and local level governments in Inner Mongolia have produced and enforced a series of measures to implement the Strategy. Industry was favoured over pastoralism. As a key component of local GDP, mining (mainly including coal, rare earth metals, mirabilitum and alkali) has increased dramatically since 2000. Based on profits from mining, Inner Mongolia had the fastest GDP growth in China between 2002 and 2009, and reached NZ\$194.5 billion in 2009 (The Bureau of Statistics China, 2010). 
However, in the meantime, grassland degradation was reported widely (e.g. Su et al., 2004; Tong et al., 2004; Wang, 2009; Williams, 1996). According to Wang (2010), there were 63.59 million ha of productive pasture remaining in Inner Mongolia in 2009, which accounted for about $60 \%$ of the pasture area that was presented in the $1960 \mathrm{~s}$. The government (e.g. APFB, 2002, 2006) and some literature (e.g. Su et al., 2004; Yan et al., 2005) recognise that over-grazing is the main driving force of the degradation. For example, the Inner Mongolia Bureau of Environmental Protection (2006) stated in its Annual Report 2005, "the large scale desertification occurring in Inner Mongolia is caused by human activities, including over-grazing, over-reclamation, over-harvesting of firewood and unsustainable use of groundwater". Accordingly, the government grassland conservation measures focus on setting up fenced reserves, reducing livestock numbers and removing herders to urban areas (Cao et al., 2011). Disagreeing with this "mainstream opinion", some scholars argue that the sedentary practices and privatisation are largely responsible for the land degradation in Inner Mongolia (e.g. Cao et al., 2011; Tang and Gavin, 2010; Wang, 2009; Williams, 1996). However, to date, there is no official report or study of the effects of mining and other development activities, such as railway and road construction, on grassland ecosystems and local livelihoods in Inner Mongolia.

Most of the community informants pointed out that the grassland ecology and their lives have changed most significantly since the 2000s. Herding activities have been disregarded by the local government; instead, industrialisation and urbanisation have been its major pursuits.

\section{Industrialisation}

Some expert interviewees and official interviewees pointed out that, as the response to the Western Development Strategy, the local government has emphasised GDP growth and industrialisation in the region since 2000. According to these interviewees, the importance or development of pastoralism has not been mentioned in either governmental documents, or local officials' public speeches over the last ten years. For example, the local government of the Baiyinhua Town (current population of 8,000 people) has set up a development plan to transform the town from a pastoral town to an 
energy production capital with a target population of 300,000 in 20 years (Ba, expert interviewee, 2010). The town's main industry will be coal mining and power plants, which will aim to provide power for big cities in north-eastern China. The local government attracts herders to work in the factories by promoting factory jobs, and providing low-price-apartments in the township. There are more than a thousand mines in Xiwu Qi, including coal, iron, zinc and other metal mines (Da, expert interviewee, 2010). The owners/developers of mines are mostly from outside of the region. Many community informants perceived that these mine owners and workers come only for the mining and, in turn, did not care about the health of the local land at all.

All informants, apart from local official interviewees, agreed that mining has been the most destructive factor to pastoral practices and local lifestyles in the research area. As one focus group informant stated, “There was no chance to say 'No' or negotiate with miners, because the officials and government are supporting them. If a mine is set up on your land, with very little compensation you have to move out. If a mine is set up in your neighbour's land, you will suffer from the open mining pollution: dusty wind, poisonous water discharge and endless noise".

On the contrary, the government officials I interviewed considered the mining activities to be uplifting to the local economy and contributing to overall local development. As an official interviewee in Xiwu Qi claimed, "The mining industry has brought significant economic development to our region. It generates jobs and contributes to local GDP growth. This is why the government is able to build a number of major infrastructure in Xiwu Qi over the last five years, such as a town civic square, a public park, a new hospital and more than ten new residential blocks. These are all beneficial for herders' wellbeing". However, the benefits this official mentioned above were provided for the urban population in the regional capital city - Xiwu (about 40,000 people, including Han and Hui people ${ }^{55}$ ), but did not provide much benefit for the herders who lived in the pastoral areas (about 80, 000 people) (Da, expert interviewee, 2010; multiple focus discussion informants, 2010).

\footnotetext{
${ }^{55}$ An ethnic minority group in China with a population of 10,590,000 in 2010. Mongolian population in China was about 4,500,000 in 2010.
} 
There are a number of adverse effects of mining activities towards the local community's wellbeing that were mentioned by the community and expert informants. The most direct effect to herder's life is the occupation of the land for mine development. In the Xiwu Qi region, many herders were removed and relocated from mining sites. Most of them moved to Xiwu, and struggled to make a living there. As a Mongolian expert interviewee who lived in Xiwu described, "There are many issues associated with mining immigrants. Those herders don't have particular skills for urban living. Some of them hardly speak any Mandarin, which is necessary for finding an urban job, such as a shop attendant or a factory worker. Many of them spent the relocation compensation money within a few years, and then lived in absolute poverty. Street beggars started to appear in Xiwu within the last few years. This has never happened to our people before, because basic life needs were easily satisfied by herding lifestyle. Our livestock provided all our basic needs".

In Bayin gacha, 16 households have been displaced by mining activities since 2000 . Despite the various compensation packages that mining companies offered to herders, the local government paid NZ\$210 per hectare as compensation to the mining immigrants, but there is not any supporting plan for their on-going livelihood. As a focus group informant stated, "Without informing us, a mining company came and dug a hole (500m in diameter) for mining exploration on our pasture in 2008. They claimed that they found zinc in here. Then the village leader came to tell us we had to sell the land to the mining company, because mining is government priority. We didn't have any other choice, but sold most of our pasture to the mining company at a very low price and moved to Xiwu. However, we were able to keep a small patch of pasture for our yurt. We don't want to cut off our connection with the grassland. Once in a while, we come back from Xiwu to stay in our yurt".

Herder families who have not been relocated have to face a number of serious challenges as results of the mining. In order to minimise the cost, most of the mines are open mines that generates a large amount of dust pollution in the area. An active mine constantly produces dust and sand which is carried by wind and smothers the ground vegetation in the surrounding areas (Li, expert interviewee, 2010). Most of the community informants complained about the grass degradation caused by the dust from 
the mines. Another major issue of mining is its over-use of ground water, which leads to ground vegetation deterioration. According to an expert interviewee, an average-sized coalmine consumes about $1000 \mathrm{~m}^{3}$ of ground water per year. An elder interviewee claimed, "In such a climate sensitive area, ground water is the most crucial natural resource that determines our livelihood. Since 2000, grassland has been continuously degraded from drought and mining. Now, even if it rains, the soil is not capable to store water, because the vegetation cover has gone". When mining competes for ground water with the local population's living and production requirements, it also discharges polluted water onto the land. Livestock deaths have been attributed to drinking contaminated water. The discharge is also harmful to the ground vegetation ${ }^{56}$.

\section{Urbanisation}

As another critical component of local development, urbanisation has been a priority of the local government in the 2000s. As many informants recalled, in 2002, the local government applied a policy called eliminating townships and combining towns. By implementing this policy, more than half of the townships in pastoral areas were eliminated. All social services (e.g. medical centres, veterinarian service and primary schools) which used to be located in each township are now gone. All school-aged children have to attend schools in Xiwu. While the school children stay in the city, one or more adults from the family have to stay in the city to look after them. This increases the expense of education and leads to a population flow into the city. As one community interviewee explained, "There used to be a school in the sumu (township), about 40 minutes of horse ride (roughly equal to $20 \mathrm{~km}$ ). It was a boarding school, in which all children from our village went to. The children usually came home every weekend. Parents sometimes visited their children during the weekdays. But now, all our children have to go to the city for their education. They only see their parents in the school holidays, twice a year".

According to my observation (2010), the daughter of my host family (an 11-year old girl) had to travel six hours with her mother to Xiwu for a new school term. During the school term, she had to stay in the city and live with her grandparents (who moved to Xiwu to

${ }^{56}$ These are perceptions noted by interviewees, focus group informants and my observation. Due to the political sensitivity of minority issues, the publication regarding mining issues in Inner Mongolia has been suppressed in China, in both academic sources and public media. 
look after her) in a rented apartment. Her parents supported the grandparents for the expenses of living in Xiwu.

The government also strongly encourages herders to move from the pastoral areas to the city and towns (Bu, expert interviewee, 2010). For example, in Baiyinhua Town, the government has developed 15-20 blocks of apartment compounds, and exclusively offered a low price to herder families including several loan options. The target group of this urban immigration plan is the herder families severely affected by mining activities, who lost some or all of their land for herding (Bu, expert interviewee, 2010). The government also promotes jobs for the energy plant to attract herders to move into the city and towns.

\subsection{The Cooperative Law implementation at the local level}

After the enactment of the Cooperative Law in October 2007 (which aims to narrow down urban-rural income disparity and to achieve a certain level of economic and social equity in China), the local government has applied the policies and measures that the Cooperative Law requires, including registration aid, tax relief and other policy support (Du, official interviewee, 2010). For instance, in terms of registration aid, the local Industry and Commerce Bureau set up a Mongolian language service. The service includes translating all registration materials into Mongolian, and arranging Mongolian speaking officials to serve Mongolian herders. The registration process is shortened to one to three working days when compared with usual corporate registration (ten working days), and is free of charge. Other policy support can vary from time to time. For example, in 2009 the local government published a policy that cooperatives which contain more than ten households are entitled to apply for loans from the local Rural Credit Unions.

The registration of cooperatives in Xiwu Qi started in early 2008. According to the Xiwu Qi Industry and Commerce Bureau (Zhang, official interviewee, 2010; Guo, official interviewee, 2010), 29 cooperative organisations have registered since then. The majority of these cooperatives (90\%) concentrate on pastoral production with a focus on revitalising traditional herding practices, while two of them are based in town areas 
and focus on information and technical services to pastoral production.

While all the official interviewees had positive opinions on the implementation of the Law and its supporting policies, some expert interviewees criticised that these policies and measures have not reached ground level. For instance, the policy "to encourage the development of herder's cooperative organisations" was mentioned for the first time on the Xiwu Qi local People's Congress, which is the top level of the local authority. However, there was no further implementation plan attached. Whilst satisfied with the registration process and tax deductions, the cooperative members expressed their expectation of further and more substantial support from the government, such as financial subsidies or loans for traditional herding activities.

\subsection{How do cooperative organisations work?}

\subsubsection{How was Aolin cooperative formed?}

Aolin Baolige Cooperative Organisation (Aolin cooperative) in Bayin gacha was formed and registered with the local Industry and Commerce Administration Bureau in 2009. The registration was free and took only one day to be processed. The cooperative was initiated by the current village president, who had been well informed about the Cooperative Law and relevant governmental policies. In early 2009, he persuaded his own family and another five families (including three of his relatives' families and two neighbouring families) to initiate the cooperative. Later, he invited a respected elder in the village to join the cooperative and to contribute his suggestions and ideas. The respect for, and the belief in elders' knowledge and leadership was frequently expressed by community informants. As one community interviewee stated, "We were hesitating to join, because we were not sure about the outcome of the cooperative according only to the president's word. But when we heard Aotegen (a respected elder) had joined, we thought we'd like to follow him, because we trust his judgement". Since the elder joined, a considerable number of families $(n=6)$ also joined the cooperative. By 2010, 12 households had joined the cooperative.

All the informants that were members of cooperatives stated $(n=20)$ they were willing to cooperate and happy to take collaborative actions. Everyone was actively involved in 
discussions and meetings, and agreements came easily. As an elder noted, it was similar to the traditional Haote decision making process. For example, at several meetings of the cooperatives, in which all members participated, decisions regarding job division, pasture use pattern, and the rotation cycle were made collectively. The cooperative members also elected their organisational leaders. The current village president and an elder earned their positions in leading Aolin cooperative.

A local non-governmental organisation (NGO) called Hari Gentai Green Animal Byproducts Development Association (Hari association) assisted the formation of the cooperative. The NGO was formed in 2005 with the aim of providing information services to rural organisations. The organisation was based in Xiwu city and had resources to access updated governmental policies and NGO networks around China. It was able to help the cooperative to connect with other cooperatives and NGOs, as well as to provide funding information. In return, the $\mathrm{NGO}$ was granted funding by other organisations (including an international foundation) in recognition of its efforts to assist community level conservation and development projects.

\subsubsection{How does Aolin cooperative operate?}

The primary focus of the cooperative was to form a collective working group and revitalise herding rotation practices. The initial participating households $(n=6)$ removed the fences around their properties and merged the pastures and herds. The joint pasture accounted for 1,333 ha. After an analysis of vegetation condition and topography types, the cooperative divided the joint pasture into two functional regions - 333 ha as a hay reserve, and 1,000 ha as herding pastures. The herding pastures were further divided into three sections, each contained multiple sub-sections. The joint herd started grazing from one sub-section, and rotated to the next when it was needed. The rotation among sub-sections was very frequent, sometimes on a daily basis. The rotation among the three main sections was on a monthly basis. However, all these rotations were flexible as the joint pasture was not big enough to sustain a seasonal rotation pattern.

The most experienced herder and his family were selected to be the chief shepherd. With the assistance of another two families, the chief shepherd's family decided where 
to graze, when and where to rotate at a daily basis. Another three families carried out jobs like hay harvest, livestock nursery, and collecting firewood and cow manure for heating purposes. The cooperative members treated job division liberally, swapping roles from time to time. For example, in the hay harvest season (usually 7-10 days), most of the cooperative members worked together in the hay reserve and left one or two people to graze the herd. Likewise, in spring, most of the members were occupied with the lamb and calf nursery.

Hay harvest is another major task requiring collective efforts. As a focus group informant stated, "Ever since the 1980s, hay harvest has become extremely vital to us. It determines the survival of livestock over the winter, and consequently the annual income in the following year". Nevertheless, hay harvest and storage has been a major obstacle for individual households to deal with. For instance, there were only two families in the village rich enough to own a haymaker (cost about NZ\$15,000-20,000), and this left no guarantee that all the other families would have a chance to hire it in time. The risk of delayed renting was that rain could ruin the harvested hay waiting in the open field. A community interviewee recalled, "Three years ago, we couldn't find a haymaker to hire. Even though all of our relatives and friends came to help us to pack the hay, we could only do it very slowly by hand. Then it rained for two days... About half of our hay was mildewed. In that winter we had to buy fodder from a trader to feed our livestock, and that cost us about 14,000 Yuan (NZ\$2800) in cash. It was almost half of our total (gross) income that year".

The huge amount of labour required and the significant cost of buying, maintaining and/or hiring machinery are difficult for individual families to deal with. Members within the cooperative now work together on hay harvesting. They share the machinery provided voluntarily by the village leader. As he expressed, "I offered our haymaker for the cooperative to use for free. All participant families worked together in the hay harvest season, and they didn't worry about the difficulty and cost of hiring a haymaker. I just want to prove that the cooperative is a good solution for our current difficulties, and attract more families to join". 
In the future, the cooperative expects all families in Bayin gacha to join the organisation. This will enable four-season herding rotation to be revitalised throughout the gacha. Meanwhile, the cooperative is also considering the possibility of organising sheep trading activities collectively. At present there is no trading organisation for herders, whose annual income generated from selling sheep, cows and wool relies heavily on mobile traders. This is because of the long distance to the market and the unfamiliarity with the outside market. Meanwhile, an individual herder family is not able to afford large vehicles to carry products directly to outside markets where they could obtain a better price. The income of herders critically depends on how much the mobile traders offer. As a result, herders are in a disempowered position in this trading process.

To strengthen market competitiveness and explore better market options is another target of the cooperative for the near future, as noted by a cooperative leader. With the expectation of more families joining, the cooperative plans to organise its own marketing and trading activities. A cooperative leader explained, "In an ideal scenario, the cooperative will become a market-recognised product provider. This will not only increase the income of herders, but also reduce the risk of dealing with mobile traders".

\subsubsection{A comparable cooperative from Hu Meng}

Hulun Be'er Pastoral Cooperative (Hulun cooperative) in Hulun Be'er gacha (Hulun gacha) of Hu Meng (located in north-east Inner Mongolia) was established in 2009. Similar to Aolin cooperative, Hulun cooperative was initiated by the existing village leaders. The two village leaders targeted several respected elders in the village and consulted with them about how to carry out herding rotation and how to divide the pasture into seasonal use. They also visited and discussed their ideas with several grassland ecologists and Mongolian culture experts, and obtained their advice on cooperative development. In 2009 at a village meeting, the village leaders explained the idea of forming a village-wide cooperative and all 44 households joined the cooperative voluntarily. The local government actively supported the development of the cooperative by contributing an unconditional grant $(\mathrm{NZ} \$ 120,000)$ to the cooperative in 2009. The cooperative was then able to buy a hay harvester and a combined agricultural truck, and to finance their joint-pasture project. 


\subsubsection{How does Hulun cooperative operate?}

The main actions of the cooperative included de-fencing the pasture, combining pasture and livestock herds, and allocating labour into four working units. Apart from a small amount of pasture which was leased out until 2012, the village-wide, joint pasture was divided into five parts - four parts for seasonal rotations (spring, summer, autumn and winter pasture), and one part for a hay reserve. However, the current rotation was restrained by the gacha territory $(39,000 \mathrm{ha})$, and the division of a hay reserve (about 7,000 ha). The effective area of the current four-season rotation was about 32,000 ha (across over $25 \mathrm{~km}$ ) in 2010. This was smaller than traditional nomadic rotation area (across over $100-150 \mathrm{~km}$ a year).

The elders actively contributed their knowledge of land division and land use during the decision-making process, and all herders usually agreed on their advice. For example, the summer pasture, the most important resource for sheep to attain an ideal weight was located near a lake, where sheep had access to a water source and short grass. According to the elders, the short grass is nutrient-rich, and the better visibility it provides can also protect livestock from wolf attacks. In 2010, the village did not lose any sheep due to wolf attacks. A loss of 10-20 sheep was usually recorded prior to the cooperative.

All the participating families were divided into four working units - a herding unit, a fodder unit, a marketing unit, and a tourism unit. All villagers participated in the decision making of job divisions. The members of different units were either nominated or self-nominated. For the herding unit, nine families were selected because of their rich experience in herding. The cooperative's livestock was divided into nine herds with 1000-2000 sheep in each. The herding unit families decide about where to herd, and the rotation cycles and routes. For the fodder unit, three families were selected to be in charge. Their main jobs included hay harvest, storage and the delivery of hay to the herds in winter and early spring. During the hay harvest season (August to September), each family contributed one or two people to help the fodder unit. The hay harvesters and haymakers were used within the cooperative. In 2010, the fodder unit was able to supply the herds with sufficient feed, thereby avoiding the extra cost of buying additional fodder. 
Five younger families were selected for the marketing unit. Their main jobs were exploring pastoral markets for their products - sheep meat, wool, and occasionally surplus hay. The development of the marketing unit is on-going. The tourism unit was comprised of four families. Hu Meng is a summer holiday destination for the nearby urban population. In the past, tourism was run by companies from outside the gacha. Now the cooperative has taken the lead of tourism development in their community. Families who worked in the tourism unit created yurt home-stays for tourists, which attracted a significant number of tourists from both local (e.g. nearby townships) and non-local areas (e.g. Beijing).

\section{$\underline{\text { 3.3.3.2 Benefit sharing and profit allocation }}$}

In 2010, the cooperative shared the first profits of their herding production among the livestock owner participants. The allocation mechanism was recognised as an informal joint-stock system, which combined a traditional norm of equal shares with a modern shareholding regime. The basic idea was that the number of livestock contributed by a participating family determined their share of the profit. Likewise, the herding-related cash investment quantity was also determined by the number of livestock. On the other hand, for the families without livestock, the land areas that they contributed into the cooperative determined their share. In addition, all the participants agreed that the cooperative was responsible for helping the poorer families in the community, and they were all willing to share their benefits with the families who needed financial aid. For example, there were three families without livestock and had a small area of land, and were considered the poorest in the community. The cooperative selected them for the tourism unit. Without immediate benefits from tourism, the cooperative paid these families a monthly salary of NZ\$400, which came mostly from the government grant (the average monthly income of livestock owner families was NZ\$ 600-800). Thus, the rules of the shareholding regime were not strictly followed, but rather overlain by the mutual-aid tradition.

The benefit allocation was happily accepted by all participants of the cooperative in 2010 after one year of production and no conflicts arose. The cooperative was planning 
to organise a financial management unit and train a professional accountant from their community for future development.

\subsection{Perceived benefits and difficulties of cooperative activities}

\subsubsection{Benefits}

There were several achievements of the cooperative conveyed by the community informants. They are briefly noted below in order to avoid repetition. For more detail please see the Discussion section below. The four major perceived benefits of the cooperatives are:

1. The condition of the pasture of both villages was perceived to have improved as a result of applying herding rotations. The establishment and development of the joint pasture reduced pasture renting in Hulun gacha that was considered to be leading to severe land degradation;

2. The cooperation and collaborative efforts strengthened the bond among the community members;

3. Surplus labour was released by allocating labour into different units, thus avoiding redundancy; and

4. The traditional cultural activities were promoted significantly. For instance, the Hulun cooperative organised three Nadamu between 2009 and 2010, the most traditional and famous Mongolian cultural gathering.

The partnership between Hulun cooperative and Aolin cooperative was also recognised as being beneficial. The two organisations were introduced to one another in 2009 by Hari association, and have stayed in close contact since then. Three leaders from Aolin cooperative visited Hulun cooperative in early 2010. The leaders from these two cooperatives shared information and experience of organisational management, funding applications, future planning, as well as detailed knowledge of subjects such as sheep breeding. 


\subsubsection{Difficulties}

Overall, there were three main obstacles as summarised by the community informants from each cooperative. In Bayin gacha, the most threatening factor to the cooperative's activities was the disturbance caused by mining activities, including loss of land, pasture degradation and air and water pollution. Furthermore, most community informants were worried about potential government policy influences, particularly economic development oriented policies. Experience taught the community that economic development oriented policies compromise pastoralism for modern economic development.

For Hulun cooperative, the most significant difficulty was the pasture lease issue. Up to 2010 about half of the households in the village owned no livestock due to poverty. The families with no livestock then had to lease out their pasture for income. Most of the pasture tenants were outsiders to the local community, and were not willing to participate in the activities of the cooperative. According to the community members' observations, these tenants had very limited concern about pasture conservation and the condition of the pasture in the long-term, because the term of the lease only lasted from one to five years. As a community interviewee described (2010), "These outsiders are mostly Han people from the neighbouring provinces, and are generally careless about grassland sustainability due to their short term of land use". In order to achieve village-wide collective development, Hulun cooperative aimed to help these families with no livestock to generate income and eliminate land leasing within five years.

The two cooperatives shared a common obstacle - the lack of capable manager/s of their internal finances. As a leader of one cooperative claimed (interview, 2010), "Ideally, some university graduates from the community would be willing to take this job". However he also considered that it was challenging to attract young, educated community members back into the village to carry on pastoralist lives. This is mainly because urban life and office work have become the preference of the younger generations. As a young community interviewee expressed (2010), “... with my newly gained tertiary degree in Business Management, I prefer to find a job in Huhe Haote (the capital city of Inner Mongolia) or even Beijing." 


\section{DISCUSSION AND CONCLUSION}

\subsection{Can the cooperative movement revitalise TEK?}

Two major elements of TEK are being revitalised through the activities of the cooperatives in this case study. First and most significantly is the revitalisation of herding rotations. In Bayin village, a small scale three-season rotation was initiated (covering about 1,000 ha); in Hulun village, a village-wide four-season rotation was initiated (covering about 32,000 ha). This is a critical achievement for the Mongolian TEK revitalisation, especially after 30-40 years of complete abandonment of seasonal rotations. However, compared with the traditional scale of herding rotation, the current scale is still small. Even in the best scenario of Hulun village, in which all families in the village participated, the rotation area is about two to three times smaller than the traditional rotation area according to elder informants (multiple interviews, 2010). Therefore, cross-village cooperation has become a new challenge that confronts cooperative organisations if they want to entirely revitalise traditional herding rotations.

The second most important element of TEK that has been revitalised is the traditional community institutions and community-based resource governance. After the dominance of privatised production practices over the last 30 to 40 years, cooperative practice is rebuilding community cohesion. During the cooperative process of "joiningpasture and working-together" the participating community members resumed their traditional collaborative actions and working relationships. Many community informants claimed that the bond amongst community members has been strengthened since cooperative practices were introduced. The leadership of the traditional community elder has also been revitalised within the framework of the cooperative activities. Elders were traditionally regarded as having noble values, abundant knowledge and authority within the community ( $\mathrm{Da}, 2008)$. The belief in elders' knowledge and judgement, and willingness to accept elders' leadership still commonly exists within the community. In both villages, respected elders were invited when the cooperatives were initiated, and took a leading role in the cooperatives.

Both of the cooperatives operated a consensus decision-making model of communitybased resource governance, which is a process that seeks the agreement or the consent 
of participants, and the resolution to objections (Bressen, 2007; Michael, 1981). This model is similar to the traditional communal decision-making process ( $\mathrm{Da}, 2007$; En, 2005). The board meeting of the cooperative (which can be considered as analogous to the elder group in the traditional system) is the mechanism that makes decisions on a daily basis and determines the need for organising general community meetings. The general meeting (which are the same as the general meeting in the traditional system) is the key mechanism for discussion and decision-making on important issues, such as resource allocation and benefit distribution. For example, in Bayin village, the rules of labour division and benefit allocation were discussed and decided in several general meetings, in which all participants voted. The only difference between the cooperative governance model and the traditional system is the composition of the executive mechanism - usually only elders in the traditional system, but within the cooperative model it is a combination of elders and younger village leaders.

Therefore, the cooperative model integrates the advantages of the traditional mechanisms of resource distribution, job division and benefit allocation, with the contemporary mechanisms such as the voting system. Through this hybrid institution, the elders, with their special influence and skills of problem and conflict solving, play an important role in contributing their knowledge and experience of resource management. For example, elders often act as mediators when a conflict occurs in both the traditional and contemporary institutions. Based on the trust of the elders' knowledge and faith their impartiality, the parties involved in a conflict are usually willing to accept the call for mediation initiated by elders and are willing to listen to elders' opinions. The elders' role in mediating conflicts is difficult to replace with any other person or party. In the meantime, the younger leaders deal with external issues, such as marketing and dealing with other organisations and the government. This hybrid model represents an adaptive development of the community institution - a combination of the traditional and the contemporary institutions. A similar trend has also been observed by other scholars. For example, as Berkes (2008:258) points out, "it is often assumed that indigenous peoples have only two options: to return to an ancient and 'primitive' way of life, or to abandon traditional beliefs and practices and become assimilated into the dominant society. Increasingly, indigenous peoples have been expressing preference for a third option: to retain culturally significant elements of a traditional way of life, combining the old and 
the new in ways that maintain and enhance their identity while allowing their society and economy to evolve".

Based on the local social and ecological conditions of this case study, that have been affected by governmental policies and historical movements (enforced settlement, privatisation, development and conservation policies), a complete revitalisation of traditional practices and institutions is almost impossible. There are also many new challenges that confront the community, such as market forces, an increased demand for cash income, natural climate change, and population growth. Under these circumstances, the most effective response appears to be the revitalisation of certain traditional elements through an adaptive process. To be adaptive and dynamic is a vital attribute of TEK, which has enabled it to survive over thousands of years (Berkes, 2008).

\subsection{Triggers and barriers to the Cooperative}

The cooperative organisations are positioned within a complex system that is influenced by various triggers and barriers. Below, I will summarise the main triggers and barriers to the cooperatives in this case study, and note how these lessons may also apply to other areas of China.

\subsubsection{The triggers}

\section{A. Need and willingness for collective efforts}

As discussed in 3.1.2, the perceived grassland degradation started in the 1980s and has increased since 1999 in the research area. The government diagnosis was that population growth and over-grazing caused the degradation of pasture (e.g. APFB, 2004, 2005; Ministry of Agriculture China, 2010, 2011; National Environmental Protection Bureau of China, 2007). As a result the local government focused on reducing livestock and replacing herders' traditional production with new techniques to deal with the degradation. Differing from the government's theory, herders argued that privatisation and sedentary production are largely responsible for the land degradation. This statement is supported by a number of research findings (e.g. Da, 2007; Da and Zheng, 2010; Jiang et al., 2006; Li et al., 2007; Niamir-Fuller, 2000; Wang, 2005, 2009; Williams, 2002). As Wang (2009) points out, privatisation may increase herders' income in the 
short run, but leaves no space for the sustainability of the grassland. A sedentary lifestyle, on the other hand, has been commonly considered as unsustainable in the region (En, 2005; Da, 2007). Without proper rotation, livestock is only able to feed on a small patch of pasture, and can easily result in over-grazing.

Similar observations are also addressed by scholars elsewhere. Based on a comprehensive review of pastoral herding practices in Africa, Niamir (1998) points out that policies for the settlement of pastoral people and land privatisation are still common in most African countries. Settlement results in managing resources less efficiently due to the loss of traditional knowledge of rangeland management and ecosystems, and the loss of control of traditional pasture use (Farah, 1993; Jacobs, 1980; Niamir, 1998). Meanwhile, the practice of land privatisation has led to accelerated social stratification, expropriation from indigenous peoples' hands, and land degradation across the globe (Lane and Moorehead, 1993; Niamir, 1994).

Land degradation is not the only threat to herders' lives. Frequent natural disasters, especially droughts, have affected the research site significantly. Drought is claimed as the most prominent disaster in the region $(\mathrm{Ba}, 2010)$. Frequent droughts are perceived to have begun in the 1990s and to have increased since 2000. Pasture degradation and reduced hay harvest were direct results of the droughts. Both results made herders' lives extremely harsh and lead to poverty in the community. Traditionally, herders could travel to other locations where were less or not affected by the disaster, and shared the loss with other households who belong to the same Haote. However the settlement policy did not permit this disaster-relief travel, and privatisation destroyed the previous collective relationships among community members. This change reflects that not only the frequency of natural disasters has increased, but also institutional changes instigated by government policies increased herders' vulnerability in the face of the natural disasters. Facing crucial land and resource degradation and social conflicts caused by historical governmental policies, herders looked for solutions in their traditional practices developed from thousand-years-long experience of living on the grassland. The herders themselves initiated the idea of revitalising collective herding rotations and other cooperative activities as they traditionally practiced. 
These perceptions of Mongolian herders are confirmed in the literature. Many studies over the past two decades have pointed to the potential use of the communal management in commons management (e.g. Agrawal and Ostrom, 2001; Berkes et al., 1989; Logan and Moseley, 2002; Niamir, 1998; Ostrom, 1990; Pagdee et al., 2006; Shepherd, 1991). According to Ostrom's (2009: 421) Design Principles for commons resource management, "collective-choice arrangements allow most resource appropriators to participate in the decision-making process". Collective choice agreements assure that the agreed rules will be carried out with reduced costs from sanctions and reduced conflicts (Adhikari, 2005; Adhikari and Lovett, 2006; Agrawal and Ostrom, 2001; Banks and Duggan, 2000). Communal co-ordination is identified as one of the key mechanisms to ensure resilience within traditional pastoral systems facing a wide range of climatic conditions, including episodic devastating droughts (Perrings and Walker, 1995).

\section{B. Law and policy support}

Through this case study, the Cooperative Law and its supporting policies have proven to make a positive contribution to the cooperative development in China. In contrast to previous legislation and policies that acted to reduce community control over resources, the Cooperative Law strengthens and empowers the community as a 'side-effect'57. As argued by many scholars, within the multi-scale social-ecological resource management system, central governmental policy and legislation are powerful mechanisms to change resource management practice and affect lives at a community level (e.g. Ao, 2003, 2005; Ba, 2010; Gao and Song, 2009; Wang, 2009; World Bank, 2006; Young et al., 2006). The central government has the power to either support or undermine participation in decision-making and management actions of institutions at the lower level (Berkes, 2007). This vertical institutional linkage is especially significant in China with its long history of centralised government control (Williams, 2002). The action to support the rural cooperative movement is a step towards community empowerment, even though this might not be the intention or goal of the Cooperative Law. The Cooperative Law grants community level organisations certain power and freedom that has been missing from previous policies (Wang, 2007).

\footnotetext{
57 The original goal of the Law was to bridge the economic and social gap between urban and rural populations in China, but not community empowerment.
} 
This case study presents an example of the positive impact that government policy can have on community development and community empowerment. This kind of success has been claimed in the literature only at a limited number of cases. A review by Folke et al. (2000) notes a few cases in which central government, or similar umbrella institutions, supports local organisation and community-level resource management practices. For example, by recognising and protecting the "tenurial shell", a traditional property rights system in Mexico, the state government supports the traditional belief structure and traditional institutional arrangements of the Huastec people (Alcorn and Toledo, 2000). In Canada, the state government supports the traditional resource management institutions of Cree Amerindians for beaver management in the James Bay area (Berkes, 2000). These examples reflect the critical importance of vertical crossscale institutional linkages for the effective governance of resources and the critical role that central government holds (Young, 2006).

\section{External NGO's assistance}

The importance of external assistance from Hari association has been recognised by the cooperative members in Bayin gacha. The NGO provided information about the Cooperative Law and relevant policies, potential funding sources, and aided the cooperative to connect with other cooperatives and community-focused NGOs in Inner Mongolia. This network has facilitated information sharing and capacity building. As Berkes (2009) and Armitage et al. (2008) define, this kind of network is a typical form of social learning, in which a group of people that have common concerns pursue knowledge through regular or irregular practice based interactions. The social learning process with its focus on learning-by-doing, evaluation, and action modification has been recognised as a key tool for resource management in the face of environmental and socioeconomic uncertainty (Berkes, 2009; Lee, 1993). In this case, the NGO functioned as a bridging organisation between the local government, the community, and other organisations. The bridging role of NGOs is addressed in numerous studies (e.g. Berkes, 2009; Bodin and Crona, 2009; Brown, 2002; Folke et al., 2005; Hahn et al., 2006; Kilduff and Tsai, 2003). As Brown (2001) argues, bridging organisations (usually NGOs) are central to a multilevel management paradigm. They play a critical role in creating and strengthening inter-organisational partnerships that enable cooperative action to deal with problems that cannot be solved by a single agency. Similarly 
emphasised by Folke et al. (2005) is that bridging organisations have the ability to strengthen the capacity for effective governance of multilevel organisations involved in complex social-ecological system management.

In China more broadly, the efforts of NGO networks from inside of China and overseas have enhanced the development of cooperatives, and as a result have boosted community empowerment. More than 1,000 Chinese NGOs have provided services and assistance for rural development (Ao, expert interviewee, 2010). Most of these NGOs are based in cities or towns that have access to government agencies and external organisations, including international agencies and organisations. Between 2008 and 2010, about 20 forums that focused on rural development and cooperative governance were held by NGOs in Inner Mongolia (Ba, interview, 2011). These forums offered a chance for social learning (e.g. exchange information and knowledge among participating organisations), and more importantly to create a network among NGOs and cooperative organisations. The external support from NGOs and other cooperative organisations has the potential to accelerate the development of cooperatives and construction institutions at a community level.

After addressing the triggers of the development of cooperatives in the case study, I have to remind the reader that not all institutional linkages are positive. The government interventions have also had long-term negative impacts on Mongolian TEK. Although the Inner Mongolian communities have a strong will to maintain their traditional institutions, there are various external disturbance and barriers generated at larger and upper scales.

\subsubsection{The barriers}

\section{A. Land tenure}

A common concern in regards to rural development and community empowerment in China is the land tenure issue (Ao, 2003; Cao et al., 2011). Even though the majority of the herders I interviewed were confident about the consistency of the government policy on land tenure, a number of expert interviewees and a few elders expressed their worries about the future of land rights. For instance, when land rights and livestock 
privatisation was enforced in Hulun village in the 1990s, the tenure was granted for a term of 30 years. Currently, there is significant uncertainty regarding the future of tenure following the present contract term. If the herders lose their land tenure, the cooperative would face the loss of their legal standing on the land, and any institutions it has built. Although the terms of land tenure may vary around China, the ultimate control of land ownership is still in the hand of the central government.

\section{B. Governmental intervention}

Apart from land tenure, other potential government influences have concerned the rural population, and conservation and development practitioners in China (Cao et al., 2011). Even though the current government policy is encouraging the development of cooperatives, the policy may change direction if it conflicts with other economic development policies. According to many community informants and expert interviewees, industrial development oriented governmental policies have been dominant since the 1990s. While the grassland conservation policies strictly constrain herders' lives and production (e.g. reducing livestock quota, farm-raising livestock instead of pastoralism, and enforced rest from grazing), the government has granted a large number of mining applications. The total output value of mining activities in 2009 in Xiwu Qi was NZ\$2,200 million, which significantly contributed to local GDP growth (Ba, expert interviewee, 2010). This aligns with the intent of the national Western Development strategy. The community informants were also concerned that the government may intervene in the internal affairs of the cooperative, such as the election process. According to the Industrial and Commercial Corporate Administration Act (1953), since the cooperatives are registered with the Industry and Commerce Bureau, the Bureau has the power to intervene in a cooperative or even close it down.

To date, the central government has taken a dominant role in China (Ao, 2003; Da, 2008). Under such conditions, the extent of control that communities exercise over resource management critically relies on the degree to which government agencies relinquish power to local people. Sharing power has always been a sensitive topic in China, and there is still a long way to go until government authorities and community level institutions have an equal share of power. 


\section{Threshold point of the local TEK system}

Another barrier frequently mentioned by the community informants is the lack of knowledge and experience of cooperation. Most families have lived and worked independently since the privatisation in the 1980s. These families gradually lost traditional working relationships and social institutions. Kinship is the only string that still maintains small groups of herder families bonded together. A family is rarely cooperating with its neighbours who are not blood-related. Therefore, the lack of cohesion among community members is considered to be a major obstacle for largerscale cooperation. Along with economic development, income disparity has appeared in the community. This may block the progress of cooperation as the more wealthy members of the community may not be willing to share their land and resources with the poorer members. The above issues reflect the importance of social capital for successful commons management. As Brondizio et al. (2009: 255) define, social capital is the "value of trust generated by social networks to facilitate individual and group cooperation on shared interests and the organisation of social institutions at different scales". Local networks are often embedded in larger social and ecological systems. Thus, the bonding, bridging and cooperation within and among community groups is essential for achieving cross-scale resource governance at larger scales.

This research finds that, even though knowledge of traditional land use and traditional institutions still exists to a certain extent (mainly within elders), that knowledge has rarely been used over the last five decades. This indicates that the TEK system in the focal community has reached a threshold point, in which permanent loss will be inevitable if no immediate conservation action is undertaken and the elders with key traditional knowledge pass away (Wang, 2007, 2009). Borrowing the concept from ecology, a threshold point, or a critical or tipping point, is the abrupt change of a quality, property or phenomenon in a social-ecological system, or where small changes in the environment produces a large response in the social-ecological system (Beisner et al., 2003; Holling, 1973). Generally, thresholds are the points of social-ecological systems at which abrupt changes occur in the system (Groffman et al., 2006; Huggett, 2005). As many scholars suggest, the occurrence of threshold points signifies the urgent need for immediate conservation actions (e.g. Groffman et al., 2006; Huggett, 2005; Wang, 2009). 


\subsection{The institutional linkages and better governance}

As discussed in Chapter one (section 5.2), resource management is a complex socialecological system, and it needs to be dealt with at multiple scales simultaneously (Berkes, 2010). The institutional linkages, both vertical and horizontal play important roles regarding the success of resource management (Young et al., 2008). In this case study, there is a clear interactive and adaptive process along institutional linkages (see Figure 5.3).

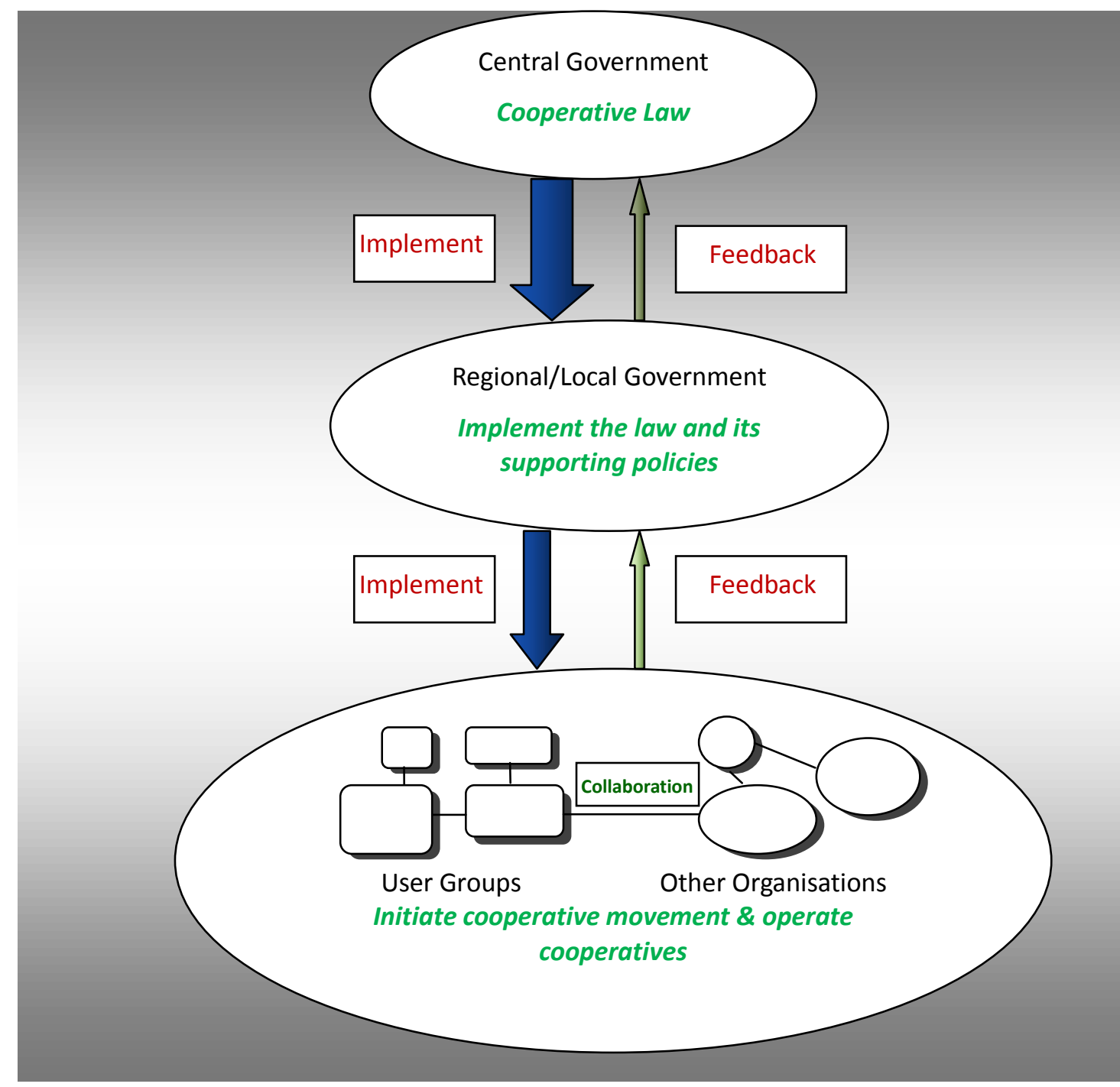

Figure 5.3 The vertical interactions among institutional scales and horizontal collaboration between grassroots level organisations in this case study. Based on Berkes, 2010: 491

As Figure 5.3 shows, the cooperative movement was initiated by community level organisations. The land degradation, poverty and other social conflicts stimulated the 
need for and actions of cooperative activities from the herders. The community participants used their traditional knowledge to revitalise their traditional institutions, including the elder leadership, communal use of resources (pasture, livestock, machinery and infrastructure), working collectively and communal benefit allocation. This cooperative movement occurring at the community level was observed by regional/local governments. Such feedback then was transmitted to central government (see green arrows in Figure 5.3). In this process, central government played its role in receiving feedback from lower levels of institutions, and making decisions accordingly. The emergence and development of cooperative movements urged the establishment of the Cooperative Law and relevant policies. Regional/local governments played their roles in implementing the law and measures, such as providing registration aid to cooperative organisations and other support according to the requirements of the law. In this vertical interplay, every actor has its role. The involvement of all actors and a balanced relationship between them is critically important to any given resource system. The above analysis of vertical institutional interactions is inspired by many studies focusing on institutional arrangements in commons management (e.g. Anderies et al., 2004; Berkes, 2010; Lemos and Agrawal, 2006; Young, 2006). However, to date much of the literature emphasises the decentralisation of resource governance and power devolution, which highlights the one way nature of the actions (see arrows in Figure 5.5 below). The feedback from lower levels to upper levels has not been emphasised in previous research. 

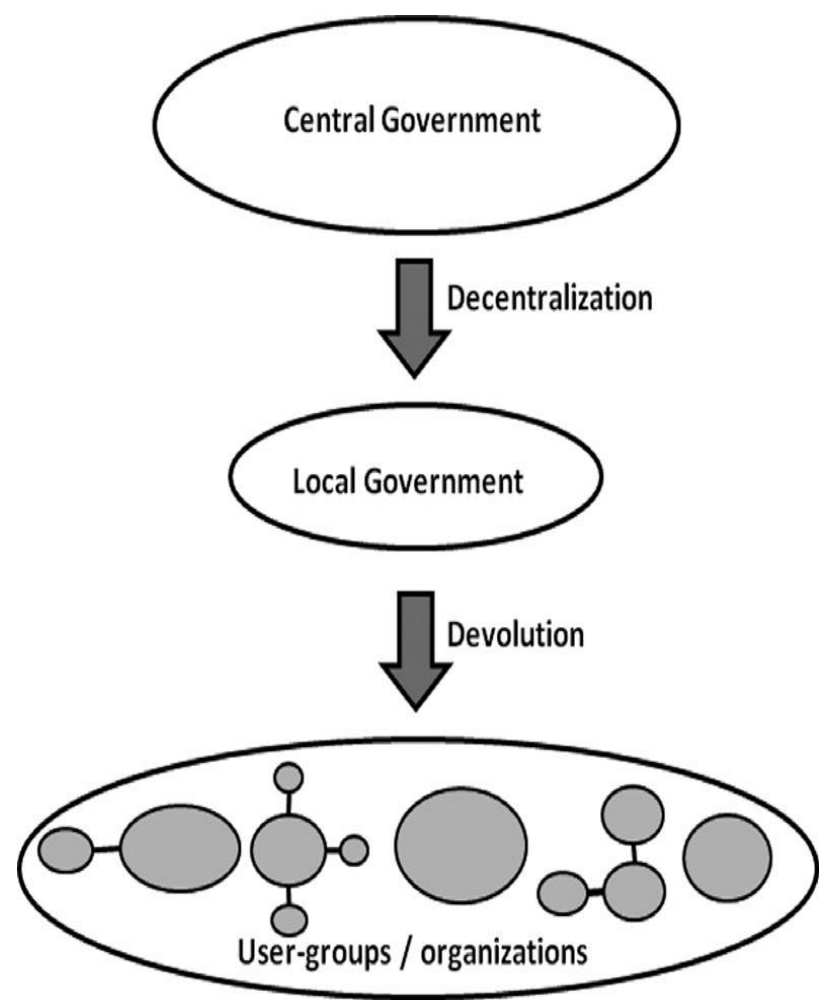

Figure 5.4 Decentralisation and devolution in resource and environmental management. Berkes, 2010: 491

Alongside the vertical institutional linkages, horizontal linkages built a network of collaboration at the grassroots level (see the bottom circle of Figure 5.4).

Through this network, multiple cooperative organisations and NGOs from different regions were connected. For example, the local NGO (Hari association) connected Aolin and Hulun cooperatives, and they stayed in close contact. Both cooperatives also attended two regional cooperative conferences, in which five NGOs and 12 cooperatives participated. Through such practices, a network has been built up of horizontal, crossorganisational collaboration.

However, horizontal collaboration at a wider level, specifically across-village herding collaboration, has not yet been achieved. As discussed in section 4.1, several key informants (elder interviews and three cooperative leaders) emphasised that multivillage cooperation is vital for revitalising the traditional large scale herding rotation. This is challenging to establish because the villages have been separated for several decades and it is not easy to revitalise the traditional communal use of the pastures at this scale. Based on the current practice of the cooperatives, they expressed confidence 
in the possibility of multi-village cooperation in the near future. To date, no horizontal collaboration at other levels of organisation, such as between local governments within the region or country was perceived. There is a great potential to build such collaboration that enables government agencies across regions to share information and potentially provide a foundation for cross-regional cooperative practice at the community level. As many scholars address, horizontal institutional linkages are important because they enable cooperation across space, facilitate social learning and assist risk management (Berkes, 2009; Brondizio et al., 2009; Ostrom, 1990, 2009). Both vertical and horizontal linkages are equally vital to achieving robust resource management within the framework of social-ecological systems.

This case study illustrates that Bayin community possesses traditional knowledge and institutions for sustainably managing grassland, and is willing to take responsibility for resource management. As many community members stated, as long as the government does not intervene, they have confidence in their "traditional wise use" of the grassland.

\subsection{The limitation of this research \& possibilities for future research}

The findings of this case study has potential applicability in other areas of China, however the application of the findings more broadly has to be considered carefully. As such, there are several main limitations of this research. First, the cooperatives in the case study and in wider China have only been active for a decade. The impact on traditional institutions and the wider aspects of TEK and community empowerment require on-going monitoring and further research. Second, the implementation of the Cooperative Law and assisting policies may vary in different locations. Local governments will play a critical role in determining the local policies. In turn, it could lead to different cooperative development at the local level, and ultimately have different effects on TEK and its revitalisation. Third, TEK is not a homogenous system of thought; TEK may vary significantly among different cultural groups and ecosystems. Thus, the conclusion from this research in Inner Mongolia is not necessarily applicable to other locations and other TEK systems even within China. Another concern is that, as mentioned in 3.1, when the community informants (especially the elders) may have romanticised the past. This trend has been observed and discussed by multiple scholars 
(e.g. Bernard, 2010; Cruickshank, 2002). These romantic opinions may generate nostalgic bias to the research results.

So far, this case study has been the first research that focuses on the effects of government policy and legislation on TEK and TEK conservation in Inner Mongolia and China. It is hoped that it would inspire further research in this field and in other areas of the world.

\subsection{Conclusion}

The traditional institutions and practices of herders in Inner Mongolia were adversely affected by government policies between the 1960s and the early 2000s. Even though the local knowledge of traditional resource use and institutions still exists, the practice of the knowledge has stopped for decades. The local TEK system has then reached a threshold point, in which permanent loss of the knowledge will be inevitable if no immediate conservation action is undertaken.

The emergence of cooperatives has been a grassroots level movement. Three or fourseason rotation practices have been revitalised within the cooperatives. This is a critical achievement of Mongolian TEK revitalisation, especially after the abandonment of three and four-season rotations for 30-40 years. Certain elements of traditional community institutions and community resource governance have also been revitalised. During the process of "joining-pasture and working-together", the participating community members resumed traditional collaborative relationships, strengthening the bond amongst community members. The cooperative model combines the advantages of both traditional (e.g. elder's leadership; communal benefits sharing mechanism) and contemporary institutions (e.g. young learship) and is an adaptive development of the community institutions.

In this case study, both vertical and horizontal institutional linkages applied. In the vertical interplay, the community initiated the cooperative movement and the regional/local government observed and transmitted feedback to the central government. In turn the central government responded to the community level 
movement. The establishment and implementation of the Cooperative Law and its supporting policies provided positive support for the development of cooperatives. The horizontal linkages involved building a network of cooperatives and NGOs at the grassroots level. Participating organisations shared information of relevant government policies, experience of managing organisations, capacity building, and future plans through this horizontal network.

This research illustrates that there is great potential for the revitalisation of Mongolian TEK under the current cooperative movement. The two communities have shown that the development of cooperatives creates opportunities for community autonomy over land use and resource management. This empowers the community in its role in multiscale resource governance. However, the cooperative movement is still in an early stage in China, and its long-term impact on revitalising TEK and empowering the community in particular requires continuous monitoring and further research. 


\section{CHAPTER SIX}

\section{TYPOLOGY APPLICATION}

\section{INTRODUCTION}

In Chapter Three and Four, I presented the typologies of TEK threats and TEK conservation actions, and explained the typologies with examples from the survey and the literature. In this Chapter, I will use the case study from Chapter Five to demonstrate how the typologies can be applied to situation analysis and decision-making in realworld cases.

\subsection{Why study the application of the typologies?}

The importance of having a standard classification system in the TEK field has been discussed in previous chapters (see Chapter 1, section 4.5; Chapter 3, section 1.3; Chapter 4, section 1.2). In order to avoid repetition, I will only highlight two of the most important properties of the TEK typologies here: 1) the typologies provide a useful means for TEK conservation actors to identify the threatening factors to TEK, and a toolbox of possible conservation actions; and 2) they provide a common language for TEK conservation actors to communicate and share information and experience. By applying the standard classification system, conservation actors may also be capable to assess and compare conservation outcomes across regions and projects.

As Salafsky et al. (2008:12) address, "the true test of any classification scheme is whether it can be meaningfully applied to real-world situations". In Chapters Three and Four, I provided numerous examples from the survey and literature to demonstrate the categories and sub-categories of the typologies. However, one of the limitations of using these examples is that they often lack certain details to construct a comprehensive, indepth analysis. To apply the typologies to first-hand research and in-depth analysis for the testing purpose then is crucial. By applying the typologies to the Inner Mongolia case study, I will present a model of TEK threat analysis and conservation action design. This model aims to assist researchers and conservation actors to apply the typologies to their cases, and improve the TEK conservation outcomes. This exercise is also critical for 
the issues with TEK in Inner Mongolia. In applying the typologies I hope to gain a better understanding of the threats that TEK faces in Inner Mongolia, and a more systematic view of possible conservation options.

\subsection{Why choose this case study?}

There are three main reasons for choosing this case study for advanced analysis in this chapter. First, as I discussed above, it is first-hand research, in which I was able to collect data myself, and to develop understanding of the local context through multiple research methods. Comparing the data gathered via questionnaires and interviews, I found the data generated from first-hand research is more comprehensive. For example, through participation in and direct observation of informants' daily lives, I had a chance to gather the information that filled the gap between "stated behaviours" in questionnaires and interviews and "actual behaviours" in real life. In this way I was able to ensure a more comprehensive understanding of the specifics of TEK threats and current TEK conservation actions in the case study locations and map these onto the typologies.

Second, Inner Mongolia is a significant location in terms of indigenous culture and traditional knowledge in China and in the world. The dramatic loss of Mongolian TEK has been reported by many scholars (e.g. Eer, 2007; Fernandez-Gimenez, 2000, 2002; Hasi et al., 2011; Song, 2010; Williams, 2002; Wu, 2005; Xue, 2009; Ye, 2009). However, under the socio-political context in China, the recognition and conservation actions of indigenous rights and TEK have been undertaken to only a very limited level $\mathrm{Xu}$ and Melick, 2007; Ye, 2007). Due to the combination of rapid loss and limited conservation efforts, Mongolian TEK has been in a critically endangered position, which requires wider attention and immediate actions to conserve. To date, the TEK research in Inner Mongolia has largely focused on ethnobotanical knowledge (e.g. grass varieties, grass composition and arid tree ecology) (Eer, 2007; Hasi et al., 2011; Song, 2010; Wu, 2005; Xue, 2009; Ye, 2009), with a very limited number of studies of traditional herding practices and resource management (Ao, 2009; Tang and Gavin, 2010). Studies of wider aspects of TEK, such as traditional institutions and traditional beliefs are missing from the current literature. In addition, the conservation of TEK in Inner Mongolia has 
received no attention in the literature. The discussion of TEK and recognition of the importance of TEK in Inner Mongolia and China more broadly has only recently been gaining academic attention; and TEK conservation has not been promoted, especially given the social-political pressure for modern development in China currently (Ao, 2009; Da, 2007; Da and Zheng, 2010). Under these circumstances, the TEK typologies may provide a comprehensive approach for understanding TEK threats and possible TEK conservation actions in Inner Mongolia.

Finally, the precarious status of TEK in Inner Mongolia is similar to in many other locations around the globe, especially in Asia, Africa and South America. Therefore, the Inner Mongolia case study may provide inspiration for TEK conservation projects in other regions.

\subsection{Aim \& Objectives}

This chapter aims to demonstrate how to apply the TEK typologies into real-world situation analysis and decision-making. The chapter will provide a model of TEK threat analysis and TEK conservation action design, as well as assessment of TEK conservation actions.

The research objectives of this chapter include three themes:

1. Using the threat typology to identify and analyse TEK threats in the research region, and compare it with current published knowledge of threats.

2. Using the action typology to identify and analyse current TEK conservation actions.

3. Using the action typology to make recommendations for future conservation actions.

\section{TEK THREAT IDENTIFICATION \& ANALYSIS}

This analysis is based on the results from multiple research methods, including semistructured interviews with multiple groups (i.e. Mongolian herders, experts on 
grassland ecology and Mongolian traditional culture, local NGO workers and governmental officials), focus group discussions with Mongolian herders, and participant observation. By synthesising the data provided by different informants and from different research methods, five major threats to traditional knowledge and practices have been identified. I will discuss each of these threats in detail, then conceptualise them into the threat typology's categories.

\subsection{The change of traditional communal nomadic pastoralism}

\subsubsection{A brief review of the issue}

Prior to the 1960s, herders in the research site retained their traditional nomadic life over a thousand of years (Ao, 2003, 2005; Da and Si, 2006). The grand commune ${ }^{58}$ was introduced into the research region in the early 1960s (Da and Zheng, 2010). All previously collectively owned land and other resources by Mongolian herders were claimed as state asset, and managed by the commune. All herders were assigned jobs by the commune leaders, who were appointed by the local government (multiple interviews, 2010). As many informants $(n=35)$ recalled, the government-appointed leadership replaced the traditional elder's leadership in the 1960s (see more details in Chapter 5, section 3.1.1).

Based on the government's perception that nomadic lifestyle is backward and undeveloped, the settlement policy was implemented in the late 1970s by the government authority (Wang, 2009; Williams, 2002). The local government established a medical centre, veterinarian service and a primary school in the nearby sumu (equivalent to township) to encouraged herders giving up traditional nomadic lifestyle and stay around the sumu all year long. The full settlement of the community was achieved under the aid of privatisation policy later. In 1983, the previously communally owned livestock was redistributed to individual households based on the size of household (roughly about 50 livestock per person) (Li, interview, 2010). In 1984, the user right of pastures was distributed to household level according to their livestock shares (Li, interview, 2010). Since then, the state government recognised herders' ownership of livestock, and their exclusive user right of the land and pasture they lived

58 A part of the property nationalisation process in the 1960 s in China. 
upon. The privatisation process was finalised in the 1990s when the majority of the households fenced off their lands ${ }^{59}$ (Aote, interview, 2010). The completion of the privatisation process indicates the end of Mongolian traditional nomadism. Since then, all families in Bayin gacha have settled down and lived in their fixed locations only. The traditional four-season rotation became impossible, and the knowledge and skills associated with four-season rotation, such as determining rotation time and choosing rotation routes, has been degraded, especially with younger herders (Te, interview, 2010; participant observation, 2010). For example, $63 \%$ of the community informants (including interviewees and participants of focus group discussions) in the village are under 40 years old, and have never experienced four-season nomadic pastoralism. Their knowledge of the traditional nomadism limited to elders' descriptions of the past practices.

\subsubsection{TEK threat analysis}

Applying the above issue to the threat typology, two matching categories of direct threats are identified - change of traditional livelihood practices and loss of traditional rights.

\section{1) Change of traditional livelihood practices}

In this case, the sedentarisation and privatisation of land and livestock led to the abandoning of Mongolian traditional four-season herding rotations and the communal production system. In this transformation process, sedentarism replaced the nomadic lifestyle, and privatised production replaced the communal production system. The Mongolian traditional livelihood practices have changed significantly. The sedentarisation and privatisation are two basic attributes of the modern, or in another word, the Westernised production system (Steinfeld et al., 2006). The issue therefore may be further categorised as use of Westernised primary production systems ${ }^{60}$ in the typology.

\footnotetext{
59 The fencing process was mainly enforced by government mandate.

${ }^{60}$ Text in bold indicates it is a matching category from the typology; text in bold and italics indicates it is a matching sub-category from the typology.
} 


\section{2) Loss of traditional rights}

When the state claimed its ownership to all land and resources in the 1960s, it deprived Mongolian herders' traditional rights of their land and resources (Ao, 2009). Even though herders remained to live on their traditional land, the loss of ownership undermined their rights and possibilities of maintaining their traditional lifestyle and practices (Chen, 2009; Da and Zheng, 2010). Furthermore, under the enforced settlement, Mongolian herders lost their traditional rights of herding rotations and traditional access to wider pastures (Aote, interview, 2010). This can be further categorised as loss of traditional land use and loss of access to traditional resources according to the TEK threat typology.

The main underlying cause of TEK change in this case appears to be government policy, which was identified as a cause of TEK change by all community and experts informants. The policies of settlement and privatisation are the fundamental driving forces of Mongolian herders' sedentary lifestyle and privatised production (Ao, 2003, 2005; Da, 2007). Within this transformation process between the 1960s and 2010, the Mongolian herders did not have a chance to decide whether or not they can keep their traditions and lifestyle (perceived by multiple interviewees and group discussion participants, 2010). The top-down resource governance provided a distinct barrier to community involvement in decision-making (Wang, interview, 2010). On the contrary, all the official interviewees $(n=9)$ felt these changes had improved herders' life. This attitude reflects the mainstream trend of governmental policies in China (Wang, 2009).

\subsection{The loss of land or land use caused by mining activities}

\subsubsection{A brief review of the issue}

As the response to a predominant national policy - the Great Western Development Strategy ${ }^{61}$, the local government has emphasised on increasing the contribution to GDP growth and industrialisation in the region since 2000 (Da and Zheng, 2010). The importance of pastoral production has been undermined by the economic development pressure in the research region (Wu, interview, 2010). Industrial development,

61 The policy was implemented in 2000 with the aim to fasten the economic development in western regions of China, which are mainly inhabited by indigenous minorities. The main targets include the development of infrastructure, promotion of education and enticement of foreign investment. 
especially mining activities have been favoured and supported by local authorities. According to the Xiwu Qi Annual Statistic Report of Economic and Social Development 2010 (Xiwu Qi Statistics Bureau, 2011), the total GDP of Xiwu Qi in 2010 was NZ\$1.72 billion, in which the industrial component contributed NZ\$1.392 billion (84\% of the total GDP), while the pastoral factor only counted for NZ\$ 0.164 billion (9.7\% of the total GDP). The report also emphasised the "great achievements" of increasing production of coal (38.68 million tons, annual increase rate was 51.6\%), electricity (694.79 million KW, annual increase rate was $277.2 \%$ ) and cement ( 0.477 million tons, annual increase rate was $32.4 \%)$. Other mining products mentioned in the report are lead (15,150 tons), zinc (36,880 tons), copper (10,800 tons) and zinc ingots (105,375 tons). Based on one assessment (Ren, interview, unpublished data $\left.{ }^{62}, 2010\right)$, there are more than a thousand mines in Xiwu Qi, including coal, iron, zinc and other metal mines. The developers of these mines are mostly outsiders. All informants, apart from local official interviewees, agreed that mining has been the most destructive factor to pastoral practices and local lives in the research area.

The land occupation for mine development has happened at a significant pace. In Xiwu Qi, many herders were forcibly removed from mining sites, and relocated into town under local government's arrangements (Ren, interview, 2010; Da, interview, 2010). In Bayin gacha, 16 households have been removed by mining activities since 2000 (Aote, interview, 2010). The local government paid NZ\$210 per hectare as compensation to these mining migrants, but there was not any supporting plan for their future living (Ao, interview, 2010). Herder families who have not been relocated have to face a number of serious challenges caused by mining, such as dust pollution, groundwater usage and discharge pollution. These issues have caused ground vegetation degradation and livestock death. Most of the adjacent areas to mines became un-useable or un-accessible for herding purpose (Ao, interview, 2010; participant observation, 2010).

\subsubsection{TEK threat analysis}

The above issues can be categorised as loss of traditional rights using the typology. For the herders who were removed from their pasture, they lost the land tenure and their

\footnotetext{
62 Due to the political sensitivity of mining issue in Inner Mongolia, research on such topic has been eliminated from publication and media release under governmental control.
} 
traditional land use. For the herders who are facing mining stress on their pasture, they lost their traditional land use and /or the access to traditional resources.

There are two layers of underlying threats under this issue. The first layer, which is the most immediate cause of the mining development, is government policy that aims to increase economic growth through industrialisation. The policy recognises the priority of industrial development, and provides support to mining activities (Xiwu Qi government, 2006). For example, according to the Xiwu Qi Regional Economic Development Plan (Xiwu Qi government, 2010), in accordance with the national Grand Western Development Strategy, the main target of the local development between 2006 and 2010 was to develop Xiwu Qi as a supplying base of coal, electricity and non-ferrous metals by maximum utilising of natural resources in the region. By implementing these policies, it directly undermines herder's rights and well-being.

The second layer of the underlying threat, which is the cause of development-oriented governmental policy, is the national economic development pressure. The economic growth has been one of the most important driving forces of Chinese government policies nationwide since the 1980s along with the process of the Chinese economic reform (Wang, 2009). The reform has also led to the establishment and implementation of a series of different legislative initiatives, such as $<$ The State-owned Industrial Enterprise Law $>,<$ Chinese and Foreign Cooperative Venture Law $>$, $<$ Foreign Investment Law $>$ and <Compulsory Education Law> (Jin, 2002). Since the late 1970s, the Chinese economy has steadily grown (Jin, 2002). A goal of maintaining a steady annual GDP growth of $8 \%$ has been targeted by the central government since the 1990s (Zhang, 2005). However, numerous environmental and social issues have arisen along with the significant economic growth (Yang, 2005). In turn, the Chinese government has been criticised as compromising environment and social equity for economic growth (Da and Zheng, 2010). 


\subsection{The weak social cohesion when dealing with stress}

\subsubsection{A brief review of the issue}

Prior to the 1960s, the traditional institutions, which were based on kinship and elders' leadership, functioned over a thousand years (Enkhee, 2009). However, the grand commune movement in the 1960s-1970s ended this traditional system (Ao, 2003). Instead, the commune executed the state ownership over the land and resources, and all herders were assigned jobs by the commune leaders. The commune leaders were appointed by the local government, and the leaders were often Han Chinese and from outside of the community. The grand commune era constructed the model of "modern leadership", which was characterised by young, educated people chosen for positions of power by the government, as opposed to the traditional elders' leadership (multiple interviews, 2010). This model of youthful leadership has been carried out until the present time (participant observation, 2010).

As a result from the collapse of the traditional communal production system, the community cohesion which was co-developed with the communal production system has been diluted (Enkhee, 2009). For example, in the past, the equal and dual-beneficial relationships among herders allowed them to collectively cope with natural disasters (Ao, 2005; Enkhee, 2009). Free rotations between Haotes to mitigate the stress of natural disasters were common, and herders shared the risk and cost of natural disasters (see more details in Chapter 5, section 3.1.2). However, since the privatisation, herders are stuck to their contracted lands and work on individual family units. The traditional cooperative disaster mitigation mechanisms (i.e. herding rotation and dual aid among Haote members and between Haotes) have been eliminated. This is because travelling to or through other family's pastures for rotations has become impossible. As a result, individual herder families have become more and more vulnerable to extreme weather conditions.

Apart from the natural stress, the community has been constantly challenged by external political and social stress. For instance, when a mining company accesses one family's land, the family has to face the stress alone without much community support. The official village committee, as the grassroots institution of the governmental 
authority, will not stand up against mining activities which is favoured by the local government (multiple interviews, 2010).

\subsubsection{TEK threat analysis}

Applying the above issue to the threat typology, they can be categorised as loss of traditional institutions. The commune movement completely changed the traditional resource governance. It replaced the kinship-based collective resource ownership with state ownership; it replaced traditional elders' leadership and conflict resolution mechanisms with the appointed community committee and its authority.

Once again, the underlying cause of this threat is government policy. As I discussed above, the grand commune movement was enforced by the state policy and left herders no choice to maintain their traditional institutions. To achieve the state control of all land and natural resources was the target of Chinese governmental policies between the 1950s and the 1960s (Da and Zheng, 2010).

\subsection{The grassland degradation and increased cost of production}

\subsubsection{A brief review of the issue}

The grassland degradation has been perceived and reported since the 1980s (e.g. Ao, 2005; Da, 2003a, 2004; Williams, 2002; Yi et al., 2009). All the community ( $n=132)$ and expert $(n=11)$ informants of the Bayin case study strongly linked the grassland degradation with sedentary lifestyle. As many community informants emphasised that, without proper rotation, the pasture has been over-used and had no chance to recover from grazing. In order to cope with the grassland degradation and frequent droughts, herders started to preserve hay for winter use since the 1990s. Every household had to fence off a grazing-free zone as a hay reserve. However, in most the cases, herders had to buy extra fodder from the nearby market. The hay harvest and storage, and the purchase of extra fodder hugely increased the cost of herding production (multiple interviews, 2010; participant observation, 2010) (see more details in Chapter 5, section 3.1.2). 


\subsubsection{TEK threat analysis}

Applying the above issue to the typology, it can be categorised as change of environment and natural resources. In this case, the resource (pasture) degradation was a major problem that challenged Mongolian traditional practices. The pasture degradation forced the herders to reduce the number of livestock, especially horses ${ }^{63}$, and start to carry out non-traditional livelihood practices, such as hay reserve and purchase of fodders.

The underlying cause of this TEK threat is again inappropriate government policy. As many informants pointed out, which is supported by a number of scientific studies (e.g. Ao, 2005, 2009; Da, 2003b, 2004; Da and Zheng, 2010), sedentary lifestyle pushed by central and regional government policies is largely responsible for grassland degradation. For instance, Ao (2009) notes that Mongolian traditional nomadism is a result of knowing and adapting their livelihood activities and social practices in accordance with the attributes of grassland ecosystems in the region. The forced settlement policy of the government has had profound negative impacts on Mongolian herders' economic independence and traditional cultural identity. The cultural and ecological rationality of nomadism has been largely ignored by the settlement policy.

\subsection{The absence of younger generations from the community caused by the formal education}

\subsubsection{A brief review of the issue}

The free, compulsory primary education system has been implemented in the region since the 1970s (Li, interview, 2010). All children between seven and fifteen years old have to enrol in schools (participant observation, 2010). Before 2002, while the middle school aged students had to study in town; there was a primary school in the nearby sumu (township). Most primary school children were able to travel 45-80 minutes on horse-back to attend the school daily or weekly (Wu, interview, 2010). However, with the aim of promoting urbanisation in the region, the local government applied a policy

\footnotetext{
${ }^{63}$ Horse is a cultural symbol of Mongolian populations. However, it requires much more fresh grass to feed compared with other livestock, such as sheep, goat and cattle. It has become too expensive to maintain a big herd of horses for most the families in the community.
} 
called eliminating township and combining towns as an important component of urbanisation processes in 2002 (Xiwu Qi Government, 2006). By implementing this policy, more than half of the townships in the Xiwu Qi region were eliminated. All social services, including primary schools, at the township level were removed. Since then, all school aged students have to go to Xiwu city for education. While the school students stay in Xiwu, at least one adult from their families has to stay in the city to look after them (participant observation, 2010). After middle school years, most students would continue their high school education in Xiwu (multiple interviews, 2010). And some of them are able to take tertiary education in bigger cities. For those who take higher education in Xiwu or other cities, most of them prefer to find a job in town or cities, instead of having a herder's life (participant observation, 2010). As a new graduate from a university stated, "My major in university was English literature. There is not much I can do in our community. I'm looking for an English teacher position in Xiwu, or in Huhe Haote (the capital city of Inner Mongolia)".

\subsubsection{TEK threat analysis}

Within the threat typology, the issue posed by the formal education system can be categorised as loss of pathways of TEK transmission. In this case, the younger generations stay in town for education. It usually takes them more than nine years away from their families and communities. These young students only spend summer and winter holidays at home (about two months a year) (Wen, interview, 2010). This absence of young generations from their community cut off their possibility of learning traditional knowledge and practices from their families and other members of the community. It also is common that these young students complain about being bored at home, and miss the town life (participant observation, 2010). This is more likely to be caused by dominant society's cultural influence that generated from the contact with other cultural groups, such as Han. It also significantly reduced their interests in the herding life and their incentive to learn TEK.

The underlying causes of this threat appear to be the implementation of governmental policy (compulsory education policy) and contact with other cultural groups. The compulsory education policy enforces school aged young Mongolians to spend more 
than nine years in town. During this period of time, they have more access to a modern lifestyle and modern knowledge systems than their traditional knowledge system and culture. Meanwhile, by living in town, they have been influenced by mixed cultures from other cultural groups. All these influences often drive young Mongolians' worldview and life preference away from their traditional ones.

\subsection{The TEK threats causal web \& Discussion}

Based on the TEK threat analysis above, I developed a diagram that illustrates the system of direct threats and underlying causes in this case study below. It provides a visual aid for the reader to understand the causal web of TEK threats. 


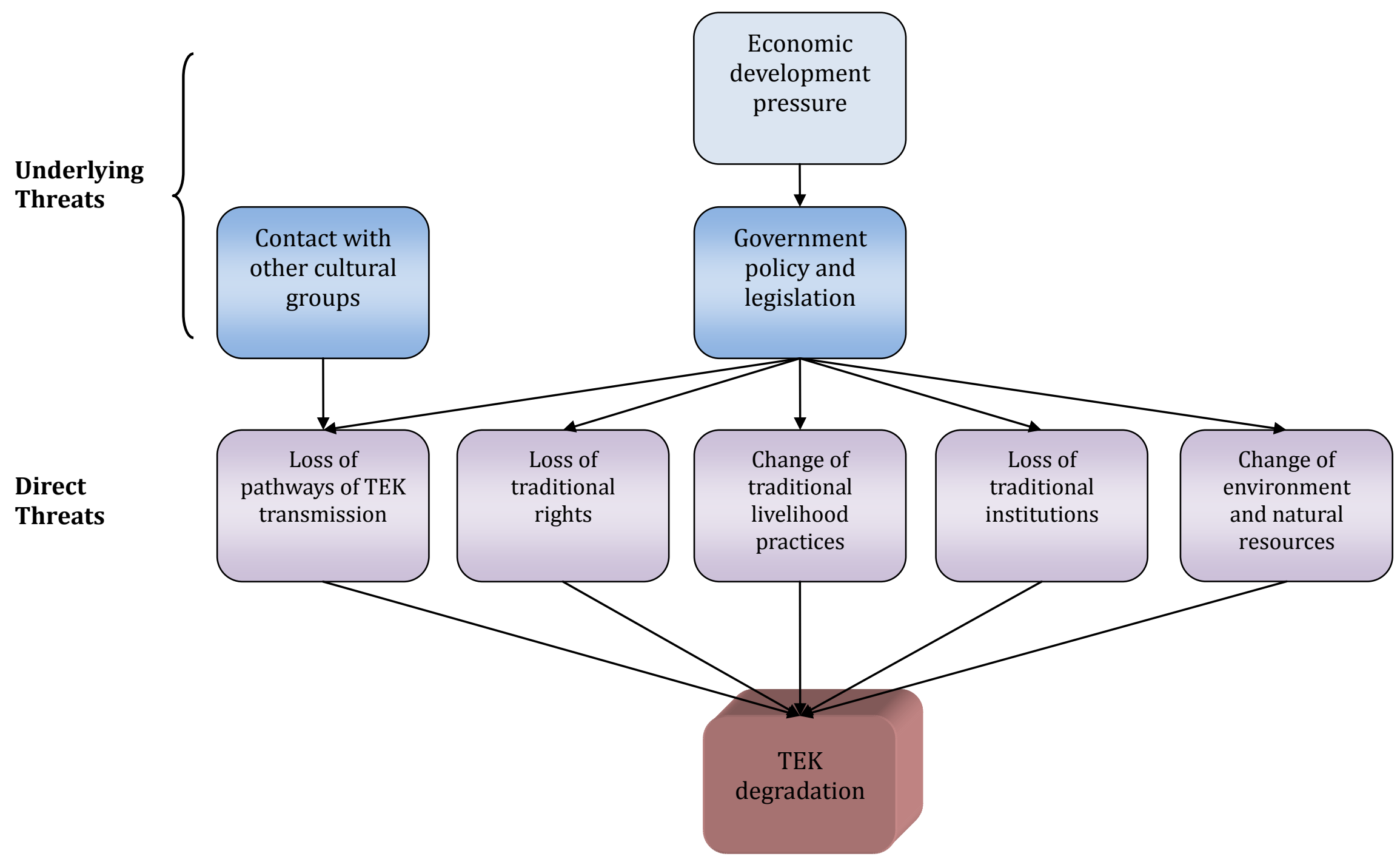

Figure 6.1 The causal web of TEK threats in Bayin case study 
From the above figure, we can see there are three layers of the causes of TEK degradation in the research site. At the first level, five direct threats (i.e. loss of pathways of TEK transmission, loss of traditional rights, change of traditional livelihood practices, loss of traditional institutions and change of environment and natural resources) took place at the local level and led to TEK degradation in the community directly. At the second level, two underlying threats, including contact with other cultural groups and government policy and legislation took place at both the regional and national levels and led to TEK degradation through causing direct threats. At the third level, national economic development pressure influenced government policy and legislation at both regional and national levels.

This analysis illustrates the nature of the TEK threats web as a multi-level system, where multi-scale interactions exist and multiple institutions are involved. The vertical institutional linkages are shown clearly in Figure 6.1, from the direct threats that occurred at a local level, involving community institutions, to the underlying threats that occurred at regional and national level, in which regional and national authorities and other organisations are involved. In this case study, TEK degradation at the community levels is a result of top-down resource governance. As many scholars point out (e.g. Agrawal, 2003; Berkes, 2010; Brondizio et al, 2009; Cash et al., 2006; Ostrom, 2009), resource management requires institutional arrangements that facilitate the cooperation, mediation, translation, and negotiation of information and knowledge within and across levels (Brondizio et al., 2009). The threat analysis of this case, however, demonstrates the failure of such cross-level cooperation. The top-down decision-making system leaves no room for institutions from lower levels, especially from the community level to participate. On the contrary, government policy and legislation have significantly affected the practices at the community level, and are largely responsible for the loss of TEK.

Comparing the above results with previous studies in the region helps in evaluating the feasibility and usefulness of the typology. Only two previous published studies have focused on TEK in the research region64 (Hasi et al., 2011; Hu, 2009). The main target of these two studies was collecting and recording ethnobotanical knowledge. The research

64 The Xilin'guole Meng of Inner Mongolia, where Xiwu Qi belongs to. 
recorded local botanical taxonomy, and the variation of TEK among different informant's groups. There are several threats to Mongolian TEK mentioned by the researchers in general, namely government policy influence, immigration and other social change, and land and resource degradation. However, due to the ethnobotanical focus of these studies, the details of TEK threats have not been discussed. Even though both studies noted the loss of TEK and suggested TEK conservation as a priority, they did not provide further discussion on the topic. In wider Inner Mongolia, TEK research has been carried out since the 1990s. Similarly to the research in Xilin'guole Meng, the majority of these TEK studies focus on ethnobotanical values of Mongolian TEK and traditional grassland management techniques (e.g. Bao et al., 2009; Erde, 2007; Liu et al., 2009; Jimu et al., 2009; Song, 2010; Mu, 2007; Wu, 2005; Wuren et al., 2009). Other aspects of TEK, such as traditional institutions and belief system, have not been targeted. The causes of TEK degradation have been identified in a general manner without much detailed analysis and discussion. A systematic study of TEK threats in Inner Mongolia is missing from the current literature. The Bayin case study and above analysis provide the first systematic approach for analysing TEK threats in the research region. By utilising the TEK threat typology, this approach ensures a more thorough understanding of all possible threats and underlying factors of TEK degradation in Inner Mongolia.

\section{THE CURRENT TEK CONSERVATION \& ASSESSMENT}

\subsection{The current actions}

In 2009, encouraged by the implementation of the China Farmers' Professional Cooperative Organisation Law (Cooperative Law) ${ }^{65}$, a community-based cooperative organisation was established, in which six families participated (Aote, interview, 2010). The primary focus of the cooperative was to form a collective working group and revitalise herding rotations. These participant households tore down the fences of their properties and joined their pastures and herds. The joint pasture of six households counted for about 1,333 ha, in which a small-scale herding rotation occurred (multiple focus group discussion participants, 2010; participant observation, 2010).

\footnotetext{
65 The Law was enacted in October 2007. It recognises the legal title of farmers and herders' cooperative organisations, and provides supporting policies to encourage cooperative activities.
} 
The cooperative members also divided up jobs based on experience. The most experienced herder and his family were selected to be the chief shepherd. With the assistance of another two families, the chief shepherd family decided where to graze, when to rotate and where to rotate to. Another three families mainly carried out jobs like hay harvest, livestock nursery, and collecting firewood and cow dropping for heating purpose. In autumn, the hay harvest season, most of the cooperative members worked together in the hay reserve and left one or two person/s to graze the herd. Likewise, in spring, most of the members were engaged with lamb and calf nursery (multiple focus group discussion participants, 2010).

According to the community informants who participated in the cooperative $(n=40)$, the most significant achievement of the cooperatives is that it provided a chance to revive herding rotations. The joint pasture was used by all of the six participating families; a three-season rotation has been achieved. However, for revitalising the traditional fourseason and large scale rotations, a minimum of 3,333 ha of pasture is required (more than the total area of ten households at least) (Aote, interview, 2010). According to the community elders, ideally, it will require the whole village, even multiple villages to participate in the cooperative.

Many community informants emphasised the importance of taking collaborative actions to reduce the cost and risk of individual production, and to achieve a better market position through the cooperative practices. For instance, six participating families already shared machinery and labour during the hay harvest season in 2009 (Aote, interview, 2010). A family voluntarily provided a haymaker for the cooperative use without charge. During the development of the cooperative, the traditional community cohesion has been strengthened, and traditional institutions (i.e. elder's leadership, collective working relationships and decision making processes) have had a chance to be partly revitalised (see more details in Chapter 5, section 3.3.1 \& 4.1).

There are two external factors that have facilitated the development of the cooperative: 1) the implementation of the Cooperative Law and its supporting policies; and 2) the assistance from a local NGO - Hari Gentai Green Animal By-products Development 
Association (Hari association) ${ }^{66}$.

The Cooperative Law recognises the legal corporation titles of cooperatives and provides policy assistance to cooperatives' development, such as registration aid and tax reduction or exemption (Guo and Song, 2009). Although the fundamental goal of the law is to enhance rural economic capacity, which is in accordance with the overall economic development policy in China (Young et al., 2006), the law may create space for rural capacity building and community empowerment. For example, within a cooperative organisation, participating households have been able to carry out some traditional herding practices (e.g. small-scale rotation), and partially revitalise the traditional institutions behind the practices (e.g. collective working relationships and elder's leadership).

The NGO provided useful information of the supporting measures of the Cooperative Law, potential funding sources and potential network to the cooperative. For example, the NGO introduced the cooperative with another cooperative in Inner Mongolia and facilitated their experience exchange. The two cooperative organisations, which were connected through the NGO's aid, shared information and experience of cooperative operating, administrative issues and funding applications and management. The NGO also aided the cooperative to connect with potential sheep breeders from other regions in Inner Mongolia (Ba, interview, 2010).

However, apart from the cooperative and its production activities, there is no particular action or project for TEK conservation in the research site. It is also worth noting that there is not any governmental concern and recognition of TEK and indigenous rights in the research site (Ao, Aote, Da, Wang, interviews, 2010; participant observation, 2010). The policy and efforts to support cooperative organisations primarily target improving economic well-being of the rural populations (see Chapter 5, section 1.4 for more details of the law).

\footnotetext{
66 The association is a town-based NGO with an aim to provide information services to rural cooperative organisations.
} 


\subsection{Action analysis \& Assessment}

From this case study, we can see that, even though the initial target of the cooperative was not TEK conservation, the cooperative has enabled participating families to partially revitalise their traditional herding rotations and communal production system. Through these practices, the traditional community cohesion has been strengthened, and traditional community leadership has been partially revitalised. As a result, the cooperative has attracted more families to join. As the cooperative leaders predicted, with the participation of more families even all families in the village, there is a great potential that the traditional four-season herding rotation and communal working relationships amongst the community members can be revitalised.

These activities can be categorised as community-based TEK conservation activities (the revitalisation of herding rotations) and indigenous capacity building according to the TEK conservation action typology. The indigenous capacity building included all three sub-categories - institutional development (the cooperative is self-organisation), alliance and partnership development (partnership with the local NGO, and network with other two cooperatives in Inner Mongolia ), and indigenous financing (cooperative forums in which participating cooperatives shared information on fund sources and experience with funding application and fund management). Behind these actions, the governmental policy and legislation have played an important role in triggering and supporting the development of the cooperative.

It is a useful exercise to link the conservation actions back to the threats to see which threats have been addressed by conservation actions, and then obtain an understanding of the conservation efficiency. Below is a summary of the threats, actions and their links in the Bayin case study. 
Table 6.1 A Summary of TEK Threats, Conservation Actions and their Links in the Bayin Case Study

\begin{tabular}{|c|c|}
\hline Threats & Current Conservation Actions \\
\hline \multicolumn{2}{|l|}{ Direct threats } \\
\hline 1. Change of traditional livelihood practices & $\begin{array}{l}\text { Community-based TEK conservation } \\
\text { activities (the revitalisation of herding } \\
\text { rotations); } \\
\text { Policy and legislation (the Cooperative Law) }\end{array}$ \\
\hline 2. Loss of traditional rights & Not addressed \\
\hline 3. Loss of traditional institutions & $\begin{array}{l}\text { Indigenous capacity building; } \\
\text { Policy and legislation (the Cooperative Law) }\end{array}$ \\
\hline $\begin{array}{l}\text { 4. Change of environment and natural } \\
\text { resources }\end{array}$ & $\begin{array}{l}\text { Community-based TEK } \\
\text { conservation } \\
\text { activities (at a limited level, the } \\
\text { environmental benefits of revitalising } \\
\text { herding rotations is long term) }\end{array}$ \\
\hline 5. Loss of pathways of TEK transmission & Not addressed \\
\hline \multicolumn{2}{|l|}{ Underlying threats } \\
\hline 1. Government policy and legislation & Not addressed \\
\hline 2. Contact with other cultural groups & Not addressed \\
\hline 3. Economic development pressure & Not addressed \\
\hline
\end{tabular}

Table 6.1 illustrates that the current conservation actions at the community level addressed certain direct threats at certain levels (i.e. change of traditional livelihood practices, loss of traditional institutions, and natural resource degradation). At the government level, the Cooperative Law and its supporting measures provided a chance for traditional practices and traditional institutions to be revitalised. However, dealing with all underlying threats (i.e. TEK-unfriendly government policy and legislation, contact with other cultural groups and economic development pressure) is beyond the community capacity; yet has not been targeted by upper institutions (i.e. local and central government) at all. 
According to the recommendations for TEK conservation design, three principles were considered essential to achieve effective TEK conservation: 1) an appropriate policy and legislative framework at multiple levels; 2) genuine engagement of indigenous people in wider social-economic-political decision-making process; and 3) effective indigenous capacity building (for detailed discussion, please see Chapter 4, section 3.1). Two principles are partially achieved in this case study:

\section{1) Policy and legislative support to cooperative development at multiple levels. In}

this case study, the national legislation (the Cooperative Law) and its supporting policies have been implemented by the authorities at national, regional and local levels. These actions have positively fostered the establishment of the community cooperative and its activities. Although historically Chinese policy was very detrimental to indigenous institutions, recent policy shifts have provided some (unintentional) support for revitalising indigenous community practices. As I discussed previously, policy and legislation is a double-edged sword. On one hand, TEK-supporting policy and legislation may effectively protect and conserve TEK (e.g. Wavey, 1993; Zent, 1999); on the other hand, TEK-unfriendly policy and legislation may effectively cause TEK degradation (e.g. Wang, 2009; Xu and Melick, 2007). Thus, the awareness of the significance of government policy and legislation on TEK and indigenous populations should be raised within the authority system. Scale is also a critical factor in determining possible TEK trajectories. Because of the complex, multi-scale nature of the social-ecological system in which Inner Mongolian TEK is embedded, authorities at every level share the responsibilities of recognising and protecting TEK and indigenous rights (Brondizio et al., 2009; Ostrom, 2009). Cross-scale institutional cooperation is crucial for achieving effective conservation outcomes (Anderies et al., 2006; Lemos and Agrawal, 2006; Nelson et al., 2010). Any missing effort from any scale will potentially lead to the failure of the conservation efforts overall.

2) Indigenous capacity building. The cooperative itself is self-organised; it was initiated and managed by the community with the aim of promoting traditional practices and enhancing economic well-being. It also encompassed a partnership with an external NGO and a network with other cooperative organisations. The cooperative showed its concern and efforts in developing its financial capacity. These actions 
demonstrate that the capacity building in Bayin community has achieved a certain degree of success. Though, there is still great potential for capacity building to be advanced. Community capacity is a comprehensive set of knowledge, resources, skills, institutions and actions (Bruce, 2003; Simpson et al., 2003). According to Simpson et al. (2003), the need and awareness of capacity building from inside of the community is a driving force of true engagement. The success of capacity building depends on knowledge and genuine skill acquisition - processes that take considerable time and application, and thus require on-going external support. However, in most of cases, such support is insufficient (Baker and Teaser-Polk, 1998; Casswell, 2001). The policies and actions from the authority system with an aim of community empowerment have to be sincere and genuine (Murdoch and Abram, 1998).

Despite some achievements of applying the above two principles, there is a severe deficiency of genuine engagement of indigenous people in socio-economic-political decision making processes in the research area and in China generally. For example, in the Bayin case, community members are only able to practice their traditional knowledge and skills within the cooperative framework and these rights do not extend beyond. This is because the indigenous rights have not been genuinely recognised by the government authorities (Ao, 2003, 2005; Tang and Gavin, 2010). Thus, the cooperative may achieve certain success in terms of revitalising traditional herding practices and enhancing the community's economic well-being; however, for the long term well-being of indigenous Mongolian culture, traditions and rights, a strong policy and legislative framework and wider socio-political support that allow effective participation of indigenous populations in decision-making are missing.

As discussed previously, the concern and pursuit of community engagement, as a part of governance decentralisation, has been an international trend for several decades. It is believed that the decentralisation reforms can achieve participatory development, greater administrative efficiency, benefit local empowerment and increase local accountability. It may also be useful in poverty reduction and resource sustainability (Colfer and Capistrano, 2005; Lemos and Agrawal, 2006; Ribot, 2002). However, as many scholars point out, there is a significant gap between the theory and the practice of this decentralisation process (e.g. Bene and Neiland, 2006; Berkes, 2010; Larson and 
Soto, 2008; Ribot et al., 2006) (see Table 6.2). For example, as Ribot (2002) points out, most decentralisation reforms are characterised by insufficient transfer of power to local institutions. In addition, local jurisdictions have not been receiving the resources commensurate with their responsibilities (Pomeroy and Berkes, 1997).

Table 6.2 The Gap between the Theory and the Practice of Decentralisation

\begin{tabular}{|c|c|}
\hline Theory of Decentralisation & Practice of Decentralisation \\
\hline $\begin{array}{l}\text { Participatory development and greater } \\
\text { efficiency for local priorities }\end{array}$ & $\begin{array}{l}\text { Local jurisdictions not receiving powers or } \\
\text { resources }\end{array}$ \\
\hline $\begin{array}{l}\text { Increased voice for local communities, } \\
\text { empowerment and democratisation }\end{array}$ & $\begin{array}{l}\text { Elite capture of resources, as the powerful } \\
\text { local take advantages of uncertainty }\end{array}$ \\
\hline $\begin{array}{l}\text { Poverty reduction through equitable access } \\
\text { to resources }\end{array}$ & $\begin{array}{l}\text { Marginalisation of the extremely poor and the } \\
\text { disadvantaged groups }\end{array}$ \\
\hline $\begin{array}{l}\text { Greater accountability in } \\
\text { governments }\end{array}$ & $\begin{array}{l}\text { Lack of representativeness of decentralised } \\
\text { bodies }\end{array}$ \\
\hline $\begin{array}{l}\text { Tailoring resource management objectives } \\
\text { to local context }\end{array}$ & $\begin{array}{l}\text { Fragmentation management responsibility for } \\
\text { ecosystems }\end{array}$ \\
\hline 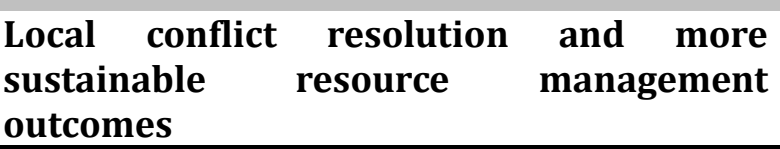 & $\begin{array}{l}\text { Creating more local conflicts and social } \\
\text { tensions, some leading to resource overuse }\end{array}$ \\
\hline
\end{tabular}

Sources: Berkes, 2010: 492

Based on works of Bene and Neiland (2006), Colfer and Capistrano (2005), Larson and Soto (2008) and Ribot (2002)

Successful decentralisation requires that the practice is consistent with the theory. In order to achieve genuine community engagement and good governance, the willingness and actual actions of power devolution from the authority system and efficient communication across different levels of the institutions are equally important (Berkes, 2010). 


\section{RECOMMENDATIONS FOR FURTHER CONSERVATION}

\section{ACTIONS}

There is a great potential for more TEK conservation to be undertaken in this research site. The TEK action typology points out potential actions beyond what have been undertaken in the research area. Based on the local context and wider social-economicpolitical environment in China, and informed by the analysis of the current actions and the design principles for effective TEK conservation, I suggest three potential actions below:

1) Policy and legislative support at more comprehensive levels. The Cooperative Law is a good example of how legislation and policy can aid TEK protection. Nonetheless, given the history of government intervention and the negative impacts of past government policy on traditional institutions in the region, a single piece of legislation is far from sufficient for achieving comprehensive TEK conservation. Policy and legislative support to indigenous communities and protection to indigenous rights at multiple levels is required. The government authorities at all levels should recognise the importance of TEK and indigenous rights protection, and provide active efforts.

A framework of indigenous or minority's rights protection theoretically exists in China. According to the Constitution of China (the Central Government of China, 1982: Chapter 1, Article 4), "The State protects the lawful rights of various ethnic minority groups... All minority groups have the freedom to use and develop their own language, to maintain or reform their own culture and customs". In Chapter 3, Article 119, the Constitution states that, "The minority autonomous authorities are responsible for selfdetermination of local education, science, culture, health, and sports development, the protection of the traditional cultural heritage, and the development of the minority's traditional culture". Based on these principles of the Constitution, the state government also developed the Chinese Ethnic Minority Autonomy Law (the Central government of China, 1984). It further defines the rights of self-determination, the use of indigenous language, maintaining cultural practices and religions of all ethnic minorities in China. 
The Chinese government also signed the Convention of Biological Diversity during the UNCED on June 11, 1992 and became one of the earliest countries to ratify the CBD on January 5, 1993 (CBD, 2011). This indicates the recognition and agreement of protecting indigenous knowledge and practices, as the CBD 8(j) notes that, each Contracting Party shall, as far as possible and as appropriate:

Subject to its national legislation, respect, preserve and maintain knowledge, innovations and practices of indigenous and local communities embodying traditional lifestyles relevant for the conservation and sustainable use of biological diversity and promote their wider application with the approval and involvement of the holders of such knowledge, innovations and practices and encourage the equitable sharing of the benefits arising from the utilization of such knowledge, innovations and practices.

However, these promises have often been undermined in reality by the dominant economic development policy in China (Da and Zheng, 2010; Wang, 2009). The Bayin case study presents an example here. The protection of TEK and indigenous rights has been compromised by local economic development priorities, such as mining and urbanisation. How to truly achieve these promises is a huge challenge to both indigenous populations and the government authorities in China. Not in China alone, this challenge has been commonly reported across the globe. To date, a policy and legal framework of TEK protection at the international level has been built (e.g. the Agenda 21, the CBD, the Declaration on the Rights of Indigenous People, and the Rio Declaration). However, how to transfer these policy and legislations into actions at local, regional and national levels remains a major challenge to all indigenous populations and authorities.

Based on the specific social-political environment in China, policy and legislative support at multiple levels may be the only effective way to deal with both direct (especially the loss of traditional rights) and all underlying threats in the research area and wider areas of China. As the Bayin case demonstrates, government policy and legislation have been the major underlying cause of TEK degradation in the research region. The current centralised power structure in China makes it is impossible for any other institutions (e.g. communities and NGOs) but the government to deal with these 
underlying threats. Therefore, the responsibility of decentralising the power and providing policy and legislative support towards indigenous populations lies with the Chinese government.

2) Community-based TEK conservation activities. So far, in the research site, the main focus of the cooperative participants is economic well-being and community cohesion. Wider domains of TEK have not been emphasised enough. Nonetheless, the existence of the cooperative has created a platform that might be used for developing other TEK-focused community activities. For example, traditional lifeways programmes can stimulate the interests in maintaining traditional culture and using traditional practices. The programmes can be developed based on the community interest preference, such as community cultural festivals, which may include folk music shows, horse riding races, Mongolian wrestling, traditional rituals and ceremony showcases; elder and youth days, which may include trace reading training, horse riding training and grassland vegetation training. These activities target the loss of pathways of TEK transmission (direct threat) that has not been dealt with so far (see Table 6.1). These activities may function more effectively through a community alliance, in which several neighbouring communities participate.

Part one of this research collected useful examples from other countries and regions which may be inspirational here. For instance, in Pikangikum, Ontario of Canada, the First Nations have carried out a programme named "culture days" for years. The programme permits indigenous children to be absent from schools in order to participate in hunting and other traditional seasonal livelihood activities. These children therefore are able to learn traditional knowledge and practices from their communities (Miller, pers. comm., 2011). Also in Canada, the Winnipeg of Manitoba has a programme called "Elder camps and Youth Challenge", which has been organised by the First Nations since 2007. Within Elder camps, the community youngsters have an opportunity to learn from their elders. They eat traditional food, and go for plant walks with the elders. During the plant walks, youngsters learn traditional knowledge of plants and plant use from the elders. Youth challenge organises canoe and camping races for youngsters. They are not allowed to bring any supplies to the camps. Under the supervision of elders, they learn how to use natural materials to make canoes or to meet camping needs (surv. 
resp., 2010). The significance of these activities is to enable indigenous youngsters have more contact with their community and their elders, and to learn and appreciate their traditional lifestyle and practices. Within these activities, the TEK transmission has been strengthened.

3) Educational means. Education and awareness is another powerful means that can be applied in this case study. It may address the loss of pathways of TEK transmission (direct threat) and contact with other cultural groups (underlying threat) by promoting traditional culture and knowledge and increasing appreciation of TEK among indigenous Mongolian population and the general public. In the research area, even though the compulsory education system is constructed on the modern Westernised ideology, the ethnic minority's autonomy has been applied on the education sector at a certain level. There are two types of primary and secondary schools in Xiwu city Mandarin speaking or Mongolian speaking. The school students have the right to choose according to their own preference. In the Mongolian speaking schools, the contents of curriculum include more TEK than in the Mandarin schools. Though, many parents decide to send their children to Mandarin schools, because the tertiary education system and urban jobs require Mandarin skills. The attendance at Mongolian schools could be promoted and encouraged.

The application of different means of educational tools depends on the community capacity. In the Bayin case, customary education, such as community schools, faces many barriers due to the strong government enforcement of the compulsory education policy. The government also generally lacks interest or willingness to embrace alternative educational models (Zhao, interview, 2010). However, the application of informal learning may fill this gap, such as traditional culture showcases and a community museum. Informal learning can be easily combined with the communitybased TEK conservation activities. In this sense, the indigenous community from the Chitwan National Park of Nepal offers a comparable example. The community established their own museum in 1998. The museum organises exhibitions of traditional culture and livelihood activities. It provides a physical space for community members and the wider public, especially the youth to learn indigenous traditions, culture and traditional knowledge of cultural important plants and animals (surv. resp., 
2010). The museum also holds performances of traditional dance, folk music and traditional costume showcase (for more information, see http://chitwannationalpark. net). Through these activities, community members, especially the youth have developed their interest in and proud of their traditional culture and practices.

In summary, multiple actions at multiple levels, with multiple actors involved are required for comprehensive TEK conservation. For the community level, utilising the foundation of the cooperative organisation, and developing community-based TEK conservation activities (e.g. traditional lifeways programmes) is a practical approach. Informal TEK learning can also be organised within this framework. Meanwhile, the community capacity building, especially the institutional development should remain a major concern. The NGO's continuous support to the community is helpful. At the local and regional levels, the governmental efforts can focus on five aspects: 1) the supporting policies to the cooperative organisations should be constant and consistent; 2) to grant official recognition of TEK and indigenous practices; 3 ) to grant more support towards pastoralism rather than urbanisation and industrialisation; 4) should affect the educational factors to recognise and include more TEK in the curriculum; and 5) to provide more financial support to pastoralism and herders' cooperative organisations. At the national level, the legislation and policies regarding indigenous rights and TEK, such as the Ethnic Minority Law, the Constitutions and the CBD, need corresponding action strategies that will allow for more meaningful support of TEK revitalisation at lower levels.

\section{CONCLUSION}

This chapter has provided a complete model of how to apply the TEK threat and action typologies into real-world situations. Through the assessment of all threats in the case study, the threat typology provided a useful tool for identifying and analysing TEK threats. I found the categories and sub-categories are comprehensive, and easy to use. The distinction between direct and underlying threats in the threat typology was helpful for obtaining the understanding of the causal web of TEK degradation within the research context. It then enabled me to draw an overview of the multi-level threat 
system that Mongolian TEK faces in the research region, and informed the design of potential conservation actions.

Through the analysis of the current conservation actions, I found the mapping of realworld actions into the conservation typology was easy and straightforward. More importantly, the conservation typology provided a wide spectrum of potential conservation options to choose from. This is because the development of the typology was based on a wide collection of cases ( $\mathrm{n}=137$, from more than 48 countries and regions), and data from a wide range of literature $(n=152)$. The typology therefore presents a comprehensive set of solutions that assemble collective experience and wisdom of TEK holders, practitioners and scientists from over the globe. This exercise demonstrates that the TEK conservation typology could be used in a similar way for TEK holders and practitioners in wider regions in their TEK conservation design and planning.

Below is a summary of TEK threats and conservation actions identified in the case study analysed here.

Table 6.3 A Summary of TEK Threats, Conservation Actions and Recommendations for Further Conservation Actions in the Bayin Case Study

\begin{tabular}{|c|l|}
\hline \multicolumn{1}{|c|}{ Direct threats } & $\begin{array}{l}\text { 1. Change of traditional livelihood practices } \\
\text { 2. Loss of traditional rights } \\
\text { 3. Loss of traditional institutions } \\
\text { 4. Change of environment and natural resources } \\
\text { 5. Loss of pathways of TEK transmission }\end{array}$ \\
\hline Underlying threats & $\begin{array}{l}\text { 1. Government policy and legislation } \\
\text { 2. Contact with other cultural groups } \\
\text { 3. Economic development pressure }\end{array}$ \\
\hline TEK conservation actions & $\begin{array}{l}\text { 1. Indigenous capacity building } \\
\text { 2. Policy and legislation } \\
\text { 3. Community-based TEK conservation activities }\end{array}$ \\
\hline Recommendations for further & $\begin{array}{l}\text { 1. Policy and legislative support at a more } \\
\text { comprehensive level } \\
\text { 2. Community-based TEK conservation activities } \\
\text { 3. Educational means }\end{array}$ \\
\hline
\end{tabular}


In a short summary, the Bayin community faces a complex web of threats to their TEK, including five direct threats and three underlying threats. To deal with TEK degradation, the community initiated a community-based TEK conservation project (the cooperative activities), in which community capacity building was able to developed, and policy and legislative support was granted. In turn, these conservation actions addressed certain direct threats at certain levels (change of traditional livelihood practices, loss of traditional institutions and natural resource degradation). The loss of pathways of TEK transmission and loss of traditional rights (direct threats) have not been addressed. Dealing with underlying threats is beyond the community capacity; yet has not been targeted by the upper institutions (the local and central government). Thus, my recommendations for the further actions emphasised on policy and legislative support from the authorities (targeting all underlying threats and two direct threats - loss of traditional rights and loss of pathways of TEK transmission), community-based TEK conservation, and educational means (targeting the loss of pathways of TEK transmission and further addressing other direct threats).

One case study is an extremely limited number. A systematic test of a classification system should be a long-term and on-going process. For example, the IUCN-CMP classifications of direct threats to biodiversity and classification of biodiversity conservation actions have been developed since the early 2000s (CMP, 2004, 2005; CMP-IUCN, 2007). The classifications have been tested with hundreds of projects and thousands of species around the world over years (Salafsky et al., 2008). Even so, the IUCN-CMP is still reviewing the classification system, and it is still open for adjustments (IUCN, 2005, 2006). This illustrates that the development of such a classification system is a long-term, adaptive process, in which continuous testing, feedback and adapting are essential. The testing and modifying process of the TEK typologies will also be a longterm, on-going and adaptive process. This chapter may be considered as an initial effort that can inspire and promote much wider testing exercises and modification to the typologies into a completely applicable standard.

Author's Note: One of the important functions of TEK typologies is enabling the comparison across projects and across regions. In this chapter, I provided an analysis based on one case study; but it would be more useful to present comparison between 
different studies. I have carried out the same analysis to another case study in western Mongolia which I conducted between 2006 and 2007, and compared the results between these two case studies. Due to the word limit of this thesis, I will not present the details here; for a brief summary of the comparison, please see Appendix 9. 


\section{CHAPTER SEVEN}

\section{CONCLUSION AND RECOMMENDATIONS}

The aims of this research were to explore global patterns in the status of TEK, the threats that TEK faces, and the available TEK conservation actions, and to understand how institutional linkages between government and community affect TEK and its conservation in Inner Mongolia of China. By applying a survey $(n=137)$ and follow-up interviews $(n=46)$, a comprehensive literature review $(n=152)$ and one in-depth case study, I developed a standard classification system of TEK threats and conservation actions, which will be useful for analysis and decision-making in real-world case situations. In Chapter One, I addressed key literature gaps in the TEK research field: the lack of a review of 1) global patterns in the status of TEK; 2) threats to TEK; and 3) TEK conservation options; 4) the lack of standard classification system of TEK threats and conservation actions; 5) the status of TEK and conservation of TEK in Inner Mongolia China is largely unknown to the outside world; and 6) the limited understanding of the role of government policy on TEK trajectories in Inner Mongolia. Through Chapter Two to Chapter Six, the thesis presented a wide range of findings and made its contributions to fill these literature gaps.

Below, I will briefly review the key findings under each research objective; then summarise the main emerging themes that developed from this research. I will close the thesis by discussing limitations of this research and recommendations for future research, and making some brief concluding remarks.

\section{KEY FINDINGS}

\subsection{Objective One - To explore any global pattern in TEK status (Chapter 3)}

The overall trends of TEK change explored from the survey and the literature present a similar pattern - the vast majority (87\% of the survey respondents and $89 \%$ of TEK studies in the literature) noted a pattern of TEK loss, and this pattern held across all regions examined. The increase ( $7 \%$ of the survey respondents and $3 \%$ in the literature) 
and no change ( $2 \%$ in both the survey and the literature) of TEK were noted at minor rates.

\subsection{Objective Two - To identify the threats faced by TEK globally and to develop a TEK threat typology (Chapter 3)}

In Chapter Three, the research synthesised the multiple causes of TEK degradation identified by the survey respondents and in the literature. I then organised these TEK threats into a classification system. Threats were categorised into six direct threats and ten underlying threats. While direct threats refer to human activities and processes that directly cause the destruction, decline, and/or degradation of TEK conservation target; underlying threats are the causal factors of direct threats, which usually include cultural, economic, political and institutional factors.

\section{The direct threats include:}

1) Loss of pathways of TEK transmission - Complete or partial loss of the TEK transmission processes between individuals and generations; within a community and/or across communities.

2) Change of traditional livelihood practices - Reduced needs and practices of, or abandonment of traditional livelihood, which often involves a transition from traditional subsistence livelihood to non-traditional livelihood activities, such as engaging with industrial and trading activities.

3) Change of traditional religion and belief - Loss or change of the traditional religion and beliefs in the importance of nature and the local environment.

4) Change of environment and natural resources - Change of physical environment due to relocation, and/or degradation or depletion of natural resources.

5) Loss of traditional rights - Complete or partial loss of traditional land, loss of resource or resource rights, and loss of traditional access to natural resources.

6) Loss of traditional institutions - Complete or partial loss, or change in traditional social organisations, customary norms and traditional rules of resource use and management.

Among these six categories, the most frequently ( $>30 \%$ in both the survey and the literature review) noted direct threats were loss of pathways of TEK transmission, and 
the change of traditional livelihood practices.

The underlying threats include:

1) Government policy and legislation - Policy and legislation made by dominant, nonindigenous societies that devalue and/or suppress indigenous groups and their cultures. 2) Contact with other cultural groups - Can be caused deliberately by non-indigenous groups or voluntarily by indigenous groups.

3) Influence of outside market - Including the availability of modern goods, access to market and possibility of engaging with trading activities.

4) Colonisation - The establishment, maintenance, acquisition and expansion of colonies in one territory by people from another territory.

5) Relocation-Enforced or voluntarily movement of indigenous peoples from their traditional lands.

6) Marginalisation by dominant societies - Including social, economic and political marginalisation, often leading to reduced self-esteem of indigenous populations.

7) War and military occupation of indigenous peoples' land and/or resources.

8) Indigenous population decline - Including natural demographic decline or humaninduced incident, such as genocide.

9) Migration - Including indigenous emigration and non-indigenous immigration.

10) Economic development pressure - Sustained and concerted actions that promote the standard of living and economic health of a specific area, usually driven by the dominant market economy.

The frequencies of these ten categories addressed by the survey respondents were relatively even; the most frequently listed underlying threats were contact with other cultural groups (20\%), migration (17\%) and influence of outside market (16\%). The most frequently listed underlying threat in the literature was influence of outside market $(31 \%)$

The TEK threats cover a broad range of issues, including political, social, cultural, economic, educational and ecological. All these threats act simultaneously in a complex manner. Chapter Three also provided detailed analysis of the pathways of TEK degradation under each direct threat. The TEK threat typology can be used 1) by 
indigenous communities and their organisations to identify and target the problems that threaten their knowledge and practices, and enable them to communicate across communities and share information; 2) by the academic and scientific community to enhance cross-project and cross-region communication and analysis of broad scale patterns, and to use in constructing common TEK databases; 3) by non-governmental organisations to examine TEK threats in a location and prioritise resources and funds for organisational planning; 4) by government agencies to evaluate TEK status in the region or country and identify the major TEK threats, as well as to produce and implement conservation policies and measures; and 5) by international policy organisations to draw a global picture of TEK status and TEK threats, to identify and prioritise the most important TEK conservation hotspots where international funds should be allocated. These organisations will also be enabled by the typology to advise better on the TEK conservation efforts of national governments.

\subsection{Objective Three - To explore the TEK conservation actions and develop a TEK conservation action typology (Chapter 4)}

Following a similar procedure, Chapter Four explored multiple TEK conservation options, including both applied actions and theoretical options suggested in the literature. It then developed a classification system of TEK conservation options which includes five categories:

1) Indigenous capacity building - A long-term, on-going process in which indigenous communities build or improve their collective resources and skills to conserve and/or revitalise their traditional culture and lifestyles.

2) Community-based TEK conservation activities - The conservation actions undertaken in the indigenous sites, usually with the participation of indigenous people with a target of conserving and revitalising TEK.

3) Education and awareness - The conservation and promotion of TEK through educational systems and public awareness raising.

4) Policy and legislative support - The establishment and implementation of policy and legislation that protect indigenous rights, support and encourage traditional culture and practices.

5) Research and documentation - The study and recording of TEK by TEK holders, 
outside institutions, or their joint efforts.

According to the survey results, the majority $(82 \%, n=112)$ of the survey respondents claimed that TEK conservation actions had been undertaken in their locations. Within these actions, the three most frequently used options are research and documentation (39\%), community-based TEK conservation activities (25\%), and education and awareness (16\%).

\subsection{Objective Four - To explore the triggers and barriers to TEK conservation (Chapter 4)}

In Chapter Four, I also examined the outcomes of the conservation actions. Due to the lack of relevant data in the literature, this examination was based on the survey results. Among the respondents who claimed conservation actions had taken place in their locations $(n=112)$, the vast majority $(82 \%)$ claimed the actions had some success. The key factors related to success included: 1) the involvement of local community in the conservation projects; 2) external support to local-based projects; and 3) governmental recognition and support to TEK and TEK conservation projects.

Meanwhile, five barriers to TEK conservation success were identified: 1) the competing interests or cultural influence of non-indigenous, dominant societies; 2) the lack of governmental recognition and support; 3) the lack of financial resources and other supporting capacity; 4) outside agencies dominating projects leaving no space for community initiatives and participation; and 5) wider social, economic and political stress.

Based on the triggers and barriers identified from the survey responses, theoretical discussions in the literature and my own analysis, this research then summarised three design principles for achieving effective TEK conservation:

1) An appropriate legislative framework at multiple levels is essential.

2) Genuine engagement of indigenous people in wider social-economic-political decision-making process is highly desirable.

3) Effective indigenous capacity building is vital to TEK and indigenous survival and revival. 
In sum, a successful TEK conservation regime should incorporate internal motivation and capacity building from within the indigenous population, and external recognition and support from wider realms. As direct and underlying threats interact in a complex web of causality which shapes TEK change over time, TEK conservation will only have a chance at success when both direct and underlying threats are addressed.

\subsection{Objective Five - To examine the historical government policies in China and their effects on traditional institutions and traditional herding practices in Inner Mongolia (Chapter 5)}

By applying in-depth semi-structured interviews $(n=91)$ and focus group discussions $(n=5,64$ participants), this case study of Bayin Wenduri village in Inner Mongolia concluded that the traditional institutions and traditional practices of Mongolian herders had been adversely affected by governmental policies from the 1960s through the early 2000s. This includes the enforced transformation of land and resource ownership from collectively-owned to state-owned in the 1960-1970s; the enforced settlement and privatisation of land and resource user rights in the 1980-1990s; and the continuous pressure to pastoralism from industrialisation and urbanisation in the 2000s.

The study found that, although the local knowledge of traditional resource use and traditional institutions still exist in the research site, the active use of such knowledge had been curtailed for at least four decades. As such, the local TEK system has reached a threshold point in which permanent loss of the knowledge will be inevitable if no immediate conservation actions are undertaken (Groffman et al., 2006; Huggett, 2005; Wang, 2009).

\subsection{Objective Six - To examine the development and impact of community cooperatives for traditional herding practices in Inner Mongolia (Chapter 5)}

The Inner Mongolia case study found that the emergence of cooperative organisations has been a grassroots level movement, and the central government positively responded 
to the movement. The establishment and implementation of the Cooperative Law and its supporting policies provided positive support to the development of cooperatives at the community level.

Within the cooperatives practices, three or four-season rotation of pasture lands was revitalised. Participating families linked their private pasture lands and worked together as a collective unit with specific job divisions (e.g. herding, hay nursery and animal nursery units). The management processes enshrined traditional rules of resource distribution, job division and benefit allocation. The elders played an important role in cooperative decision-making, and contributed their knowledge of resource management and special skills at conflict solving into the cooperative management. In the meantime, the younger leaders deal more with external issues, such as marketing, crossorganisational exchange and dealing with the government. This model presents an adaptive development of the community institution - a combination of traditional practices (collective decision making and elder's leadership) and new resource management approaches (young leadership in dealing with external issues).

\subsection{Objective Seven - To examine the triggers and barriers to the cooperative organisations aimed at the revitalisation of traditional herding practices in Inner Mongolia (Chapter 5)}

The triggers and barriers for revitalising cooperative management of pasture lands came from both inside the community and external influences. The need and willingness for collective efforts from the community members formed the initial push of the cooperative movement (from the late 1990s). The central government responded to the movement by establishing the Cooperative Law (2007) and supporting measures. The local government implemented the supporting measures that required by the law. Finally, with the assistance of a local NGO, the community cooperative organisation was formed and developed. This case reflects that external support for community-based conservation (both TEK and environmental conservation) actions through institutional linkages functions as a critical factor that enables the success of these community-based approaches to the revitalisation of traditional resource management practices. 
In terms of the barriers to revitalising traditional resource management practices in the Inner Mongolian case, a core issue is the vaguely defined land tenure (Wang, 2007). Without appropriate rights to the land and resources, the foundation of cooperative activities is weak. To date, the state still owns the land; herders are only holding "exclusive user rights" to the land and resources (Ao, 2005). This means once the government changes its policy regarding land use, herders may have to abolish their herding practices. Disturbance also comes from conflicting governmental policies. On one hand, the government is promoting cooperative movement; on the other hand, the priority of the local government is industrial development and economic growth (The Bureau of Statistics China, 2010), which often compromises pastoralism in favour of extractive industries. Lastly, the unwise use of the grassland resources over the last 60 years has led to severe land degradation. The local ecosystem has reached an ecological threshold point (Ao, 2003). The degraded state of the resource creates a physical difficulty for future herding activities.

The analysis of triggers and barriers reflects the social-ecological system in which this community case study was embedded. The future of the cooperative movement and the community well-being is determined by an inter-connected web of central government policies, local government priorities, community capacity, external organisations' performance and local ecological conditions.

\subsection{Objective Eight - To explore the possibilities of revitalising TEK through the cooperative movement (Chapter 5)}

There is great potential for Mongolian TEK to be revitalised under the current cooperative movement. Two major elements of TEK have been the focus of revitalisation efforts within the cooperative activities. Most significantly, the herding rotation (a small scale, three-season rotation in Bayin gacha, and a village-wide, four-season rotation in Hulun gacha) has been revitalised. This is a critical achievement for Mongolian TEK revitalisation, especially after 30-40 years of complete abandonment of rotations. The second most important element of TEK revitalisation was the emphasis on traditional community institutions and community resource governance. During the cooperative process of "joining-pasture and working-together", the participating community members resumed their traditional collaborative working relationships. Many 
community informants claimed that the bond amongst community members has been strengthened since the cooperative practices. Within the cooperative frame, the traditional community elders' leadership was also revitalised. Elders were traditionally regarded as having noble values, abundant knowledge and authoritative nature within the community (Da, 2008). The belief in elders' knowledge and judgement, and willingness to accept elders' leadership is still common within the community. In both villages, respected elders were invited when the cooperatives were initiated, and took a leading role in decision making.

This study demonstrated that the development of cooperative organisations created opportunities for community autonomy regarding land use and resource management. The cooperative activities empowered the community to play a role in cross-scale resource governance. However, the cooperative movement is still at an early stage in China (World Bank, 2006; Young et al., 2006), and its long-term effects, especially in terms of revitalising TEK and empowering the community, still require continuous monitoring and further research.

\subsection{Objective Nine - To test the applicability of the TEK typologies developed from part one of this research (Chapter 6)}

Chapter Six provided an example of how to apply the TEK threat and action typologies into real-world situations. I applied the typologies to the Inner Mongolia case study to analyse the causes of TEK degradation and corresponding conservation actions within the local context. The analysis demonstrated that the threat typology can provide a powerful tool for identifying TEK threats, and the action typology can provide a spectrum of conservation options to choose from. Through the case analysis exercise, I found the typologies are reasonably applicable and useful for the analysis. It was relatively easy and straightforward to find matching categories and sub-categories from the typologies into the case situation. This chapter functions as a trial of the typologies; an initial effort to inspire and promote much wider testing and application exercises. 


\section{EMERGING THEMES}

\subsection{Applying TEK typologies in a social-ecological system (SES)}

One of the core messages produced from this research is that dealing with TEK degradation and implementing TEK conservation has to take a systematic approach and a more holistic view of the complex social-ecological systems in which TEK is situated. By identifying the complex, dynamic interactions between natural and social processes, SES theory provides an intellectual foundation for this research. As Ostrom et al. (2009) point out, within a SES subsystems (i.e. ecological, social, economic and political settings) affect each other and are constantly interacting. This theory shares a significant common ground with indigenous worldviews and their approaches of resource management (Berkes and Folke, 1998; Ostrom, 2009). In recent years, the increasing application of SES theory has transformed environmental management in many ways, such as the call for more collaborative management regimes (e.g. Bidwell and Ryan, 2006; Gavin et al., 2007; Koontz and Thomas, 2006), more institutional linkages across scales (e.g. Agrawal, 2002b; Anderies et al., 2004; Ostrom, 2009), and for adaptive management approaches (e.g. Berkes et al., 2000; Folke et al., 1998). Several key attributes of the SES theory have significant application in this research.

First, the complex interdependence between natural and social systems highlights that the study and conservation of TEK needs to take an interdisciplinary approach, in which principles and techniques from both ecological and social sciences are integrated (Anderies et al., 2004; Liu et al., 2007). For example, in the TEK threat typology, the categories and sub-categories cover the issues of language, education, migration, market influence, law and policies, religion, indigenous rights and institutions, and so on. In all instances, there is no single discipline or individual organisation that is able to address all these components. In turn, a multi-disciplinary and systematic approach has to be taken to adequately address the complexity of the challenges TEK faces in most contexts.

Second, the scales across space, time and organisational units within an SES require that the analysis of TEK threats and design and implementation of conservation actions have to be multi-scalar. For example, for the designing of TEK conservation action, a wide range of possibilities are provided in the conservation typology, including capacity 
building and TEK conservation activities at the community level, alliance and networks at regional, national even international levels, and policy and legislative support at national and international levels. As such, this research emphasises that the planning and implementation of TEK conservation is a systematic matter, in which linkages across scales play a crucial importance. It also highlights that since the TEK threats come from multiple levels (including both direct and underlying threats), the conservation action has to be multi-scalar that addresses both direct and underlying threats.

Lastly, this research indicates that TEK systems are subject to the dynamic, complex and uncertain nature of SES. It then requires that TEK conservation has to take explicitly adaptive management approaches. In such approaches, uncertainty of both social and ecological systems is recognised, and flexibility of decision making based on the local conditions and continuous monitoring can be achieved. Findings from the Inner Mongolia case study highlight the importance of thresholds and resilience in this SES. The study suggests that, even though knowledge of traditional land use and institutions still exists to a certain extent (mainly held by elders), that knowledge has been in very limited use over the last five decades. This TEK system, then has reached a threshold point, in which permanent loss will be inevitable if no immediate conservation action is undertaken and the elders which hold the knowledge pass away (Wang, 2007, 2009). This case is unlikely to be alone. This finding reflects a similar trend that has been noted in the critical change of TEK in many locations around the globe (e.g. Inglis, 1993; Berkes, 2008; Liu et al., 2007). As many scholars suggest, the occurrence of threshold points signifies the urgent need for immediate conservation actions (e.g. Huggett, 2005; Walker and Meyes, 2004; Wang, 2009).

\subsection{Institutional linkages in TEK conservation}

There is also a need to design and support TEK conservation via cross-scale institutional linkages, in which interactions and interdependence across scales from the local level up to the national and international levels are accommodated (Berkes, 2010). Given the complexity, uncertainty and non-linear behaviour of TEK threats, successful governance requires a full range of knowledge and skills that is difficult for any single group or 
agency to possess (Berkes, 2010; Young, 2008). Because TEK threats come from multiple scales, an approach of cross-scale institutional cooperation, in which governments, civil society and private actors are all involved in decisions, would be ideal for achieving effective conservation efforts (Bodin and Crona, 2009; Young et al., 2008).

The Inner Mongolia case study specifically highlights the necessity of cross-scale institutional arrangements that are linked both vertically (across organisations) and horizontally (across regions) (Ostrom et al., 2009). Vertically, the cooperative movement was initiated by community level organisations due to the stress generated by land degradation, poverty and other social conflicts. The cooperative movement that occurred at the community level was observed by regional/local governments; then the feedback was transmitted to the central government through the government system. The central government established the Cooperative Law and relevant policies as a response to the cooperative movement. The regional/local governments also played their roles in implementing the law and measures, which benefited cooperative activities at the community level. In this vertical interplay, though every actor has its important role to play, the central government and community play the most vital roles in determining the success of the TEK conservation efforts, as well as resource management practices.

Alongside the vertical institutional linkages, horizontal institutional linkages were applied to build a network of collaboration among communities, which involved several cooperative organisations and NGOs in Inner Mongolia. The sharing of information and experience of organisational management, funding applications, future planning, and sheep breeding provided community cooperatives a great opportunity to develop their capacity. Through this network, a horizontal, cross-organisational collaboration was established.

Another key finding from this case study is that the cross-scale institutional linkages can be positive or negative in terms of impact. While the Cooperative Law and its supporting measures have functioned as a positive support to Mongolian traditional herding practices and traditional institutions, multiple historical government policies (e.g. settlement, privatisation, Han immigration and industrial development) placed 
significant negative impacts on TEK at the community level. This finding is echoed in by many previous studies (e.g. Agrawal and Gibson, 1999; Anderies et al., 2004; Berkes, 2003, 2007), which emphasise that positioned in a complex, interdependent SES, the community level institutions are severally affected by the actions from other levels of the system, especially national level policy and legislation, both positive and negative. The study then suggests that, in an ideal scenario, the interactions and interdependence between different levels of institutions are well understood by all stakeholders of a resource system; and the community level organisations are empowered by the governmental authorities. Meanwhile, the importance of international agreements which place pressure on national government policy regarding TEK value and TEK conservation should also be noted. Alongside the international linkages, resources of funding and capacity building have been distributed to grounded levels in many cases (Berkes, 2002; Young, 2006).

\subsection{Power relations within the TEK system}

This thesis, in line with the ideology of resource governance decentralisation (e.g. Agrawal and Ostrom, 2001; Berkes, 2010; Brondzio et al., 2009; Lemos and Agrawal, 2006; Ostrom and Janssen, 2004), promotes the necessity of power devolution from the centralised state authority to lower levels of the institutional system, and appropriate power sharing among institutional actors. Cooperation among different levels of organisations and among different groups at the same scale requires appropriate power sharing (Colfer and Capistrano, 2005). The analysis of the interconnectivity and causal relationships among TEK threats illustrates that TEK and its holders are affected by various political, social, economic and cultural forces from the outside world. Unfortunately, too often, TEK holders are positioned as powerless and passive in the system (Banks, et al., 2003; Gragson and Blount, 1999; Hanna et al., 1996; Posey and Dutfield, 1996).

The Bayin case provides a good example of how vulnerable traditional practices and institutions are to external disturbance, especially to governmental policies. Between the 1960s and the early 2000s, the herders did not have any chance to express themselves in the decision-making processes regarding their livelihood and well-being. 
This unequal power relationship between government authorities and the community has also been widely observed and reported by many other scholars in other case studies across the globe (e.g. Agrawal and Ostrom, 2001; Lemos and Agrawal, 2006; Ostrom and Janssen, 2004).

The TEK conservation typology offers a set of useful approaches for achieving power devolution to overcome the current imbalance in power relations. However, in most cases, it is unlikely for local communities to achieve an equitable share of power and benefits without support from external agencies. In this scenario, there is often a key role for bridging organisations, such as NGOs, to play (Bodin and Crona, 2009; Hahn et al. 2006; Kilduff and Tsai, 2003). On one hand, the TEK holders, usually the indigenous communities, may need to strengthen their capacity through multiple approaches (e.g. self-government, self-organisation, community alliance and partnership network), which will allow the communities to work in a more equitable partnership with government authorities and other external organisations (Mannion, 1996; Labonte, 1999; Popham, 1996; Simpson et al, 2003). On the other hand, the current power holders, usually the governmental authorities, will need to recognise the importance of power decentralisation, and to be consistent with the international standards that recognise and protect indigenous land and resource rights, the rights of owning and using their indigenous knowledge, and other human rights of indigenous people (AHRC, 2008; Collings, 2009; Mauro and Hardison, 2000). The potential actions include policy and legislative support to TEK and other indigenous rights, involving indigenous peoples in environmental conservation programmes, including TEK in the formal educational system and supporting indigenous customary education and so on. These actions may create a substantial external supporting system towards indigenous selfdetermination and TEK conservation. TEK conservation design should contain both actions that target to conserve aspects of TEK directly (e.g. traditional lifeways programmes), and enabling actions that target to create wider support for TEK holders and indigenous rights (e.g. indigenous capacity building).

In most cases (Inner Mongolia is no exception), recent history of colonisation has led to the local communities losing power over government, land tenure and resource rights. Nonetheless, this trajectory does not have to be permanent. The recent changes in 
Chinese policy provide an example how small changes in government policy can be used by indigenous groups to revitalise TEK and to gain more power over resource management, regardless of whether or not this was the government's intention in designing legislation.

\subsection{Practical values of the TEK typologies}

TEK typologies provide a common language for TEK conservation actors to identify problems and resolutions, and enable them to communicate more effectively across projects (Salafsky et al., 2008). Following the example presented in Chapter Six, TEK conservation actors can apply the typologies developed in this thesis to their sites to identify and describe what has caused TEK degradation in their sites, and to figure out what conservation responses can be undertaken.

On a larger scale, the typologies provide a common language for TEK holders, practitioners and scientists to communicate, and exchange their experiences and best practices. Although the typologies are in its first phase, it seems likely that it will encourage the information transmission and experience sharing across regions and across organisations. In this way, the typologies may effectively save conservation actors' time and efforts from repeating experiments (Zent, 2001). A unified system also offers possibility and likelihood of building common databases (Agrawal, 2002). By searching through common databases of TEK conservation projects, TEK holders and practitioners may find other projects that face common threats, and find actions that can be used in the situation. To date, there are various TEK databases existing in different countries, but most of them are at a regional or national basis. There is no unified, international database that draws an overall picture of TEK and TEK conservation globally.

This research also applied the typologies for constructing the first global pattern of TEK threats and conservation actions. This pattern will be useful for guiding national and international decision making and international funds allocation (Maffi, 2005). I consider this thesis as an initial work that will hopefully inspire further, more comprehensive analyses of the status of TEK and its conservation efforts at the global scale. 


\section{LIMITATIONS AND SUGGESTIONS FOR FUTURE RESEARCH}

During the research design, one major guiding principle was to triangulate the research results across multiple sources (i.e. literature review, questionnaire, in-depth lit review, and field case study) in an attempt to limit bias. However, this $\mathrm{PhD}$ research is limited by funds and time, and this led to some sampling issues that may produce biased results. For the global survey, there were two limitations: 1 ) the uneven number of the responses from different countries and regions makes comparing results across regions more difficult; and 2) the survey and interviews were mostly conducted with researchers, and thus lack first-hand information directly from TEK holders, which may lead to potential bias.

In terms of further research, the primary need is for more studies that rigorously discuss TEK threats and conservation options, both case studies and review studies. This is especially true for studying conservation actions. To date, our understanding of TEK conservation options is incomplete, and the applicability and effectiveness of different conservation actions within different contexts is largely unknown. This causes difficulty for practitioners and decision-makers when making action plans for TEK conservation.

In an ideal scenario, I would suggest building a global network (more likely to be organised and funded by international agencies, such as the UNPFII and the CBD) of TEK monitoring and conservation, in which each country could apply standard methods and procedures of data collection and data analysis. The results from different countries and regions could then be synthesised and feedback gathered, and best practices could be adapted to the changing conditions present across the network.

This research made initial linkages between threats and specific actions and provided examples of how certain actions may address particular threats from a theoretical perspective. However, there is a further need to study the degree to which different actions can reduce particular threats over time, and to ensure efficiency of conservation action. To date, this approach has not been taken in the field of TEK research. Studies from biodiversity conservation provide useful models in this respect (e.g. Balmford et al., 
2009; Slafsky et al, 2008, 2009). There are strong links and similarities between the conservation of biological and cultural diversity. The development of the TEK typologies has been inspired by the IUCN-CMP's Classifications of Threats and Actions to Biodiversity created by Salafsky and his colleagues over the last decade (Salafsky et al., $2002,2003,2008,2009)$. The IUCN classification provides a salient example of how to link threats and actions into a unified classification system, and how this is useful and essential to the success of conservation efforts through a global network (Salafsky et al, 2008, 2009).

For the Inner Mongolian case study, it is worth noting that the results are context specific. Despite the likely applicability of these results to other parts of Inner Mongolia, and perhaps other regions of China, wider application would need to be considered in a careful manner. The cooperative activities have only been active for less than a decade, but understanding their long term effects on recovering traditional institutions and wider aspects of TEK, as well as on community empowerment, require long-term monitoring and further research. So far, this research has been the first one to focus on government policy and legislation's effects on TEK and TEK conservation in Inner Mongolia. I hope it may inspire further research that may substantially fill the research gap of TEK and TEK conservation in China. It is also worth noting that, the major goal of the Cooperative Law is not TEK revitalisation. Thus in some way, this case study presents an example of how communities subverted the laws initial intention to achieve something (e.g. institutional change and revitalisation) that has not been possible up to this point due to government repression.

\section{FINAL REFLECTION}

Addressing TEK change and degradation will require a complex, adaptive and systembased approach. Through the presentation and analysis of global patterns in TEK threats and conservation actions, this research clearly indicates the importance and urgency of power decentralisation and cooperative institutional arrangements across the space and level of organisations. There is no single indigenous community or single organisation that can save TEK on its own. Rather, cooperation and collective efforts from all levels and components of the inter-connected, social-ecological systems is the key to achieve 
effective TEK conservation and indigenous rights. In addition, in order to conserve TEK and empower the indigenous peoples, indigenous societies will need to build or re-build their capacity, as well as recognition and support from upper levels of the institutions and outside, non-indigenous societies.

To sum up, this research demonstrates the importance of TEK for indigenous societies and broader humanity. It also highlights the critical need for rapid and adaptive actions that seek to empower TEK holders and enable conditions that will ensure TEK's viability into the future. As I have addressed several times throughout this thesis, this research should be considered as an initial assessment of TEK threats and conservation on a global scale. I hope this thesis will inspire further and more comprehensive examinations of TEK threats and conservation actions across the globe. 


\section{REFERENCES}

\section{A}

Ade, L.H. (2005). From Nomadics to Settlement [in Chinese]. Wulu'muqi: Xinjiang People's Press.

Adhikari, B. (2005). Poverty, Property Rights and Collective Action: Understanding the Distributive Aspects of Common Property Resource Management. Environment and Development Economics 10: 7-31.

Adhikari, B. and Lovett, J.C. (2006a). Institutions and Collective Action: Does Heterogeneity Hinders Community-Based Resource Management? Journal of Development Studies 42 (3): 426-445.

Adhikari, B. and Lovett, J.C. (2006b). Transaction Costs and Community-Based Natural Resource Management in Nepal. Journal of Environmental Management 78 (1):515.

Adler, E. and Bernstein, S. (2005). Knowledge in Power: The Epistemic Construction of Global Governance. In M. Barnet and R. Duvall (Eds.) Power in Global Governance (pp. 294-319). Cambridge: Cambridge University Press.

Agrawal, A. (1995a). Indigenous and scientific knowledge: some critical comments. Indigenous Knowledge and Development Monitor 3(3):3-6.

Agrawal, A. (1995b). Dismantling the divide between indigenous and scientific knowledge. Development and Change 26: 413-439.

Agrawal, A. (2002a). Indigenous knowledge and the politics of classification. International Social Science Journal 54 (173): 287-297.

Agrawal, A. (2002b). Common Resources and Institutional Sustainability. In E. Ostrom, T. Dietz, N. Dolsak, P.C. Stern, S. Stonich and E.U. Weber (Eds.), Committee on the Human Dimensions of Global Change, National Research Council, Drama of the Commons (pp.41-86). Washington, DC: National Academies Press.

Agrawal, A. (2003). Sustainable governance of common-pool resources: Context, methods, and politics. Annual Review of Environment and Resources 32: 243-262.

Agrawal, A., and Gibson, C. C. (1999). Enhancement and disenchantment: The role of community in natural resource conservation. World Development 27(4): 629-649.

Agrawal, A. and Ostrom E. (2001). Collective action, property rights and decentralisation in resource use in India and Nepal. Politics and Society 29(4):485-514.

AHRC (Australian Human Rights Commission) (2008). Chapter 7 - The protection of Indigenous Knowledge in The Title Report. Accessed on $5^{\text {th }}$ March 2012 via 
http://www.humanrights.gov.au/social justice/nt report/ntreport08/pdf/ch ap7.pdf

Ahren, M. (2002). An introduction to the WIPO Intergovernmental Committee on Intellectual Property, Genetic Resources, Traditional Knowledge and Expressions of Folklore. Indigenous Affairs, International Processes: Perspectives and Challenges, 1(02):64-70. Copenhagen: IWGIA. Available online at http://www.iwgia.org

Ajzen, I. and Fishbein, M. (1980). Understanding Attitudes and Predicting Social Behaviour. Upper Saddle River, NJ: Prentice Hall.

Akinnifesi, F.K., Chirwa, P.W., Ajayi, O.C., Sileshi, G., Matakala, P., Kwesiga, F.R., Harawa, H. and Makumba, W. (2008). Contributions of agroforestry research to livelihood of smallholder farmers in Southern Africa: Taking stock of the adaptation, adoption and impact of fertiliser tree options. Agricultural Journal 3 (1): 58-75

Albuquerque, U.P. (2006). Re-examining hypotheses concerning the use and knowledge of medicinal plants: a study in the Caatinga vegetation of NE Brazil. Journal of Ethnobiology and Ethnomedicine 2:30.

Alcorn, J. B. (1989). Process as resource. Advances in Economic Botany 7:63-77.

Alcorn, J. B. (1993). Indigenous peoples and conservation. Conservation Biology 7:424426.

Alcorn, J.B. (2000). Indigenous peoples and conservation organisations: experiences in collaboration. Washington, D.C: World Wildlife Fund (USA).

Alcorn, J.B. and Toledo, V. M. (2000). Resilient resource management in Mexico's forest ecosystems: The contribution of property rights. In F. Berkes, C. Folke and J. Colding (Eds.), Linking Social and Ecological Systems: Management Practices and Social Mechanisms for Building Resilience (pp. 216-249). Cambridge: Cambridge University Press.

Altieri, M.A. (1994). Biodiversity and Pest Management in Agroecosystems. New York: Haworth Press.

Anaya, J. (1996). Indigenous Peoples in International Law. New York, USA: Oxford University Press.

Anderies, J., Janssen, M. and Ostrom, E. (2004). A framework to analyse the robustness of social-ecological systems from an institutional perspective. Ecology and Society 9(1): Article 18.

Anderies, J.M., Ryan, P. and Walker, B.H. (2006). Loss of resilience, crisis, and institutional change: Lessons from an intensive agricultural system in southeastern Australia. Ecosystems 9(6):865-878. 
Ao, R.Q. (2003). The Changing and Bring Forth New Ideas of the Property in Grassland [in Chinese]. Inner Mongolia Social Science 4: 24-35.

Ao, R.Q. (2005). Institutional Change and Pastoral Civilisation [in Chinese]. Huhehaote, China: Inner Mongolia People's Press.

Ao, R.Q. (2008). Review of Institutional and Policy Change in Grassland Collective Economic Development [in Chinese]. Paper presented at the Grassland Cooperative Development Forum, Huhehaote, Inner Mongolia, China.

Ao, R.Q. (2009).The changes of the system of grassland using and ownership [in Chinese]. The collection of the proceeding papers of the Forum of China and Mongolia Cooperative Projects 2009: Ulan Bator.

APFB (Alashan Prefecture Forestry Bureau). (2002). The Annual Report of the Alashan Prefecture Forestry Bureau (2001) [in Chinese]. Bayanhaote: Alashan Prefecture Forestry Bureau.

APFB (Alashan Prefecture Forestry Bureau). (2006). The Annual Report of the Alashan Forestry Bureau (2005) [in Chinese]. Bayanhaote: Alashan Prefecture Forestry Bureau.

Armitage, D., Marschke, M. and Plummer, R. (2008). Adaptive co-management and the paradox of learning. Global Environmental Change 18: 86-98.

Ascher, W. (1999). Why Governments Waster Natural Resources: Policy Failures in Developing Countries. Baltimore: The Johns Hopkins University Press.

Assimeng, J.M. (1997). Traditional leader's capacity and disposition for democracy: The example of Ghana. In W. Hofmester and I. Scholz (Eds), Traditional and Contemporary Forms of Local Participation and Self-governance in Africa. Johasonburg: KAF.

B

Ba, Y. (2008). The development of herders' cooperative organisations in Grassland [in Chinese]. Bayin Haote, Inner Mongolia, China: The Ha'ri'gen'tai Animal Byproducts Association.

Ba, Y. (2010). The project of community development in grassland, Inner Mongolia [in Chinese]. Bayin Haote, Inner Mongolia, China: The Ha'ri'gen'tai Animal Byproducts Association.

Baker, E. and Teaser-Polk, C. (1998). Measuring community capacity: Where do we go from here? Health Education and Behaviour 25 (3): 279-283.

Balick, M. J. and Cox, P. A. (1996). Plants, People, and Culture: The Science of Ethnobotany. New York: Scientific American Library. 
Banks, J.S. and Duggan, J. (2000). A bargaining model of collective choice. American Political Science Review 94: 73-88.

Banks, T., Richard, Camille, R., Li, P. and Yan, Z.L. (2003). Community-Based Grassland Management in Western China Rationale, Pilot Project Experience, and Policy Implications. Mountain Research and Development 23(2):132-140.

Bao, X.X., Yi, J., and Jiri, J.S. (2009). The effects of different herding practices to stipa grandis steppe communities on the Mongolian steppe [in Chinese and Mongolian]. The collection of the proceeding papers of the Forum of China and Mongolia Cooperative Projects 2009: Ulan Bator.

Barnett, M. and Duvall, R. (2005). Power in global governance. Cambridge: Cambridge University Press.

Barraclough, S. and Finger-Stich, A. (1996). Some ecological and social implications of commercial shrimp farming in Asia. United Nations Research Institute for Social Development. UNRISD Discussion paper No.74.

Battiste, M.A. and Henderson, J.Y. (2000). Protecting Indigenous Knowledge and Heritage: A global Challenge. Saskatton, SK: Purish Press Publishing Ltd.

Becker, C. D. and Ghimire, K. (2003). Synergy between traditional ecological knowledge and conservation science supports forest preservation in Ecuador. Conservation Ecology 8(1): Article 1. [online] URL: http://www.consecol.org/vol8/iss1/art1/

Begossi, A., Hanazaki, N. and Tamashiro, J. (2002). Medicinal plants in the Atlantic Forest (Brazil): Knowledge, use, and conservation. Human Ecology 30(3):281-299.

Beisner, B.E., Haydon, D.T. and Cuddington, K. (2003). Alternative stable states in ecology. Front Ecology Environment 1: 376-382.

Bene, C. and Neiland, A.E. (2006) From Participation to Governance. Penang, Malaysia: World Fish Center and Colombo, Sri Lanka: CGIAR Challenge Program on Water and Food.

Benz, B., Cevalloz, E. J., Santana, M. F., Rosales, A. J. and Graf, S. (2000). Losing knowledge about plant use in the sierra de manantlan biosphere reserve, Mexico. Economic Botany 54(2):183-191.

Berkes, F. (Ed.) (1989). Common Property Resources: Ecology and Community-based Sustainable Development. London: Belhaven.

Berkes, F. (2000). Learning to design resilient resource management: indigenous systems in the Canadian subarctic. In F. Berkes and C. Folke (Eds.), Linking Social and Ecological System: Management Practices and Social mechanisms for Building Resilience (pp. 98-128). Cambridge, UK: Cambridge University Press. 
Berkes, F. (2002). Cross-scale institutional linkages: Perspectives from the bottom up. In E. Ostrom, T. Dietz, N. Dolsak, P. C. Stern, S. Stonich and E. U. Weber (Eds.), The Drama of the Commons (pp. 293-321). Washington: National Academy Press.

Berkes, F. (2007). Community-based conservation in a globalised world. National Academy of Sciences of the USA 104(39):15188-15193.

Berkes, F. (2008). Sacred ecology. Traditional ecological knowledge and resource management. Philadelphia and London, UK: Taylor and Francis,

Berkes, F. (2009). Evolution of co-management: Role of knowledge generation, bridging organisations and social learning. Journal of Environmental Management 90:1692-1702.

Berkes, F. (2010). Devolution of environment and resource governance: Trends and future. Environmental Conservation 37(4):489-500.

Berkes, F., and Folke, C. (1998). Linking Social and Ecological Systems: Management Practices and Social Mechanisms for Building Resilience. Cambridge: Cambridge University Press.

Berkes, F., Bankes, N., Marschke, M., Armitage, D., and Clark, D. (2005). Cross-scale institutions and building resilience in the Canadien North. Calgary: University of Calgary Press.

Berkes, F., C. Folke, and M. Gadgil. (1995). Traditional ecological knowledge, biodiversity, resilience and sustainability. In C. Perrings, K.-G. Ma"ler, C. Folke, C. S. Holling, and B.-O. Jansson (Eds.) Biodiversity conservation. Dordrecht, the Netherlands: Kluwer Academic Publishers.

Berkes, F., M. Kislalioglu, C. Folke, and M. Gadgil. (1998). Exploring the basic ecological unit: ecosystem-like concepts in traditional societies. Ecosystems 1:409-415.

Berkes, F., Colding, J. and Folke, C. (2000). Rediscovery of traditional ecological knowledge as adaptive management. Ecological Application 10(5):1252-1262.

Berkes, F., Colding, J and Folke, C. (Eds.) (2003). Navigating Social-Ecological Systems: Building Resilience for Complexity and Change. Cambridge: Cambridge University Press.

Berlin, B. (1976). The concept of rank in ethnobiological classification: some evidence from Aguaruna folk botany. American Ethnologist 3(3):381-99.

Berlin, B. (1992). Ethnobiological Classification: Principles of Categorization of Plants and Animals in Traditional Societies. New Jersey: Princeton University Press.

Berlin, B., Boster, J. and O’Neill, J.P. (1981) The Perceptual Bases of Ethnobiological Classification: Evidence from Aguaruna Jivaro Ornithology. Journal of Ethnobiology 1(1):95-108. 
Bernard, H. R. (2011). Research Methods in Anthropology: Qualitative and Quantitative Approaches (5th edition). Plymouth, UK: AltaMira Press.

Bertram, I.G. (1987). The Political economy of decolonisation and nationhood in small pacific societies. In S. Britton Hooper, R. Crocombe, J. Huntsman, C. Macpherson (Eds.), Class and Culture in the South Pacific. Suva, Fiji: Centre for South Pacific Studies and Institute of Pacific Studies.

Bidwell, R. D. and Ryan, C.M. (2006). Collaborative Partnership Design: The Implications of Organizational AffIliation for Watershed Partnerships. Society and Natural Resources 19(9): 827 - 843.

Binder, L. and Hanbidge, B. (1993). Aboriginal people and resource co-management: the Inuvialuit of the Western Arctic and resource co-management under a land claims settlement. In J.T. Inglis (Ed.), Traditional Ecological Knowledge: Concepts and Cases (pp121-132). Ottawa: International Program on Traditional Ecological Knowledge \& International Development Research Centre.

Bishop, R. and Glynn, T. (2003). Culture Counts: Changing Power Relations in Education. Palmerston North, New Zealand: Zed Books.

Blaikie, P. and Jeanrenaud, S. (1997). Biodiversity and human welfare. In K. B. Ghimire, K. Ghimire and M. P. Pimbert. Social Change and Conservation: Environmental Politics and Impacts of National Parks and Protected Areas (pp. 46-70). London: Earthscan.

Blann, K., Light, S., and Musumeci, J. A. (2003). Facing the adaptive challenge: practitioners' insights from negotiating resource crises in Minnesota. In F.Berkes, J.Colding and C. Folke (Eds.), Navigating the dynamics of social-ecological systems (pp. 210-240). Cambridge: Cambridge University Press.

Bodin, Ö. and Crona, B.I. (2009). The role of social networks in natural resource governance: What relational patterns make a difference? Global Environmental Change 19: 366-374.

Bogdan, R., and Biklen, S. K. (2006). Qualitative Research for Education: An Introduction to Theories and Methods (5th Edition). Boston: Allyn \& Bacon.

Bocking, S. (2004). Nature's experts: science, politics, and the environment. New Brunswick, NJ: Rutgers University Press.

Boom, B. (1987). Ethnobotany of the Chacobo Indians, Beni, Bolivia. Advances in Economic Botany 4:1-68.

Bonjour, L. (1985). The Structure of Empirical Knowledge. Cambridge University Press. 
Borrini-Feyerabend, M., Pimbert, M., Farvar, M .T., Kothari, A. and Renard, Y. (2004) Sharing Power: Learning by Doing in Co-management on Natural Resources throughout the World. IIED and IUCN/CEESP. Teheran: Cenesta Publishing.

Boster, J.S. (1986). Agreement between biological classification systems is not dependent on cultural transmission. American Anthropologist 89(4):914-920.

Boster, J.S. and D'Andrade, R. (1989). Natural and Human Sources of Cross-Cultural Agreement in Ornithological Classification. American Anthropologist 91(1):132142.

Boster, J. S. and Johnson, J.C. (1989). Form or function: A comparison of expert and novice judgements of similarity among fish. American Anthropologist 91:866-889.

Bressen, T. (2007). Consensus decision-making. In P. Holman, T. Devane and S. Cady (Eds.) The Change Handbook. San Francisco: Berrett-Koehler Publishers Inc.

Brodt, S. (2001). A systems perspective on the conservation and erosion of indigenous agricultural knowledge in Central India. Human Ecology 29(1):99-120.

Brondizio, E. S., Ostrom, E. and Young, O. R. (2009). Connectivity and the governance of multilevel social-ecological systems: The role of social capital. Annual Review of Environment and Resources 34: 253-278.

Brown, K. (2002). Innovation for conservation and development. The Geographical Journal 168(1): 6-17.

Bruce, D. (2003). Insights on Community Capacity Building. Presentation available at dwbruce@mta.ca

Brugere, C. (2006). Can integrated coastal management solve agriculture-fisheriesaquaculture conflicts at the land-water interface? In C.T. Hoanh, T.P. Tuong, J.W. Gowing and B. Hardy (Eds.), Environment and Livelihoods in Tropical Coastal Zones (pp. 258-273). Oxon, UK: CAB International.

Brunner, R.D., Steelman, T.A., Coe-Juell, L., Cromley, C.M., Edwards, C.M. and Tucker, D.W., (Eds.). (2005). Adaptive Governance: Integrating science, policy and decision making. New York: Columbia University Press.

Bruno, D. and Richmond, H. (2003). The truth about taxonomies. Information Management Journal 37(2):44.

Brush, S.B. (2005). Famers' rights and protection of traditional agricultural knowledge. CGIAR System wide Program on Collective Action and Property Rights, working paper No. 36

Bryant, R.L. (1998). Power, knowledge and political ecology in the third world: a review. Progress in Physical Geography 22(1): 79-94. 
Bryman, A. (2008). Social research methods (3rd Edition). Oxford: Oxford University Press.

Butler, R. and Hinch, T. (Eds.) (2007). Tourism and Indigenous Peoples: Issues and Implications. Oxford: Elsevier Ltd.

Butler, C. F. and Menzies, C.R. (2007). Traditional ecological knowledge and indigenous tourism. In R. Butler and T. Hinch (Eds.), Tourism and Indigenous Peoples: Issues and Implications (pp. 15-27). Oxford: Elsevier Ltd.

Byg, A. and Balslev, H. (2001). Traditional knowledge of Dypsis fibrosa (Aracaceae) in Eastern Madagascar. Economic Botany 55:263-275.

C

Caniago, I. and Siebert, S.F. (1998). Medicinal plant economy, knowledge and conservation in Kalimantan, Indonesia. Economic Botany 52:229-250.

Cao, J.J., Xiong, Y.C., Sun, J., Xiong, W.F. \& Du, G.Z. (2011). Differential benefits of multi-and single-household grassland management patterns in the Qinghai-Tibetan plateau of China. Human Ecology 39: 217-27.

Capra, F. (1996). The web of life. New York: Anchor Books.

Carlsson, L. (2000). Policy networks as collective action. Policy Studies Journal 28: 502520.

Carlsson, L. and Berkes, F. (2005) Co-management: concepts and methodological implications. Journal of Environmental Management 75: 65-76.

Carson, D. (1999). Community-based education for indigenous cultures. In S. May, (Ed.), Indigenous Community-based Education (pp 8-19). PA: Multilingual Matters Ltd.

Case, R.J., Pauli, G.F. and Soejarto, D.D. (2005). Factors in maintaining indigenous knowledge among ethnic communities of Manus Island. Economic Botany 59:356-365.

Casswell, S. (2001) Community capacity building and social policy - What can be achieved? Social Policy Journal of New Zealand 17: 22-35.

Cavalli-Sforza, L. L., Feldman, M. W., Chen, K. H. and Dornbusch, S. M. (1982). Theory and Observation in cultural transmission. Science 218(4567): 19-27.

Cavaye, D.J. (1999). The role of government in community capacity building. Draft report prepared for the Department of Primary Industries, Queensland, Australia. 
CBD (Convention on Biological Diversity) (2006). Article 8: In-Situ Conservation. Accessed on $6^{\text {th }}$ March 2012 via http://www.cbd.int/convention/articles/?a=cbd-08

CBD (Convention of Biological Diversity). (2011). Country profile - China. Accessed on 17th September 2011 via http://www.cbd.int/countries/?country=cn

Central Government of China. (1982). Constitution of China [in Chinese]. Chapter 1, Article 4. Accessed on $5^{\text {th }}$ October 2011 via http://www.gov.cn/gongbao/content/2004/content 62714.htm

Central government of China. (1984). The Chinese Ethnic Minority Law. [in Chinese]. Accessed on $5^{\text {th }}$ October, 2011 via http://www.gov.cn/test/2005$\underline{07 / 29 / \text { content 18338.htm. }}$

Chambers, R. (1983). Rural Development: Putting the Last First. London: Longman.

Charmaz, K. (2006). Constructing Grounded Theory: A Practical Guide Through Qualitative Analysis. CA: Sage Publications.

CMP (Conservation Measures Partnership). (2004). The open standards for the practice of conservation actions. Washington, D.C: CMP. Accessed on $4^{\text {th }}$ October 2011 via http://www.conservationmeasures.org/wpcontent/uploads/2010/04/CMP Open Standards Version 2.0.pdf

Cinner, J.E. and Aswani, S. (2007). Integrating customary management into marine conservation. Biological Conservation 140: 201-216.

CMP (Conservation Measures Partnership). (2005). Taxonomies of direct threats and conservation actions. Washington, D.C.: CMP.

CMP (Conservation Measure Partnership). (2010). Cycle of adaptive management. Open domain source. Accessed on $4^{\text {th }}$ February 2012 via http://commons.wikimedia.org/wiki/File\%3ACMP_Cycle_-_2008-02-20.jpg

CMP-IUCN (Conservation Measures Partnership-International Union for the Conservation of Nature) (2007). Measuring threat magnitude: a comparison of existing systems and a proposal for a standard system. Working paper. Washington, D.C.: CMP \& Gland, Switzerland: IUCN.

Cofer, D.A. (2000). Informal workplace learning (Practical Application Brief No.10). Columbus, OH: Center on Education and Training for Employment.

Colchester, M. (1994). Salvaging nature: Indigenous people, protected areas and biodiversity conservation. Geneva: UNRISD Discussion paper No.55.

Colchester, M.(2000). Self-determination or environmental determination for indigenous peoples in tropical forest conservation. Conservation Biology 14(5):1365-1367. 
Colchester, M., and Erni, C. (Eds). (1999). Indigenous peoples and protected areas in South and Southeast Asia: from principles to practice. Document No. 97. Forest Peoples Programme and the International Work Group for Indigenous Affairs, Copenhagen.

Colding, J. (1998). Analysis of hunting options by the use of general food taboos. Ecological Modelling 110:5-17.

Colfer, C. J. P. and Capistrano, D. (2005) The Politics of Decentralization: Forests, People and Power. London, UK: Earthscan.

Collings, N. (2009). Chapter III. Environment. In DESA, State of the World's Indigenous Peoples (pp. 84-128). UN Doc. Available at http://www.un.org/esa/socdev/unpfii/documents/SOWIP web.pdf

Cordell, J. (1995). Review of traditional ecological knowledge. Journal of Political Ecology 2: 43-47.

Costa-Pierce, B.A. (1987). Aquaculture in Ancient Hawaii. Bioscience 37: 320-330.

Cowling, R. M. and Pressey, R. L. (2003). Introduction to systematic conservation planning in the Cape Floristic Region. Biological Conservation 112:1-13.

Cox, P. (2000). Will tribal knowledge survive the millennium? Science 287(5450): 44-45.

Craig, G. (2007). Community capacity-building: Something old, something new ...? Critical Social Policy 27(3): 335-359.

Croquant (2007). The location of Huhhot Prefecture within Inner Mongolia Autonomous Region of China. Wikipedia Open Source. Retrieved on 6th September, 2010 via http://en.wikipedia.org/wiki/File:Location of Hohhot Prefecture within Inner Mongolia (China).png

Cruickshank, J. (2002). Realism and sociology: Anti-foundationalism, ontology and social research. Oxford: Routledge

Cruz García, G.S. (2006). The mother - child nexus. Knowledge and valuation of wild food plants in Wayanad, Western Ghats, India. Journal of Ethnobiology and Ethnomedicine 2:39-45.

Crystal, D. (2000). Language Death. Cambridge: Cambridge University Press.

D

Da, L. T. (2003a). A review of grassland use in Inner Mongolia in the last 50 years [in Chinese]. Paper presented at the Tian Xia Rostrum Conference, Beijing, 26 March, 2006. 
Da, L. T. (2003b). A study of the non-equilibrium ecosystem in Inner Mongolia grassland [in Chinese]. The Friends of Nature 3: 34-39.

Da, L. T. (2004). Rethinking grassland desertification: Theory and the systems [in Chinese]. The Friends of Nature 2: 12-17.

Da, L. T. (2008). The effects of institutional and policy changes to grassland ecosystems in Inner Mongolia [in Chinese]. In Y.S. Zheng (Ed), A Review of the Environment and Development in China. Beijing: Social Science Academy Press.

Da, L. T. and Si, H. (2006). The causes of desertification in Inner Mongolia [in Chinese]. Paper presented to the Friends of Nature Forum. Beijing.

Da, L.T. and Zheng, Y. S. (2010). Pastoral Areas and the Market: Pastoral Economy [in Chinese]. Beijing: The Centre for Rural Environment Social Studies.

Daes, E.I.A. (1999). Human rights of indigenous peoples: Indigenous people and their relationship to land. Second progress report on the working paper. Subcommission on Prevention of Descrimination and Protection of Minoroties, United Nations Commission on Human Rights. Geneva, Switzerland.

Daoas, D.A. (1995). The rights of the cultural communities in the Philippines. Conference proceedings. International Work Groups for indigenous Affairs (IWGIA) document. 80, 97-107, 102-103.

Dei, G. J. S. (1993). Indigenous African knowledge systems: local traditions of sustainable forestry. Singapore Journal of Tropical Geography 14:28-41.

Dei, G.J.S., Hall, B. L. and Rosenberg, D.G. (2000). Indigenous Knowledge in Global Contexts. Toronto: University of Toronto Press.

DESA (Department of Economic and Social Affairs of UN). ( 2009). The Status of World's Indigenous Peoples. UN Doc. Accessed $5^{\text {th }}$ March 2012 via http://www.un.org/esa/socdev/unpfii/documents/SOWIP web.pdf http://www.un.org/esa/socdev/unpfii/documents/6 session dodson.pdf

DEWHA (Department of the Environment, Water, Heritage and the Arts of Australian Government) (2009). Indigenous knowledge management systems: Guide for indigenous communities. Govt Doc. Accessed on $5^{\text {th }}$ August 2012 via http://www.environment.gov.au/indigenous/publications/knowledgemanagement-guide.html

Dickason, O.P. (1989). Concepts of souvereignty at the time of first contacts. In L.C. Green and O.P. Dickason, The Law of Nations and the New World (pp. 141-249). Edmonton, Canada: University of Alberta Press.

Dillman, D. A. (2000). Mail and Internet Surveys: The Tailored Design Method. New York: John Wiley \& Sons, Inc. 
Dobson, A. and Eckersley, R. (2006). Political theories and ecological challenges. Cambridge: Cambridge University Press.

Dorough, D.S. (2009). Chapter VI: Human rights. In DESA of UN, State of World's Indigenous Peoples (pp52-81). United Nations Document. New York, USA.

Dowie, M. (2006). Conservation Refugees. In J. Mander and V. Tauli-Corpuz. (Eds.), Paradigm Wars: Indigenous Peoples' Resistance to Globalization. A special Report of the International Forum on Globalization Committee on Indigenous Peoples. San Francisco, CA.: Sierra Club Book.

Drengson, A.R. and Inoue, Y. (1995). The deep ecology movement: an introductory anthology. Berkeley, CA: North Atlantic Books.

Drew, J. A. (2005). Use of traditional ecological knowledge in marine conservation. Conservation Biology 19(4): 1286-1293.

Duerden, F. and Kuhn, R.G. (1998). Scale, context, and application of traditional knowledge of the Canadian north. Polar Record 34(188):31-38.

Dutfield, G. (2000a). The Public and Private Domains Intellectual Property Rights in Traditional Knowledge. Science Communication 21(3):274-295.

Dutfield, G. (2000b). Intellectual property rights, trade and biodiversity: Seeds and plant varieties. Oxford: Earth Scan.

Dutfield, G. (forthcoming). The potato park as a sui generis system for the protection of traditional knowledge.

$\mathbf{E}$

Eer, D.M.T (2007). The Study of Mongolian Traditional Knowledge of Edible Wild Plants In Eerdos Plataea [in Chinese]. Masters thesis submitted to Inner Mongolia Normal University, China.

Ellen, R. F. (2007). Modern Crises and Traditional Strategies: Local Ecological Knowledge in Islands Southeast Asia (Vol. Studies in Environmental Anthropology and Ethnoecology). New York: Berghahn Books.

Ellen, R., Parkes, P. and Bicker, A. (Eds.). (2000). Indigenous Environmental Knowledge and its Transformations: Critical Anthropological Perspectives. Amsterdam: Harwood Academic Publishers Ltd.

Ember, C.R. and Ember, M. (2001). Cross-cultural Research Methods. MA: AltaMira Press.

En, H. (2005). Cultural review of desertification on the Mongolian steppes [in Chinese]. Inner Mongolian Social Science 3: 21-27. 
Enkhee (2009). The essence of Haote Aili institutional system and its comtemporary transmission [in Chinese and Mognolian]. The collection of the proceeding papers of the Forum of China and Mongolia Cooperative Projects 2009: Ulan Bator.

Epstein, J. H., Olival, K. J., Pulliam, J. R., Smith, C., Westrum, J., Hughes, T., Dobson, A. P., Zubaid, A., Rahman, S. A., Basir, M., Field, H. E. and Daszak, P. (2009). Pteropus vampyrus, a hunted migratory species with a multinational home-range and a need for regional management. Journal of Applied Ecology 46: 991-1002.

Eraut, M. (2004). Informal learning in the workplace. Studies in Continuing Education 26(2):247-273.

Eversole, R. (2011). Community agency and community engagement: Re-theorising participation in governance. Journal of Public Policy 31(1):51-71.

Eyssartier, C, Ladio, A.H, and Lozada, M. (2008). Cultural transmission of traditional knowledge in two populations of North-western Patagonia. Journal of Ethnobiology and Ethnomedicine. Online http://www.ncbi.nlm.nih.gov/pubmed/19077315

Ezzy, D. (2002). Qualitative Analysis: Practice and Innovation. Crows Nest, NSW: Routledge.

\section{$\mathbf{F}$}

Farah, M. I. (1993). From ethnic response to clan identity: A study of state penetration among the Somali nomadic pastoral society of northern Kenya. Stockholm: Almqvist and Wiksell.

Fernandez-Gimenez, M. E. (2000). The role of Mongolian pastoralists' ecological knowledge in rangeland management. Ecological Applications 10(5): 1318-1326.

Fernandez-Gimenez, M.E. (2002). Spatial and social scales and boundaries: Implications for managing pastoral land use in Mongolia. Human Ecology 30(1): 49-78.

Fettes, M. (1999). Indigenous education and the ecology of community. In S. May, (Ed.) Indigenous Community-based Education (pp 20-41). PA: Multilingual Matters Ltd.

Florey, M. (2001). Threats to indigenous knowledge: A case study from Eastern Indonesia. In L. Maffi (Ed.), On Biocultural Diversity: Linking Language, Knowledge, and the Environment (pp. 325-342). Washington, DC: Smithson. Inst. Press.

Folke, C., Berkes, F. and Colding, J. (2000). Ecological practices and social mechanisms for building resilience and sustainability. In F. Berkes, C. Folke and J. Colding (Eds.), Linking Social and Ecological Systems: Management Practices and Social 
Mechanisms for Building Resilience (pp. 414-436). Cambridge: Cambridge University Press.

Folke, C., Colding, J. and Berkes, F. (2003). Synthesis: Building resilience and adaptive capacity in social-ecological systems. In F. Berkes, J. Colding and C. Folke (Eds.), Navigating Social-Ecological Systems: Building Resilience for Complexity and Change (pp. 352-387). Cambridge, UK: Cambridge University Press.

Folke, C., Hahn, T., Olsson, P. and Norberg, J. (2005). Adaptive governance of socialecological systems. Annual Review of Environment and Resources 30: 441-473.

Folke, C. (2006). Resilience: The emergence of a perspective for social - ecological systems analyses. Global Environmental Change 16:253-267.

Fons J. R., Vijver, V.D. and Leung, K. (1997). Methods and Data Analysis for Cross-Cultural Research. London: SAGE.

Ford, J. and Martinez, D. (2000). Traditional ecological knowledge, ecosystem science, and environmental management. [Editorial Material]. Ecological Applications 10(5): 1249-1250.

Friederichs, K. (1958). A definition of ecology and some thoughts about basic concepts. Ecology 39(1):154-159.

Friedman, J., Yaniv, Z., Dafni, A. and Palewitch, D. (1986). A Preliminary Classification of the Healing Potential of Medicinal Plants, Based on a Rational Analysis of an Ethnopharmacological Field Survey among Bedouins in the Negev Desert, Israel. Journal of Ethnopharmacology 16:275-87.

\section{G}

Gadgil, M., Berkes, F., and Folke, C. (1993). Indigenous knowledge for biodiversity conservation. Ambio 22:151-156.

Gadgil, M., Hemam, N. S. and Reddy, B. M. (1998). People, refugia and resilience. In F. Berkes and C. Folke (Eds.) Linking social and ecological systems: management practices and social mechanisms for building resilience (pp.30-47). Cambridge, UK: Cambridge University Press.

Gadgil, M., Seshagiri Rao, P. R., Utkarsh, G., Pramod, P. and Chatre, A. (2000). New meanings for old knowledge: the people's biodiversity registers programme. Ecological Applications 10:1307-1317.

Gadgil, M., Olsson, P., Berkes, F., and Folke, C. (2003). Exploring the role of local ecological knowledge for ecosystem management: three case studies. In F. Berkes, J. Colding \& C. Folke (Eds.), Navigating social-ecological systems: building resilience for complexity and change (pp. 189-209). Cambridge: Cambridge University Press. 
Galindo-Leal, C. (2001). Design and Analysis of Conservation Projects in Latin America: an Integrative Approach to Training. Conservation Ecology 5(2): Article16. [online] URL: http://www.consecol.org/vol5/iss2/art16/

Gao, Y.J. and Deng, A. (2007). Tibetan nomadic herders' settlement and new pastoral area development [in Chinese]. Ethnic Minority Research 5: 67-78.

Gavin, M. C., Wali, A. and Vasquez, M. (2007). Parks, people, and participation: Towards collaborative and community-based natural resource management. In S. Kindon, R. Pain \& M. Kesby (Eds.), Connecting People, Participation and Place:

Participatory Action Research Approaches and Methods (pp. 60-70). London: Routledge.

Geissler, P., Harris, S.A., Prince, R., Olsen, A., Odhiambo, R.A., Oketch-Rabah, H., Madiega, P.A., Andersen, A. and Molgaard, P. (2002). Medicinal plants used by Luo mothers and children in Bondo district, Kenya. Journal of Ethnopharmacology 83:39-54.

Gerry, J. S. (1996). The Diffusion of Sovereignty: Self-Determination in the Post-Colonial Age. Stanford Journal of International Law 32: 255-271.

Ghimire, S.K., McKey, D. and Aumeeruddy, T. (2004). Heterogeneity in Ethnoecological Knowledge and Management of Medicinal Plants in the Himalayas of Nepal: Implications for Conservation. Ecology and Society 9(3): article 6.

Ginsburg, F. (1991). Indigenous media: Faustian contract or global village? Cultural Anthropology 6(1): 92-112.

Ginsburg, F. (1993). Aboriginal media and the Australian imaginary. Public Culture 1993 (5): 557-578.

Godoy, R. A., Brokaw, N., Wilkie, D. S., Colón, D., Palermo, A., Lye, S. and Wei, S. (1998). On trade and cognition: markets and the loss of folk knowledge among the Tawahka Indians of the Honduran rain forest. Anthropological Research 54: 219-233.

Godoy, R., Reyes-Garcia, V., Byron, E., Leonard, W. R. and Vadez, V. (2005). The effect of market economy on the well-being of indigenous peoples and on their use of renewable natural resources. Annual Review of Anthropology 34: 121-138.

Godoy, R., Reyes-García, V., Broeschc, J., Fitzpatrickd, I.C., Giovanninnie, P., Martínez Rodríguezf, M.R., Jhaa, N., Huancaa, T., Leonardg, W.R., and Tannerf, S. (2008). Does modernisation erode the secular trend of indigenous knowledge? New evidence from native Amazonians born 1920-1985, Bolivia. Tsimane's Amazon Panel Study Working Paper \# 29. Accessed on $5^{\text {th }}$ May 2011 via http://www.tsimane.org/working\%20papers/TAPS-WP-29.pdf

Groffman, P. M., Baron, J.S., Blett, T., Gold, A., Goodman, F., Gunderson, L.H., Levinson, B.M., Palmer, M.A., Paerl, H.W., Peterson, G.D., Poff, N.L., Rejeski, D.W., Reynolds, J.F., Turner, M.G., Weathers, K.C. and Wiens, J. (2006). Ecological thresholds: The key 
to successful environmental management or an important concept with no practical application? Ecosystems 9: 1-13.

Gohlert, E. (1993). Foundation of sustainable development. In M. Albertson, and M. Shinn (Eds.), Proceeding of the International Conference on Sustainable Village Based Development. Colorado: CSU, Fort Collins.

Gomez, N. (2007). Healing Hidden Wounds. Cultural Survival Quarterly 31(3).

Goodwin, H. (2007). Indigenous tourism and poverty reduction. In R. Butler and T. Hinch (Eds.), Tourism and Indigenous Peoples: Issues and Implications (pp. 84-94). Oxford: Elsevier Ltd.

Gragson, T. L. and Blount, B. G. (1999). Ethnoecology: Knowledge, Resources, and Rights. Athens: The University of Georgia Press.

Gray, A., Parellada, A. and Newing, H. (1998). From principles to practice: indigenous peoples and biodiversity conservation in Latin America. Document 87. Forest Peoples Programme, the Interethnic Association for the Development of the Peruvian Amazon, and the International Work Group for Indigenous Affairs, Copenhagen.

Grigorenko, E.L., Meier, E., Lipka, J., Mohatt, G., Yanez, E. and Sternberg. R.J.( 2004). The relation between academic and practical intelligence: A case study of the tacit knowledge of native American Yup'ik people in Alaska. Learning and Individual Differences 14(4):183-207.

Groffman, P.M., Baron, J.S., Blett,T., Gold, A.J., Goodman, I., Gunderson,L.H., Levinson, B.M., Palmer, M.A., Paerl, H.W., Peterson,G.D., Poff, N.L., Rejeski, D.W., Reynolds, J.F., Turner, M.G., Weathers, K.C. and Wiens, J. (2006). Ecological Thresholds: The Key to Successful Environmental Management or an Important Concept with No Practical Application? Ecosystems 9: 1-13.

Gruber, L. (2000). Ruling the World: Power Politics and the Rise of Supranational Institutions. Princeton: Princeton University Press.

Guest, G. (2002). Market Integration and the Distribution of Ecological Knowledge within an Ecuadorian Fishing Community. Journal of Ecological Anthropology 6:38-49.

Guglielmino, C.R., Viganotti, C., Hewlett, B. and Cavalli-Sforza, L.L. (1995). Cultural variation in Africa: Role of mechanisms of transmission and adaptation. Proceedings of National Academy of United States of America 92: 7585-7589.

Guha-Khasnobis, B., Kanbur, R., and Ostrom, E. (Eds.). (2006). Linking Formal and Informal Economy: Concepts and Policies. Oxford: Oxford University Press.

Gunderson, L., C.S., H., and Light, S. (Eds.). (1995). Barriers and Bridges to Renewal of Ecosystems and Institutions. New York: Columbia University Press. 
Gunderson, L. H. and Holling, C. S. (Eds.) (2002). Panarchy: Understanding

Transformations in Human and Natural Systems. Washington, DC: Island Press.

Guo, Q. S., Tan, D. Y., Wang, C. L., Shi, Z. M., and Ma, C. (2005a). Effects of Cistanche deserticola inoculation on undergrowth vegetation of natural Haloxylon ammodendron forest [in Chinese]. Ecology 8: 15-19.

Guo, Q. S., Wang, C. L., Guo, Z. H., Tan, D. Y., and Shi, Z. M. (2005b). Geographic distribution of existing Haloxylon desert vegetation and its patch character in China [in Chinese]. Forestry Science 5: 23-27.

Guo, W.H. (2010). The Issues of Nomadic Herders' Settlement in Xinjiang since the Economic Reforms [in Chinese]. Masters Thesis submitted to University of Xinjiang, China.

Guo, X.M. and Song, X.T. (2009). The new trend of Chinese farmer's cooperative organisations after the China Cooperative Law's enactment [in Chinese]. China Agricultural Economy [online resource] Retrieved on 15th June 2010 via http://hezuoshe.banzhu.net/article/hezuoshe-3-412769.html

H

Hahn, T., Olsson, P., Folke, C., Johansson, K., (2006). Trust-building, knowledge generation and organizational innovations: the role of a bridging organization for adaptive comanagement of a wetland landscape around Kristianstad, Sweden. Human Ecology 34:573-592.

Han, J. (2009). To improve the working environment for rural workers in town [in Chinese]. Accessed on 14th May 2011 via http://www.china.com.cn/chinese/zhuanti/jd/497089.htm.

Hanazaki, N., Tamashiro, J., Leitao-Filho, H. and Begossi, A. (2000). Diversity of plant uses in two Caiçara communities from the Atlantic Forest coast, Brazil. Biodiversity and Conservation 9:597-615.

Hanna, S., Folke, C., and Maler, K. G. (1996). Rights to Nature. Washington, D.C., USA: Island Press.

Hao, P.N. (1962). How the settlement policy has been implemented in Inner Mongolia. [in Chinese]. Chinese Minorities 21: 35-45.

Hardin, G. (1968). The tragedy of the commons. Science 162 (3859): 1243-1248.

Hardin, G., and Baden, J. (Eds.). (1977). Managing the Commons. San Francisco: W.H. Freeman and Company. 
Harris, G. (2007). Seeking Sustainability in an Age of Complexity. Cambridge, UK: Cambridge University Press.

Harrison, K. D. (2007a). An extinction of (ideas about) species. In When Languages Die: The Extinction of the World's Languages and the Erosion of Human Knowledge (pp. 23-59). Oxford: Oxford University Press.

Harrison, K. D. (2007b). A world of Many (fewer) voices. In When Languages Die: The Extinction of the World's Languages and the Erosion of Human Knowledge (pp. 121). Oxford: Oxford University Press.

Hasi, B.G., Ye,X.H. and Zhao,H. (2011). The Research of Traditional Ecological knowledge of Mongolian Wild Edible Plants in Xilin'guole Grassland [in Chinese]. Plant Taxonomy and Resources 33 (2): 22-27.

Hay, I. (2005). Qualitative Research Methods in Human Geography. Oxford, UK: Oxford University Press.

Hays, J. S. (2009). A step forward and new challenges: indigenous communities and mother-tongue education in southern Africa. International Journal of Bilingual Education and Bilingualism, 12(4): 401-413.

Headland, T., Pike, K. and Harrism M. (Eds.) (1990). Emics and Etics: The Insider/Outsider Debate. London: Sage Publications.

Heckler, S. (2007). On knowing and not knowing: The many valuations of Piaroa local knowledge. In P. Sillitoe (Ed.), Local science vs. global science: Approaches to indigenous knowledge in international development. New York: Berghahn Books.

Heinrich, M., Ankli, A., Frei, B., Weimann, C. and Sticher, O. (1998). Medicinal plants in Mexico: healers' consensus and cultural importante. Social Science and Medicine 47:1859-1871.

Hickey F.R. and Johannes R.E. (2002). Recent evolution of village-based marine resource management in Vanuatu. SPC Traditional Marine Resource Management and Knowledge Information Bulletin 14:8-21. Available online at www.spc.int/coastfish/News/Trad/trad.htm

Hilborn, R. and Gunderson, D. (1996). Chaos and paradigms for fisheries management. Marine Policy 20: 87-89.

Hill, H. J. (2004). What is lost when names are forgotten? In G. Sanga \& G. Ortallis (Eds.), Nature Knowledge, Ethnoscience, Cognition, and Utility (pp. 161-184). New York: Berhahm Books.

Hinch, T. (1998). Ecotourists and indigenous hosts: Diverging views on their relationship with nature. Current Issues in Tourism 1(1): 120-124. 
Hoadley, S. (1992). The South Pacific foreign affairs handbook. Sydney, Australia: Allen \& Unwin in association with the New Zealand Institute of International Affairs

Hodgson, D.L. (2002).Introduction: Comparative Perspectives on the Indigenous Rights Movement in Africa and the Americas. American Anthropologist 104 (4): 10371049.

Holling, C. S. (Ed.) (1978). Adaptive Environmental Assessment and Management. London: Wiley.

Holling, C.S. and Meffe, G.K. (1996). Command and control and the pathology of natural resource management. Conservation Biology 10: 328-37.

Holling, C.S., Schindler, D.W., Walker, B.W. and Roughgarden, J. (1995). Biodiversity in the functioning of ecosystems: An ecological synthsis. In C. Perrings, K-G. Maler, C. Folke., C.S. Holling, and B.O. Jansson. (Eds.), Biodiversity Loss (pp.44-83). Cambridge: Cambridge University Press.

Holt, F.L. (2005) The catch-22 of conservation: indigenous peoples, biologists and cultural change. Human Ecology 33: 199-215.

Houde, N. (2007). The six faces of traditional ecological knowledge: challenges and opportunities for Canadian co-management arrangements. Ecology and Society 12(2): 34. [online] URL: http://www.ecologyandsociety.org/vol12/iss2/art34/

Hviding, E. (2006). Knowing and managing biodiversity in the Pacific Islands: challenges of environmentalism in Marovo Lagoon. International Social Science Journal 187: 69-85.

$\mathrm{Hu}, \mathrm{J} . \mathrm{T}$. (2005). The public speech at the five year anniversary of the Grant Western Development Strategy by Hu Jintao [in Chinese]. Accessed on $5^{\text {th }}$ September 2010 via http://www.gov.cn/ldhd/2005-02/05/content_9525.htm.

Hu, X.H. (2009). The Study of Mongolian Ethnobotanical Knowledge in Xilin'guole Grassland, Inner Mongolia [in Chinese]. Masters thesis submitted to Inner Mongolia Normal University, China.

Huertas, C.B. (2004). Indigenous Peoples in Isolation in the Peruvian Amazon. IWGIA Document No. 100. Copenhagen: IWGIA.

Huggett, A. J. (2005). The concept and utility of 'ecological thresholds' in biodiversity conservation. Biological Conservation 124 (3): 301-310.

Hunn, E. (1993). What is traditional ecological knowledge? In N.M. William, and G. Baines (Eds), Traditional Ecological Knowledge: Wisdom for Sustainable Developemnt (pp.13-15). Canberra: Centre for Resource and Environmental Studies, Australia National University. 
Hurrell, A. (2005). Power, institutions and the production of inequality. In M. Barnett, and R. Duvall (Eds.). Power in global governance (pp. 33-58). Cambridge: Cambridge University Press.

\section{I}

Iaarossi, G. (2006). The Power of Survey Design: A User's Guide for Managing Surveys, Interpreting Results, and Influencing Respondents. Washington, D.C.: World Bank.

Iain, H. (2005). Qualitative Research Methods in Human Geography. Oxford: Oxford University Press.

IIED (International Institute of Environment and Development). (2006). Protecting Community Rights over Traditional Knowledge: Implications of customary laws and practices: Interim Report 2005-2006. London: IIED.

IIED (International Institute of Environment and Development). (2009). Protecting community rights over traditional knowledge: Implications of customary laws and practices - Key findings and recommendations 2005-2009. London: IIED.

ILO (International Labour Organisation). (1989). International Labour Organisation Convention No. 169. Can be accessed via http://www.ilo.org/indigenous/Conventions/no169/lang--en/index.htm

Inglis, J.T. (Ed.) (1993). Traditional Ecological Knowledge - Concepts and Cases. Ottawa: International Program on Traditional Ecological Knowledge \& International Development Centre.

Inshigawa, J. (2006). Cosmovisions and environmental governance. The case of in situ conservation of native cultivated plants and their wild relatives in Peru. In W.V. Reid, F. Berkes, T. Wilbanks and D. Capistrano (Eds), Bridging Scales and Knowledge Systems (pp. 207-224). Washington, DC: Island Press.

International Society of Ethnobiology. (2006). The Code of Ethics. Can be accessed at http://ethnobiology.net/code-of-ethics/code-in-english/

Iskandar, J. (2007). Responses to environmental stree in the Baduy swidden system, south Banten, Java. In R. Ellen (Ed.) Modern Crises and Traditional Strategies: Local ecological knowledge in Islands Southeast Asia (pp84-111). New York: Berghahn Books.

Ito, S. (2004). Globalisation and agrarian change: A case of freshwater prawn farming in Bangladesh. Journal of International Development 16(7): 1003-1013.

IUCN-ICTFIP (International Union for the Conservation of Nature, Inter-Commission Task Force on Indigenous Peoples). (1997). Indigenous peoples and sustainability : Cases and actions. Gland, Switzerland: IUCN. 
IUCN (World Conservation Union). (2005a). Threats authority file. Version 2.1. IUCN Species Survival Commission, Cambridge, United Kingdom. Available from http://iucn.org/webfiles/doc/SSC/RedList/AuthorityF/threats.rtf (accessed October 2007).

IUCN (World Conservation Union). (2005b). Conservation actions authority file. Version 1.0. IUCN Species Survival Commission, Cambridge, United Kingdom. Available from http://iucn.org/webfiles/doc/SSC/RedList/AuthorityF/consactions.rtf (accessed October 2007).

IUCN (World Conservation Union). (2006). Habitats authority file. Version 1.0. IUCN Species Survival Commission, Cambridge, United Kingdom. Available from http://iucn.org/webfiles/doc/SSC/RedList/AuthorityF/habitats.rtf (accessed October 2007).

\section{J}

Jacobs, A.H. (1980). Pastoral Maasai and tropical rural development. In R.H. Bates and M.F. Lofchie (Eds.), Agricultural Development in Africa: Issues of Public Policy (pp. 275-300). New York: Praeger.

Jiang, G.M., Han, X.G., and Wu, J.G. (2006). Restoration and management of the Inner Mongolia grassland require a sustainable strategy. AMBIO (Journal of Human Environment) 35(5): 269-70.

Jimu, S., Bao, X.X., Bude, B.T. and Aote, G.T.Y. (2009). The effects of different herding methods on Yunake's stipa grandis steppe communities on the Mongolian stepper [in Chinese and Mongolian]. The collection of the proceeding papers of the Forum of China and Mongolia Cooperative Projects 2009: Ulan Bator.

Jin, X.L. (2002). A review of the legislations since the economic reform [in Chinese]. Journal of Politics and Legislations 4: 23-27.

Jodha, N.S. (1998) Poverty and environmental resource degradation: An alternative explanation and possible solutions. Economic and Political Weekly 5(12): 2384-2390.

Johannes, R. E. (1978). Traditional marine conservation methods in Oceania and their demise. Annual Review of Ecology and Systematics 9:349-364.

Johannes, R. E., (Ed.) (1989). Traditional ecological knowledge: A collection of essays. International Conservation Union (IUCN), Gland, Switzerland.

Johannes, R. E. (1998). The case for data-less marine resource management: examples from tropical nearshore fisheries. Trends in Ecology and Evolution 13:243-246.

Johnson, A.W. (1978). Quantification in Cultural Anthropology. Stanford, CA.: Stanford University Press. 
Johnson, L.M. (2006). Gitksan medicinal plants-cultural choice and efficacy. Journal of Ethnobiology and Ethnomedicine 2:29. URL

http://www.biomedcentral.com/1746-4269/2/29

Jones, H. (2011). A guide to monitoring and evaluating policy influences. Background Note for Overseas Development Institute.

$\mathbf{K}$

Kanu, Y. (2006). Curriculum as Cultural practice: Postcolonial Imaginations. Toronto: University of Toronto Press Inc.

Kastrup, J.P. (1997). The internationalisation of indigenous rights from the environmental and human rights perspective. Texas International Law Journal, $32: 97-122$.

Kater, A. (1993). Indigenous learning in crafts: A pilot research effort. Indigenous Knowledge and Development Monitor 1(1): 20-22.

Keith, R.F. and Simon, M. (1987). Sustainable development in the northern circumpolar world. In P. Jacobs and D.A. Munro (Eds.), Conservation with equity (pp. 209-225). Cambridge: IUCN.

Kellert, S. R., Mehta, J. N., Ebbin, S. A., and Lichtenfeld, L. L. (2000). Community natural resource management: promise, rhetoric, and reality. Society \& Natural Resources 13(8): 705-715.

Kendie, S.B. and Guri, B.Y. (2006). Indigenous institutions as parameters for agriculture and natural resource management. In D. Miller, S.B. Kendie, A. Apusiga and B. Haverkort (eds), African Knowledge and Sciences: A Potential for Endogenous Development (pp.332-349). COMPAS Series on Worldviews and Sciences 3. Holland: COMPAS ETC Publication.

Kenrick, J. (2000). The Forest Peoples of Africa in the 21st Century. Indigenous Affairs, Hunters and Gatherers 2000(2): 10-24.

King, C. (2012). Building capacity to engage: community engagement or government engagement? Community Development Journal 47 (1): 5-28.

King, C. and Cruickshank, M. (2012). Building capacity to engage: Community engagement or government engagement? Community Development Journal 47(1):5-28.

Kilduff, M. and Tsai, W. (2003). Social networks and organisations. London: Sage.

Kipuri, N. (2009). Chapter II: Culture. In DESA of UN, State of World's Indigenous Peoples (pp52-81). United Nations Document. New York, USA. 
Kirsch, S. (2001). Lost words: environmental disaster, "cultural loss", and the law. Cultural Anthropology 42: 167-198.

Kitzinger, J. and Barbour, R. S. (1999). Developing focus group research: politics, theory and practice. IONDON: SAGE.

Kolff, J. (1965). The economic implications of Self-Government for the Cook Islands. Journal of the Polynesian Society 74 (1): 114-124.

Kooiman, J. (2003). Governing the Governance. London: Sage Publication.

Kooiman, J., Bavinck, M., Jentoft, S. and Pulin, R. (eds) (2005). Fish for Life: Interactive Governance for Fisheries. Amsterdam, the Netherlands: Amsterdam University.

Koontz, T. M. and Thomas, C.W. (2006). What do we know and need to know about the environmental outcomes of collaborative management? Public Administrative Review 66:111-122.

Krauss, M. (1992). The World's Languages in Crisis. Language 68(1):4-10.

Krauss, M., Maffi, L. and Yamamoto, A. (2004). The world's languages in crisis: Questions, challenges, and a call for action. Lectures on Endangered Languages.

Kremen, C., Raymond, I. and Lance, K. (1998). An Interdisciplinary Tool for Monitoring Conservation Impacts in Madagascar. Conservation Biology 12:549-563.

Kristensen, M. and Balslev, H. (2003). Perceptions, use and availability of woody plants among the Gourounsi in Burkina Faso. Biodiversity and Conservation 12(8): 17151739.

Kumar, S. (2006). Methods for Community Participation: A Complete Guide for Practitioners. UK: YIDG Publishing.

$\mathbf{L}$

Labonte, R. (1999). Social capital and community development: Practitioner emptor. Australia and New Zealand Journal of Public Health 23 (4): 430-433.

Lacity, M. C. and Jason, M. A. (1994). Understanding Qualitative Data: A Framework of Text Analysis Methods. Journal of Management Information Systems 11(2): 137155.

Ladefoged, P. (1992). Another view of endangered languages. Language 68(4): 809-811.

Lai, C.T. (2003). Daoism in China Today, 1980-2002. The China Quarterly 174:413-427. 
Lalonde, A. (1993). African indigenous knowledge and its relevance to sustainable develoment. In J. Inglis (Ed.), Traditional Ecological Knowledge: Concepts and Cases (pp. 55-62). Ottawa: International Program on Traditional Ecological Knowledge \& International Development Research Centre.

Lane, C. and Moorehead, R. (1993). New Direction in African Range Management, Natural Resource Tenure and Policy. London: ODI/IIED.

Larson, A.M. and Soto, F. (2008). Decentralisation of natural resource governance regimes. Annual Review of Environment and Resources 33: 213-239.

Lasimbang, J. (2008). Indigenous peoples and local economic development. Local Glob 2008 (5): 42-45.

Lawrey, A. (1990). Contemporary Efforts to Guarantee Indigenous Rights under International Law. Vanderbilt Journal of Transnational Law 703:703 -778.

Leach, M., Mearns, R., and Scoones, I. (1999). Environmental Entitlement: Dynamics and Institutions in Community-based Natural Resources Management. World Development 27(2): 225-247.

Leclair, M.S. (2008). Fighting the tide: Alternative trade organisations in the era of global free trade - A reply. World Development 36(12): 2962-2965.

Lee, K. N. (1993). Compass and Gyroscope. WWashington, D.C.: Island Press.

Lee, K. N. (1999). Appraising adaptive management. Conservation Ecology 3(2): 3. [online] URL: http://www.consecol.org/vol3/iss2/art3/

Lemos, M. C. and Agrawal, A. (2006). Environmental governance. Annual Review of Environment and Resources, 31, 297-325.

Lepage, P. (1994). Indigenous peoples and the evolution of international standards: A shaort history. In M. Leger (Ed.), Aboriginal Peoples: Towards Self-government ( $p p$. 1-24). Montreal, Canada: Black Rose Books.

Levi-Strauss, C. (1962). La pensee sauvage. Librarie Plon, Paris, France. English translation, 1966. The savage mind. Chicago: University of Chicago Press.

Levin, S. A. (1998) Ecosystems and the biosphere as complex adaptive systems. Ecosystems 1: 431-436.

Lewis, H. T., and Ferguson, T. A. (1988). Yards, corridors, and mosaics: how to burn a boreal forest. Human Ecology 16: 57-77.

Li, W.J., Ali, S.H. and Zhan, Q. (2007). Property rights and grassland degradation: A study of the Xilingol pasture, Inner Mongolia, China. Journal of Environmental Management 85(2): 461-70. 
Linberg, D. C. (2007). The beginnings of Western science: The European scientific tradition in philosophical, religious, and institutional context, 600 BC to AD 1450. Chicago: University of Chicago Press.

Liu, J.G., Dietz, T., Carpenter, S.R., Alberti, M., Folke, C., Moran, E., Pell, A.N., Deadman, P., Kratz, T., Lubchenco, J., Ostrom, E., Ouyang, Z., Provencher, W., Redman, C.L., Schneider, S.H. and Taylor, W.W. (2007). Complexity of coupled human and natural systems. Science 317: 1513-1516.

Liu, S.R., Ji, M.S. and Bao, X.X. (2009). The degree of herding activities and its effects on vegetation in Mongolian grassland [in Chinese and Mongolian]. The collection of the proceeding papers of the Forum of China and Mongolia Cooperative Projects 2009: Ulan Bator.

Liu, Y. C. (1998). History and the present, Han and Mongolian in Alashan League: A research of relationship between different ethnic groups [in Chinese]. Northwest Ethnography Studies 1: 65-76.

Lizarralde, M. (1997). Perception, knowledge and use of the rainforest: Ethnobotany of the Bari of Venezuela. PhD dissertation. University of California at Berkeley.

Lizarralde, M. (2001). Biodiversity and loss of indigenous languages and knowledge. In L. Maffi (Ed.), On Biocultural Diversity: Linking Language, Knowledge, and the Environment (pp. 265-281). Washington, DC: Smithson. Inst. Press.

Lizarralde, M. (2004). Indigenous Knowledge and Conservation of the Rain Forest: Ethnobotany of the Barí of Venezuela. Advances in Economic Botany 15:113-131.

Logan, B.I. and Moseley, W.G. (2002). The political ecology of poverty alleviation in Zimbabwe's Communal Areas Management Programme for Indigenous Resources (CAMPFIRE). Geoforum 33(1): 1-14.

Lykke, A.M., Kristensen, M.K. and Ganaba, S. (2004). Valuation of local use and dynamics of 56 woody species in the Sahel. Biodiversity and Conservation 13:1961-90.

M

Maffi, L. (Ed.) (2001). On Biocultural Diversity: Linking Language, Knowledge, and the Environment. Washington, D.C.: Smithsonian Press.

Maffi, L. (2005a) Linguistic, cultural, and biological diversity. Annual Review of Anthropology 34:599-617.

Maffi, L. (2005b). Section on Cultural Indicators. In Interim Report to the World Bank on socioeconomic, cultural, governance, and human health indicators of the health of the Mesoamerican Reef Region. 
Mannion, J. (1996). Partnership, participation and capacity building: rural development based on local bottom-up strategies. Leader Magazine 12 (10): Article 2.

Manor, J. (1999). The Political Economy of Decentralisation. Washington, D.C: World Bank.

Marcus, C. (2002). Indigenous Rights and the Collective Conscious. Anthropology Today 18(1):1-3.

Martin, D. (1995). Money, business and culture: issues for Aboriginal economic policy. CAEPR Discussion Paper No. 101. Canberra: Centre for Aboriginal Economic Policy Research, the Australian National University.

Mathur, A. (2003). Who owns traditional knowledge? Working paper No.96. Indian Council for Research on International Economic Relations. New Delhi.

Mauro, F. and Hardison, P. D. (2000). Traditional knowledge of indigenous and local communities: International debate and policy initiatives. Ecological Applications 10:1263-1269.

May, S. (1999). Language and education rights for indigenous people. In S. May, (Ed.) Indigenous Community-based Education ( $p p$ 42-66). PA: Multilingual Matters Ltd.

Mazzocchi, F. (2006). Western science and traditional knowledge: Despite their variations, different forms of knowledge can learn from each other. EMBO reports 7(5):463-465.

McCarter, J. (2012). Variation, transmission, and maintenance of traditional ecological knowledge on Makula Island, Vanuatu. A PhD thesis submitted to University of Wellington, New Zealand.

McCarty, T. and Lucille, J. W. (1999). Indigenous community-based language education in the USA. In S. May (Ed.), Indigenous Community-based Education (pp 79-94). PA: Multilingual Matters Ltd.

McCarty, N., Swallow, B., Kirk, M and Hazell, P. (2000). Property Rights, Risk, and Livestock Development in Africa. Washington, D.C.: International Food Policy Research Institute.

McGivney, V. (1999). Informal Learning in the Community: A Trigger for Change and Development. Leicester: National Institute for Adualt Continuing Education.

McGregor, D. (2004). Coming full circle: indigenous knowledge, environment, and our future. American Indian Quarterly 28(3 \& 4): 385-410.

McIntosh, A.J. and Ryan, C. (2007). The market perspective of indigenous tourism: Opportunities for business development. In R. Butler and T. Hinch (Eds.), Tourism and Indigenous Peoples: Issues and Implications (pp. 72-83). Oxford: Elsevier Ltd. 
McIntyre, K and Gosford, L. (2011). Sustainable futures: Indigenous tourism. International Trade Forum 2011(2):35.

McManis, C. (2007). Biodiversity and the Law: Intellectual Property, Biotechnology and Traditional Knowledge. London: Routledge.

Mead, T. P. A. (2005). Emerging issues in Maori traditional knowledge: Can these be addresses by United Nations agencies? Paper presented at the UNPFII Workshop, Panama, September 2005.

Meadowcroft, J. (1993). Review of Robyn Eckersley 'Environmentalism and Political Theory: Toward an Eco-centric Approach'. Canadian Journal of Political Science 26:420-421.

Medley, K. E. and Kalibo, H. W. (2005). An Ecological Framework for Participatory Ethnobotanical Research at Mt. Kasigau, Kenya. Field Methods 17(3):302-14.

Michael, A. (1981). Building United Judgement: A Handbook for Consensus Decision Making. Madison: The Centre for Conflict Resolution.

Migot-Adholla, S., Hazell, P., Blarel, B. and Place, F. (1991). Indigenous land rights systems in Sub-Saharan Africa: A constraint on productivitie? The World Bank Economic Review 5(1):155-175.

Miller, J. R. (1987). The irony of residential schooling. Canadian Journal of Native Education 14(2): 3-14.

Miller, M.L., Kaneko, J. Bartram, P., Marks, J. and Brewer, D.D. (2004) Cultural consensus analysis and environmental anthropology: Yellowfin tuna fishery management in Hawaii. Cross-Cultural Research 38:289-314.

Milton, K. (2001). Aspects and Implications of Ecological Diversity in Forest Societies of the Brazilian Amazon. In L. Maffi (Ed.), On Biocultural Diversity: Linking Language, Knowledge, and the Environment (pp. 282-297). Washington, DC: Smithson. Inst. Press.

Minol, B. (2000). Manus from the legends to year 2000: A history of the people of Manus. Port Moresby, Papua New Guinea: UPNG Press.

Moller, H. (2009a). Guidelines for cross-cultural participatory action research partnerships: A case study of a customary seabird harvest in New Zealand. New Zealand Journal of Zoology. 36:211-41.

Moller, H. (2009b). Matauranga Maori, science and seabirds in New Zealand. New Zealand Journal of Zoology. 36: 203-10.

Morrison, B. (2007). Co-management: Case studies involving local authorities and Maori. Accessed on $6^{\text {th }}$ September 2011 via 
http://www.lgnz.co.nz/library/files/store 016/Co-managementCaseStudies InvolvingLocalAuthoritiesAndMaoriJanuary2007.pdf

Morris, M. W., Leung, K., Ames, D. and Lickel, B. (1999). Views from inside and outside: Integrating Emic and Etic Insights about Culture and Justice Judgment. The Academy of Management Review 24(4): 781-796.

Mowbray, M. (2005) Community capacity building or state opportunism? Community Development Journal 40 (3): 255-264.

$\mathrm{Mu}, \mathrm{L}$. (2007). The Comparative Study of Wild Traditional Chinese and Mongolian Medicinal Plants in Inner Mongolia [in Chinese]. Masters thesis submitted to Inner Mongolia Normal University, China.

Mugabe, J. (2000). Intellectual property protection and traditional knowledge: An explanation in international policy discourse. Working paper. African Center for Technology Studies. Nairobi, Kenya.

Mukundi, G. W. (2009). Kenya: Constitutional, Legislative and Administrative Provisions Concerning Indigenous Peoples. International Labour Organisation and Africa Commission on Human Rights \& Peoples' Rights Doc.

Mulligan, M. (2003). Feet to the ground in storied landscapes: disrupting the colonial legacy with a poetic politics. In W. M. Adams and M. Mulligan. (Eds.), Decolonising nature: Strategies for conservation in a Post-colonial era. (pp. 268-289). London: Earthscan.

Murdoch, J. and Abram, S. (1998) Defining the limits of community governance. Journal of Rural Studies 14 (1): 41-50.

Myer, L. (2000). Biodiversity hotspots for conservation priorities. Nature 403: 853-858.

$\mathbf{N}$

Nabhan, G. P. and St Antoine, S. (1993). The loss of floral and faunal story: The extinction of experience. . In S. R. Kellert \& E. O. Wilson (Eds.), The Biophilia Hypothesis (pp. 229-250). Washington D.C.: Island Press.

Nabhan, G.P. (2000). Native American management and conservation of biodiversity in the Sonoran Desert bioregion. In P.E. Minnis and W. J. Elisens (Eds), Biodiversity and Native America (pp. 29-43). Norman OK: University of Oklahoma Press.

Nadasdy, P. (1999). The politics of TEK: Power and the 'integration' of knowledge. Arctic Anthropology 36(1/2), 1-18.

Nadasdy, P. (2005). Transcending the debate of the ecologically noble Indian: Indigenous peoples and environmentalism. Ethnohistory 52(2): 291-331. 
Nakashima, D. J. (1993). Astute observers on the sea ice edge: Inuit knowledge as a basis for Arctic co-management. In J. Inglis (Ed.), Traditional Ecological Knowledge: Concepts and Cases (pp. 99-110). Ottawa: International Program on Traditional Ecological Knowledge \& International Development Research Centre.

Nakashima, D.J. (1998). Conceptualising nature, the cultural context of resource management. Nature and Resources 34 (2):8-22.

Natcher, D.C., Davis, S. and Hickey, C.G. (2005). Co-management :relationships, not resources. Human Organisation 64:240-250.

Natcher, D.C. and Davis, S. (2007). Rethinking Devolution: Challenges for Aboriginal Resource Management in the Yukon Territory. Society \& Natural Resources 20(3): 271-279.

Nelson, M.C., Kintigh, K., Abbott, D.R. and Anderies, J.M. (2010). The cross-scale interplay between social and biophysical context and the vulnerability of irrigationdependent societies: Archaeology's long-term perspective. Ecology and Society 15(3). Article 31. URL: http://www.ecologyandsociety.org/vol15/iss3/art31/

Nettle, D. and Romaine, S. (2000). Vanishing Voices: the extinction of the world's languages. Oxford: Oxford University Press.

Niamir, M. (Ed.) (1994). Proceedings of Workshop on Pastoral Natural Resource Management and Pastoral Policy in Africa, Arusha, Tanzania. New York: United Nations Sudano-Sahelian Office.

Niamir, M. (1998). The resilience of pastoral herding in Sahelian Africa. In F.Berkes and C. Folke (Eds.), Linking Social and Ecological System: Management Practices and Social mechanisms for Building Resilience (pp. 250-284). Cambridge, UK: Cambridge University Press.

Nie, A.W. (2007). The settlement of nomadic herders and their future. [in Chinese]. Qinghai Minority Research 1: 56-66.

Niroa, J. (2004). Why we need to re-think Vanuatu education. In K. Sanga, J. Niroa, K. Matai \& L. Crowl (Eds.), Re-thining Vanuatu education together. Port Vila: University of the South Pacific.

Niu, C. H., Hu, C. Y., Hao, J., Niu, L. Z., and Zhang, C. X. (2002). Control of Rhombomys opimus in Haloxylon ammodendron Bunge forests in Inner Mongolia [in Chinese]. Forestry Science and Technology 6: 15-18.

Inner Mongolia Bureau of Environment Protection. (2007). The Annual Report of the Environment in Inner Mongolia [in Chinese]. Accessed on $6^{\text {th }}$ May 2011 via http://www.nmgepb.gov.cn/ 
Noiriel, G. (2006). Colonialism, immigration, and power relations. Qualitative Sociology 29(1): 105-110.

North, D. C. (1990). Institutions, Institutional Change and Economic Performance. Cambridge: Cambridge University Press.

North, D.C. (1991). Institutions. The Journal of Economic Perspectives. 5(1): 97-112.

NRC (National Research Council of USA). (2002). The Drama of the Commons. Committee on Human Dimensions of Global Change. National Academy Press: Washington, DC.

\section{$\mathbf{0}$}

O’Brien, K., Hayward, B. and Berkes, F. (2009) Rethinking social contracts: building resilience in a changing climate. Ecology and Society 14(2): Article 12. [online] http://www.ecologyandsociety.org/vol14/iss2/art12/

Ohmagari, K. and Berkes, F. (1997). Transmission of indigenous knowledge and bush skills among the Western James Bay Cree women of sub Arctic Canada. Human Ecology 25(2):197-221.

Olsen, C.S. and Helles, F. (1997). Medicinal plants, markets, and margins in the Nepal Himalaya: trouble in paradise. Mountain Research and Development 17:363-374.

Olsson, P. and Folke, C. (2001). Local ecological knowledge and institutional dynamics for ecosystem management: A study of Lake Racken Watershed, Sweden. Ecosystems 4(2): 85-104.

Ostrom, E. (1990). Governing the Commons: The Evolution of Institutions for Collective Actions. Cambridge: Cambridge University Press.

Ostrom, E. (2005). Understand Institutional Diversity. Princeton: Princeton University Press.

Ostrom, E. (2007a). Diagnostic approach for going beyond panaceas. Proceedings of the National Academy of Sciences 104: 5181-5187.

Ostrom, E. (2007b). Sustainable social-ecological systems: An impossibility? Workshop in Political Theory and Policy Analysis. Indiana University.

Ostrom, E. (2009). A general framework for analysing sustainability of social-ecological systems. Science 325(5939):419-422.

Ostrom, E. and Janssen, A. (2004). Multi-level governance and resilience of socialecological systems. In M. Spoor (Ed), Globalisation, Poverty and Conflicts (pp. 239259). Netherland: Kluwer Academic Publisher. 
Ostrom, E., Burger, J., Field, C. B., Norgaard, R. and Policansky, D. (1999). Revisiting the commons: Local lessons, global challenges. Science 284 (5412): 278-282.

Ostrom, E., Gardner, R. and Walker, J. (Eds.). (1997). Rules,Games, and Common-Pool Resources. Michigan: The University of Michigan Press.

$\mathbf{P}$

Pagdee, A., Kim, Y. S. and Daugherty, P.J. (2006). What makes community forest management successful: A meta-study from community forests throughout the world. Society and Natural Resources 19:33-52.

Pahl-Wostl, C. (2009). A conceptual framework for analyzing adaptive capacity and multi-level learning processes in resource governance regimes. Global Environmental Change 19: 354-365.

Pan, J. J., Zhang, S. H., Wang, X. Q., Hao, J., and Ao, T. S. (2005). Experiment on aerial control of rat damage in desert Haloxylon ammodendron forest in Alashan league, Inner Mongolia [in Chinese]. Inner Mongolia Forestry Science and Technology 3: 7781.

Pasqualucci, J. M. (2006). Evolution of International Indigenous Rights in the InterAmerican Human Rights System, Human. Rights Law Review 281: 281-322.

Perrings, C. and Walker, B.W. (1995). Biodiversity loss and the economics of discontinuous change in semiarid rangeland. In C. Perrings, K.G. Maler, C. Folke, C.S. Holling and B.O. Jansson (Eds.) Biodiversity Loss: Economic and Ecological Issues (pp.190-210). Cambridge: Cambridge University Press.

Phillips, O., and Gentry, A.H. (1993a). The Useful Plants of Tambopata, Peru: I. Statistical Hypothesis Tests with a New Quantitative Technique. Economic Botany 47(1):1532.

Phillips, O., and Gentry, A.H. (1993b). The Useful Plants of Tambopata, Peru: II. Additional Hypothesis Testing in Quantitative Ethnobotany. Economic Botany 47(1):33-43.

Phillips, O., Gentry, A.H., Reynel, C., Wilkin, P. and Gálvez-Durand, C. (1994). Quantitative Ethnobotany and Amazonian Conservation. Conservation Biology 8:225-248.

Pinkerton, E. (1998). Integrated management of a temperate montane forest ecosystem through wholistic forestry: A British Columbia example. In F.Berkes \& C. Folke (Eds.), Linking Social and Ecological System: Management Practices and Social mechanisms for Building Resilience (pp. 363-389). Cambridge, UK: Cambridge University Press.

Pomeroy, R.S. and Berkes, F. (1997). Two to tango: the role of government in fisheries comanagement. Marine Policy 21: 465-480. 
Popham, J. (1996). Empowerment through community enterprise. Local Government Policy Making 23 (2): 26-30.

Posey, D.A. (1990). Intellectual property rights and just compensation for indigenous peoples. Anthropology Today 6(4): 13-16.

Posey, D. A. (Ed.) (1999). Cultural and Spiritual Values of Biodiversity. Intermediate Technology Publications, London.

Posey, D. (2000). Ethnobiology and ethnoecology in the context of national laws and international agreements affecting indigenous and local knowledge, traditional resources and intellectual property rights. In R. Ellen, P. Parkes \& A. Bicker (Eds.), Indigenous Environmental Knowledge and its Transformations: Critical Anthropological Perspectives. London and New York: Routledge.

Posey, D. (2001). Biological and cultural diversity: The inextricable, linked by language and politics. In L. Maffi (Ed.), On Biocultural Diversity: Linking Language, Knowledge and the Environment. Washington DC: Smithsonian Institution Press.

Posey, D.A. (2004). The 'balance sheet' and the 'sacred balance': Valuing the knowledge of indigenous and traditional peoples. In Posey, D. and K. Plenderleith, (Ed.) Indigenous Knowledge and ethics: A Darrell Posey Reader (pp. 195-205) New York: Routledge.

Posey, D. and Dutfield, G. (1996). Beyond intellectual property: Toward traditional resource rights for indigenous peoples and local communities. Ottawa: International Development Research Centre.

Posey, D. and Dutfield, G. (1997). Indigenous People and Sustainability: Cases and Actions. Utrecht: International Books/ IUCN.

Posey, D. A. and Balick, M.J. . (2006). Human Impacts on Amazonia: The Role of Traditional Ecological Knowledge in Conservation and Development. New York: Columbia University Press.

Pressey, R. L. and Bottrill, M. C. (2008). Opportunism, threats, and the evolution of systematic conservation planning. Conservation Biology 22(5): 1340-1345.

Preston, R.J. (2002). Cree narrative: Expressing the personal meaning of event. Montreal: McGill-Queen's University Press.

Prince, R.J., Geissler, P.W., Nokes, K., Maende, J.O., Okatcha, F. and Sternberg, R. (2001). Knowledge of herbal and pharmaceutical medicines among Luo children in western Kenya. Anthropology and Medicine 8:211-235.

Prusha, F. P. (1994). American Indian Treaties: The History of a Political Anomaly. Berkeley, California, USA: California University Press. 
Punch, K. F. (2005). Introduction to social research: quantitative and qualitative approaches. London: SAGE.

Q

Quinlan, M. B. and Quinlan, R. J. (2007). Modernization and Medicinal plant knowledge in a Caribbean Horticultural village. Medical Anthropology Quarterly 21(2): 169192.

$\mathbf{R}$

Ramirez, C. R. (2007). Ethnobotany and the Loss of Traditional Knowledge in the 21st Century. Ethnobotany Research \& Applications 5:245-247.

Rasmussen, L. N. and Meinzen-Dick, R. (1995). Local organisations for natural resource management: Lessons from theoretical and empirical literature. Paper presented at the Local Organisations for Natural Resource Management.

Redford, K. and Sanderson, S. (2000) Extracting humans from nature. Conservation Biology 14: 1362-1364.

Ray, B. (2005). Creating a Place for Indigenous Knowledge in Education: The Alaska Native Knowledge Network. Accessed on $5^{\text {th }}$ May 2011 via http://www.ankn.uaf.edu/curriculum/articles/raybarnhardt/pbe_ankn_chapt er.html

Raza, A., Rashid Kausar, A. and Paul, D. (2007) The social management of embodied knowledge in a knowledge community. Journal of Knowledge Management 11(5): $45-54$.

Reyes-García, V. (2001). Indigenous People, Ethnobotanical Knowledge, and Market Economy: A Case Study of the Tsimane' Amerindians in Lowland Bolivia. PhD Dissertation, University of Florida. Ann Arbor: UMI.

Reyes-Garcia, V., Godoy, R., Vadez, V., Apaza, L., Byron, E., Huanca, T., Leonard, W.R., Pérez E. and Wilkie, D. (2003). Ethnobotanical Knowledge Shared Widely among Tsimane Amerindians, Bolivia. Science 299:1707.

Reyes-García, V., Byron, E., Vadez, V., Godoy, R., Apaza, L., Pérez Limache, E., Leonard, W.R. and Wilkie, D. (2004). Measuring Culture as Shared Knowledge: Do Data Collection Formats Matter? Cultural Knowledge of Plant Uses Among Tsimane' Amerindians, Bolivia. Field Methods 16(2):135-156.

Reyes-Garcia, V., Vadez, V., Huanca, T., Leonard, W.R. and Wilkie, D. (2005a). Knowledge and consumption of wild plants: A comparative study in two Tsimane' villages in the Bolivian Amazon. Ethnobotanical Research Applications 3:201-207. 
Reyes-Garcia, V., Vadez, V., Byron, E., Apaza, L., Leonard, W.R., Pérez, E. and Wilkie, D. (2005b). Market Economy and the Loss of Folk Knowledge of Plant Uses: Estimates from the Tsimane ' of the Bolivian Amazon. Current Anthropology 46(4):651-656.

Reyes-Garcia, V., Huanca, T., Vadez, V., Leonard, W.R. and Wilkie, D. (2006a). Cultural, practical, and economic value of wild plants: A quantitative study in the Bolivian Amazon. Economic Botany 60:62-74.

Reyes-Garcia, V., Vadez, V., Tanner, S., Huanca, T., Leonard, W.R. and McDade, T. (2006b). Measuring what people know about the environment: A review of quantitative studies. Tsimane' Amazonian Panel Study Working Paper \# 21. Accessed on $6^{\text {th }}$ September 2010 via http://www.tsimane.org/working\%20papers/TAPS-WP-21TEKMETHODS-Jan-2006.pdf

Reyes-García, V., Vadez, V., Tanner, S., McDade, T., Huanca, T. and Leonard, W.R. (2006c). Evaluating indices of traditional ecological knowledge: A methodological contribution. Journal of Ethnobiology and Ethnomedicine 2:21-29.

Reyes-Garcia, V., Vadez, V., Huanca, T., Leonard, W.R. and McDade, T. (2007a). Economic Development and Local Ecological Knowledge: A Deadlock? Quantitative Research from a Native Amazonian Society. Human Ecology 35(3):371-377.

Reyes-Garcia, V., Marti, N., McDade, T., Tanner, S. and Vadez, V. (2007b). Concepts and methods in studies measuring individual ethnobotanical knowledge. Journal of Ethnobiolog 27(2):182-203.

Ribot, J.C. (2002) Democratic Decentralisation of Natural Resources: Institutionalising Popular Participation. Washington, DC, USA: World Resources Institute.

Ribot, J.C., Agrawal, A. and Larson, A.M. (2006) Recentralizing while decentralizing: how national governments reappropriate forest resources. World Development 34: 1864-1886.

Rights and Democracy. (2006). Indigenous Women and Militarization. In Information Kit: Portrait of Indigenous Women of Asia. The Asian Indigenous Women's Network (AIWN) and the Indigenous Peoples Alliance of the Archipelago (AMAN: Aliansi Masyarakat Adat Nusantara), in partnership with Rights \& Democracy. Available online at http://www.ddrd.ca/site/PDF/publications/indigenous/InfoKit

Robbins, P. (2012). Political Ecology: A critical introduction to geography. MA: John Wiley \& Sons.

Robson, C. (2002). Real world research: a resource for social scientists and practitionerresearchers. MA: John Wiley \& Sons.

Rocha Silva, A.J. and Cavalcante Andrade, L.H. (2006). Cultural Significance of Plants in Communities Located in the Coastal Forest Zone of the State of Pernambuco, Brazil. Human Ecology 34(3):447-465. 
Rocha, J.M. (2005). Measuring Traditional Agro-Ecological Knowledge: An Example from Peasants in the Peruvian Andes. Field Methods 17(4):356-372.

Rolling, N.G. and Wagemakers, M. A. E. (1998). Facilitating sustainable agriculture: participatory learning and adaptive management in times of environmental uncertainty. Cambridge: Cambridge University Press.

Ross, N. (2002a). Cognitive aspects of intergenerational change: Mental models, cultural change, and environmental behavior among the Lacandón Maya of southern Mexico. Human Organisation 61(2): 125-138.

Ross, N. (2002b). Lacandón Maya intergenerational change and the erosion of folk biological knowledge. In J.P. Stepp, F. S. Wyndham \& R. K. Zarger (Eds.), Biocultural Diversity. Proceedings of the Seventh International Congress of Ethnobiology (pp. 585-592). Athens, Georgia: University of Georgia Press.

Ross, N. and Medin, D. L. (2005). Ethnography and Experiments: Cultural Models and Expertise Effects Elicited with Experimental Research Techniques. Field Methods 17(2): 131-149.

Ruddle, K. (1993). The transmission of Traditional Ecological Knowledge. In J. Inglis (Ed.), Traditional Ecologicaol Knowledge: Concepts and Cases (pp. 17-31). Ottawa: International Program on Traditional Ecological Knowledge \& International Development Research Centre.

Ruddle, K., Hviding, E. and Johannes, R.E. (1992). Marine resources management in the context of customary tenure. Marine Resource Economics 7: 249-273.

\section{$\mathbf{S}$}

Salafsky, N. and Margoluis, R. (1999). Threat reduction assessment: a practical and costeffective approach to evaluating conservation and development projects. Conservation Biology 13(4): 830-841.

Salafsky, N., Margoluis, R., Redford, K.H. and Robinson, J.G. (2002). Improving the practice of conservation: a conceptual framework and research agenda for conservation science. Conservation Biology 16:1469-1479.

Salafsky, N., Salzer, D., Ervin, J., Boucher, T. and Ostlie. W. (2003). Conventions for defining, naming, measuring, combining, and mapping threats in conservation: an initial proposal for a standard system. Washington, DC: Conservation Measures Partnership.

Salafsky, N., Salzer, D., Stattersfield, A. J., Hilton-Taylor, C., Neugarten, R., Butchart, S. H. M., Collen, B., Cox, N., Master, L. L., O'Conner, S. and Willkie, D. (2008), A Standard Lexicon for Biodiversity Conservation: Unified Classifications of Threats and Actions. Conservation Biology 22: 897-911. 
Salafsky, N., Salzer, D., Stattersfield, A. J., Hilton-Taylor, C., Neugarten, R., Butchart, S. H. M., Collen, B., Cox, N., Master, L. L., O'Conner, S. and Willkie, D. (2009). Pragmatism and practices in classifying threats: Reply to Balmford et al. Conservation Biology 23(2): 488-93.

Sankhala, K. (1993). Return of the tigers. New Delhi: Lustre Press.

Sayad, A. (2004). The Suffering of the Immigrant. Cambridge, UK: Polity Press.

Schmink, M.,Redford, K.H. and Padoch. C, (1992). Traditional peoples and the biosphere: framing the issues and defining the terms. In K. H. Redford and C. Padoch (Eds.), Conservation of Neotropical Forests: Working from Traditional Resource Use ( $p p$. 3-13). New York: Columbia University Press.

Scholz, J. T. and Wang. C. (2006). Cooptation or transformation? Local policy networks and federal regulatory enforcement. American Journal of Political Science 50(1): 81-97.

Scheyvens, R. and Momsen, J.H. (2008). Tourism and poverty reduction: Issues for small island states. Tourism Geographies 10(1).

Schultes, R.E. (1992). Ethnobotany and Technology in the Northwest Amazon: A Partnership. In M. Plotkin and L. Famolare (Eds.), Sustainable Harvest and Marketing of Rain Forest Products (pp. 7-13). Washington, D.C.: Island Press.

Schultes, R.E. and Raffauf, R. F. (1990). The Healing Forest: Medicinal and Toxic Plants of the Northwest Amazonia. Portland, Ore.: Dioscorides Press.

Schwab, R.G. (1995). The calculus of reciprocity: principles and implications of Aboriginal sharing. CAEPR Discussion Paper No. 100, Centre for Aboriginal Economic Policy Research. Canberra: The Australian National University.

Schwartzman, S., Moreira, A. and Nepstad, D. C. (2000). Rethinking tropical forest conservation: perils in parks. Conservation Biology 14: 1351-1357.

Shadish, W.R., Cook, T.D. and Leviton, L.C. (1991). Foundations of program evaluation: theories of practice. Newbury Park, California: Sage Publication.

Shanley, P. and Rosa, N.A. (2004). Eroding knowledge: an ethnobotanical inventory in Eastern Amazonia's logging frontier. Economic Botany 58:135-160.

Shepherd, J.G. (1991). Special session on management under uncertainties. NAFO Scientific Council Study 16:189.

Sherman, K. and Laughlin, T. (1992). Large marine ecosystems monitoring workshop report. U. S. Department of Commerce, NOAA Tech. Mem. NMFS-F/NEC-93. 
Sillitoe, P. (2002). Globalising indigenous knowledge. In P. Sillitoe, A. Bicker \& J. Pottier (Eds.), Participating in Development: Approaches to Indigenous Knowledge (pp. 108-138). London \& New York: Routldge.

Sillitoe, P. (2007). Local science vs global science: An overview. In P. Sillitoe (Ed.), Local sciencevs. vs global science: Approaches to Indigenous Knowledge in international development. New York: Berghahn Books.

Sillitoe, P. (2010). Trust in development: Some implications of knowing in indigenous knowledge. Journal of the Royal Anthropological Institute 16(1): 12-30.

Silvano, R. A. M. and Begossi, A. (2002). Ethnoichthyology and fish conservation in the Piracicaba River (Brazil). Journal of Ethnobiology 22 (2): 285-306.

Silverman, D. (2005). Interpreting qualitative data: methods for analysing talk, text and interaction. London: SAGE Publication Ltd.

Silverman, D. (2009). Doing Qualitative Research. London: SAGE Publication Ltd.

Simpson, L., Wood, L. and Daws, L. (2003). Community capacity building: Starting with people not projects. Community Development Journal 38(4): 277-286.

Simpson, L. (2005). Traditional ecological knowledge among aboriginal people in Canada. In B.R. Taylor (ed.), Encyclopaedia of Religion and nature (pp.1649-1651). London: Thoemmes Continuum.

Smith, C., Heather, B. and Graeme, W.K. (2000). Globalisation and Indigenous Peoples: Threat or Empowerment? In G. Ward and C. Smith (Eds), Indigenous Cultures in an Interconnected World. St. Leonards, N.S.W.: Allen \& Unwin.

Smith, L. T. (1999). Decolonising Methodologies: Research and Indigenous Peoples. London: Zed Books Ltd.

Smith, L. T. (2005). On tricky ground: Researching the native in the age of uncertainty. In N. Denzin \& Y. Lincoln (Eds.), The SAGE Handbook of Qualitative Research (3ed). Thousand Oaks: SAGE.

Soemarwoto, R. (2007) Kasepuhan rice landrace diversity, risk management and agricultural modernisation. In R. Ellen (Ed.) Modern Crises and Traditional Strategies: Local ecological knowledge in Islands Southeast Asia (pp84-111). New York: Berghahn Books.

Solomon, M. (2004). Strengthening traditional knowledge systems and Customary Laws. In S.Twarog, and P. Kapoor (Eds.), Protecting and Promoting Traditional Knowledge: Systems, National Experiences and International Dimensions (pp.155166). Geneva: United Nations. 
Song, Y.Q. (2009). China case study - Participatory maize breeding and protection of farmers' rights. In IIED, Protecting community rights over traditional knowledge: Implications of customary laws and practices. London: IIED.

Song, L. (2010a). The study of Mongolian medicinal plants resources in Inner Mongolia [in Chinese]. China Ethnic Medicine Journal 16(1): 23-31.

Song, Y.Q. (2010b). Participatory plant breeding for strengthening traditional knowledge systems and farmers' rights in mixed communities in Guangxi, SW China. Paper presented at the 12th International Congress of Ethnobiology. Tofino.

Soselisa, H. L. (2007). A comparison of traditional and innovative subsistence strategies on Buano during periods of Socio-environmental stress, 1980-2003. In R. Ellen (Ed.), Modern Crises and Traditional Strategies: Local Ecological Knowledge in Islan Southeast Asia. (Vol. Studies in Environmental Anthropology and Ethnoecology). New York: Berghahn Books.

SPFII (Secretariat of the Permanent Forum on Indigenous Issues). (2005). Background note prepared by the Secretariat of the Permanent Forum for the International Workshop on Traditional Knowledge, Panama City, 21-23 September 2005. Doc. PFII/2005/WS.TK. Available online at http://www.un.org/esa/socdev/unpfii/ documents/workshop TK background note.pdf

Stavenhagen, R. (2005). Indigenous Peoples: An Essay on Land, Territory, Autonomy and Self-Determination. Available online at Land Research Action Network (LRAN) website http://www.landaction.org

Stavenhagen, R. (2006). Chapter II: Indigenous peoples: Land, territory, autonomy and self-determination. In P. Rosset, R. Patel, and M. Courville (Eds.), Promised Land: Competing Visions of Agrarian Reform. New York, USA: Institute for Food and Development Policy.

Stavenhagen, R. (2007a). Report of the Special Rapporteur on the Human Rights and Fundamental Freedom of Indigenous Peoples. UN document A/HRC/4/32 27 February 2007.

Stavenhagen, R. (2007b). Mission to Kenya. Addendum 3 to the Report Human Rights and Fundamental Freedom of Indigenous Peoples. UN document A/HRC/4/32 Add. 3 February 2007.

Steinfeld, H., Wassenaar, T. and Jutzi. S. (2006). Livestock production systems in developing countries: status, drivers, trends. Animal Production and Health Division, Food and Agriculture Organization of the United Nations. Rev. sci. tech. Off. int. Epiz. 25 (2): 505-516.

Stephenson, D. (2010). San reach landmark IPR benefit-sharing accord for diet pill. Cultural Survival. Accessed on $7^{\text {th }}$ December 2010 via http://www.culturalsurvival.org/publications/cultural-survivalquarterly/south-africa/san-reach-landmark-ipr-benefit-sharing-accord 
Stern, P. (2005). Deliberative methods for understanding environmental systems. BioScience 55: 976-982.

Sternberg, R.J., Nokes, C., Wenzel Geissler, P. , Prince, R. ,Okatcha, F., Bundy, D.A. and Grigorenko, E.L. (2001). The relationship between academic and practical intelligence: a case study in Kenya. Intelligence 29:401-418.

Strauss, A. L. and Corbin, J. M. (1997). Grounded theory in practice. London: SAGE publication LTD

Strauss, A. and Corbin, J. (1990). Basis of Qualitative Research: Grounded Theory Procedures and Techniques. USA: SAGE Publications.

Strauss, M. (1992). The World's Languages in Crisis. Language 68(1): 4-10.

Su, Y.Z., Li, Y.L., Cui, J.Y. and Zhao, W.Z. (2004). Influences of continuous grazing and livestock exclusion on soil properties in a degraded sandy grassland, Inner Mongolia, Northern China. Catena 59 (2005): 267-78.

Sundar, N. (2002). "Indigenise, nationalise, spiritualise" - an agenda for education? International Social Science Journal 54(173): 373-383.

Sutherland, W. J. (2003). Parallel extinction risk and global distribution of languages and species. Nature 423: 276-279.

Sutherland, W. J., Pullin, A. S., Dolman, P. M. and Knight. T. M. (2004). The need for evidence- based conservation. Trends in Ecology \& Evolution 19:305-308.

Swiderska, K. (2001). Stakeholder participation in policy on access to genetic resources, traditional knowledge and benefit-sharing. Case studies and recommendations. London: International Institute for Environment and Development.

Swiderska, K. (2004). Traditional Knowledge Protection and Recognition of Customary Law: Policy Issues and Challenges. Background paper prepared for the Planning Workshop on 'Protecting community rights over traditional knowledge: Implications of customary laws and practices', London 4-5 May 2004. IIED.

Swiderska, K. (2006). Banishing the biopirates: a new approach to protecting traditional knowledge. Gatekeeper Series - International Institute for Environment and Development, Environmental Economics Programme 2006(129): 24.

Swiderska, K. (2009). Protecting traditional knowledge: A framework based on customary-law and bio-cultural heritage. In K.N. Ninan (Ed), Conserving and valuing ecosystem services and biodiversity: economic, Institutional and social challeggens (pp338-49). London: Earthscan.

Swiderska, K., Argumedo, A., Pant, R., Vedavathy, S., Song, Y.Q., Mutta, D., Munyi, P., Herrera, H., Vedavathy, S. and Nellithanam, J. (2006). Protecting Community 
Rights over Traditional Knowledge: Implications of customary laws and practices: Interim Report 2005-2006. London: IIED.

Swiderska, K., Argumedo, A., Pant, R., Vedavathy, S., Song, Y.Q., Li, J.S., Mutta, D., Munyi, P. and Herrera, H. (2009a). Protecting Community Rights over Traditional Knowledge: Implications of customary laws and practices -Key findings and recommendations 2005-2009. London:IIED.

Swiderska, K., Roe, D., Siegele, L and Grieg-gran, M. (2009b). The governance of nature and the nature of governance: policy that works for biodiversity and livelihoods, London: IIED.

\section{$\mathbf{T}$}

Tang, R. (2007). Linking traditional ecological knowledge and sustainable resource management of saxoul in the Alashan region, Inner Mongolia of China. Master Thesis submitted to Victoria University of Wellington, New Zealand.

Tang, R. and Gavin, M. (2010). Traditional ecological knowledge informing resource management: Saxoul conservation in Inner Mongolia, China. Society \& Natural Resources 23(1): 193-206.

Tauli-Corpuz, V. (2008). Indigenous peoples' concept of development with culture and identity. Paper presented at the UNESCO/IASG/UNPFII round-table discussion on "Indigenous Peoples: Development with Culture and Identity" held in Paris 15 September, 2008.

Taylor, R. (2009). Community based natural resource management in Zimbabwe: the experience of CAMPFIRE. Biodiversity and Conservation 18(10):2563-2583.

Thaman, K. H. (2002). Towards cultural democracy in Pacific education: An imperative for the 21st century. In F. Pene, A. Taufe'ulungaki and C. Benson (Eds.), Tree of Opportunity: Re-thinking Pacific Education. Suva: Institute of Education, University of the South Pacific.

The National Bureau of Statistics China. (2008). 2008 National Statistical Report [in Chinese]. Accessed on $6^{\text {th }}$ May 2010 via http://www.stats.gov.cn/tjgb/nypcgb/qgnypcgb/t20080227 402464718.htm

The National Bureau of Statistics China. (2010). The Statistical Report of Economy and Development in Inner Mongolia, 2009 [in Chinese]. Accessed on $7^{\text {th }}$ May 2010 via http://www.stats.gov.cn/tjgb/ndtjgb/dfndtjgb/t20100302 402625748.htm.

Timothy, D.J. and Boyd, S.W. (2003). Heritage Tourism. Prenticel Hall: New Jersey, USA.

Timothey, D.J. (2011). Cultural Heritage and Tourism: An Introduction. Channel View Publications: UK. 
TNC (The Nature Conservancy). (2006). Conservation action planning: developing strategies, taking action, and measuring success at any scale. Arlington, Virginia: TNC. Available from http://conserveonline. org/workspaces/ cbdgateway/cap/resources/1/TNC_CAP_Basic_Practices.pdf/download

Tobe, K., Li, X., and Omasa, K. (2005). Effects of irrigation on seedling emergence and seedling survival of a desert shrub Haloxylon ammodendron (Chenmopodiaceae). Australia Journal of Botany 53: 529-534.

Tong, C., Wu, J., Yong, S., Yang, J., \& Yong, W. (2004). A landscape-scale assessment of steppe degradation in the Xilin River Basin, Inner Mongolia, China. Journal of Arid Environment. 59(1): 133-49.

Trask, M. (2009). Chapter VII: Emerging Issues. In DESA of UN, State of the World's Indigenous Peoples (pp. 219-238). United Nations Document. New York, USA.

Trevor, P. W. (1998). Indigenous knowledge and applied anthropology: Questions of definition and direction. Human Organization 57(3): 258-272.

Trotter, R.T. and M.H. Logan 1986 Informant Consensus: A New Approach for Identifying Potentially Effective Medicinal Plants. In N.L. Etkin (ed.), Plants in Indigenous Medicine \& Diet: Biobehavioral Approaches (pp. 91-112). New York: Redgrave Publishing Co.

Turner, T. (1990). Visual media, cultural politics, and anthropological practice: Some implications of recent uses of film and video among the Kayapo of Brazil. Commissions on Visual Aanthropology Review 1990 (spring): 8-13.

Twarog, S. And Kapoor, P. (Eds.). (2004). Protecting and Promoting Traditional Knowledge: Systems, National Experiences and International Dimensions. United Nations Conference on Trade and Development. Document No, United Nations, Geneva. UNCTAD/DITC/TED/10.

\section{$\mathbf{U}$}

Uma Shaanker, R., Ganeshaiah, K.N., Krishnan, S. Ramya, R.,, Meera, C., Aravind, N., Kumar, A., Rao, D., Vanaraj, G., Ramachandra, J., Gauthier, R., Ghazoul, J., Poole, N. and Chinnappa Reddy, B. (2004). Livelihood gains and ecological costs of non-timber forest products dependence: assessing the roles of dependence, ecological knowledge, and market structure in three contrasting human and ecological settings in south India. Environmental Conservation 31:1-13.

UN (United Nations).(1992). Agenda 21. Rio de Janeiro, Brazil: UN.

UN (United Nations). (2007). United Nations Declaration on the Rights of Indigenous Peoples. UN document. Accessed on 7th March 2011 via http://www.un.org/esa/socdev/unpfii/documents/DRIPS en.pdf 
UNCED (The United Nations Conference on Environment and Development). (1992) Capacity Building: Agenda 21's Definition, Chapter 37. New York: UNCED

UNDP (United Nations Development Programme). (1991) Symposium for Water Sector Capacity Building. Amsterdam: UNDP/International Institute for Hydraulic and Environmental Engineering.

UNDP (United Nations Development Programme). (1993). Human Development Report. Oxford: Oxford University Press.

UNEP (United Nations Environment Programme). (1992). The Rio Declaration. Rio de Janeiro, Brazil: UN.

UNEP (United Nations Environment Programme) and WTO (World Tourism Organisation). (2005). Making Tourism More Sustainable: A Guide for Policy Makers. Paris: UNEP \& WTO.

UNPFII (United Nations Permanent Forum on Indigenous Issues). (2005). Report on the Fifth Session (15-26 May 2006) UN Doc. E/2006/43.

UNPFII (United Nations Permanent Forum on Indigenous Issues). (2007). Report of the International Expert Group Meeting on Urban Indigenous Peoples and Migration, Santiago de Chile, 27-29 March 2007. UN Doc. E/C.19/2007/CRP.8.

UNPFII (United Nations Permanent Forum on Indigenous Issues). (2008). Report of the Secretariat on Indigenous Traditional Knowledge. UN Document.

UNU-IAS (United Nations University-Institute of Advanced Studies). (2010). Integration of Traditional Knowledge and Customary Law Concerns into ABS Policy. Accessed on 7 th March 2010 via http://www.ias.unu.edu/sub_page.aspx?catID=67\&ddlID=69

Usher, P. J. (1993). The Beverley-Kaminuriak Caribou Management Board: An experience in co-management. In J. Inglis (Ed.), Traditional Ecological Knowledge: Concepts and Cases (pp. 111-120). Ottawa: International Program on Traditional Ecological Knowledge \& International Development Research Centre.

\section{V}

Vanuatu Cultural Centre, (2005). Vanuatu Young People's Project. Retrieved on 13th October 2011 via http://www.vanuatuculture.org/site-bm2/ypp/vanuatuyoung-peoples-pro.shtml

Velez, M.A. (2011). Collective titling and the process of institution building: The new common property regime in the Colombia. Human Ecology 39: 117-29. 
Voeks, R. (2007). Are women reservoirs of traditional plant knowledge? Gender, ethnobotany and globalisation in northeastern Brazil. Singapore Journal of Tropical Geography 28(2007): 7-20

Voeks, R.A. and Nyawa, S. (2001). Healing flora of the Brunei Dusun. Borneo Research Bulletin 32:178-195.

Voeks, R. A. and Leony, A. (2004). Forgetting the forest: Assessing medicinal plant erosion in Eastern Brazil. Economic Botany 58: 294-306.

\section{W}

Walker, B. and Meyes, J.A. (2004). Thresholds in ecological and social-ecological systems: A developing database. Ecology and Society 9(2): article 3.

Walker, B., Holling, C.S., Carpenter, S. R. and Kinzi, A. (2004). Resilience, Adaptability and Transformability in Social-ecological Systems. Ecology and Society 9(2): 5. [online] URL: http://www.ecologyandsociety.org/vol9/iss2/art5

Walters, C. J. (1986). Adaptive Management of Renewable Resources. New York: McGrawHill.

Wang, R., Li, Z. Q., Cao, G. C., Chang, H. J., Wang, X. H., and Fu, L. (2002). Experiment on the use of Botulin type $\mathrm{C}$ against Rhombomys opmus in Haloxylon ammodendron stands [in Chinese]. Chinese Forestry 3: 45-49.

Wang, X. Y. (2007). Inefficient management under governmental policy [in Chinese]. Journal of Huazhong Normal University 2007 (5).

Wang, X. Y. (2009). Pastoral Communities under Environmental Pressure: Case Studies of Six Villages in Inner Mongolia. [in Chinese]. Beijing: Social Sciences Academy Press.

Wang, X. Y. (2010). Herder's Livelihood under the Shadow of Droughts [in Chinese]. Beijing: Social Sciences Academy Press.

Warren, D. M. (1991). Using indigenous knowledge in agricultural development. World Bank Discussion Papers. No. 127. Washington, DC: World Bank.

Warren, D. M. (1995). Comments on article by Arun Agrawal. Indigenous Knowledge and Development Monitor 4(1):13.

Warren, D. M., Slikkerveer, L.J. and Brokensha, D. (1995). The Cultural Dimension of Development: Indigenous Knowledge Systems. London: Intermadiate Technology Publications. 
Warren, D.M., Slikkerveer, L.J. and Brokensha, D. (eds.) (1996). The Cultural Dimension of Development: Indigenous Knowledge Systems. London: Intermediate Technology Publications.

Warren, D.M. and Pinkson, J. (1998). Indigenous African resource management of a tropical rain forest ecosystem: A case study of the Yoruba of Ara Nigeria. In F. Berkes, C. Folke \& J. Colding (Eds.), Linking Social and Ecological Systems: Management Practices and Social Mechanisms for Building Resilience (pp. 158181). Cambridge: Cambridge University Press.

Wavey, R. (1993). International Workshop on Indigenous Knowledge and Communitybased Resource Management In J. Inglis (Ed.), Traditional Ecological Knowledge: Concepts and Cases. (pp. 11-16). Ottawa: International Program on Traditional Ecological Knowledge \& International Development Research Centre.

Weber, R.J. and Larson, P. (2000). Indigenous Peoples and Conservation Organisations: Experiences in Collaboration. Washington, D.C.: World Wildlife Fund (USA).

Weiss, C.H. (1972). Utilisation of evaluation: toward comparative study. In C. H. Weiss (Ed.), Evaluating action programs: reading in social action and education (pp. 318326). Boston: Allyn and Bacon.

Wenger, E. (1999). Communities of Practices: Learning, Meaning, and Identity. Cambridge: Cambridge University Press.

Wester, L. and Yongvanit, S. (1995). Biological diversity and community lore in northeastern Thailand. Journal of Ethnobiology 15:71-87.

Williams, D.M. (1996). Grassland enclosures: Catalyst of land degradation in Inner Mongolia. Human Organisation 55(3): 307-13.

Williams, D. M. (2000). Representations of nature on the Mongolian steppe: An investigation of scientific knowledge construction. American Anthropologist 102(3): 503-519.

Williams, D. M. (2002). Beyond Great Walls - Environment, Identity and Development on the Chinese Grasslands of Inner Mongolia. Stanford: Stanford University Press.

Williams, N. M., and Baines, G. (Eds.) (1993). Traditional Ecological Knowledge: Wisdom for Sustainable Development. Canberra, Australia: Centre for Resource and Environmental Studies, Australian National University.

Wilmsen, C., Elmondorf, W. Fisher, L., Ross, J., Sarathy, B. and Wells, B. (2008) (Eds). Partnerships for Empowerment: Participatory Research for Community-based Natural Resource Management. London: Earthscan.

Wilson, D.C., Nielson, J.R. and Degnbol, P. (Eds.) (2003). The Fisheries Co-management Experience. Dordrecht, the Netherlands: Kluwer. 
WIPO (World Intellectual Property Organisation). (2011). The protection of Traditional Knowledge: Revised objectives and principles. Accessed on $11^{\text {th }}$ February 2012 via

http://www.wipo.int/edocs/mdocs/tk/en/wipo grtkf ic 18/wipo grtkf ic 185 . pdf

Wolfe, J., Bechard, C., Cizek, P. and Cole, D. (1991). Indigenous and Western Knowledge and Resource Management Systems. Canada: Guelph University Press.

Wolff, P. and Medin, D. L. (2001). Measuring the evolution and devolution of folkbiological knowledge. In L. Maffi (Ed.), On biocultural diversity. Linking language, knowledge, and the environment (pp. 213-227). Washington, D.C.: Smithsonian Institution Press.

Wondolleck, J. M., and Yaffee, S. L. (2000). Making Collaboration Work: Lessons from Innovation in Natural Resource Management. Washington, DC.: Island Press.

World Bank (2006b). China - Farmers Professional Associations Review and Policy Recommendations. Organisation Report Retrieved on 8th September 2009 via http://www.worldbank.org/research/2006/08/7077878/china-farmersprofessional-associations-review-policy-recommendations

WHO (World Health Organisation). (2008). Fact Sheet on Traditional Medicine. Retrieved on $26^{\text {th }}$ October 2010 via http://www.who.int/mediacentre/factsheets/fs134/en/

WGIP (Working Group on Indigenous Populations). (2006). Report of the Working Group on Indigenous Populations on its 24th Session, Geneva. UN Doc. A/HRC/Sub.1/58/22 14 August 2006.

Wu, J.J. and Boggess, W.G. (1999). The Optimal Allocation of Conservation Funds. Journal of Environmental Economics and Management 38(3): 302-321.

Wu, N.E. (2005). The Study of Ethnobotanical Knowledge of Ewenke Population in Hulun'beer [in Chinese]. Master thesis submitted to Inner Mongolia Normal University, China.

Wu, Z.L., Liu, H.M. and Liu, L.Y. (2001). Rubber cultivation and sustainable development in Xishuangbanna, China. International Journal of Sustainable Development and World Economy 8(4): 337-345.

Wuren, Q.M.G., Bao, X.X. and Yi, J. (2009). The effects of different herding methods on semi-desert vegetation on the Mongolian steppe [in Chinese and Mongolian]. The collection of the proceeding papers of the Forum of China and Mongolia Cooperative Projects 2009: Ulan Bator.

WWF (World Wildlife Fund) (2007). WWF Standards of Conservation Project and Programme Management. Can be accessed via www.panda.org/standards/complete 
Wynberg, R. and Swiderska, K. (2001). South Africa Experience on developing a policy on biodiversity and access to genetic resources. Participation in access and benefit-sharing. Case study No.1. IIED.

\section{$\mathbf{X}$}

Xiwu Qi Autonomous Region People's Congress. (2006). The Planning Outline of the $11^{\text {th }}$ Five-year (2006-2010) of Economy and Social Development in Inner Mongolia Autonomous Region [in Chinese]. Accessed on $7^{\text {th }}$ November 2011 via http://www.xwq.gov.cn/zwgk 21/fzgh/sywfzgh/201010/t20101022 524223.ht $\underline{\mathrm{ml}}$

Xiwu Qi Government. (2010). The Planning for 11th Five-year (2006-2010) regional economy in XiwuQi [in Chinese]. Accessed on 7th November, 2011 via http://www.xwq.gov.cn/zwgk 21/fzgh/sywfzgh/201010/t20101022 524225.ht $\underline{\mathrm{ml}}$

Xiwu Qi Statistics Bureau. (2011). The Annual Report of XiwuQi Economic and Social Development, 2010 [in Chinese]. Accessed on $5^{\text {th }}$ July 2012 via http://www.xwq.gov.cn/zwgk 21/tjsj

Xu, J.C. and Melick, D.R. (2007). Rethinking the effectiveness of public protected areas in southwestern China. Conservation Biology 21(2):318-28.

Xu, J.C., Yang, Y., Li, Z.Q., Tashi, N., Sharma, R. and Fang, J. (2008a). Understanding land use, livelihoods, and health transitions among Tibetan nomads: A case from Gangga township, Diqing County, Tibetan Autonomous Region of China. EcoHealth 5(2):104-114.

Xu, J.C., Sharma, R., Fang, J. and Xu, Y.F. (2008b). Critical linkages between land-use transition and human health in the Himalayan region. Environment International 34:239-247.

Xue, D.Y. (2009). On concepts and protection of traditional knowledge [in Chinese]. China Biodiversity 17(2):21-33.

\section{Y}

Ye, X. H. (2009). The study of Mongolian traditional ethnobotanical knowledge in Xilinguole Meng, Inner Mongolia [in Chinese]. Master Thesis submitted to Inner Mongolia Normal University.

Yeh, E.T. (2004) Property relations in Tibet since decollectivisation and the question of fuzziness. Conservation and Society 2:107-131. 
Yi, J., Ba, Y.X., and Liu, S.R. (2009). The study of the attributes of stipa grandis and the assessment system of grassland degradation on the Mongolian Steppe [in Chinese and Mongolian]. The collection of the proceeding papers of the Forum of China and Mongolia Cooperative Projects 2009: Ulan Bator.

Young, O. R. (2002a). The Institutional Dimensions of Environmental Change. Cambridge: MIT Press.

Young, O.R. (2002b). Institutional interplay. In E. Ostrom, T. Diaz, N, Dolsak, P.C. Stern, and E.U. Weber (Eds.), The Drama of Commons (pp.263-291). Washington, D.C.: National Academy Press.

Young, O. R. (2006). Vertical interplay among scale-dependent environmental and resource regimes. Ecological Society 11(1): 27-40.

Young, N., Zachernuk, T. and Fock, A. (2006). Amber light for farmers' organisations China Development Brief. 2006-10-15 17:02. Retrieved on 8th September 2009 via http://www.chinadevelopemntbrief.com/node/813

Young, O.R., King, L.A. and Schroeder, H. (2008) Institutions and Environmental Change. Cambridge: MIT Press.

$\mathbf{Z}$

Zarger, R. K. (2002). Acquisition and Transmission of Subsistence Knowledge by Q'eqchi' Maya in Belize. In J.R. Stepp, F.S. Wyndham and R.K. Zarger (eds.) Ethnobiology and Biocultural Diversity: Proceedings of the Seventh International Congress of Ethnobiology (pp. 593-603). International Society of Ethnobiology. Athens: University of Georgia Press.

Zarger, R. K. and Stepp, J. R. (2004). Persistence of Botanical Knowledge among Tzeltal Maya Children. Current Anthropology 45(3): 413-418.

Zent, S. (1999). The quandary of conserving ethnoecological knowledge: A Piaroa example.In T. L. Gragson \& B. G. Blount (Eds.), Ethnoecology: Knowledge, Resources, and Rights (pp. 90-124). Athens and London: The University of Georgia Press:

Zent, S. (2001). Acculturation and ethnobotanical Knowledge loss among the Piaroa Venezuela: Demonstration of a Quantitative Method for the Empirical Study of TEK change. In L. Maffi (Ed.), On Biocultural Diversity: Linking Language, Knowledge, and the Environment (pp. 190-211). Washington, DC: Smithson. Inst. Press.

Zent, S. and Maffi, L. (2009). Final Report on Indicator No. 2: Methodology for Developing a Vitality Index of Traditional Environmental Knowledge (VITEK) for the Project "Global Indicators of the Status and Trends of Linguistic Diversity and Traditional Knowledge." Terralingua. 
Zent, S. and Zent, E. L. (2004). Ethnobotanical convergence, divergence, and change among the Hoti of the Venezuelan Guyana. In J. S. Carlson \& L. Maffi (Eds.), Ethnobotany and the Conservation of Biocultural Diversity. New York: New York Botanical Garden Press.

Zent, S. and Zent, E.L. (2007). On Biocultural Diversity from a Venezuelan Perspective: tracing the interrelationships among biodiversity, culture change, and legal reforms. In C. McManis (Ed.) Biodiversity and the Law: Intellectual Property, Biotechnology \& Traditional Knowledge (pp.91-114). London: Earthscan/James \& James Publishers.

Zhang, X. (2005). The growth of Chinese economy [in Chinese]. Economics 3: 21-29.

Zhao, R.W. (2008). Reflection on the Economic Reform of China in the Past Thirty Years [in Chinese]. Contemporary Finance and Economics 9: 11-17.

Zhao, Z. H., Zhao, J., Wang, W. X., Tang, M. C., Li, P., and Pan, J. P. (2003). Control of Rhombomys opimus in Haloxylon ammodendron (Mey.) Bunge in western Inner Mongolia [in Chinese]. Flora Protection 4: 23-26.

Zimmerer, K.S. and Bassett, T.J. (Eds). (2003). Political Ecology: An Integrative Approach to Geography and Environment-Development Studies. New York: The Guilford Press. 


\section{Appendix 1.1}

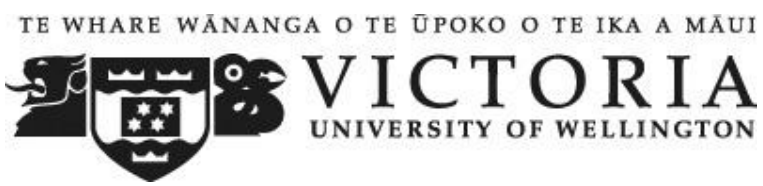

INFORMATION SHEET

(QUESTIONNAIRE)

Project: A Global Survey of Traditional Ecological Knowledge Conservation Options

Researcher: Ruifei Tang

PhD Candidate in Environmental Studies

Supervisors: Dr. Michael Gavin

Lecturer of Environmental Studies

Dr. Warwick Murray

Professor of Development Studies

\section{School of Geography, Environment and Earth Science}

Victoria University of Wellington, New Zealand

I am a PhD student in Environmental Studies at Victoria University of Wellington. As part of this degree I am undertaking a research project leading to a thesis. This project seeks to investigate global patterns of traditional ecological knowledge (TEK) conservation practice. It aims to explore TEK conservation options and to facilitate sharing of its 'best' practices across regions. The Victoria University Human Ethics Committee has granted ethical approval to this project. This is to ensure that you and other participants are protected from any possible harm arising from your participation in this project.

I am inviting TEK-focused researchers and organisations, and representatives of indigenous communities worldwide to participate in this study. I would like you to fill out the questionnaire which contains questions about TEK changes in your community or research location, causal factors of these changes, TEK conservation mechanisms and its assessment.

Should you feel the need to withdraw from the project, you may do so at any time before I analyse the data (before May 31 th 2011).

Responses collected will form the basis of my research project and will be put into a written report on an anonymous basis. It will not be possible for you to be identified personally. Only grouped responses will be presented in this report. All material collected will be kept confidential. No other person beside me and my supervisors, Dr. Michael Gavin and Dr. Warwick 
Murray, will see the questionnaires. The questionnaires will be destroyed five years after the end of the project.

A summary of the research results will be returned to you if you choose so (please see the option in the Questionnaire). I will also set up a web page to publish results in an aggregate form.

The thesis will be submitted for marking to the School of Geography, Environment and Earth Science and deposited in the Library of Victoria University of Wellington. The results of this research will be submitted for publication in scholarly journals, and /or for conference papers.

If you have any questions or would like to receive further information about the project, please contact me at:

School of Geography, Environment and Earth Science, Victoria University of Wellington P 0 Box 600, Wellington 6140, New Zealand

Email: trf615@gmail.com Phone: 0064-4-463 5233-8619

Or you can contact my primary supervisor, Dr Michael Gavin at the same address above.

Email: Michael.Gavin@vuw.ac.nz. Phone: 0064-4-463 5195

Researcher: Ruifei Tang

signed: Ruifei Tang Date: 


\section{Appendix 1.2}

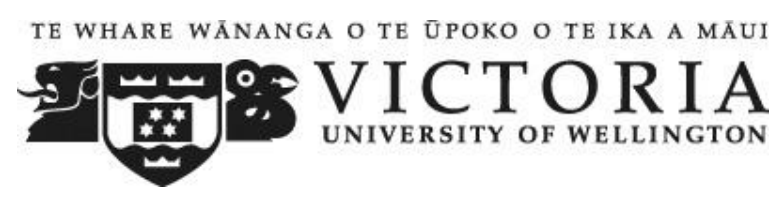

\section{INFORMATION SHEET}

(INTERVIEW)

Project: A Global Survey of Traditional Ecological Knowledge Conservation Options

Researcher: Ruifei Tang $\mathrm{PhD}$ Candidate in Environmental Studies

Supervisors: Dr. Michael Gavin Lecturer of Environmental Studies

Dr. Warwick Murray

Professor of Development Studies

\section{School of Geography, Environment and Earth Science} Victoria University of Wellington, New Zealand

Thank you for agreeing to be interviewed for providing more detailed information regarding my research. It is very important for the research to have your further participation and more detailed information. The University Human Ethics Committee has granted ethical approval to this project. This is to ensure that you and other participants are protected from any possible harm arising from your participation in this project.

I will interview you after having received your consent for participation. I will arrange an interview in an applicable form and meet your preference (face-to-face if we are in the same city or via phone call and/or internet communication). I will take notes during the interview, and transcribe them with your permission. It will not be possible for you to be identified personally. All material collected will be kept confidential. I will keep the interview notes and transcripts in a locked cabinet and on a computer with password access. Only my supervisors and I will have access to these materials. The interview notes and transcripts will be destroyed five years after the end of the project.

Should you feel the need to withdraw from the project, you may do so at any time before I analyse the data (before May 31 th 2011).

A summary of the research results will be returned to you if you choose so (please see the option in the Consent Form). I will also set up a web page to publish results in aggregate. 
The thesis will be submitted for marking to the School of Geography, Environment and Earth Science and deposited in the University Library. The results of this research will be submitted for publication in scholarly journals, and /or for conference papers.

If you have any questions or would like to receive further information about the project, please contact me at:

School of Geography, Environment and Earth Science, Victoria University of Wellington P 0 Box 600, Wellington 6140, New Zealand

Email: trf615@gmail.com Phone: 0064-4-463 5233-8619.

Or you can contact my primary supervisor, Dr Michael Gavin at the same address above. Email: Michael.Gavin@vuw.ac.nz Phone: 0064-4-463 5195.

Researcher: Ruifei Tang

Signed: Ruifei Tang Date: 


\section{Appendix 2}

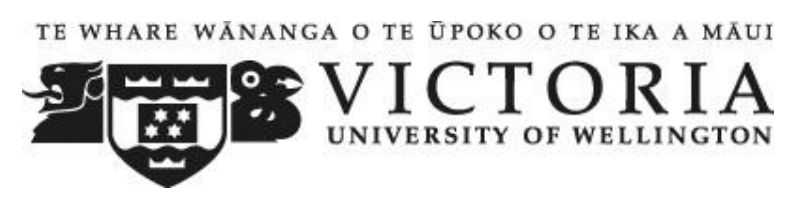

\section{CONSENT FORM}

Project: A Global Survey of Traditional Ecological Knowledge Conservation Options

Researcher: Ruifei Tang PhD Candidate in Environmental Studies

Supervisors: Dr. Michael Gavin Lecturer of Environmental Studies Dr. Warwick Murray Professor of Development Studies

\section{School of Geography, Environment and Earth Science}

Victoria University of Wellington, New Zealand

I have been given and have understood an explanation of this research project.

I have had an opportunity to ask questions and have them answered to my satisfaction.

I understand that I may withdraw myself (or any information I have provided) from this project (before the data to be analysed, by May 31th 2011).

I understand that the data I provide will not be used for any purpose other than that described to me in the Information Sheet.

I agree to the interview being transcribed.

I understand that I have the right to check the interview transcripts before publication.

I would like to receive a summary of the results of this research when it is completed.

Yes $\square$ No

If you ticked Yes, please provide your contact details below:

I agree to take part in this research.

Name of participant:

Signature:

Date: 


\title{
Appendix 3
}

TE WHARE WĀNANGA O TE ŪPOKO O TE IKA A MĀUI

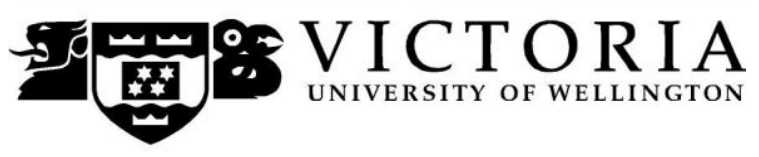

\section{MEMORANDUM}

\author{
Phone $\quad 0-4-4635676$ \\ Fax $\quad 0-4-4635209$ \\ Email_Allison.kirkman@vuw.ac.nz
}

\begin{tabular}{|c|c|}
\hline TO & Ruifei Tang \\
\hline COPY TO & Michael Gavin, Warwick Murray \\
\hline FROM & Dr Allison Kirkman, Convener, Human Ethics Committee \\
\hline DATE & March 29th 2010 \\
\hline PAGES & 1 \\
\hline SUBJECT & $\begin{array}{l}\text { Ethics Approval: No } 17360 \text { A global review of traditional } \\
\text { ecological knowledge change and it conservation options. }\end{array}$ \\
\hline
\end{tabular}

Thank you for your application for ethical approval, which has now been considered by the Standing Committee of the Human Ethics Committee.

Your application has been approved from the above date and this approval continues until 12 July 2012. If your data collection is not completed by this date you should apply to the Human Ethics Committee for an extension to this approval.

Best wishes with the research.

Allison Kirkman

Convener 


\section{Appendix 4}

\section{A Preliminary List of Potential Survey Participants - Organisations}

1. Bangladesh Resource Center for IK

2. Brazilian Resource Center for IK

3. Burkina Faso Resource Center for IK

4. Cameroon IK Organisation

5. CBD indigenous and local community

6. Center for Advanced Research on IK Systems, India

7. Center for Biodiversity and IK, Yunan, China

8. Center for Cosmovisions and IK, Ghana

9. Center for IK in Farm and Infrastructure Magt, Nigeria

10. Center for IK on Indian Bioresources, India

11. Center for IK on Population, Resources, and Environmental Magt, Nigeria

12. Center for IK, Sierra Leone

13. Center for international research and advisory networks (CIRAN/Nuffic)

14. Elliniko Resource Center for IK, Greece

15. Georgia Resource Center for IK

16. Ghana Resource Center for IK

17. Global Knowledge Partnership (GKP)

18. Indigenous Knowledge for Development Program of the World Bank's Africa

19. Indigenous Peoples' Restoration Network,

20. Indigenous Resource Study Center, Ethiopia

21. Indonesian Resource Center for IK

22. Interinstitutional Consortium for IK, USA

23. International Development Research Center

24. International Institute for Environment and Development

25. International Program on TEK

26. IUCN's inter-commission task force on Indigenous People

27. Kenya Resource Center for IK

28. L' rauravetl'an Information and Education Network of Indigenous People, Russia,

29. Maasai Resource Center for IK, Tanzania

30. Madagascar Resource Center for IK

31. Mexican research, Teaching, and Service Netork on IK

32. Nigerian Center for IK

33. Philippine Resource Center for Sustainable Development and IK

34. Russian Resource Center for IK

35. Society for Ecological Restoration International

36. South African Resource Center for IK

37. Sri Lanka Resource Center for IK 
38. TEK Working Group of IUCN

39. The resource Center for Aboriginal Forestry Issue in Canada "Forest Home"

40. UN Research Institute for Social Development (UNRISD)

41. UNESCO's Man and the Biosphere Program (MAB)

42. UNESCO's program in traditional magt sysyems in coastal marine areas

43. Uruguayan Resource Center for IK

44. Venezuelan Resource Secretariat for IK 
Appendix 5.1

TE WHARE WĀNANGA O TE ŨPOKO O TE IKA A MĀUI

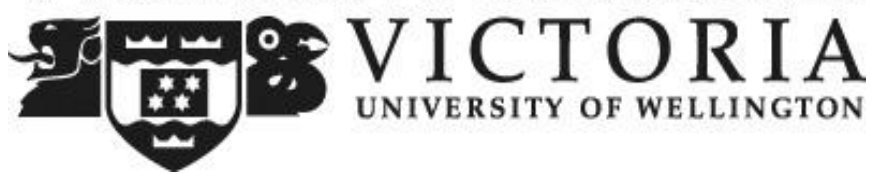

Traditional Ecological Knowledge

Conservation Options Questionnaire

(Researcher Version)

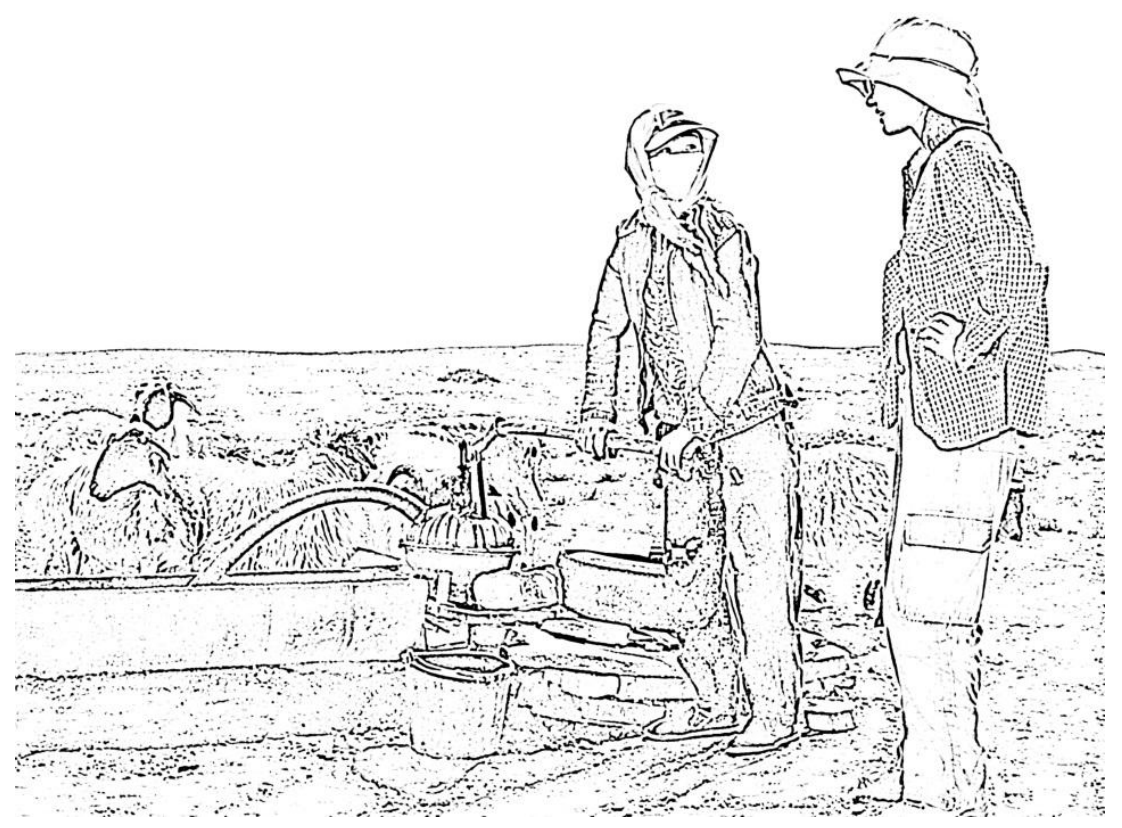

School of Geography, Environment and Earth Sciences Victoria University of Wellington

New Zealand 2010-2011

THANK YOU FOR YOUR PARTICIPATION! 
This research seeks to investigate global patterns of Traditional Ecological Knowledge (TEK) conservation practice. It aims to explore TEK conservation options and to facilitate sharing of its 'best' practices across regions.

For this questionnaire, Traditional Ecological Knowledge refers to the knowledge, practical skills, social institutions, worldviews and beliefs about the environment that traditionally belong to a community, and may still be in place.

We use TEK to represent Traditional Ecological Knowledge in this questionnaire.

\section{START HERE}

1. Where is your research location?

(Village / Community)

(State / Provincial region)

(Country)

2. How long have you worked in this location (duration)?

3. Which group/s of indigenous people do you work with (ethnicity)?

4. What is/are your research topic/s? 
5. Based on your experiences, how would you assess the general change of TEK in your research location over the last 50 years?

Please mark the appropriate box
$\square$ Dramatic loss
$\square$ Loss
$\square$ Slight loss
$\square$ No change (please skip to Question 7)
$\square$ Slight gain
$\square$ Gain
$\square$ Dramatic Gain
$\square$ Don't know
$\square$ Other (please specify below)

6. In your opinion, what are the main reasons for TEK change in your research location?

7. What has been done to conserve TEK in your research location? Which aspects of TEK have these conservation mechanisms targeted? (e.g. knowledge of plant and animal names; craft and tool making; traditional social organisation) 
8. How successful have these TEK conservation practices been in your research location? Why?

9. Which TEK conservation mechanisms are currently available in your research location? Please mark all appropriate boxes

Governmental policy support

Informal, community school for TEK

Including TEK into formal education

Co-management between government and indigenous community

Community-based biocultural conservation

Intellectual Property Rights system

Indigenous rights claims

Customary law or minority law

TEK workshops or conferences

Documentation and databases of TEK

Other (please specify below) 
Thank you for your time and the valuable details you provided!

Would you be willing to participate in a 10-15 minutes interview about TEK conservation?

$\square$ Yes $\quad \square$ No

If Yes, Please provide your contact details below

Name:

Email address:

Phone number and/or Skype name, and best contact time:

Would you like to receive a summary of the results of this research?
$\square$ Yes
$\square$ No

If Yes, Please provide your contact details below:

Name:

Email address or postal address:

If you have any questions about this study, please contact me

Ruifei Tang

School of Geography, Environment and Earth Sciences

Victoria University of Wellington

P.O. Box 600 Wellington 6140, New Zealand

trf615@gmail.com +64-211404448 
Appendix 5.2

TE WHARE WĀNANGA O TE ŨPOKO O TE IKA A MĀUI

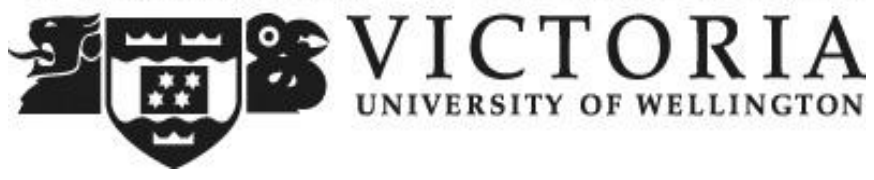

Traditional Ecological Knowledge

Change and Its Conservation Options

Questionnaire

(Community Version)

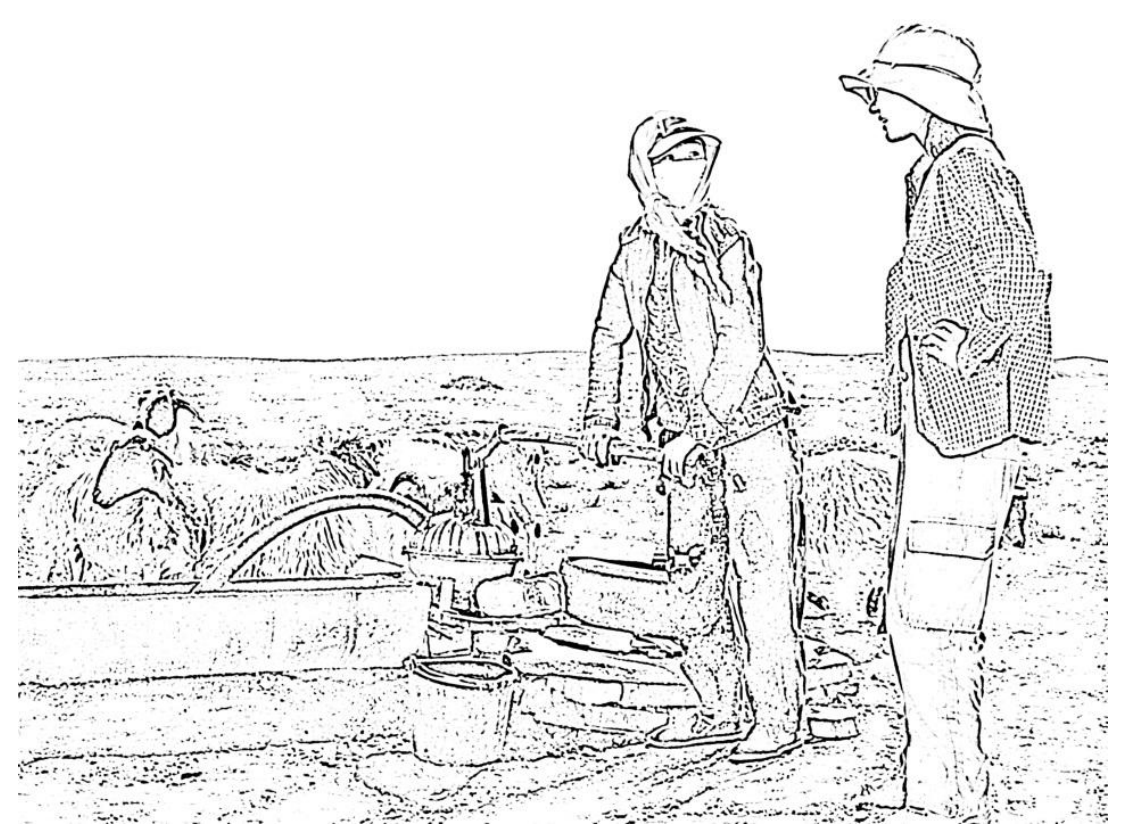

School of Geography, Environment and Earth Sciences Victoria University of Wellington

New Zealand 2010

THANK YOU FOR YOUR PARTICIPATION! 
This research seeks to investigate global patterns of Traditional Ecological Knowledge (TEK) conservation practice. It aims to explore TEK conservation options and to facilitate sharing of its 'best' practices across regions.

For this questionnaire, Traditional Ecological Knowledge refers to the knowledge of the environment you live in (including the land, plants, animals, natural resources and climate), production practices, social organisation, rules and beliefs that you and your community traditionally have had or used to have.

We use TEK to represent Traditional Ecological Knowledge in this questionnaire.

\section{START HERE}

\section{Where do you live?}

(Village / Community)

(State / Provincial region)

(Country)

2. How long have you lived in this location?

3. What is your ethnicity? Please specify if you belong to a particular tribe or group

4. Has there been any study of TEK in your location?

Yes

No

Don't know

If Yes, by whom? 
5. Based on your experiences, how would you assess the general change of TEK in your location over the last 50 years?

Please mark the appropriate box
$\square$ Dramatic loss
$\square$ Loss
$\square$ Slight loss
$\square$ No change (please skip to Questions 7)
$\square$ Slight gain
$\square$ Gain
$\square$ Dramatic Gain
$\square$ Don't know
$\square$ Other (please specify below)

6. In your opinion, what are the main reasons for TEK changes in your location?

7. What has been done to conserve TEK in your location? (This can be initiated from your own community, or initiated by an outside party) 
8. How successful have these TEK conservation practices been in your location? Why?

9. Which TEK conservation mechanisms are currently available in your location? Please mark all the appropriate boxes

Governmental policy support

Informal, community school for TEK

Including TEK into formal education

Co-management between government and indigenous community

Community-based biocultural conservation

Intellectual Property Rights system

Indigenous rights claims

Customary law or minority law

TEK workshops or conferences

Documentation and databases of TEK

Other (please specify below) 
Thank you for your time and the valuable details you provided!

\author{
Would you be willing to participate in a 10-15 minutes interview about \\ TEK conservation? \\ $\square$ Yes $\square$ No
}

If Yes, Please provide your contact details below

Name:

Email address:

Phone number and/or Skype name, and best contact time:

Would you like to receive a summary of the results of this research?
$\square$ Yes
$\square$ No

If Yes, Please provide your contact details below:

Name:

Email address or postal address:

If you have any questions about this study, please contact me Ruifei Tang

School of Geography, Environment and Earth Sciences

Victoria University of Wellington

P.O. Box 600 Wellington 6140, New Zealand

trf615@gmail.com 0064-4-463 5233-8619 
Appendix 5.3

TE WHARE WĀNANGA O TE ŨPOKO O TE IKA A MĀUI

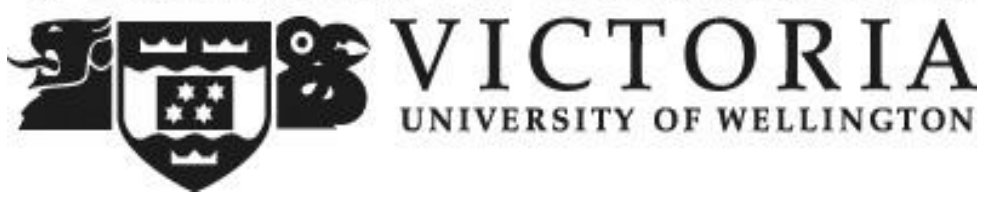

Traditional Ecological Knowledge

Change and Its Conservation Options

Questionnaire

(Organisation Version)

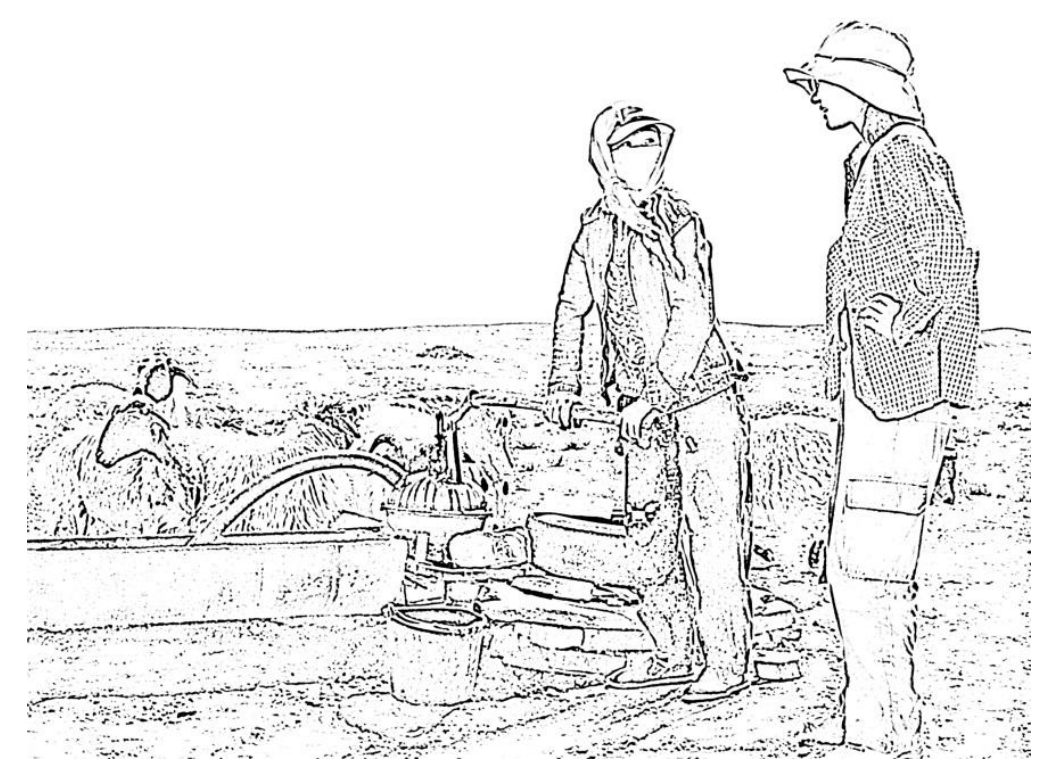

School of Geography, Environment and Earth Sciences Victoria University of Wellington New Zealand 2010

THANK YOU FOR YOUR PARTICIPATION! 
This research seeks to investigate global patterns of Traditional Ecological Knowledge (TEK) conservation practice. It aims to explore TEK conservation options and to facilitate sharing of its 'best' practices across regions.

For this questionnaire, Traditional Ecological Knowledge refers to the knowledge, practical skills, social institutions, worldviews and beliefs about the environment that traditionally belong to a community, and may still be in place.

We use TEK to represent Traditional Ecological Knowledge in this questionnaire.

\section{START HERE}

1. Which area does your organisation focus on?

(Village / Community)

(State / Provincial region)

(Country)

2. How long has your organisation worked in this location (duration)?

3. Which group/s of indigenous people do you work with (ethnicity)?

4. What is/are your main project/s? 
5. Based on your experiences, how would you assess the general change of TEK in this location over the last $\mathbf{5 0}$ years? Please mark the appropriate box
$\square$ Dramatic loss
$\square$ Loss
$\square$ Slight loss
$\square$ No change (please skip to Question 7)
$\square$ Slight gain
$\square$ Gain
$\square$ Dramatic Gain
$\square$ Don't know
$\square$ Other (please specify below)

6. In your opinion, what are the main reasons for TEK change in this location?

7. What has been done to conserve TEK in this location? Which aspects of TEK have these conservation mechanisms targeted? (e.g. knowledge of plant and animal names; craft and tool making; traditional social organisation) 
8. How successful have these TEK conservation practices been in this location? Why?

9. Which TEK conservation mechanisms are currently available in this location?

Please mark all appropriate boxes

Governmental policy support

Informal, community school for TEK

Including TEK into formal education

Co-management between government and indigenous community

Community-based biocultural conservation

Intellectual Property Rights system

Indigenous rights claims

Customary law or minority law

TEK workshops or conferences

Documentation and databases of TEK

Other (please specify below) 
Thank you for your time and the valuable details you provided!

Would you be willing to participate in a 10-15 minutes interview about TEK conservation?
$\square$ Yes
$\square$ No

If Yes, Please provide your contact details below

Name:

Email address:

Phone number and/or Skype name, and best contact time:

Would you like to receive a summary of the results of this research?

$$
\square \text { Yes } \quad \square \text { No }
$$

If Yes, Please provide your contact details below:

Name:

Email address or postal address:

If you have any questions about this study, please contact me

Ruifei Tang

School of Geography, Environment and Earth Sciences

Victoria University of Wellington

P.0. Box 600 Wellington 6140, New Zealand

trf615@gmail.com +64-211404448 


\section{Appendix 6}

\section{A list of Indigenous Groups Covered by the Survey}

Location of the respondent

(Ordered by regions)

\section{AFRICA}

Papatia, Atakora Region, Benin

Korup area, Cameroon

Province Orientale, Congo

Paphos district, Cyprus.

Kenya, Tanzania, Uganda

Mpala Farm, Laikipia District, Kenya

Tsavo Conservation Area, Kenya

Lake Victoria Wetland Communities in

\section{Kenya}

Mbirikani, Kajiado, Kenya

Western province, Luhya community,

\section{Kenya}

Wamba Samburu, Kenya

Samburu District Kenya

Mavrovo National Park, Polog and

southwestern regions, Macedonia

Laosbolivia,Vietnam,Cambodia, Morocco

Matutuine District, Maputo Province,

\section{Mozambique}

Kalakari Desert, South Africa

Hluhluwe, KwaZulu-Natal, South Africa

South Nguru Mountain Block, Morogoro

Region, Tanzania

Bagamoyo District \& Kilimanjaro District,

\section{Tanzania}

Musoma- Mara, Tanzania

Arusha, Tanzania

Esiralei, Monduli, Arusha -Tanzania

Kampala City, Uganda

Kibale National Park, Kabarole District,

Uganda
Indigenous groups
Fulani

Edjagham

Mambasa,

Greek-Cypriot

Luo, Kikiyu, Maasai, Samburu, Luhya, Rendille,

Pokhot, Meru, Kisii, Kamba, Kalenjin

Maasai, Samburu, Turkana, Mukogodo

Rural Kenyans - Taita, Wakamba, Maasai, also in

Northern Kenya with Samburu and some Meru

Mostly Luo Tribe

Maasai

Luhya

Maasai-Samburu indigenous tribe

Samburu

Slavic speaking Macedonians / Albanian speaking Macedonians

Brou, Kri, Saek. Bidayo

Ronga \& Zulu

San

Zulu

Wanguu

Chagga

Kurya people of nrth west Tanzania

Arusha, Maasai, Chagga, Gogo and Bena

Maasai (pastoralists)

Basoga

Batooro tribe

\section{ASIA}

\section{Altai Republic}

Tangail, Mymensingh, Kushtia Dhaka, 


\section{Bangladesh}

Yunnan, China

Shanxi, China

Inner Mongolia, China

Qinghai-Tibetan plateau, China

Xidang, Yunnan, China; Luozong, Yunnan,

\section{China}

Anamalai Hills(Valparai taluk of

Coimbatore District in Tamil Nadu State \& adjoining Idukki district of Kerala State,

India

Place: PURI, State: Orissa, India

pilvai, Gujarat, India

Aurangabd- Maharashtra state, India

Bomdila, Arunachal Pradesh, India

Maharashtra, India and Jammu \& Kashmir,

\section{India}

Jenkapur, West Bengal, India

Villages of Sathyamangalam Forest

Division, Eastern Ghats of southern India Jannaram (Kawal Wildlife Sanctuary),

Andhra Pradesh, India

Meghalaya, India

Koraput, Orissa, India

District Sonebhadra, Uttar Pradesh, India

District Sonebhadra, Uttar Pradesh, India

Sabangau, Central Kalimantan, Indonesia

Pantar, NTT, Indonesia

\section{Mongolia}

Chitwan, Nepal

Chitwan National Park, Nepal

Chitwan district of Nepal.

\section{All 74 districts of Nepal}

F.R.Bannu (Fata), Pakistan

Northern Areas of Pakistan, Khunjerab

national Park, Pakistan

\section{Philippines}

Thung Yai Naresuan Wildlife Sanctuary,

Kanachanaburi, Thailand

20 locations in Thailand

Northwest Vietnam
Yao, Lisu, Zhuang, Dai, Naxi

Han Chinese

Mongolian

Tibetan

\section{Kham Tibetan}

Muduvan tribe in the Anamalai hills in India / Toda, Mullu Kurumba and Alu Kurumba tribes in Nilgiris of Tamil Nadu in India

Hindu Temple Priests

tribals of Gujarat like Bhil, Grasia, Dongari

Triable like Bhil, Thakur etc.

Khowas people

Dhangar, Thakkar, Maratha, Mali, Changpa

Mixed caste group community

Mostly tribal community

Gond tribe, Villagers

Khasi

Paroja, Kondhs, Godaba

The dominating tribal/ethnic groups of the area are Gond, Kharwar, Oraon, Kol, Baiga etc

Hindi

Dayak, Javanese, Banjarese (mostly)

Alor

Khalkha Mongol herders

Tharu

Tharu (indigenous people of lowland Nepal)

I am from Brahamin.

Especially, i do work with the disadvantage group of society.

WAZIR

Wakhi Speaking People

Aytas

Karen

Bru, Chong, Khmer, Kuy, Lao, Mon, Nyahkur (Chaobon), Phu Thai, Phuan, Saek, So, Tai Dam, Viet, Kaloung, Yoy, Yaou, Mong

kinh, hmong, thai 


\section{NORTH AMERICA}

Mashteuiatsh, Quebec, Canada

1. Pikangikum First Nation, Ontario,

\section{Canada}

Winnipeg, Manitoba, Canada

Yellow Head Nation, Upper Fraser River

Community Tete Juan , British Columbia,

\section{Canada}

Sliammon, BC, Canada

Desolation Sound, British Columbia,

\section{Canada}

Nunavut, Northwest Territories, Northern

Manitoba, Canada

WUikinuxv, Nuxalk, Kitasoo, Heiltsuk

communities in the central coast of British

Columbia, Canada

Klemtu, BC , Canada

southwest Yukon Territory and Nunavut

Territory, Canada

Texas, USA

Sahaptin, Columbia Plateau, Pacific

Northwest, USA

Alaska, USA: Interior Koyukon Athabascan

villages

Palolo, Hawaii, USA

Hayden, Colorado, USA

Indiana and Oklahoma, USA

Missouri, USA

Maryland, USA

Lawrence, Kansas, USA

Cibecue, Arizona, USA

Cibecue (Dishchii bikoh), Arizona, USA

Guam, Mariana Islands, USA

Littleton, Colorado, USA

Cherokee, North Carolina, USA

wisconsin, USA

Davis California, USA
Innu (Montagnais)

Anishinaabe (Ojibwa)

First Nations in Canada, right now its Cree, Micmac and Coast Salish communities

Cree, Cariboo, Anishinabe, Shuswap

Sliammon

Tla'amin (Sliammon) (Northern Coast Salish)

Dene, Metis, Inuit, Cree, Stoney

Kitasoo, Heilstuk, Nuxalk and WUikinuxv First

Nations

Kitasoo Band-First nation

Southern Tutchone, Inuit

Local hispanics

Sahapin (Yakama, Umatilla)

Koyukon Athabascans

Hawaiian

Euro-American ranchers

myaamia (formerly called, Miami)

N/A / I work with modern Missouri residents and past and present fauna

European Americans

Great Plains Indians

Western Apache

White Mountain Apache, American Indian

Indigenous Pacific Islander - Chamorro

Hispanic

The Eastern Band of the Cherokee Indians

Native Americans

portuguese

\section{SOUTH AMERICA}

Cieneguillas, Jujuy, Argentina / Suripujio, Jujuy, Argentina / Lagunillas del Farallón, Jujuy, Argentina / Susques, Jujuy, Argentina several indigenous communities along Negro river and Purus River, at Amazonas

Kollas - Aymaras - Atacameños

Bare and Jamamadi 
state, Brazil

Central Costa Rica.

Pira Parana basin in the Colombian

Amazon, Colombia

Sierra Nevada de Santa Marta, Colombia

Ciudad de Loja, Canton Loja, Provincia de

Loja, Ecuador

Pino Gordo [Choreachi] Chihuahua, Mexico

\section{Campeche, Mexico}

Mexico, various (through my students)

1) Tenejapa, Chiapas, Mexico; 2) San Juan

Mixtepec, Oaxaca, Mexico

Yucatan, Mexico;

Thirteen villages in two provinces (Panama and Darien provinces) in Panama

Huamachuco and surrounding villages, La

Libertad, Peru

Loreto, Peru - Peruvian Amazon

Infierno \& Sonene, Peru

Piaroa territory, Amazonas State,

\section{Venezuela}

\section{EUROPE}

Belgium, Brussels, Evere

Mâcon, Saône-et-Loire, France

Basilicata region of southern Italy

Norway in addition to southeastern Europe - the western Balkans

Grandola, Portugal

Serra da Abobereira, Porto-Marco de

Canauesses, Baiao, Amarhute, Portugal

Sesimbra, Portugal

\section{Russia}

University of Aberdeen, Scotland

Jaca, Spain

Geneva, Switzerland

Hereford, Hereford Shore, UK

\section{Costa Ricans}

Cubeo and Tikuna families (7 different groups)

Kogis, Arzarios, Arhuacos and Kankuamos

Saraguros

Raramuri (Tarahumara)

Ch'ol

nahua, popoloca, mazateco

1) Tzeltal Mayan, 2) Sierra Sur Zapotec, Huna Tlingit Local hispanics, Mexicans and Costa Ricans.

Embera, Kuna, and descendants of African slaves who have lived in the region for 500 years

Spanish-speaking Peruvians /

Maijuna (Orejon)

Ese Eja, They Live in Madre de Dios, Perú and Pando, Bolivia.

Piaroa, Yabarana

\section{Belgian}

French

autochthonous Italians and Arbereshe (ethnic

Albanian) communities

We really do not formally recognise indigenous people in our work - regarding all Europeans as being indigenous. The only exception concerns the Sami (Lapps) of northern Norway - which have a formal indigenous status - although this is based on their minority status rather than on any formal claim to prior occupation of land.

portuguese

Local intabitants

It's a fishermen community

Russian

Spaniards (Aragonese)herders and community Wichi, Aramkbut, Mapuche, Miskito, Tsimihety Welsh/English 
OCEANIA

Gordonvale, Cairns, state of Queensland, Australia

Indigenous Australian, Malanbarra Yidinji clan

Gamaroi and Yularoi country, northern

New South Wales, Australia (6 hours

north-west of Sydney).

Marshall Islands

Majuro, Marshall Islands

Several Ngāi Tahu communities that are promulgating or managing mātaitai and taiāpure for customary fishing and ecological restoration of kai species, mainly in coastal and estuarine habitats, but increasingly in rivers (and soon lakes). / Ngai Tahu's takiwā / Aotearoa / New

\section{Zealand}

\section{NZ and Pacific}

Dunedin, NZ

Ngati Raukawa ki te tonga, Te Ati Awa ki Whakarongotai and Ngati Toarangatira tribal area, Lower North Island, New

\section{Zealand}

Kahua, Makira ULawa Province, Solomon Islands

Wellington, New Zealand

Oneroa (village), Waiheke Island, Hauraki Gulf / Auckland, New Zealand. /

(Emphasis for this survey is on the Hauraki Gulf.)

Malekula Island, Vanuatu (Tisvel, Unmet, Mahavo and Dixon Reef as primary research communities)
Aboriginal Australian (Gamaroi and Yularoi). Marshallese (Micronesian)

Marshall Islanders /

Ngāi Tahu, Maori

all

Maori

Ngati Raukawa ki te tonga, Te Ati Awa ki Whakarongotai, Ngati Toarangatira Indigenous Solomon Islanders (Melanesians) who come from Kahu and speak Kahua (total number of people speaking this language is around 20,000)

Maori

Maori

Ni-Vanuatu; four language groups (Novol at Dixon Reef, Avava at Tisvel, Ninde at Mahavo, Ven Taut at Unmet) 


\section{Appendix 7}

\section{A list of the Research Subjects Covered by the Survey}

The project is called Te Tiaki Mahinga Kai see www.mahingakai.org.nz Specific aims and processes are reported in the research projects section of the website, along with the overall mission on the home page.

The protection of traditional ecological knowledge, intellectual property rights of indigenous people, and cultural heritage rights

integration of TEK and scientific knowledge in freshwater management

Ethnoecology / livelihood of forest and mountain tribes

Related to use of wildgrowing resources, mainly

TEK transmission of palms in 2 Ese Eja communities

ROLE OC CLIMATIC FACTORS ON GROWTH AND PHYSIOLOGY OF OIL YIELDING CROPS

bushmeat and livelihood issues related to conservation of wildlife

traditional knowledge about repellents against disease transmitting insects, with a special focus on malaria

Ecological change and restoration, in terms of treatments, effects of treatments, values, biocultural resources, indicators and mechanisms of ecological change.

Sustainable traditional methods of running the day to day activity of the temple of Lord Jagannath at Puri

Sharing knowledge of medicinal plants

LOCAL DEVELOPMENT IN RURAL ANDEAN COMMUNITIES. ANALISIS OF THE

ALTOANDINAS AND PUNEÑAS COMMUNITIES ATTITUDES AND TRADITIONAL

KNOWLEDGE TOWARD THE WILD VICUÑA (VICUGNA VICUGNA)

IK of ecological disturbance and renewal / Traditional use of fire

Traditional plants and animal guidebook development, institutional adaptative capacity of

Aboriginal communities in regards to climate change, integrated water resource

management planning

medical ethnobotany, ethnopharmacology, folk medicine

gathering extant ethnobotanical knowledge. reintroducing traditional harvesting and management regimes to study autecological affects on plants

Ethics, archaeology, cultural heritage

Traditional knowledge for Forest Management

ethnobotanical studies of north gujarat

Forst wealth and utility of plants in Aurangabad Distric M.S.

extirpation of species in the region / future management/conservation planning (incorporating local knowledge and perceptions about the species being managed)

1) Exploring differing epistemological perspectives on TEK via collaborative research methodologies / 2) Exploring how TEK is changing with changing demography, economy and social structure.

Heritage stewardship policy and practice

Biocultural diversity, biodiversity conservation in traditional agricultural systems, biodiversity change and implications for human adaptation, global environmental change, biofuels, meat, global markets and land use change

Change/loss and conservation of TEK on Malekula 
TEK about famine food

Issues concern conflicts between large carnivore conservation and local interests - such as reindeer / livestock herding, game hunting and other rural activities.

Health Care, Agriculture, Household Risk, HIV/AIDS, TB

Local knowledge and perceptions about ecology and behaviour of large carnivores

Factors that shape attitudes towards wildlife and change in attitudes

Wildlife poilcy

Forest based ecosystem services and biodiversity conservation

Climate Change Adaptation

Interactions of livestock and biodiversity; restoration

tradition of sustainable agriculture and fishery

Population Dynamics of Marco Polo Sheep

Community participation in conservation

Presence-absence surveys for wild felids outside tiger reserves in India

Ecological restoration following abandoned subsistence agriculture

ethnobotany esp. by childbirth / ethnobotany as a tool for village development

Social, economic and environmental assessment of voluntary resettlement in Padampur,

Chitwan National Park, Nepal

wildlife health, landscape change, dog health

ethnoecology, ICDPs, public participation in conservation

Maori participation in decision making

Collection of taditionl knowledge to inform marine use planning and marine spatial

planning

Building a marine use plan / Collecting traditional and current fishing areas / Traditional stories / Place names-in two traditional languages / Trade and how is has change over time / Marine resources / / So far we have completed three studies, TEK Study, Community needs study, and informal trade

the relationships between science and TEK in wildlife conservation, decision-making processes and governance for wildlife and ecosystems (more generally)

Ethnobotany; Plant conservation

Contributions of Victoria Farming and Fishing Project in Poverty Reduction in Musoma, Mara Region.

How development aid has influenced policy implementation

Ethnobiology, ethnoecology, community-based conservation

ethnobiological classification and nomenclature, ethnoecology of subsistence, Indigenous rights

$1 \mathrm{PhD}$ research and thesis titled "Cataloguing Culture: In Search of the Origins of Written Records, Material Culture and Oral Histories of the Gamaroi, Northern New South Wales": traditional custodianship and knowledge (linguistic, social, ecological/environmental, spiritual) - connection with the land and the relationship between these; / 2 Ongoing small research projects on a variety of topics - currently writing a conference paper titled "From a Woman's Breast to the Southern Sky: Body Landscape in Aboriginal Culture", inspired by Gamaroi land and sky features

predator wildlife human conflict

traditional plant knowledge, classification and use; ethno-ecology

conservation and management and belief issues relating to sacred groves 
Orang-utan and gibbon behavioural and feeding ecology; forest phenology; biodiversity surveys and monitoring

Assessment of environmental contaminants in neotropical migratory birds using the yellow rumped warbler as an indicator species.

Local knowledge of wildlife and habitats

Management and distribution of agricultural biodiversity, changes in land-use and agricultural systems

Medicinal plants

LEK and it's influence on natural resoures use and management by indigenous people.

Biodiversity conservation - integration of western/ international concepts and local approaches and building adaptive local institutions.

Values of the living environment / Risk analysis / Attitudes to genetic engineering / Organic growing practices and principles

Ethnobotanics

Sacred groves

1. Traditional agricultural practices in rural mountain landscapes / 2. Ecosystem services /

3. Human well-beings / 4. Biodiversity / 5. Erosion of TEK

The ethno-Ornithology of "edible birds" in Kenya; the significance of Quails (tsisindy) as a source of income by Luhya community and status of the affected species

Plant conservation, botanical gardens, high altitude medicinal plants

marine conservation and livelihoods

adaptation, human-environment interaction, climate change, resource management, landscape ecology, historical ecology

impacts of new protected area models on local livelihoods; resident perceptions of conservation and tourism authorities; diffusion of conservation and tourism institutions in China

Conservation and resettlement

Human-wildlife conflict mitigation and tolerance with specific reference to lions

geography and politics of natural resource management

Biodiversity, Environmental Science, Botany

Protected areas and biodiversity conservation governance

Resilience / Vulnerability / Human-environment relationships / Social and environmental change

How have orchard systems developed and changed through time as management units? / How is cider produced and embedded with cultural meaning as part of local cultural through time?

Archaeological - intertidal resource use through time, looking at intertidal resource features and the remains of resources (bone and shell remains)

Distribution of Medicinal plants especially used by tribes of Sonebhadra (U.P.)under normal and degraded environmental conditions

traditional ecological knowledge and local knowledge / intergration of science and local

knowledge/TEK / community-based natural resource management/collaborative management / rangeland ecology and management / resilience of social-ecological systems

Origins, distribution and utilisation of a crop plant (taro) that has spread between different geographical and cultural regions over thousands of years as a widely shared plant 
resource.

Freshwater management

T/LEK of traditional morel mushroom harvesting

The main topic is ifthe legal recognition of human collective rights has had any impacts in biodiversity conservation and management from a bottom-up basis

Ethno medicinal plant survey

Why Hawaiian language immersion is the most appropriate or best schooling for my daughters

medicinal plants, dye plants, fungi

agriculture

integration of scientific and traditional ecological knowledge of assessing semi- arid rangeland

Ethnomedicinal plants used by the aboriginals of Koraput, Orissa. 


\section{Appendix 8.1}

\section{INTERVIEW QUESTIONS}

-- Mongolian Community Members

The interview began with a few general questions:

1. How much do you remember about the traditional nomadic herding and traditional community organisation?

2. When did you stop using these traditional practices?

3. Why did their use end?

These questions tend to be very broad. The purpose of this broad beginning is to create a space for interviewees to decide how much details they want to recall and tell. Then the questions will focus on the recovery of traditional nomadic herding practices and the revival of traditional institutions under the form of new cooperative practices. Questions included:

4. Do you want the traditional nomadic herding and traditional institutions in use again?

5. If so, what are the main threats to the implementations? If not, why?

6. Will this new law address these threats?

7. How do you and your community carry out cooperative production now?

8. How have your lives and income levels changed?

9. What are your attitudes towards the law and towards the changes since the law enacted? 


\section{Appendix 8.2}

\section{INTERVIEW QUESTIONS \\ -- Officials from the Ministry of Agriculture and the Industry and Commerce Bureau}

The interview questions for Officials from the Ministry of Agriculture:

1. What is the background of the law?

2. How was the law initiated?

3. What are the anticipated effects of the law?

4. How do you assess the success of the law so far?

5. What are the major barriers to its success?

6. Have you had consideration that the law may have effects on indigenous knowledge practice?

7. What are your attitudes towards these effects?

Questions for Officials from the Industry and Commerce Bureau

1. What are your responsibilities to implement the law?

2. Is there any special supporting measure for implementing the law?

3. If yes, what are they?

4. How do you carry out these measures?

5. How many farmers' cooperative organisations have formed so far?

6. What are the anticipated effects of the law?

7. How do you assess the success of the law?

8. What are the main barriers to its implementation? 


\section{Appendix 8.3}

\section{INTERVIEW QUESTIONS}

-- Experts in Mongolian traditions, grassland conservation and community-based conservation and development

The interview questions with experts also tended to be broad. The broad questions created a space for the expert interviewees to lead the conversation and present more of their expertise.

Questions included:

1. What is your knowledge and experience with Mongolian traditional natural resource management?

2. What is your knowledge and experience with Mongolian traditional institutions?

3. What is your knowledge and experience with Mongolian traditional culture?

4. What is your knowledge and experience about the changes in these factors over the last 50 years?

5. What is your knowledge and experience about governmental policies and their effects on these changes?

6. What is your knowledge and experience with community cooperative activities? 


\section{Appendix 9.1}

A Summary of TEK Threats, Conservation Actions and Recommendations for Further Conservation Actions in Two Inner Mongolia Case Studies

\begin{tabular}{|c|c|c|}
\hline & $\begin{array}{c}\text { Bayin Case Study } \\
\text { (Chapter } 5 \text { in this thesis) }\end{array}$ & $\begin{array}{c}\text { Hatu Case Study } \\
\text { (Tang, 2007; Tang and Gavin, } \\
\text { 2010) }\end{array}$ \\
\hline \multirow[t]{2}{*}{ Direct threats } & $\begin{array}{l}\text { 1. Change of traditional } \\
\text { livelihood practices } \\
\text { 2. Loss of traditional rights } \\
\text { 3. Loss of traditional } \\
\text { institutions } \\
\text { 4. Change of environment } \\
\text { and natural resources } \\
\text { 5. Loss of pathways of TEK } \\
\text { transmission }\end{array}$ & $\begin{array}{l}\text { 1. Change of traditional } \\
\text { livelihood practices } \\
\text { 2. Loss of traditional rights } \\
\text { 3. Loss of traditional } \\
\text { institutions } \\
\text { 4. Change of environment and } \\
\text { natural resources } \\
\text { 5. Loss of pathways of TEK } \\
\text { transmission }\end{array}$ \\
\hline & & $\begin{array}{l}\text { 6. Loss of traditional religion } \\
\text { and belief }\end{array}$ \\
\hline \multirow[t]{2}{*}{ Underlying threats } & $\begin{array}{l}\text { 1. Government policy and } \\
\text { legislation } \\
\text { 2. Contact with other } \\
\text { cultural groups } \\
\text { 3. Economic development } \\
\text { pressure }\end{array}$ & $\begin{array}{l}\text { 1. Government policy and } \\
\text { legislation } \\
\text { 2. Contact with other cultural } \\
\text { groups } \\
\text { 3. Economic development } \\
\text { pressure }\end{array}$ \\
\hline & & $\begin{array}{l}\text { 4. Migration } \\
\text { 5. Relocation }\end{array}$ \\
\hline $\begin{array}{l}\text { TEK conservation } \\
\text { actions }\end{array}$ & $\begin{array}{l}\text { 1. Indigenous capacity } \\
\text { building } \\
\text { 2. Policy and legislation } \\
\text { 3. Community-based TEK } \\
\text { conservation activities }\end{array}$ & $\begin{array}{l}\text { 1. Indigenous capacity building } \\
\text { 2. Policy and legislation } \\
\text { 3. Community-based TEK } \\
\text { conservation activities }\end{array}$ \\
\hline \multirow[t]{2}{*}{$\begin{array}{l}\text { Recommendations } \\
\text { for further } \\
\text { conservation actions }\end{array}$} & $\begin{array}{l}\text { 1. Law and policy support } \\
\text { at a more comprehensive } \\
\text { level } \\
\text { 2. Community-based TEK } \\
\text { conservation activities } \\
\text { 3. Educational means }\end{array}$ & $\begin{array}{l}\text { 1. Law and policy support from } \\
\text { the authority at multiple levels } \\
\text { 2. Deepen community-based } \\
\text { TEK conservation activities } \\
\text { 3. Educational means }\end{array}$ \\
\hline & & $\begin{array}{l}\text { 4. Community-initiated TEK } \\
\text { research projects }\end{array}$ \\
\hline
\end{tabular}

The parts coloured in green are the common attributed shared by two case studies; the parts coloured in blue are the different attributes. 


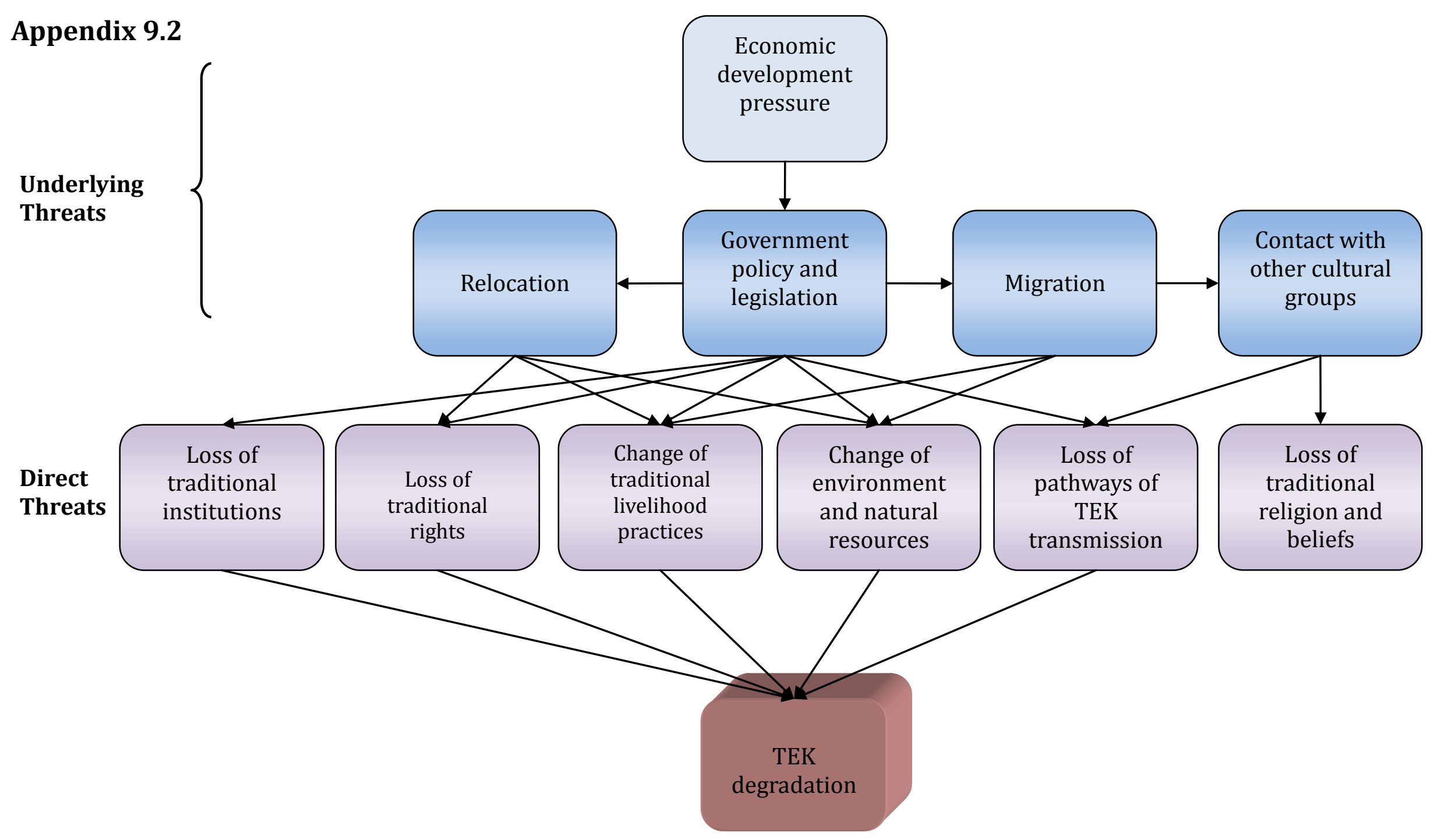

The causal web of TEK threats in Hatu gacha case study (western Inner Mongolia, China) 